\title{
Mechanisms of Dynamic Deformation and Failure in Ultra- High Molecular Weight Polyethylene Fiber-Polymer Matrix Composites
}

A Dissertation
Presented to
the faculty of the School of Engineering and Applied Science
University of Virginia
in partial fulfillment
of the requirements for the degree of
Doctor of Philosophy
Material Science and Engineering

Mark R. O’Masta

May 2014 


\section{APPROVAL SHEET}

The dissertation is submitted in partial fulfillment of the requirements for the degree of

Doctor of Philosophy

$\begin{array}{r}\text { Mark O'Masta } \\ \hline \text { Mark R. O’Masta, Author }\end{array}$

This dissertation has been read and approved by the examining committee:

Haydn Wadley

Haydn N.G. Wadley, Advisor

James Fitz-Gerald

James M. Fitz-Gerald, Committee Chair

Sean Agnew
Sean R. Agnew

Devin Harris

Devin K. Harris

Vikram Deshpande

Vikram S. Deshpande

Accepted for the School of Engineering and Applied Science:

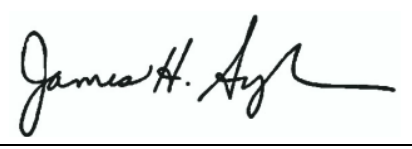

James H. Aylor, Dean

School of Engineering and Applied Science

May 2014 


\section{Abstract}

Ultra-high molecular weight polyethylene (UHMWPE) molecules, with molecular weights approaching $10^{7} \mathrm{Da}$ and lengths approaching $10 \mu \mathrm{m}$, can be gel spun and drawn into highly crystalline fibers with more than $95 \%$ of the molecules oriented in the fiber direction. The very high tensile strength (approaching $4 \mathrm{GPa}$ ) and elastic modulus (200 GPa) combined with a very low density $\left(970 \mathrm{~kg} \mathrm{~m}^{-3}\right)$ result in a fiber with very high specific strength and modulus. While the strength per unit mass of the materials in the fiber direction is $\sim 25$ times greater than that of conventional steels, weak (van der Waals) bonds between molecules leads to strengths transverse to the fibers of only a thousandth that in the fiber direction. This weak intermolecular strength also leads to creep deformation under prolonged loading at ambient temperatures, and complete failure of the polymer when the intermolecular bonds "melt" at $155^{\circ} \mathrm{C}$. These materials are therefore used in weight sensitive applications, where a high uniaxial stress must be supported for relatively short periods of time. Examples include mooring cables, the sails of racing ships and ballistic impact protection panels. For ballistic applications, the 10-20 $\mu \mathrm{m}$ diameter fibers are combined with compliant thermoplastic polymer matrices 
to form thin (typically $50 \mu \mathrm{m}$ thick) unidirectional plies containing $\sim 85 \%$ by weight fibers. These plies are then layered to form a cross-ply $\left(\left[0^{\circ} / 90^{\circ}\right]_{\mathrm{n}}\right)$ structure, and pressed (at $127^{\circ} \mathrm{C}$ ) to create a composite panel. This dissertation investigates the structure, mechanical properties and dynamic deformation and failure mechanisms during the ballistic impact of these UHMWPE reinforced $\left[0^{\circ} / 90^{\circ}\right]$ polymer matrix composites by a model projectile.

Six UHMWPE $\left[0^{\circ} / 90^{\circ}\right]$ polymer composite systems were investigated in the study. The laminates had measured tensile strengths (a fiber dominated property) in the range of $800-1100 \mathrm{MPa}$, which was 500-5,000 times higher than the laminates' measured interlaminar shear strengths (a matrix dominated property). Digital image correlation techniques have been used to show that the Poisson expansion of a ply under compressive loading was also highly anisotropic, with a Poisson's ratio of $v_{23}=0.5$ transverse to fiber direction, and $v_{13}=0$ in fiber direction. During uniform out of plane (through thickness) compressive loading of $\left[0^{\circ} / 90^{\circ}\right]$ composites, this anisotropic Poisson expansion of adjacent $90^{\circ}$ plies has been shown to cause fiber tension in the $0^{\circ}$ ply by a shear lag mechanism. Failure of the compressed sample occurs when the tension induced stress in the fibers reaches the plies failure strength (in excess of $1 \mathrm{GPa}$ ), and agreed well with experimental data collected on thick laminates with lateral dimensions substantially larger than the shear lag length.

The out of plane compressive strength of the $\left[0^{\circ} / 90^{\circ}\right]$ composites was discovered to be dependent upon the laminate thickness; as the laminate thickness was decreased the strength of the laminates decreased to $60 \%-70 \%$ of the indirect tension strength prediction. Using a combination of optical and ultrasonic C-scan imaging techniques in 
conjunction with micro-X-ray tomography, two classes of defects have been identified in the $\left[0^{\circ} / 90^{\circ}\right]$ composites. One defect type consisted of tunnel cracks that were parallel to the fibers in a ply and approximately equally spaced in the transverse direction. These are shown to form as a result of anisotropic thermal strains within the laminates during cooling after consolidation processing. The second void-like defect results from missing groups of fibers within each ply. Like the tunnel cracks, this defect extended many centimeters in a ply's fiber direction. While tunnel cracks were healed during ambient temperature out of plane compression, and therefore had little effect on a laminates out of plane compressive strength, the missing fiber defects significantly degraded the compressive strength of thin laminates. Compression tests using pressure sensitive film and acoustic emission monitoring reveal that regions containing missing fiber defects in thin laminates are shielded from load by defect free regions, which then fail at lower sample pressure during loading. A simple statistical model was developed that successfully predicted the contrast observed in optical and ultrasonic images, and the effect of missing fiber defects upon the out of plane compressive strength.

The dissertation also investigated the mechanisms of projectile penetration during impact of UHMWPE fiber-reinforced composites with a spherical projectile using model targets designed to dynamically load the laminates in different ways. The response of the samples were studied using a combination of synchronized high speed photography with three cameras, and 3D digital image correlation together with post-test characterization via X-ray tomography and optical microscopy. It was found that a rear supported laminate, which was prevented from deflecting, was progressively penetrated by the projectile. Since the projectile applied only a compressive pressure to the laminate, it is 
argued that penetration occurred by the indirect tension mechanism. Edge clamped laminates that are allowed to freely deflect have an improved impact resistance, especially if the projectile is fragmented before impacting the laminate, or the laminate is given an out of plane velocity prior to direct impact by the projectile. The results are used to propose a projectile penetration process model that incorporates both the activation of indirect tension and membrane stretching. It predicts that suppression of high compressive stress in the $\left[0^{\circ} / 90^{\circ}\right]$ laminate forces the laminate to respond in a bi-axial membrane stretching mode where the kinetic energy of the projectile is expended in the very significant work needed to stretch the laminate. This hypothesis was tested with a model impact target that spatially distributed the load to the laminate and was found to substantially increase the resistance of the laminate to penetration and failure. 


\section{Acknowledgements}

Haydn, your guidance and patience have made this journey tenable. You have taught me to be always inquisitive, always a visionary and never deterred. Thank you for your inspiration and enthusiasm and for your enduring encouragement of my (at times unconventional) research endeavors.

I would like to express my gratitude to each member of my committee, Sean Agnew, Vikram Desphande, James Fitz-Gerald and Devin Harris, for your continual advisement and thoughtful review of my work.

I would also like to express my utmost appreciation for all of my collaborators. I thank Vikram Deshpande, Julia Attwood, Kandan Karthikeyan and Ben Russell at the University of Cambridge for your numerous discussions and help in studying this research topic. I sincerely thank Frank Zok, Brett Compton and Nell Gamble at the University of California - Santa Barbara for your help with the ballistic experiments. I am also thankful to Dustin Crayton (UVA) and Jason Cain (Army Research Laboratory) for your help with the compression experiments. I have the sincerest gratitude for Harm 
van der Werff and Ulrich Heisserer at DSM for your knowledgeable insights, fruitful discussions and material support.

I would also like to recognize my laboratory mates and IPM staff: Kumar Dharmasena, Liang Dong, Tommy Eanes, David Glover, Rich Gregory, Ryan Holloman, Toni Kember, Adam Malcom, and Sherri Sullivan. I thank each of you for indispensible help over the years and for providing a richer, fuller and happier graduate experience.

I am forever grateful to my parents for their love, unwavering support and encouragement. Thank you for always pushing me to be a better person.

To my wife, Brenna, you have sacrificed more than anyone in this pursuit. I can never repay all that you have given, your love, affection, advice and support, and all of the sleep, weekends and time apart (both physically and mentally) that we have lost. I give you my deepest and most humble thank you.

This work was funded by the Office of Naval Research (ONR) under grant number N00014-07-1-0764 (Program manager, Dr. D. Shifler) and the Defense Advanced Research Projects Agency (DARPA) under grant number W91CRB-11-1-0005 (Program manager, Dr. J. Goldwasser). 


\section{Table of Contents}

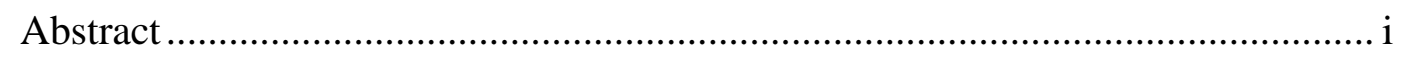

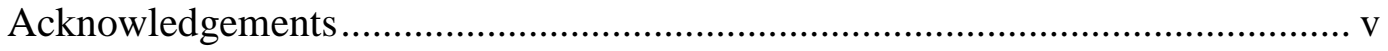

List of figures .............................................................................................

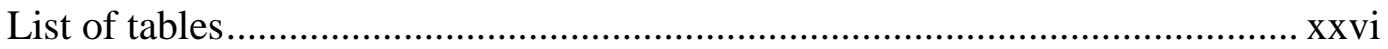

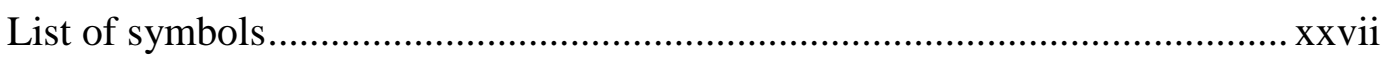

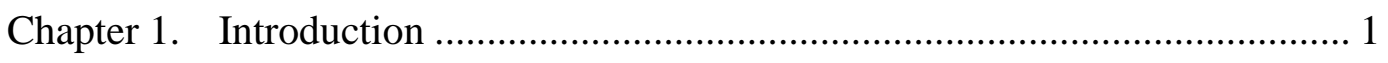

1.1. Impact resistant polyethylene ............................................... 1

1.2. Impact response of polymer matrix composites ............................. 6

1.3. Dissertation goals .............................................................. 12

1.4. Dissertation outline................................................................. 12

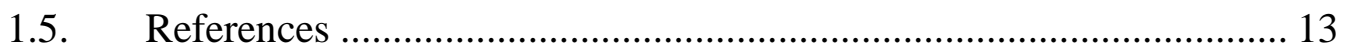

Chapter 2. UHMWPE fibers and composites........................................... 18

2.1. Processing, structure, and properties of UHMPWE fibers ................ 18 


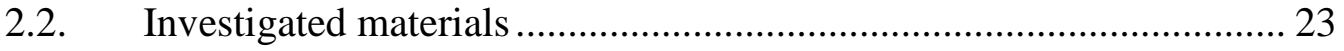

2.3. Laminate consolidation............................................................ 26

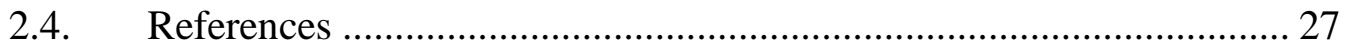

Chapter 3. Fiber and composite mechanical response ................................... 30

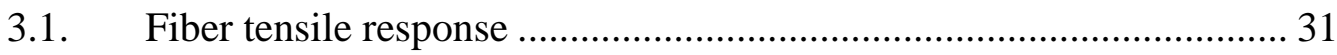

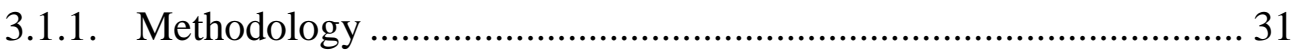

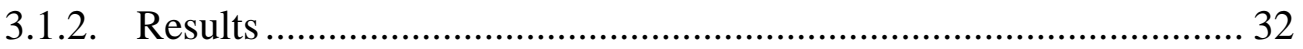

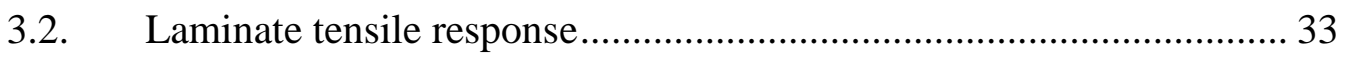

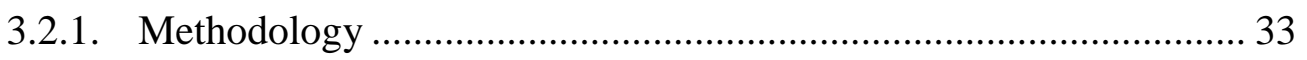

3.2.2. Results ................................................................................ 34

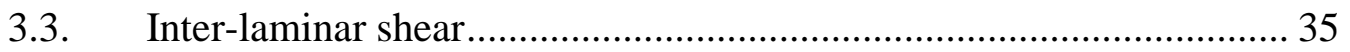

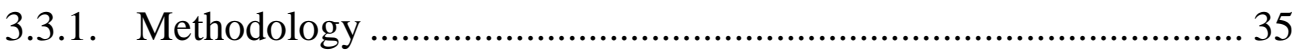

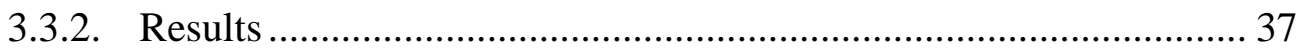

3.4. Shear strength dependence on compression ................................. 38

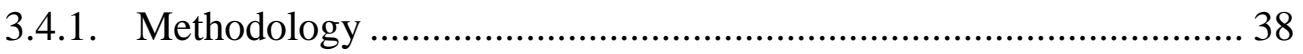

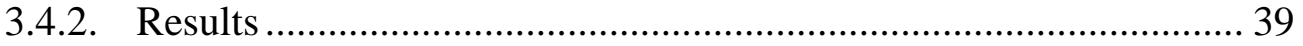

3.5. Compression of unidirectional laminates ..................................... 39

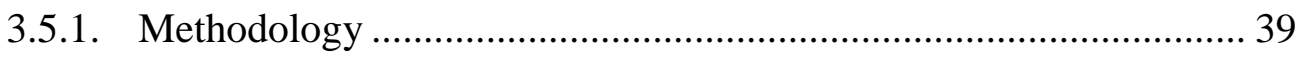

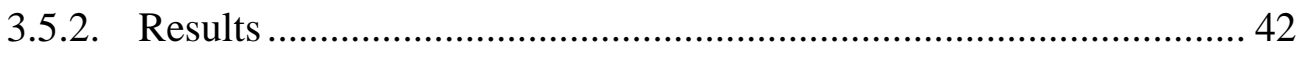

3.6. Conclusions ....................................................................... 45 


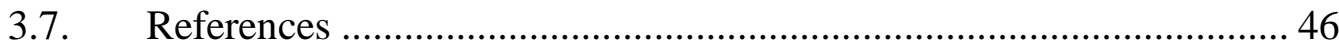

Chapter 4. Mechanisms of projectile penetration in HB26 encapsulated

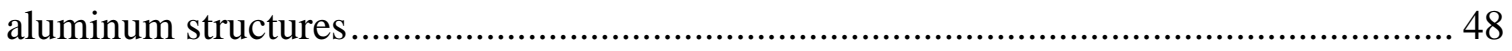

4.1. Materials and sample fabrication ............................................... 49

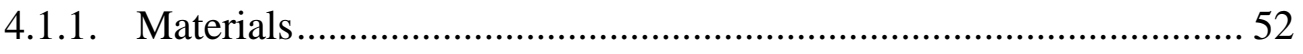

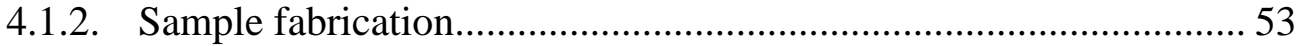

4.2. Impact test protocol ............................................................ 56

4.3. Impact response of targets ................................................... 57

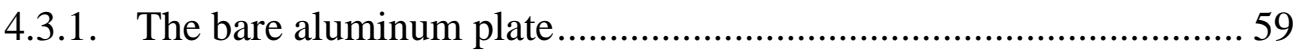

4.3.2. The front face cutout target ...................................................... 65

4.3.3. The baseline fully encased target ............................................... 69

4.3.4. The rear face cutout target...................................................... 75

4.4. Discussion of Dyneema penetration mechanisms ......................... 77

4.4.1. Dyneema plates resting on a strong foundation ........................... 79

4.4.2. Edge supported Dyneema ${ }^{\circledR}$ plates ............................................ 81

4.4.3. Residual velocity of the projectile/ejecta ..................................... 85

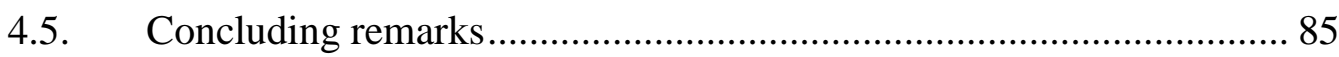

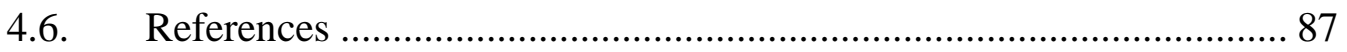

Chapter 5. Defect Dependent Transverse Compressive Strength of Ballistic

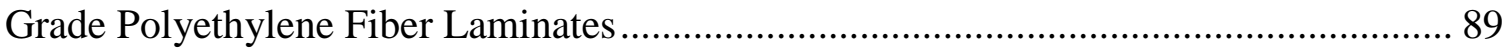


5.1. Materials and fabrication ....................................................... 93

5.1.1. Material Types..................................................................... 93

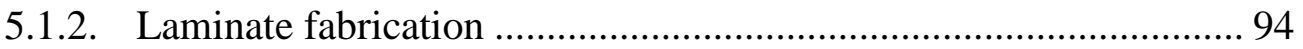

5.2. Review of the indirect tension model .......................................... 95

5.3. Transverse compressive strength ............................................. 97

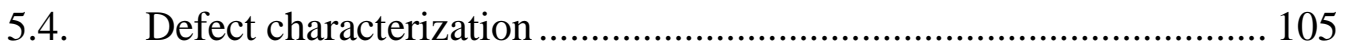

5.5. Pressure distribution, tunnel crack healing and ply thickness

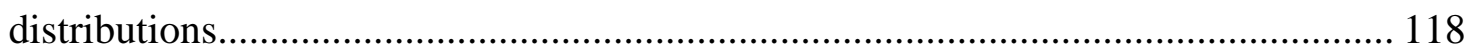

5.6. Modeling and simulation ..................................................... 124

5.7. Concluding remarks ........................................................... 136

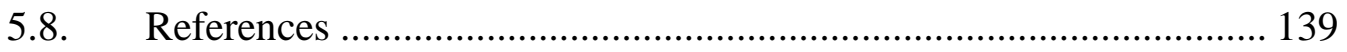

Chapter 6. Ballistic impact response of HB26 encased aluminum-alumina hybrid

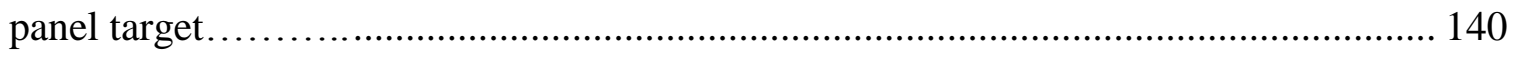

6.1. Sample fabrication ........................................................... 144

6.2. Impact tests and characterization........................................... 147

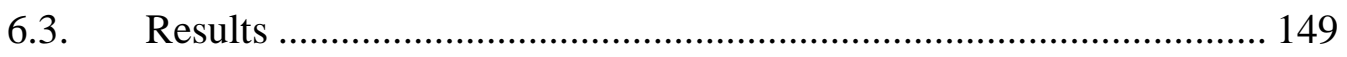

6.3.1. Encased hybrid targets - ceramic prism base impacts ................. 149

6.3.2. Encased hybrid targets - ceramic prism apex impacts................. 159

6.3.3. Rear face cutout targets - ceramic prism base impacts................ 160

6.3.4. Rear face cutout targets - ceramic prism apex impacts ................ 164 


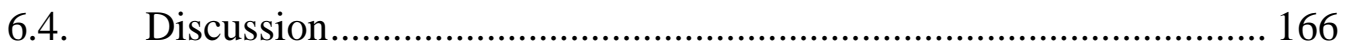

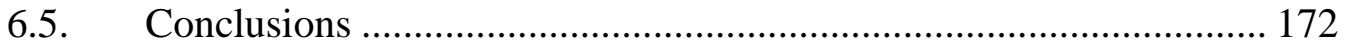

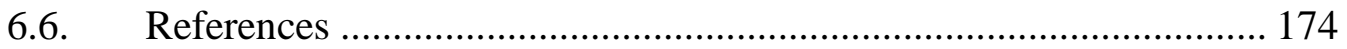

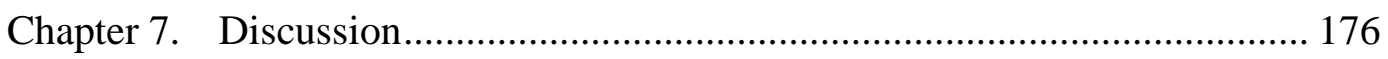

7.1. UHMWPE Composite Characterization...................................... 176

7.2. Impact Response Mechanisms............................................... 178

7.3. Laminate Defect Effects ........................................................... 185

7.4. Suggestions for future work ................................................. 186

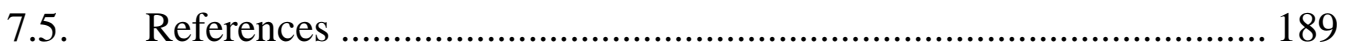

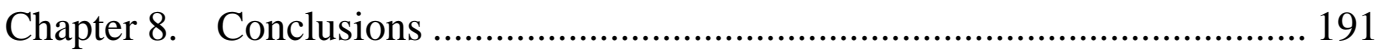

8.1. References ........................................................................ 197

Appendix A. Tensile properties of high-performance fibers ......................... 198

Appendix B. Measurement length changes from teh high speed images......... 203

Appendix C. X-ray tomogram analysis procedures .................................. 205 


\section{List of figures}

Figure 1.1. (a) Schematic illustration of the cross-section of a UHMWPE fiber (thermoplastic) polymer matrix $\left[0^{\circ} / 90^{\circ}\right]_{2}$ cross-ply tape used to form the laminate shown in (b) by out of plane compression at a pressure of 20.6 MPa and temperature of $127^{\circ} \mathrm{C}$

Figure 1.2. The effects of material properties on projectile penetration near the ballistic

limit of light targets.

Figure 1.3. Material property charts comparing the (a) tensile strength and Young's modulus and (b) specific toughness and extensional wave speed, $c_{L}=(E / \rho)^{1 / 2}$, of high performance fibers. Contours of the Cunniff [24] velocity, $\mathrm{c}^{*}$, are also plotted on (b)

Figure 1.4. (a) Schematic illustration of the deformation mechanisms occurring during the impact of a $\left[0^{\circ} / 90^{\circ}\right]_{3}$ fiber reinforced composite laminate. Magnified views that 
show (b) the wave propagation along the laminate and (c) the stress state under the projectile 8

Figure 1.5. (a) Schematic illustration of plies within a cross-ply laminate under a uniform compressive stress, $\sigma_{\mathrm{z}}$. Poisson lateral expansion in the fiber direction is much less than that transverse to the fibers. (b) Schematic illustrations of the stress within the composite predicted by the shear lag model. 11

Figure 2.1. (a) Packing of polyethylene molecules in an orthorhombic crystal structure. (b) Crystal texturing in an UHMWPE drawn fiber, where the molecules are oriented in the fiber orientation. This crystal structure can assemble as either (c) a foldedchain lamellar structure or (d) an extended-chain structure

Figure 2.2. The (a) gel-spinning process used to make UHMWPE fibers. The (b) gel is composed of folded-chain lamellar crystals that are spun (with minimal removal of solvent) into (c) a Xerogel of coplanar lamellar crystals. Red line represents the carbon chain C-C backbone. (d) Hot drawing removes the remained of the solvent and extends the lamellar chains into a chain-extended macro-conformation. 22

Figure 2.3. (a) XCT reconstruction of a consolidated HB26 laminate. (d) An SEM micrograph of a ply from a consolidated laminate cut normal to the filament direction. The $0^{\circ}$ filament ends were slightly smeared from the microtome blade. 24

Figure 3.1. A typical response of Dyneema ${ }^{\circledR}$ SK76 and X131 yarns when pulled in tension at a nominal strain rate of $10^{-2} \mathrm{~s}^{-1}$ 33

Figure 3.2. Cross-ply laminate sample design for measuring the tensile response. All dimensions are in millimeters. 34

Figure 3.3. The measured laminate tensile response for each grade of material. 35 
Figure 3.4. (a) Cross-ply laminate sample design for determining the interlaminar shear strength. (b) Magnified view of (a) identifying one of two shear planes. (c) Modified shear test where a out of plane compressive load is applied over the shear planes. 36

Figure 3.5. The inter-laminar shear stress, $\tau_{\mathrm{ZX}}$, plotted as a function of displacement in the X-direction for each material.

Figure 3.6. Load frame addition applies a compressive load, $\sigma_{\mathrm{z}}$, over the sample gauge section, $w L_{g}$, while the sample is pulled in tension. (a) shows an oblique view, while the view in (b) is normal to the $\mathrm{XZ}$ plane. 38

Figure 3.7. The influence of out of plane compression, $\sigma_{\mathrm{Z}}$, on (a) the inter-laminar shear stress, $\tau_{\mathrm{ZX}}$, plotted as a function of displacement and (b) the shear strength, $\tau$, for HB26.

Figure 3.8. Schematic illustration of an L square unidirectional sample under uniform out of plane compression. 40

Figure 3.9. The field of views as seen from (a) the left and (b) the right cameras of the speckle coated $\mathrm{YZ}$ plane of an $\mathrm{L}=10 \mathrm{~mm},\left[0^{\circ}\right]_{45}$, unidirectional HB26 sample.... 41

Figure 3.10. (a) The measured nominal stress versus strain of $\left[0^{\circ}\right]_{45}$, HB26 compression samples. (b) An image sequence showing the deformation of the $\mathrm{L}=13 \mathrm{~mm}$ sample. The view is normal to the $\mathrm{YZ}$ plane. Images of the $\mathrm{L}=13 \mathrm{~mm}$ sample, normal to the XY plane, are shown (c) before and (d) after testing. 43

Figure 3.11. The compressive load-unload response of an $\mathrm{L}=10 \mathrm{~mm},\left[0^{\circ}\right]_{45}, \mathrm{HB} 26$ laminate in the regime prior to shear failure. Plotted as functions of the compressive 
strain, $\varepsilon_{\mathrm{Z}}$, are (a) the compressive stress, $\sigma_{\mathrm{Z}}$, and (b) the transverse strains, $\varepsilon_{\mathrm{Y}}$ and $\varepsilon_{X}$

Figure 4.1. Sketches of the four model targets employed in this study. (a) The baseline target with the Al plate fully encased by Dyneema ${ }^{\circledR},(b)$ the bare Al plate, (c) the front face cutout target and (d) the rear face cutout target. All dimensions are shown in $\mathrm{mm}$.

Figure 4.2. A typical true stress - strain uniaxial tensile of the 6061-T6 aluminum target material.

Figure 4.3. A typical true stress - strain response when compressing a right circular cylinder cut from the 52100 spherical projectile. 53

Figure 4.4. Fabrication of the Dyneema ${ }^{\circledR}$ encased aluminum panels using two strips of HB26 Dyneema ${ }^{\circledR}$ pre-preg tape. All dimensions are given in $\mathrm{mm}$. 54

Figure 4.5. Schematic illustration of (a) the test fixture used to grip the targets and (b) the dynamic testing facility at Chesapeake Testing (Belcamp, MD) used to perform the ballistic experiments. 56

Figure 4.6. Summary of the measured residual (exit) velocity $\mathrm{V}_{\mathrm{r}}$ of the projectile/ejecta as a function of impact velocity $\mathrm{V}_{\mathrm{i}}$ for the four model targets. 59

Figure 4.7. The measured depth of penetration and crater diameter as a function of impact velocity for impacts against a $63.5 \mathrm{~mm}$ thick $\mathrm{Al}$ 6061-T6 plate...... 61

Figure 4.8. Photographs of $31.6 \mathrm{~mm}$ thick bare aluminum plates impacted at (a) $\mathrm{V}_{\mathrm{i}}=$ $1090 \mathrm{~m} \mathrm{~s}^{-1}$, (b) $\mathrm{V}_{\mathrm{i}}=1360 \mathrm{~ms}^{-1}$, (c) $\mathrm{V}_{\mathrm{i}}=1530 \mathrm{~ms}^{-1}$ and (d) $\mathrm{V}_{\mathrm{i}}=1647 \mathrm{~ms}^{-1}$ and sectioned along a central plane. 
Figure 4.9. The measured crater diameter in the Al plates of the different targets as a function of impact velocity for the four model targets. The state of the projectile is indicated in each case.

Figure 4.10. High speed image sequences showing the deformation of the bare $31.6 \mathrm{~mm}$ thick Al target impacted at (a) $\mathrm{V}_{\mathrm{i}}=1300 \mathrm{~m} \mathrm{~s}^{-1}$ and (b) $\mathrm{V}_{\mathrm{i}}=1610 \mathrm{~m} \mathrm{~s}^{-1}$. The images in (a) are of a profile view of the rear of the target while (b) shows an oblique view of the rear surface. Time $t=0$ corresponds to the instant that the projectile impacts the front face. 64

Figure 4.11. (a) A spatial reference for interpretation of the X-ray images shown in (b) and (c) of the rear Dyneema ${ }^{\circledR}$ laminate from a front face cutout target impacted at $V_{i}=1430 \mathrm{~m} \mathrm{~s}^{-1}$. The white areas and bright streaks are beam hardening artifacts.. 66

Figure 4.12. Synchronized high speed image sequences of the front face cutout target impacted at $V_{i}=1430 \mathrm{~m} \mathrm{~s}^{-1}$. Three different views are shown: (a) a profile view, and oblique views of the (b) rear and (c) front surfaces. A $12.7 \mathrm{~mm}$ spaced grid pattern is drawn on the Dyneema ${ }^{\circledR}$. The rectangular box in shown in (c) to indicate the position of the cutout section from the front face. Time $t^{\prime}=0$ corresponds to the instant that the deformation of the rear Dyneema ${ }^{\circledR}$ face initiates. 68

Figure 4.13. Photographs of the baseline fully encased target impacted at (a) $V_{i}=800 \mathrm{~m}$ $\mathrm{s}^{-1}$, (b) $\mathrm{V}_{\mathrm{i}}=1220 \mathrm{~m} \mathrm{~s}^{-1}$ and (c) $\mathrm{V}_{\mathrm{i}}=1360 \mathrm{~m} \mathrm{~s}^{-1}$ and sectioned along a central plane. A magnified view of the region around the arrested projectile from (c) is shown in (d). The images have been annotated to highlight salient features. 70

Figure 4.14. (a) The measured transverse deflection $\delta$ of the mid-span of the Dyneema ${ }^{\circledR}$ rear face, (b) the pull-in length, $\ell$, and (c) deformed rear laminate length $L$ of the 
baseline fully encased target as function of time $t^{\prime}$ for three select values of the impact velocity. The original length $\mathrm{L}_{0}$ of the undeformed laminate is indicated in (b), and time $\mathrm{t}^{\prime}=0$ corresponds to the instant that the deformation of the rear Dyneema ${ }^{\circledR}$ face initiates

Figure 4.15. Schematic showing (a) the pull-in of the Dyneema ${ }^{\circledR}$ encasing around the Al plate which results in large deflections of the rear Dyneema ${ }^{\circledR}$ face and (b) the definition of the pull-in length $\ell$ and deformed rear laminate length $\mathrm{L}$ as measured from the fiducial markers on the rear Dyneema ${ }^{\circledR}$ face. 74

Figure 4.16. High speed images showing an oblique view of the deformation of the rear surface of the rear face cut out specimen impacted at $V_{i}=1420 \mathrm{~m} \mathrm{~s}^{-1}$. A $12.7 \mathrm{~mm}$ spaced grid pattern is drawn on the Dyneema ${ }^{\circledR}$. Time $t=0$ corresponds to the instant that the projectile impacts the target.

Figure 4.17. Schematic of the setup used by Karthikeyan et al. [2] to measure the ballistic performance of HB26 Dyneema ${ }^{\circledR}$ plates impacted by a $12.7 \mathrm{~mm}$ diameter steel sphere.

Figure 4.18. The measured (a) projectile/ejecta residual velocity (circles $-0-$ ) and (b) fraction of perforated plies (squares $-\square-$ ) as a function of the velocity impacting the laminate on the front and rear sides of the targets. In (b) we include data of a similar thickness edge clamped HB26 plate data from [2]. 78

Figure 4.19. (a) Schematic showing the sequence of events for the perforation of the Dyneema ${ }^{\circledR}$ on the front face of the model targets. (b) Schematic illustrating the pull-in of the Dyneema ${ }^{\circledR}$ as the projectile impacts the rear Dyneema ${ }^{\circledR}$ face of the model targets. Magnified views of this impact event by either an intact or 
fragmented projectile are included. (c) A photograph of the central section through the fully encased specimen impacted at $\mathrm{V}_{\mathrm{i}}=110 \mathrm{~m} \mathrm{~s}^{-1}$ showing the partial perforation of the Dyneema ${ }^{\circledR}$ front laminate 80

Figure 4.20. (a) A schematic illustrating the penetration of the projectile through the fully encased target showing the bulging of the rear surface of the Al plate which accelerates the Dyneema ${ }^{\circledR}$ rear face prior to impact by the projectile. (b) A schematic illustrating the deflection, $\delta_{\text {peak }}$, of the rear Dyneema ${ }^{\circledR}$ face as a function of time $t^{\prime}$. The point at which the projectile impacts the Dyneema ${ }^{\circledR}$ is indicated (red circles) and the slope of the $\delta_{\text {peak }}$ versus $\mathrm{t}^{\prime}$ at that instant is denoted by $\delta D$. 84

Figure 5.1. (a) Schematic illustration of an L square by $\mathrm{H}$ thick sample under uniform compression. Compressive and resultant tensile stresses scale with the red color shading. (b) Schematic illustration of plies within a cross-ply laminate under a uniform compressive stress, $\sigma_{\mathrm{z}}$. Poisson lateral expansion in the fiber direction is much less than that transverse to the fibers. (c) Schematic illustrations of the stress within the composite predicted by the shear lag model. 90

Figure 5.2. High-speed image sequence of the failure of a $\left[0^{\circ} / 90^{\circ}\right]_{80}$ laminate loaded in out of plane uniform compression. An exposure time $0.83 \mu$ s was used.

Figure 5.3.Contour plots of the pressure between a $10 \mathrm{~mm}$ thick x $30 \mathrm{~mm}$ x $30 \mathrm{~mm}$ X131 sample and the loading platen after application of a nominal compressive stress of (a) $100 \mathrm{MPa}$ and (b) $200 \mathrm{MPa}$. The length of the shear lag region is marked. For brevity, only a $15 \mathrm{~mm}$ corner section of the specimen is shown.

Figure 5.4. Transverse compression strength, $\sigma_{\max }$, plotted as a function of sample length, $\mathrm{L}$, for all laminate grades. Scatter is seen in the measured strength as well as 
significant deviation from predicted values for thinner laminates at large coupon sizes. 100

Figure 5.5. The nominal transverse compressive stress, $\sigma_{\mathrm{n}}$, and the amplitude of acoustic emission (AE) events are plotted against nominal strain, $\varepsilon_{\mathrm{n}}$, for three $\mathrm{L}=12 \mathrm{~mm}$ $\left[0^{\circ} / 90^{\circ}\right]_{8}$ HB26 samples. (b) The compressive strength, $\sigma_{\max }$, of $\left[0^{\circ} / 90^{\circ}\right]_{8} \mathrm{HB} 26$ samples are plotted as a function of the number of AE events. 103

Figure 5.6. Plots of (a) the total AE events prior to final failure and (b) large AE events $\geq 110 \mathrm{~dB}$ prior to final failure as functions of compressive strength, $\sigma_{\max }$, for $\mathrm{L}=20$ $\mathrm{mm}$, thick $\left[0^{\circ} / 90^{\circ}\right]_{120}$ laminates. 105

Figure 5.7. Optical backlit photographs of $\left[0^{\circ} / 90^{\circ}\right]_{8}$ laminated plates of UHMWPE fiber laminates. 107

Figure 5.8. Optical transmission photographs of $\left[0^{\circ} / 90^{\circ}\right]_{60}$ laminated plates. The manufacture's printed brand identifiers are marked. 108

Figure 5.9. Ultrasonic transmission amplitude contour maps of the same $\left[0^{\circ} / 90^{\circ}\right]_{8}$ UHMWPE laminates shown in Figure 5.7. 110

Figure 5.10. MicroXCT orthogonal cross-sections of a $\left[0^{\circ} / 90^{\circ}\right]_{8} \mathrm{HB} 26$ laminate through a region of low optical attenuation (a-c) and a region of high attenuation (d-f)... 112

Figure 5.11. Histograms of the total void thickness and laminate thickness for regions of low optical attenuation (in Figure 5.10) are shown in (a) and (b). (c) and (d) show analogous data for high attenuation regions. 115

Figure 5.12. (a) Backlit optical transmission image of an unconsolidated HB26 tape. (b) A radiograph of the region highlighted in part a. (c-f) Reconstructed $\mu \mathrm{XCT}$ orthogonal cross-sections of the region highlighted in part a. 
Figure 5.13. (a) Backlit photograph of a pristine $\mathrm{L}=30 \mathrm{~mm}$, thin $\left[0^{\circ} / 90^{\circ}\right]_{8} \mathrm{HB} 26$ laminate test sample. Pressure sensitive film read after compressive loading to a nominal stress of (b) $\sigma_{\mathrm{n}}=100 \mathrm{MPa}$ and (c) $\sigma_{\mathrm{n}}=300 \mathrm{MPa}$. Simulations of the backlit image (c) and pressure maps (e and f) are shown along the bottom row... 119

Figure 5.14. (a) Backlit photograph of a pristine $\mathrm{L}=30 \mathrm{~mm}$, thick $\left[0^{\circ} / 90^{\circ}\right]_{120} \mathrm{HB} 26$ laminate test sample. Pressure sensitive film readings from uniform compressive loading to a nominal stress of (b) $\sigma_{\mathrm{n}}=100 \mathrm{MPa}$ and (c) $\sigma_{\mathrm{n}}=300 \mathrm{MPa}$. Simulations of the backlit image (c) and pressure maps (e and f) are shown along the bottom row.

Figure 5.15. Two $\mu \mathrm{XCT}$ cross-sections of a single location within a $\left[0^{\circ} / 90^{\circ}\right]_{8} \mathrm{HB} 26$ sample: (a) In its pristine state and (b) after compressing to $400 \mathrm{MPa}$ and unloading. Measurements reveal variability in both inter- and intra-ply thickness 122

Figure 5.16. The compressive strength, $\sigma_{\max }$, as a function of sample length, L, of laminates of varying ply thickness, h, made from a unidirectional HB26 pre-preg ply.

Figure 5.17. (a) Schematic illustration showing how sets of missing fibers within a ply lead to a laminate with fiber aligned void-like defects. (b) Definition of the defect ply fraction, $\eta$. (c) A $\left[0^{\circ} / 90^{\circ}\right]_{4}$ laminate with defect voids. (d) Hypothetical rearrangement of voids where the thickness of overlapping voids is summed and the laminate area is then divided into areas factions, $\mathrm{P}$, having the same overlapping void thickness. Compression of the laminate to a nominal strain, $\varepsilon_{\mathrm{n}}$, will then lead to incremental loading of laminate area. 126 
Figure 5.18. Simulations of a $\left[0^{\circ} / 90^{\circ}\right]_{8}$ laminate with defects assuming a plate width to void width ratio of 150 and a void probability of (a) $p=0.05$, (b) $p=0.10$ and (c) $p$ $=0.15$. Additional simulations using $\mathrm{p}=0.10$ for lay-ups of increasing thickness are shown in (d) $\left[0^{\circ} / 90^{\circ}\right]_{30},(\mathrm{e})\left[0^{\circ} / 90^{\circ}\right]_{60}$ and (f) $\left[0^{\circ} / 90^{\circ}\right]_{120}$

Figure 5.19. Probability mass $\mathrm{P}$ of the local fraction of defective plies d. Results are shown in (a) for a 16 ply laminate with various defect probabilities $\mathrm{p}$ and in (b) for the number of plies in a laminate assuming $\mathrm{p}=0.10$.

Figure 5.20. Predicted nominal stress, $\sigma_{\mathrm{n}}$, normalized by the ideal plateau strength, $\sigma^{\infty}{ }_{\mathrm{i}}$, and plotted as a function of strain, $\varepsilon_{\mathrm{n}}$, for HB26 laminates of infinite in-plane dimension $(L \rightarrow \infty)$, given $\eta=0.4$

Figure 5.21. Predicted strength as a function of the number of laminated plies, N, for an HB26 laminate of infinite in-plane dimension $(L \rightarrow \infty)$. (a) Assumes $\eta=0.4$ and investigates the influence of defect probability, p, while (b) shows the effect of defect to ply thickness fraction, $\eta$, for $p=0.10$. Also plotted as a function of the number of laminated plies in (a) is the normalized average strength and standard deviation for HB26 samples for $\mathrm{L}=20 \mathrm{~mm}$ to $30 \mathrm{~mm}$.

Figure 6.1. Schematic illustration of a corrugated aluminum sandwich structure with an alumina filled (hybrid) core after impact by a hardened steel sphere. The length of the out of plane displaced region on the rear face depended upon impact location. (a) A ceramic prism base impact resulted in a region of displacement $2 \mathrm{~L}_{c}$ wide, while (b) shows that an impact at the apex of a prism led to a displacement of half this width. 143 
Figure 6.2. Schematic illustrations of the two target types investigated in this study. (a) Shows the fully Dyneema ${ }^{\circledR}$ laminate encased sample design with dimensions defined in (b). The sample design with a part of the back laminate removed is shown in (c) together with a coordinate system. Reference planes for subsequent analysis are also shown in (a).

Figure 6.3. The fabrication sequence for making the Dyneema HB26 encased hybrid core panels. 146

Figure 6.4. Schematic illustration of the ballistic test fixture and sample support configuration. . 148

Figure 6.5. Schematic illustrations showing (a) the top and (b) the rear perspective views of the impact test geometry used to record 3D DIC measurements. 149

Figure 6.6. Transverse cross-sections of Dyneema ${ }^{\circledR}$ encased hybrid targets impacted by the $12.7 \mathrm{~mm}$ diameter hardened steel sphere on a prism base. Wavy vertical lines are an artifact of the water-jet sectioning process

Figure 6.7. XCT reconstructions of longitudinal (a-d and g-i) and in-plane (e, f, j and k) cross-sectional renderings of encased hybrid targets impacted at $\mathrm{V}_{\mathrm{i}}=1.37 \mathrm{~km} \mathrm{~s}^{-1}$ (a$\mathrm{f})$, and $\mathrm{V}_{\mathrm{i}}=2.26 \mathrm{~km} \mathrm{~s}^{-1}(\mathrm{~g}-\mathrm{k})$. The arrow in (a) indicates impact direction for both samples. 155

Figure 6.8. Transverse cross-sections of encased hybrid targets impacted at either (a) a prism base or (b) a prism apex. Prominent laminate failure mechanisms are identified. (c) Magnified view of the perforated region of the laminate shown in (b). 
Figure 6.9. High-speed video image sequence of an encased hybrid target during prism base impact at $\mathrm{V}_{\mathrm{i}}=2.70 \mathrm{~km} \mathrm{~s}^{-1}$. 159

Figure 6.10. (a) High-speed video image sequence showing an oblique rear view of the back aluminum surface of a rear cutout target that was prism base impacted at $\mathrm{V}_{\mathrm{i}}=$ $1.71 \mathrm{~km} \mathrm{~s}^{-1}$. A map of the out of plane surface velocity, $\delta$, determined from DIC measurements is superimposed. (b) High speed video images showing the out of plane deflection during sample impact.

Figure 6.11. The out of plane deflection history along (a) transverse and (b) longitudinal profile coordinates, as identified in Figure 6.10(a), for the rear face cutout target prism base impacted at $\mathrm{V}_{\mathrm{i}}=1.71 \mathrm{~km} \mathrm{~s}^{-1}$. Analogous data for the sample impacted at $\mathrm{V}_{\mathrm{i}}=2.29 \mathrm{~km} \mathrm{~s}^{-1}$ is shown in (c) and (d). 162

Figure 6.12. The peak out of plane velocity, $\delta p e a k$, versus time for rear face cutout targets. Two of the samples were impacted at a prism base and the third at a prism node. The terminal residual velocities after perforation are also shown. 163

Figure 6.13. The debris residual velocity, $\mathrm{V}_{\mathrm{r}}$, and maximum out of plane velocity, $\delta$ max, of rear face cutout targets versus impact velocity, $\mathrm{V}_{\mathrm{i}}$. Data for prism base impact is shown in dark $(\delta \max )$ and light $\left(\mathrm{V}_{\mathrm{r}}\right)$ blue. Data for a prism apex impact is shown in red $(\delta \max )$ and pink $\left(\mathrm{V}_{\mathrm{r}}\right)$.

Figure 6.14. High-speed video image sequence of the aluminum surface of a rear face cutout target impacted at $\mathrm{V}_{\mathrm{i}}=2.00 \mathrm{~km} \mathrm{~s}^{-1}$ on a prism apex. A map of the DIC generated out of plane velocity, $\delta$, component is superimposed. 165

Figure 6.15. The temporal evolution of the deflection profile of a rear face cutout target impacted at a prism apex with a velocity of $V_{i}=2.00 \mathrm{~km} \mathrm{~s}^{-1}$ 166 
Figure 6.16. Schematic illustration showing the loading sequence of the rear laminate of the encased hybrid target impacted on a prism base. The rear laminate is (a) initially accelerated by the bulging rear face sheet and (b) subsequently impacted by the released debris fragments. (c) Proposed defection history of the encased hybrid and encased aluminum targets both before and after impact by the debris/projectile. . 169

Figure 7.1. Three distinct loading scenarios investigated in the encased aluminum study used to elucidate penetration mechanisms. (a) An intact projectile impacting a foundation supported laminate. (b) An intact projectile impacting an edge-clamped laminate. (c) A fragmented projectile impacting an edge-clamped laminate. 179

Figure 7.2. Schematic illustrations of an encased aluminum target after impact by a steel sphere. (a) Plate bulging prior to perforation pre-accelerates the edge-clamped rear laminate. The depth of penetration in the laminate upon impact by a projectile that penetrated the plate then depended upon whether the projectile (b) remained intact or (c) had fragmented. 181

Figure 7.3. A proposed sequence of events for when (a) a spherical projectile impacts a stationary $\left[0^{\circ} / 90^{\circ}\right]$ polymer composite. (b) Inertial resistance prevents the laminate from immediately deflecting upon impact. The laminate underneath the projectile is placed in compression. This pressure is converted into a tensile stress in a ply's fiber direction by anisotropic expansion of adjacent $0^{\circ} / 90^{\circ}$ plies.(c) The velocity of the projectile is reduced by the work done in progressively failing the plies. If sufficient time has elapsed, the laminate may be simultaneously under membrane stretching and indirect tension. (d) If the laminate is sufficiently resistant to the 
penetration forces, it may enter into large deflections and resistant forces to stretching of the laminate will bring the projectile to rest.

Figure 7.4. Average compression strength, $\sigma_{\max }$, plotted as a function of sample length, L, for all UHMWPE fiber laminate grades and two UHMWPE solid-state laminate grades, BT10 Dyneema ${ }^{\circledR}$ and HSBD20A Tensylon ${ }^{\circledR}$ (DuPont, USA). BT10 data is from private communication with J.P. Attwood [17]. 187

Figure B.1. Sketch showing the oblique visualization of the grid on the Dyneema ${ }^{\circledR}$ encasing and the transformation used to correct for the parallax error. 


\section{List of tables}

Table 2.1. DSM reported yarn tensile properties [From private communications].......... 23

Table 2.2. Constituent materials, architecture and mechanical properties of the various

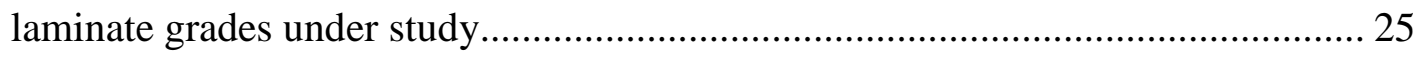

Table 5.1. Ply count to nominal thickness table for $\left[0^{\circ} / 90^{\circ}\right]$ n laminates. ...................... 95

Table 6.1. Impact results for the encased hybrid and rear face cutout targets ............... 151

Table 6.2. The ballistic limits of reference targets $\left(\rho_{\mathrm{a}}=97 \mathrm{~kg} \mathrm{~m}^{-2}\right)$ presented by the highest impact velocity that failed to completely perforate the target and the lowest

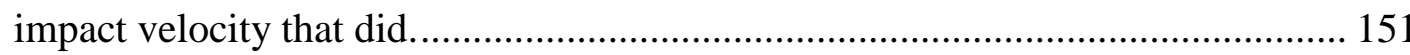

Table 6.3. The minimum impact velocity for penetration (first ply failure) and perforation (complete laminate failure) of $\sim 5.9 \mathrm{~mm}$ thick HB26 laminates when impacted by a

$12.7 \mathrm{~mm}$ diameter steel sphere.

Table A-4. The data for the mechanical properties high performance fibers used to generate Fig. 1. The sources of information are included in the table for each case. 


\section{List of symbols}

$a$

$c^{*}$

$c_{H}$

$c_{L}$

$d$

$h$

$h_{d}$

$\ell$

$\ell_{1}$

$\ell_{2}$

$\mathrm{n}$

$r$

$t$
A constant used in the indirect tension model

Cunniff index

Plastic hinge wave speed

Extensional wave speed

Ratio of defective plies to laminated plies (defective ply ratio)

Ply thickness

Thickness of a single defect

Total pull-in of laminate to the rear face of a ballistic target

Displacement of fiducial marker 1 on the rear face of a ballistic target

Displacement of fiducial marker 2 on the rear face of a ballistic target

Repeated $\left[0^{\circ} / 90^{\circ}\right]_{\mathrm{n}}$ lay-ups in laminate

Sum of overlapping plies with a defect

Fiber diameter 
w Inter-laminar shear strength sample width

$y_{s} \quad$ Shear lag length

$E_{f} \quad$ Longitudinal Young's modulus of ply

$E_{m} \quad$ Transverse Young's modulus of ply

$G_{12} \quad$ In-plane shear modulus

H Sample thickness

$L \quad$ In-plane sample length

$L_{0} \quad$ Original section length between two fiducial markers on the rear laminate of a ballistic target

$L_{i} \quad$ Arc length between two fiducial markers on the rear laminate of a ballistic target

$L_{g} \quad$ Inter-laminar shear strength sample gauge length

$V_{i} \quad$ Projectile velocity at target impact

$V_{i}^{*} \quad$ Impact velocity against the rear laminate of a target

$V_{r} \quad$ The Z-component residual (exit) velocity of the leading edge of ejecta

$N \quad$ Number of plies in laminate

$X, Y, Z \quad$ Global coordinate system

$\delta \quad$ Out of plane deflection

$\dot{\delta} \quad$ Rate of out of plane deflection

$\dot{\delta}_{\max } \quad$ Maximum out of plane velocity

$\dot{\delta}_{\text {peak }} \quad$ Out of plane velocity at location of peak deflection 
$\dot{\delta}_{D} \quad$ The out of plane velocity of the rear laminate just prior to impact by a projectile or debris

$\varepsilon_{f} \quad$ Strain to failure

$\varepsilon_{n} \quad$ Nominal compressive strain

$\mu \quad$ Shear strength pressure sensitivity coefficient

$\eta \quad$ Defect thickness to ply thickness fraction (defect ply thickness fraction)

$v \quad$ Poisson's ratio of ply

$\rho \quad$ Material density

$\sigma \quad$ Stress

$\sigma_{f} \quad$ Ply tensile strength

$\sigma_{\max } \quad$ Nominal compressive strength

$\sigma_{n} \quad$ Nominal compressive stress

$\sigma_{i}^{\infty} \quad$ Predicted compressive strength at $L \rightarrow \infty$

$\sigma_{\max }^{\infty} \quad$ Predicted defect compressive strength at $L \rightarrow \infty$

$\tau_{0} \quad$ Interlaminar shear strength under no compression

$\tau \quad$ Interlaminar shear strength under compression 


\section{Chapter 1. Introduction}

\subsection{Impact resistant polyethylene}

Ethylene is the simplest and most common organic monomer [1]. Polymerization of the ethylene monomer $\left(-\mathrm{CH}_{2}-\right)$ results in polyethylene $\left(-\left[\mathrm{CH}_{2}-\mathrm{CH}_{2}\right]_{\mathrm{n}}-\right)$. By the use of appropriate catalysts and synthesis conditions [2], it is possible to manipulate the molecular weight and molecular architecture of the polymer: both of which have been shown to govern its strength and modulus [3]. Low-density polyethylene (LDPE), which has a number-average molecular weight less than 100,000 Da, is extensively used for the making of films and thin sheet [2]. It is created by free-radical polymerization, which results in a high degree of chain branching with a mixture of short and long chains giving it flow characteristics useful for film production [2]. High-density polyethylene (HDPE) can be made using Zeigler-Natta catalysts that limit chain branching, and this molecule has a higher tensile strength than LDPE, and is commonly used in commercial products (e.g. milk containers and bottle caps). Through careful control of the polymerization process it is now possible to make ultrahigh molecular weight polyethylene (UHMWPE) 
with a molecular weight approaching $10^{7} \mathrm{Da}[2]$. By dispersing these giant molecules (whose lengths approach $10 \mu \mathrm{m}$ ) in appropriate solvents, patented gel spinning fabrication methods have been used to make UHMWPE fibers [2,4-6]. If the solvent is carefully removed after gel spinning, and the fibers are stretched under conditions that avoid fracture, it is possible to fabricate fibers in which $95 \%$ or more of the extended chain molecules crystallize into an orthorhombic structure with their c-axis aligned in the fiber direction [7-12]. Commercial fibers made in this way have a density of $970 \mathrm{~kg} \mathrm{~m}^{-3}$ and tensile strengths and moduli controlled by the backbone $\mathrm{sp}^{3}$ bonds that are greater than 3.5 and150 GPa, respectively, implying a specific strength 5-10 times that of steel. However, the weak (van der Waals) bonds between molecules leads to creep deformation when loaded at ambient temperatures $[13,14]$, and complete failure of the polymer when the intermolecular bond "melt" at $155^{\circ} \mathrm{C}$ [2]. These weak bonds also result in very poor in-plane shear modulus and strength as well as very low resistance to compressive loading in the fiber direction, which preclude its use for most structural applications. Typical applications of UHMWPE are therefore restricted to applications were the unidirectional tension loads are applied for short times or in applications where the temperatures are low. Examples include fishing lines and mooring cables. By creating $\left[0^{\circ} / 90^{\circ}\right]$ composite lay-ups, it also can be used in biaxial loading situations such as for the sails for racing ships, medical implants, protective gloves and ballistic impact protection panels [13].

Commercial materials for ballistic applications consist of high performance fibers, measuring 10-20 $\mu \mathrm{m}$ in diameter, that are combined with thermoplastic polymer matrices to form thin (typically $50 \mu \mathrm{m}$ thick) unidirectional plies containing $\sim 85 \%$ by volume 
fibers. Four of these plies are typically combined to form a cross-ply, $\left[0^{\circ} / 90^{\circ}\right]_{2}$, tape,

Figure 1.1(a). The tapes can be stacked and hot pressed to form cross-ply, $\left[0^{\circ} / 90^{\circ}\right]_{\mathrm{n}}$ laminates (Figure 1.1(b)) that are molded to form a protective structure [15] or attached to the rear of an armor system $[16,17]$.

(a) Cross-section of pre-preg tape

(b) Laminate lay-up of tapes

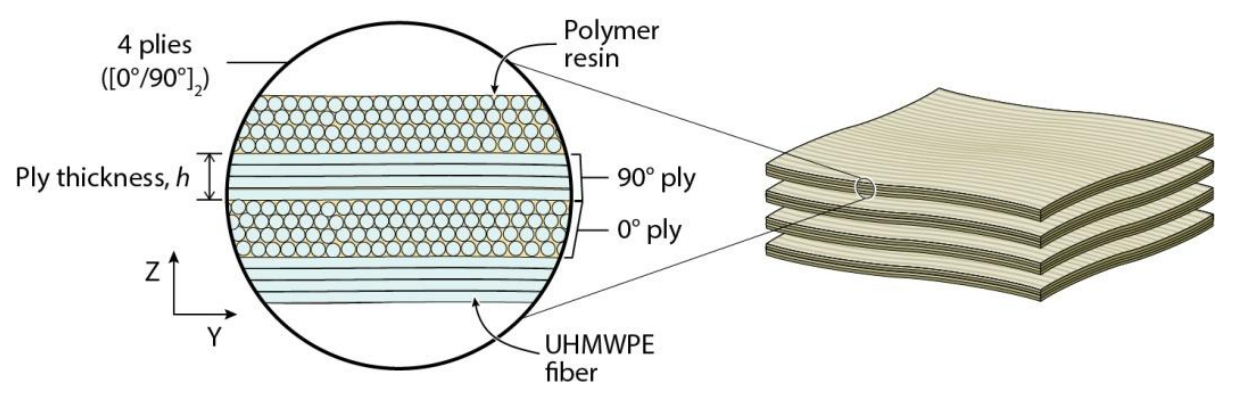

Figure 1.1. (a) Schematic illustration of the cross-section of a UHMWPE fiber (thermoplastic) polymer matrix $\left[0^{\circ} / 90^{\circ}\right]_{2}$ cross-ply tape used to form the laminate shown in (b) by out of plane compression at a pressure of $20.6 \mathrm{MPa}$ and temperature of $127^{\circ} \mathrm{C}$.

In the ballistic application, they are placed behind a hard (and ideally tough) material that deforms and/or fragments the projectile, Figure 1.2. This then disperses the load upon the ultra-high strength fiber reinforced laminate which then serves as a spall shield to catch debris. In a well-designed protection concept, synergies are sought between the two components of the armor system to maximize performance.

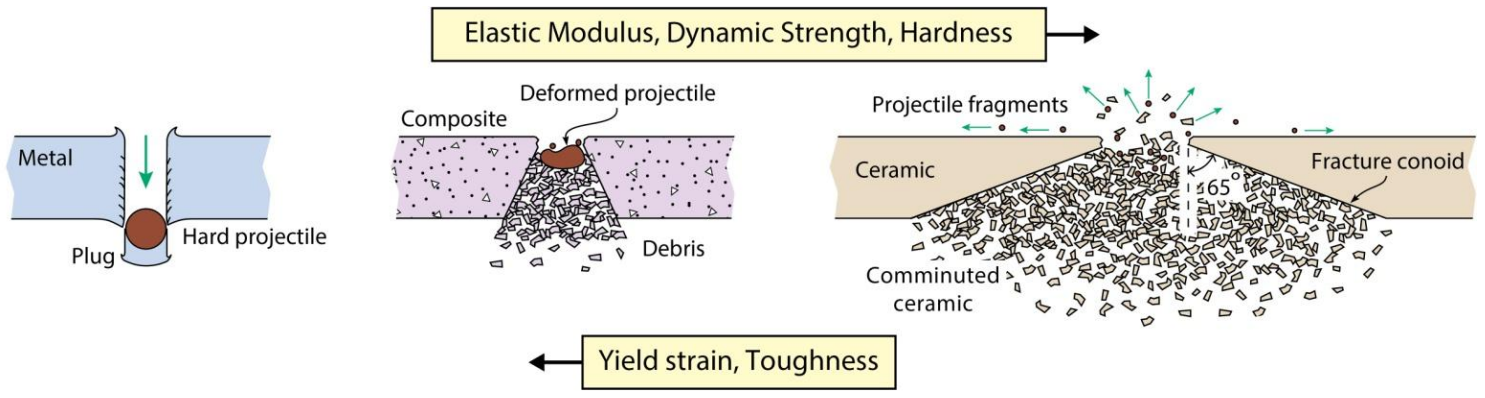

Figure 1.2. The effects of material properties on projectile penetration near the ballistic limit of light targets. 
Many studies [18-23] of the transverse impact of fibers suggest that to maximize performance, the fibers intended for use in spall shields need to possess a balance of properties including: (i) a high tensile strength to resist the significant stretching forces during (end restrained) fiber deflection, (ii) a high strain-to-failure to convert debris kinetic energy in to stored potential (strain) energy, (iii) a high elastic modulus to reduce dynamic out-of-plane (transverse) displacements and (iv) a low density if intended for mobile applications. Many fibers have been (or are being) developed for ballistic applications, and Figure 1.3(a) plots the quasi-static tensile strength and Young's modulus of many of these materials ${ }^{1}$. However, the material property chart does not address the role of fiber density, which is critical in some applications, nor the influence of the failure strain, which governs the mechanical work that can be stored.

\footnotetext{
${ }^{1}$ The sources of the material data are tabulated in Appendix A.
} 

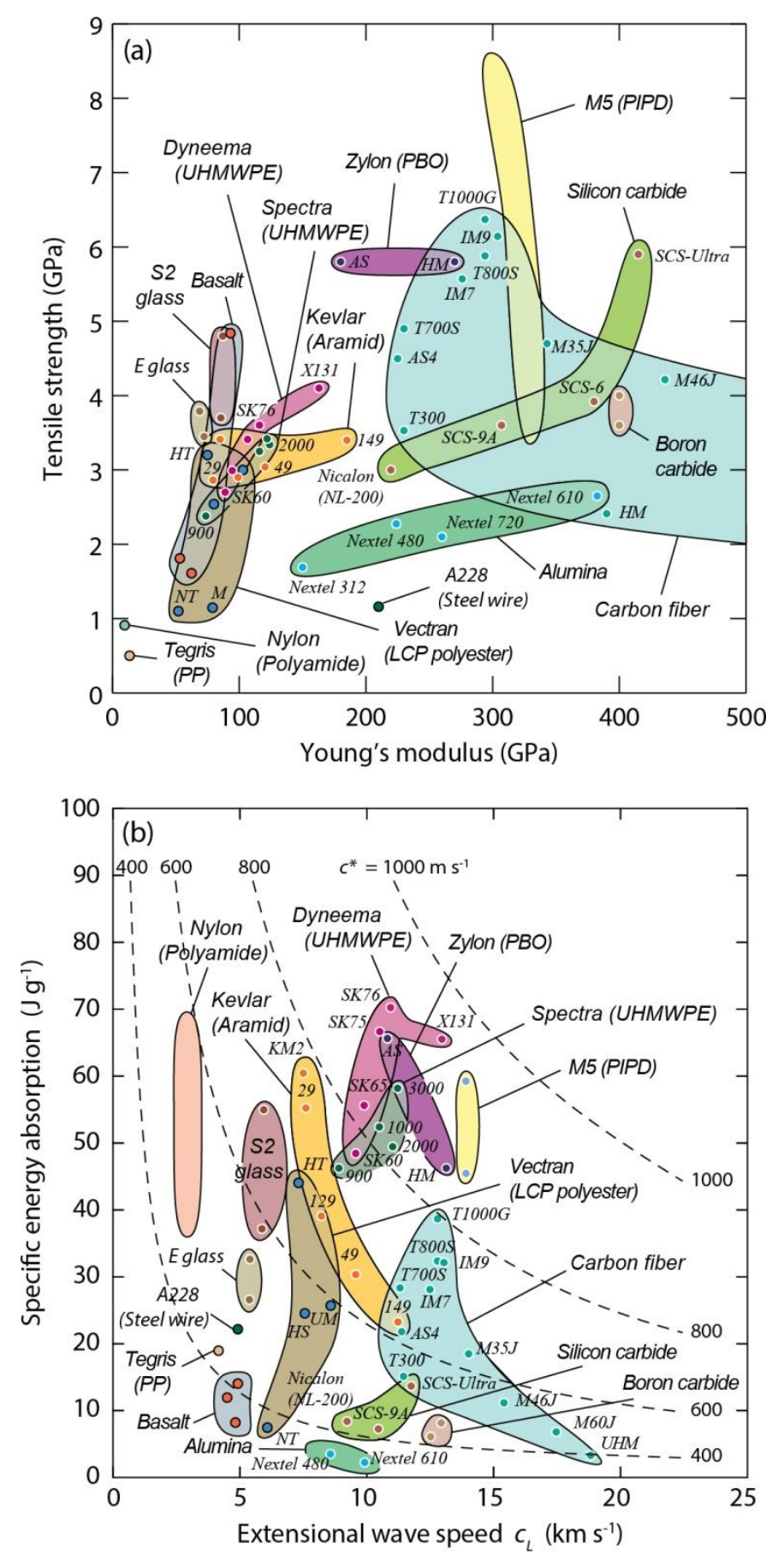

Figure 1.3. Material property charts comparing the (a) tensile strength and Young's modulus and (b) specific toughness and extensional wave speed, $c_{L}=(E / \rho)^{1 / 2}$, of high performance fibers. Contours of the Cunniff [24] velocity, $c^{*}$, are also plotted on (b).

Cunniff [24] has used scaling arguments to rationalize the selection of ballistic resistant fibers. Cunniff argued that the ballistic limit of a fiber, and their composites, 
scales linearly with a merit (material property) index called the Cunniff Index, $c^{*}$, which has dimensions of $[\mathrm{m}][\mathrm{s}]^{-1}$. The ballistic limit being defined as the minimum impact velocity to fully perforate a target. For linear elastic fibers, it can be expressed as:

$$
c^{*}=\left[\frac{\sigma_{f} \varepsilon_{f}}{2 \rho}\left(\frac{E}{\rho}\right)^{1 / 2}\right]^{1 / 3}
$$

Where $\sigma_{f}$ is the failure strength, $\varepsilon_{f}$ is the strain-to-failure, E is the tensile Young's modulus and $\rho$ is the density of the fiber. The two material properties that make up the definition of $c^{*}$ are the fiber specific toughness and extensional wave speed, $c_{L}$. A material property map with axes of these two properties for various high-performance fibers is given in Figure 1.3(b), along with overlaid contours of constant $c^{*}$ values. Based on the Cunniff Index, the three highest ballistic limit materials are all polymers: SK76 Dyneema ${ }^{\circledR}$ (an UHMWPE material), Zylon and the M5 fiber. The M5 fiber remains under commercial development, and Zylon is susceptible to environmental degradation [25]. This dissertation investigates high Cunniff Index UHMWPE fibers that are combined with compliant matrices to form cross-ply laminated composites.

\subsection{Impact response of polymer matrix composites}

UHMWPE fiber reinforced polymer matrix composites (PMC) have been shown to exhibit remarkable resistance to penetration during localized impact loading by blunt projectiles $[24,26-30]$. The performance is dependent upon the composite architecture as well as fiber and (to lesser extent) matrix properties [26,30-33]. The focus of much research therefore has been in exploring the mechanisms responsible for projectile penetration in the velocity regime below where hydrodynamic effects dominate 
[22,30,34-39]. Such measurements have been used to develop continuum models (e.g. $[35,40,41])$ to enable the modelling of the penetration resistance, but these typically have a narrow range of validity in terms of projectile type, armor geometry, etc. and more importantly give little insight into the physical basis of the scaling relation proposed by Cunniff [24].

In an elegant analytical study, Phoenix and Porwal [42] showed that the ballistic limit of composite plates can scale with $c^{*}$ by assuming a membrane stretching mode of deformation is activated by projectile impact with a $\left[0^{\circ} / 90^{\circ}\right]$ composite plate as illustrated in Figure 1.4. When a cross-ply laminate is centrally impacted (at zero obliquity) by a projectile, it exerts a pressure (which scales with the product of the laminate density with the square of the impact velocity, $V_{i}$ ) that induces deflection, with concomitant stretching once the out of plane deflection exceeds the laminate thickness; a phenomenon that Cunniff [43] related to single fiber impact studies by Smith and collaborators [18]. The impact excites longitudinal (extensional) wavelets in the fibers directly beneath the projectile (termed 'primary fibers') that propagate away from the projectile impact site at the longitudinal wave speed, $c_{L}$. These elastically extend the fiber, and enable material motion towards the point of impact, Figure 1.4(b). A slower shear wave with a displacement vertical to the original laminate surface, travels more slowly behind the extensional wave, and facilitates out-of-plane motion of this strained material. The deflection of the primary fibers also induces transverse motion (and straining) of 'secondary' fibers (those not directly beneath the projectile). Due to the anisotropy of the $\left[0^{\circ} / 90^{\circ}\right]$ laminate, a pyramidal shaped transverse deflection develops, whose base is bounded by the orientation dependent transverse hinge speed, $c_{H}$. By 
analyzing this deflection in the thin membrane limit, Phoenix and Porwall [42] were able to predict the tensile stress in the fibers. The model then assumed laminate failure to be a binary event that was activated when the tensile stress in the stretched laminate reached the tensile strength of the fibers.

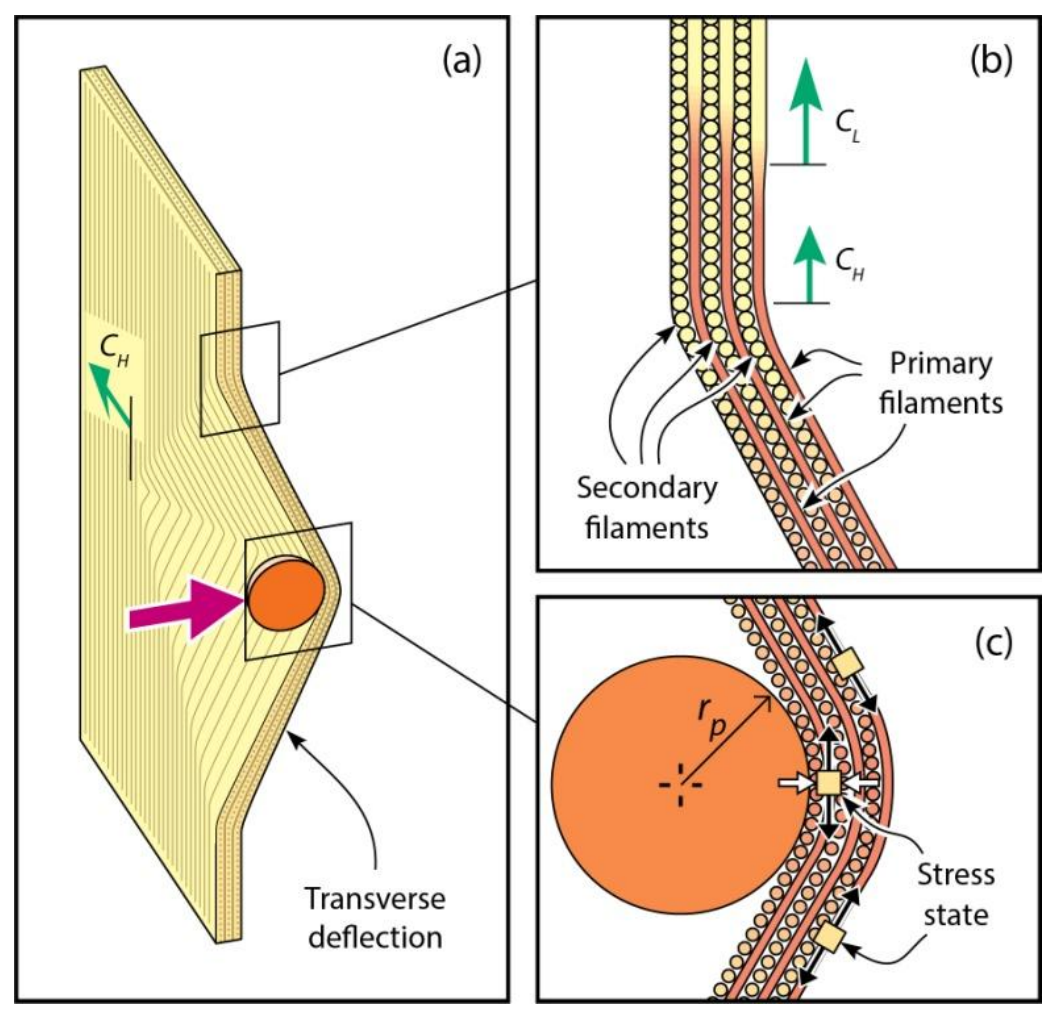

Figure 1.4. (a) Schematic illustration of the deformation mechanisms occurring during the impact of a $\left[0^{\circ} / 90^{\circ}\right]_{3}$ fiber reinforced composite laminate. Magnified views that show $(b)$ the wave propagation along the laminate and $(c)$ the stress state under the projectile.

A recent experimental investigation by Karthikeyan et al. [30] investigated the transverse deflection dynamics and impact site damage mechanisms of a $6 \mathrm{~mm}$ thick, $\left[0^{\circ} / 90^{\circ}\right]_{48}$, Dyneema ${ }^{\circledR} \mathrm{HB} 26$ (SK76 fiber reinforced) plate impacted by a $12.7 \mathrm{~mm}$ diameter hardened steel spherical projectile. The plates had an areal density of $5.89 \mathrm{~kg} \mathrm{~m}^{-}$ ${ }^{2}$, and were bolted between annular steel plates with an inner radius of $100 \mathrm{~mm}$. The ballistic limit (impact velocity needed to penetrate the full thickness of a laminate) was 
measured and found to be about $445 \mathrm{~m} \mathrm{~s}^{-1}$. They confirmed the formation of a pyramidal deflection structure following impact Figure 1.2(a), and measured the transverse hinge velocity in the fiber directions, $c_{H} \approx 316 \mathrm{~m} \mathrm{~s}^{-1}$. However, examination of the laminate after impact revealed that penetration of the laminate was progressive, with the number of failed plies increasing with impact velocity. No plug formation was observed, and the observation of progressive failure appeared to be inconsistent with the Phoenix and Porwall [42] model of binary failure in a membrane-stretching mode (a non-progressive mode of failure).

This progressive mechanism of penetration is often observed during high velocity impact of edge restrained panels $[27,30,38,44-46]$, and it is especially evident for very thick panels impacted at zero obliquity [47]. Woodward et al. [48] have suggested an alternate failure mechanism for fiber composites by investigating their response under indentation type loading. They argue that the compressed laminate under the indenter is stretched but unable to flow laterally (confined) due to the surrounding laminate material. The stress field underneath the punch intensifies with indentation depth until the group of plies closest to the punch, which are stretched the greatest, fail in tension (Figure 1.2(c)). While the mechanics underlying the hypothesis of Woodward et al. [48] are not clear, the sequence of events leading to failure suggested by Woodward et al. [48] more closely resembles the observations of Karthikeyan et al. [30].

In a recent submitted paper with Attwood et al. [49], we conducted a series of quasi-static compression tests on polymer fiber reinforced composite laminates to investigate the compressive response of these polymer composites. We discovered the existence of an indirect tension mode of ply failure when cross-ply laminates were 
compressed (the mechanism was absent in unidirectional composites as shown in Section 3.5). Ply tension in the fiber direction of each ply was shown to be activated by lateral expansion of the orthogonal plies above and below them; a consequence of the very large anisotropy of the Poisson expansion parallel and transverse to the ply fiber direction, Figure 1.5. As a result, during out of plane compression of cross-ply laminates, the fibers become loaded in tension by a shear lag mechanism, Figure 1.5(b). It will be shown later that when the tensile stress in a ply reaches the ply tensile strength, unstable ply fracture occurs. The critical pressure for ply failure was shown to be sensitive to the ply thickness, the tensile strength of the plies, the interlaminar shear strength of the laminate and its dependence on compressive loading. 
(a)

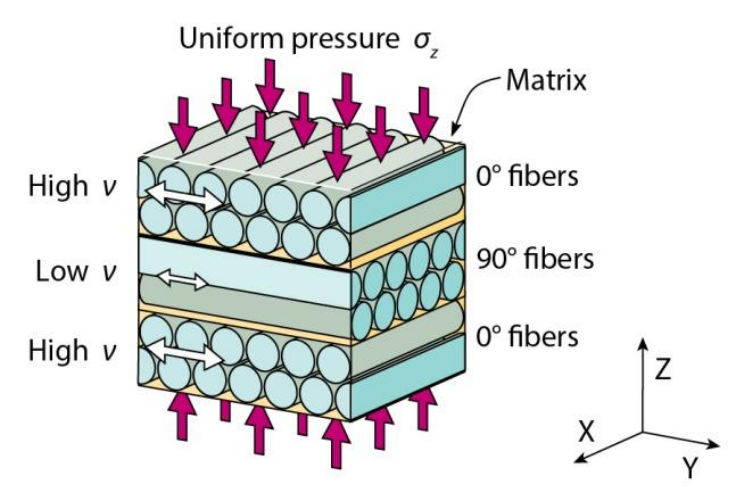

(b)
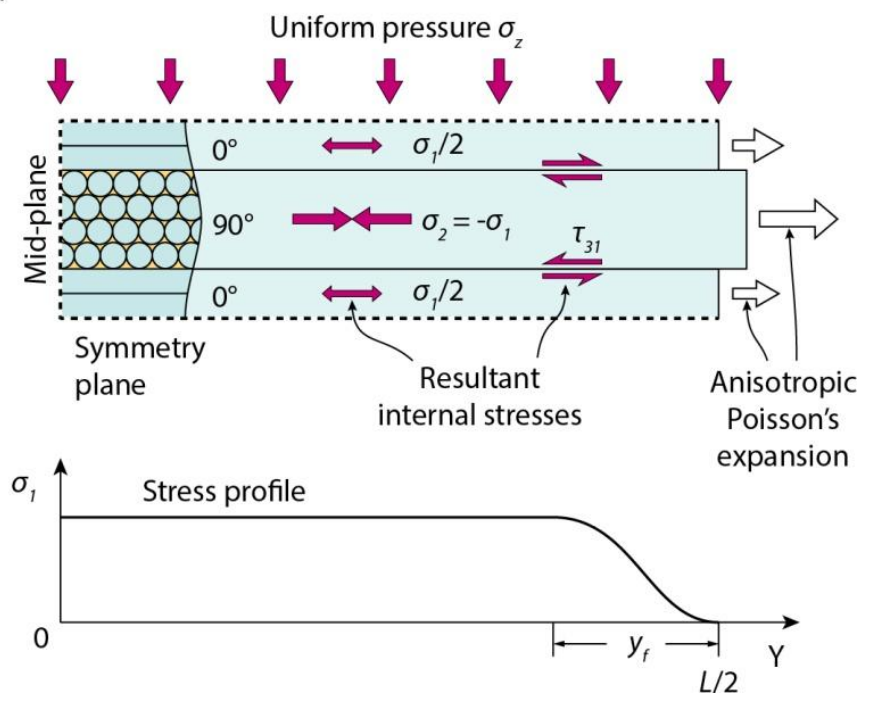

Figure 1.5. (a) Schematic illustration of plies within a cross-ply laminate under a uniform compressive stress, $\sigma_{z}$. Poisson lateral expansion in the fiber direction is much less than that transverse to the fibers. (b) Schematic illustrations of the stress within the composite predicted by the shear lag model.

Another mechanism that has been proposed as being active during penetration of a laminate is the shearing of fibers at the edge of, or underneath, the projectile $[26,50-$ 52]. While this mechanism may be of contribution with sharp nosed projectiles, this claim appears to be a generalization from observations of impacts of rigid fibers (e.g. glass and carbon) embedded in strong, brittle matrices (e.g. epoxy). In these brittle composite systems, cone cracks develop below the projectile by a shear mechanism $[30,48,53]$. Indentation and ballistic impact experiments $[30,48,54]$ with flat nose and spherical nosed loading surfaces expose a clear difference in penetration induced failure 
between the two systems, where no shear plug is created when a flexible polymer fiber reinforced composite (like those reinforced with UHMWPE fibers) fails under a ballistic impact loading.

The focus of this dissertation is to elucidate the different mechanisms of perforation and failure of UHMWPE fiber composite cross-ply laminates by impact scenarios and laboratory tests designed to reveal the fundamental micromechanisms.

\subsection{Dissertation goals}

This dissertation was written with the aim to better understand UHMWPE fiber reinforced composite failure behavior due to a dynamic impact. The first goal of the dissertation is to address the impact performance of the laminate in relation to the impulsive loading conditions with the ultimate goal of identifying the mechanisms controlling penetration. A second goal is to improve understanding of the compression induced indirect tension failure mechanism including the effect of defects up on its activation pressure. The existence of this mechanism will be addressed in the context of impact loading situations. The third goal is to see if the fundamental understanding that emerges from the first two goals can be used to design a model impact target that improves the impact performance a laminate.

\subsection{Dissertation outline}

Chapter 2 introduces the UHMWPE materials that are investigated in the dissertation. Chapter 3 reviews the measurement of their mechanical properties. Chapter 
4 presents an impact experiment using a model target designed to investigate the penetration behavior of an UHMWPE fiber polymer composite under various impact scenarios, where a spherical projectile impacts a laminate that is either back supported or edge gripped. In the latter case, the effects of pre-accelerating the laminate and fragmenting the projectile prior to impact are revealed. Chapter 5 presents an investigation and extension of the indirect tension model proposed in the Atwood et al. study [49]. Pervasive laminate defects are shown to be the cause of significant reductions in compressive strength, with implications for ballistic performance. In Chapter 6, a second model target is designed, using the fundamental insights gained in earlier parts of the dissertation, to delay activation of the indirect tension mechanism. It will be shown that this substantially increases its impact performance. A comprehensive discussion of the results and their implications is given in Chapter 7. Concluding remarks (Chapter 8) address the penetration of polymer matrix composites. Appendices A-C provide supplemental material in support of the dissertation.

\subsection{References}

[1] Rodriguez F, Cohen C, Ober CK, Archer LA. Principles of Polymer Systems. Fifth. New York: Taylor \& Francis; 2003.

[2] Afshari M, Sikkema DJ, Lee K, Bogle M. High Performance Fibers Based on Rigid and Flexible Polymers. Polym Rev 2008;48:230-74.

[3] Wunderlich B, Czornyj G. A Study of Equilibrium Melting of Polyethylene. Macromolecules 1977;10:906-13.

[4] Smith P, Lemstra PJ, Kalb B, Pennings AJ. Ultrahigh-strength polyethylene filaments by solution spinning and hot drawing. Polym Bull 1979;1:733-6.

[5] Smith P, Lemstra PJ. Ultra-high-strength polyethylene filaments by solution spinning/drawing. J Mater Sci 1980;15:505-14.

[6] Smith P, Lemstra PJ. Process for making polymer filaments which have a high tensile strength and a high modulus. US4344908A, 1982. 
[7] Anandakumaran K, Roy SK, Manley RSJ. Drawing-induced changes in the properties of polyethylene fibers prepared by gelation/crystallization. Macromolecules 1988;21:1746-51.

[8] Wang J, Smith Jr. KJ. The breaking strength of ultra-high molecular weight polyethylene fibers. Polymer 1999;40:7261-74.

[9] Fu Y, Chen W, Pyda M, Londono D, Annis B, Boller A, et al. Structure-property analysis for gel-spun, ultrahigh molecular mass polyethylene fibers. J Macromol Sci Part B 1996;35:37-87.

[10] Yeh J-T, Lin S-C, Tu C-W, Hsie K-H, Chang F-C. Investigation of the drawing mechanism of UHMWPE fibers. J Mater Sci 2008;43:4892-900.

[11] Kwon YK, Boller A, Pyda M, Wunderlich B. Melting and heat capacity of gelspun, ultra-high molar mass polyethylene fibers. Polymer 2000;41:6237-49.

[12] Litvinov VM, Xu J, Melian C, Demco DE, Möller M, Simmelink J. Morphology, Chain Dynamics, and Domain Sizes in Highly Drawn Gel-Spun Ultrahigh Molecular Weight Polyethylene Fibers at the Final Stages of Drawing by SAXS, WAXS, and 1H Solid-State NMR. Macromolecules 2011;44:9254-66.

[13] Sikkema DJ, Northolt MG, Pourdeyhimi B. Assessment of New High-Performance Fibers for Advanced Applications. MRS Bull 2003;28:579-84.

[14] Govaert LE, Peijs T. Tensile strength and work of fracture of oriented polyethylene fibre. Polymer 1995;36:4425-31.

[15] Vargas-Gonzalez LR, Walsh SM, Scott BR. Balancing ballistic and back-face deformation in helmets: the role of alternative resisns, fibers, and fiber architecture in mass-efficient head protection. In: Baker E, Templeton D, editors. Proc. 26th Int. Symp. Ballist., vol. 2, Miami, FL: DEStech Publications, Inc.; 2011, p. 1872-82.

[16] Hogg PJ. Composites in Armor. Science 2006;314:1100-1.

[17] Fink BK. Performance Metrics for Composite Integral Armor. J Thermoplast Compos Mater 2000;13:417-31.

[18] Smith JC, Fenstermaker CA, Shouse PJ. Behavior of Filamentous Materials Subjected to High-Speed Tensile Impact. Symp. Dyn. Behav. Mater., West Conshohocken, PA: ASTM International; 1963, p. 47-69.

[19] Field JE, Sun Q. High-speed photographic study of impact on fibers and woven fabrics. Proc SPIE 1991;1358:703-12.

[20] Carr DJ. Failure Mechanisms of Yarns Subjected to Ballistic Impact. J Mater Sci Lett 1999;18:585-8.

[21] Song B, Park H, Lu W-Y, Chen W. Transverse Impact Response of a Linear Elastic Ballistic Fiber Yarn. J Appl Mech 2011;78:051023-1-9.

[22] Chocron S, Kirchdoerfer T, King N, Freitas CJ. Modeling of Fabric Impact With High Speed Imaging and Nickel-Chromium Wires Validation. J Appl Mech 2011;78:051007-1-13. 
[23] Walker JD, Chocron S. Why Impacted Yarns Break at Lower Speed Than Classical Theory Predicts. J Appl Mech 2011;78:051021-1-7.

[24] Cunniff PM. Dimensionless parameters for optimization of textile-based body armor systems. In: Reinecke WG, editor. Proc. 18th Int. Symp. Ballist., San Antonio, TX: Technomic Publishing Company, Inc; 1999, p. 1303-10.

[25] National Institute of Justice. Third Status Report to the Attorney General on Body Armor Safety Initiative Testing and Activities. NIJ; 2005.

[26] Lee BL, Song JW, Ward JE. Failure of Spectra ${ }^{\circledR}$ Polyethylene Fiber-Reinforced Composites under Ballistic Impact Loading. J Compos Mater 1994;28:1202-26.

[27] Iremonger MJ. Polyethylene composites for protection against high velocity small arms bullets. In: Reinecke WG, editor. Proc. 18th Int. Symp. Ballist., San Antonio, TX: 1999, p. 946-54.

[28] Morye SS, Hine PJ, Duckett RA, Bacon DJ, Ward IM. Modelling of the energy absorption by polymer composites upon ballistic impact. Compos Sci Technol 2000;60:2631-42.

[29] Jacobs MJN, Dingenen JLJV. Ballistic protection mechanisms in personal armour. J Mater Sci 2001;36:3137-42.

[30] Karthikeyan K, Russell BP, Fleck NA, Wadley HNG, Deshpande VS. The effect of shear strength on the ballistic response of laminated composite plates. Eur J Mech ASolids 2013;42:35-53.

[31] Vargas-Gonzalez L, Walsh SM, Wolbert J. Impact and Ballistic Response of Hybridized Thermoplastic Laminates. Aberdeen Proving Ground, MD: Army Research Laboratory; 2011.

[32] Vargas-Gonzalez LR, Walsh SM, Gurganus JC. Examining the Relationship Between Ballistic and Structural Properties of Lightweight Thermoplastic Unidirectional Composite Laminates. Aberdeen Proving Ground, MD: Army Research Laboratory; 2011.

[33] Lee BL, Walsh TF, Won ST, Patts HM, Song JW, Mayer AH. Penetration Failure Mechanisms of Armor-Grade Fiber Composites under Impact. J Compos Mater 2001;35:1605-33.

[34] Cheeseman BA, Bogetti TA. Ballistic impact into fabric and compliant composite laminates. Compos Struct 2003;61:161-73.

[35] Grujicic M, Arakere G, He T, Bell WC, Glomski PS, Cheeseman BA. Multi-scale ballistic material modeling of cross-plied compliant composites. Compos Part B Eng 2009;40:468-82.

[36] Parsons EM, Weerasooriya T, Sarva S, Socrate S. Impact of woven fabric: Experiments and mesostructure-based continuum-level simulations. J Mech Phys Solids 2010;58:1995-2021. 
[37] Chocron S, King N, Bigger R, Walker JD, Heisserer U, van der Werff H. Impacts and Waves in Dyneema ${ }^{\circledR}$ HB80 Strips and Laminates. J Appl Mech 2013;80:031806-1-10.

[38] Greenhalgh ES, Bloodworth VM, Iannucci L, Pope D. Fractographic observations on Dyneema ${ }^{\circledR}$ composites under ballistic impact. Compos Part Appl Sci Manuf 2013;44:51-62.

[39] Karthikeyan K, Russell BP, Fleck NA, O’Masta MR, Wadley HNG, Deshpande VS. The soft impact response of composite laminate beams. Int J Impact Eng 2013;60:24-36.

[40] Grujicic M, Glomski PS, He T, Arakere G, Bell WC, Cheeseman BA. Material Modeling and Ballistic-Resistance Analysis of Armor-Grade Composites Reinforced with High-Performance Fibers. J Mater Eng Perform 2009;18:1169-82.

[41] Iannucci L, Pope D. High velocity impact and armour design. Express Polym Lett 2011;5:262-72.

[42] Phoenix SL, Porwal PK. A new membrane model for the ballistic impact response and V50 performance of multi-ply fibrous systems. Int J Solids Struct 2003;40:6723-65.

[43] Cunniff PM. An analysis of the system effects in woven fabrics under ballistic impact. Text Res J 1992;62:495-509.

[44] Scott BR. The penetration of compliant laminates by compact projectiles. Proc. 18th Int. Symp. Ballist., San Antonio, TX: 1999, p. 1184-91.

[45] Zhang Z, Shen S, Huancheng S, Zhang D. Ballistic Penetration of Dyneema Fiber Laminate. J Mater Sci Technol 1998;14:265-8.

[46] Grujicic M, Arakere G, He T, Bell WC, Cheeseman BA, Yen C-F, et al. A ballistic material model for cross-plied unidirectional ultra-high molecular-weight polyethylene fiber-reinforced armor-grade composites. Mater Sci Eng A 2008;498:231-41.

[47] Heisserer U, van der Werff H, Hendrix J. Ballistic Depth of Penetration Studies in Dyneema ${ }^{\circledR}$ Composites. In: Wickert M, Salk M, editors. Proc. 27th Int. Symp. Ballist., vol. 2, FREIBURG, GERMANY: DEStech Publications, Inc.; 2013, p. 1936-43.

[48] Woodward RL, Egglestone GT, Baxter BJ, Challis K. Resistance to penetration and compression of fibre-reinforced composite materials. Compos Eng 1994;4:329-41.

[49] Attwood JP, Khaderi S, Karthikeyan K, Fleck NA, O'Masta MR, Wadley HNG, et al. The out-of-plane compressive response of Dyneema composites. J Mech Phys Solids 2014:[Submitted for publication].

[50] Prosser RA, Cohen SH, Segars RA. Heat as a Factor in the Penetration of Cloth Ballistic Panels by 0.22 Caliber Projectiles. Text Res J 2000;70:709-22.

[51] Lim C., Tan VB., Cheong C. Perforation of high-strength double-ply fabric system by varying shaped projectiles. Int J Impact Eng 2002;27:577-91. 
[52] Taylor SA, Carr DJ. Post failure analysis of $0^{\circ} / 90^{\circ}$ ultra high molecular weight polyethylene composite after ballistic testing. J Microsc 1999;196:249-56.

[53] Gama BA, Gillespie Jr. JW. Punch shear based penetration model of ballistic impact of thick-section composites. Compos Struct 2008;86:356-69.

[54] Culnane AH, Woodward RL, Egglestone GT. Failure examination of composite materials using standard metallographic techniques. J Mater Sci Lett 1991;10:3334. 


\section{Chapter 2. UHMWPE fibers and composites}

\subsection{Processing, structure, and properties of UHMPWE fibers}

Polyethylene is the simplest hydrocarbon polymer. The molecule is assembled from the linear repetition of the monomer $\left(-\mathrm{CH}_{2}-\right)$, where the bonds between the carboncarbon $(\mathrm{C}-\mathrm{C})$ and carbon-hydrogen $(\mathrm{C}-\mathrm{H})$ atoms are covalent and are based on $\mathrm{sp}^{3}$ hybridization of the carbon valence electrons [1]. This results in a $\mathrm{C}-\mathrm{C}$ bond length of $1.55 \AA$ and C-H length of $1.09 \AA$ A, a C-C-C bond angle of $112^{\circ}$ and an $\mathrm{H}-\mathrm{C}-\mathrm{H}$ angle of $108^{\circ}$ [2]. The typical 'zig-zag' trans-conformation of the molecule is shown in Figure 2.1(a). This is the most stable conformation, but the low steric hindrance (activation energy of $\sim 12 \mathrm{~kJ} \mathrm{~mol}^{-1}$ ) allows portions of the molecule to easily rotate about its $\mathrm{C}-\mathrm{C}$ axis providing the molecule with the ability to bend and flex [1]. Bonding between neighboring molecules is by much weaker van der Waals bonds $\left(\sim 1 / 30^{\text {th }}\right.$ of the covalent bond strength) resulting from induced dipole moments between neighboring atoms [1]. 
This very weak intermolecular bonding is responsible for polyethylene's flexibility and softness.

The first high-performance extended chain configuration polymer fiber was made by controlling the molecular assembly of UHMWPE [3]. The absence of side chains and small cross-sectional area of the molecular chain, enable UHMWPE to be packed into an orthorhombic crystal lattice structure, Figure 2.1(a) [4,5]. This phase can be assembled from two macro-conformations: i) a folded-chain lamellar structure and ii) an extendedchain structure, Figure 2.1(c) and (d) [6-9]. In the extended-chain macro-conformation, the full length of an individual molecule can be aligned in the fiber direction with weak van der Waals forces binding hydrogen atoms between neighboring extended carbon chains. The internal cohesive energy between the carbon atoms in these long chain molecules $\left(\sim 0.1 \mu \mathrm{m}\right.$ in length per $10,000 \mathrm{~g} \mathrm{~mol}^{-1}$ in molecular weight $)$ is orders of magnitude greater than the $\mathrm{C}-\mathrm{C}$ bond energy $\left(330 \mathrm{~kJ} \mathrm{~mol}^{-1}\right)$ and immobilizes the molecules in the ordered crystal formation [10]. 
(a) Orthorhomic unit cell

(b) Fiber texturing of crystal structure

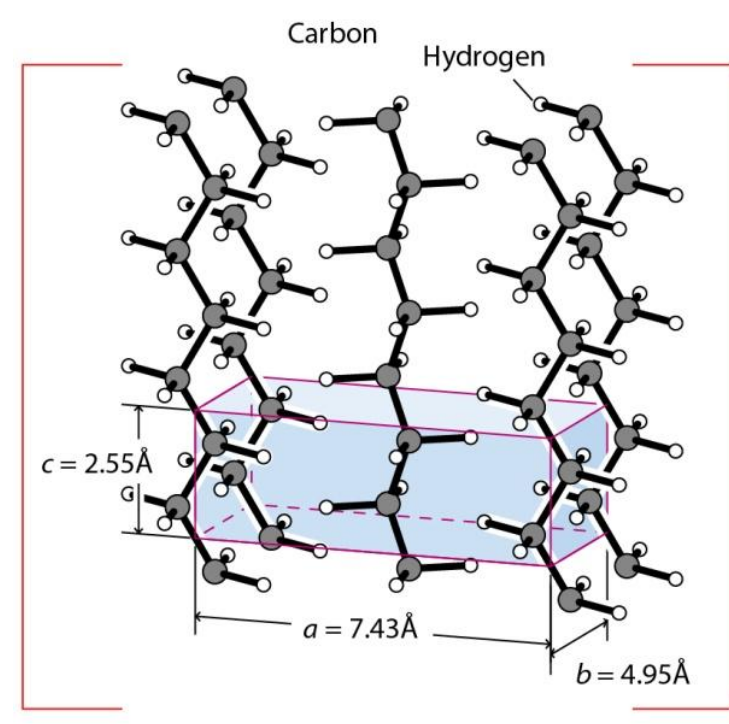

(c) Folded-chain macroconformation

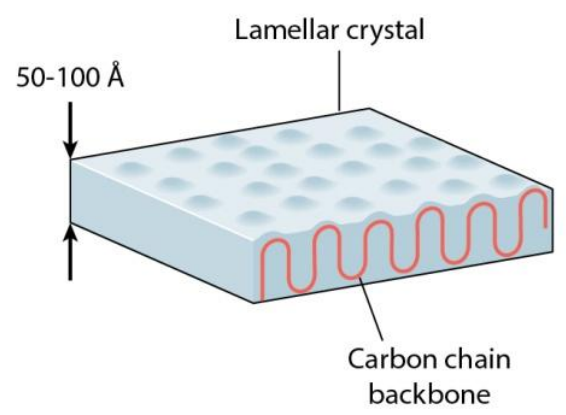

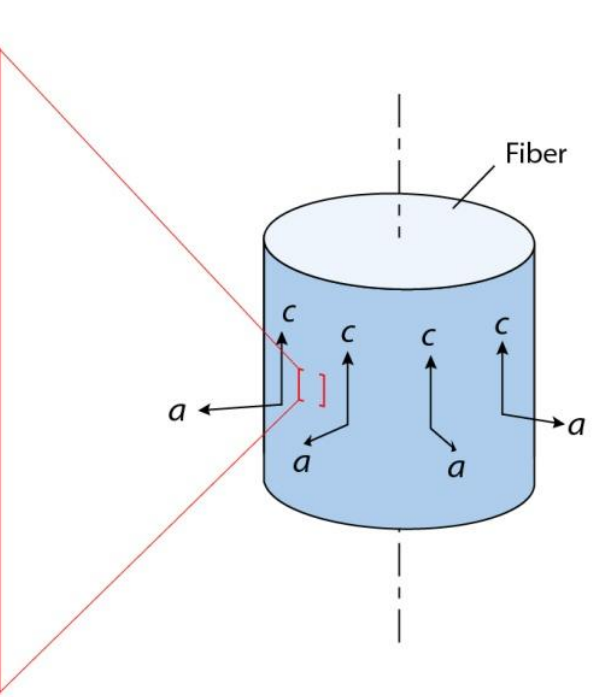

(d) Extended-chain macroconformation

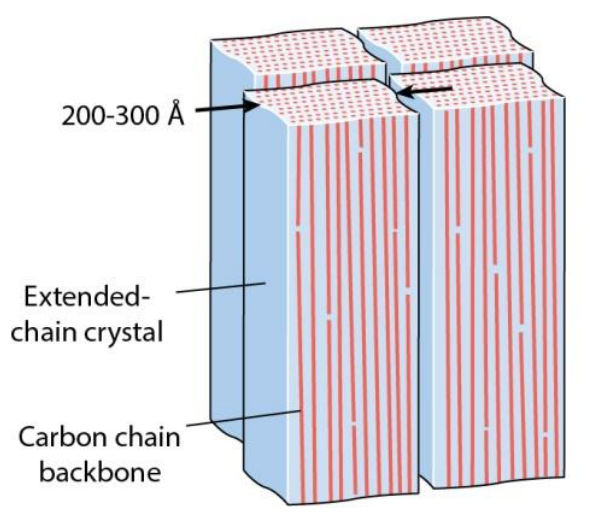

Figure 2.1. (a) Packing of polyethylene molecules in an orthorhombic crystal structure. (b) Crystal texturing in an UHMWPE drawn fiber, where the molecules are oriented in the fiber orientation. This crystal structure can assemble as either (c) a folded-chain lamellar structure or (d) an extended-chain structure.

The theoretical strength can be estimated by differentiating the Morse potential to obtain the force required to break the $\mathrm{C}-\mathrm{C}$ covalent carbon bond $[4,11]$. This gives an unrealistic value $\sim 30 \mathrm{GPa}$. A density functional calculation by Hagemon et al. [12] gives a chain strength estimate of $18 \mathrm{GPa}$, which is about twice that of measurements. An alternative model by proposed Smith et al. [13] used thermodynamic arguments to estimate the tensile strength of a perfect crystalline fiber, Figure 2.1(d). They argued that 
increasing the tensile stress raises the chemical potential of the crystalline phase, which shifts its melt temperature to lower values. Therefore the fiber fails when the load is high enough to depress the melting temperature to ambient. This analysis gives an estimated tensile strength of 7-8 GPa and a modulus of 235-325 GPa. These values match well with laboratory scale tests that have measured strengths of $\sim 7 \mathrm{GPa}$ and moduli of $\sim 250 \mathrm{GPa}$ $[14,15]$. If these parameters are combined, they give an upper bound Cunniff Index, $c^{*} \approx$ $1200 \mathrm{~m} \mathrm{~s}^{-1}$ that is well above that of fibers commercially available today, Figure 1.3(b).

The fibers are typically produced by a gel-spinning process (Figure 2.2) originally patented by Paul Smith and Pieter Lemstra [5,16-18]. The process consists of three steps: 1) converting UHMWPE polymer to a gel, 2) converting the gel to a xerogel and 3) hot drawing. A solution, of UHMWPE granules dissolved in a solvent, is used to create a gel, Figure 2.2(a). Structurally, the gel is composed of $5 \mathrm{~nm}$ thick lamellar microcrystals of folded-chain orthorhombic crystals, Figure 2.2(b). In the second fiber gel spinning step, clusters of lamellar crystallites form a coplanar arrangement (a sandwich type structure), Figure 2.2(c). A small amount of the solvent is removed in this step. The hot-drawing step removes the remaining solvent and extends the folded-chain lamellar crystallites, forming the extended-chain macro-conformation (and aligning the carbon backbone with the fiber axis), Figure 2.2 (d). This final step aligns the crystal orientation and decreases the fiber diameter, leading to increases in the strength and modulus of the fiber $[5,9,19-$ 21]. Crystal orientation in these fibers routinely exceed $95 \%$ with $>85 \%$ crystalline phase volume fraction $[20,15,7,19,22,9]$. 
(a) Gel-spinning of UHMWPE

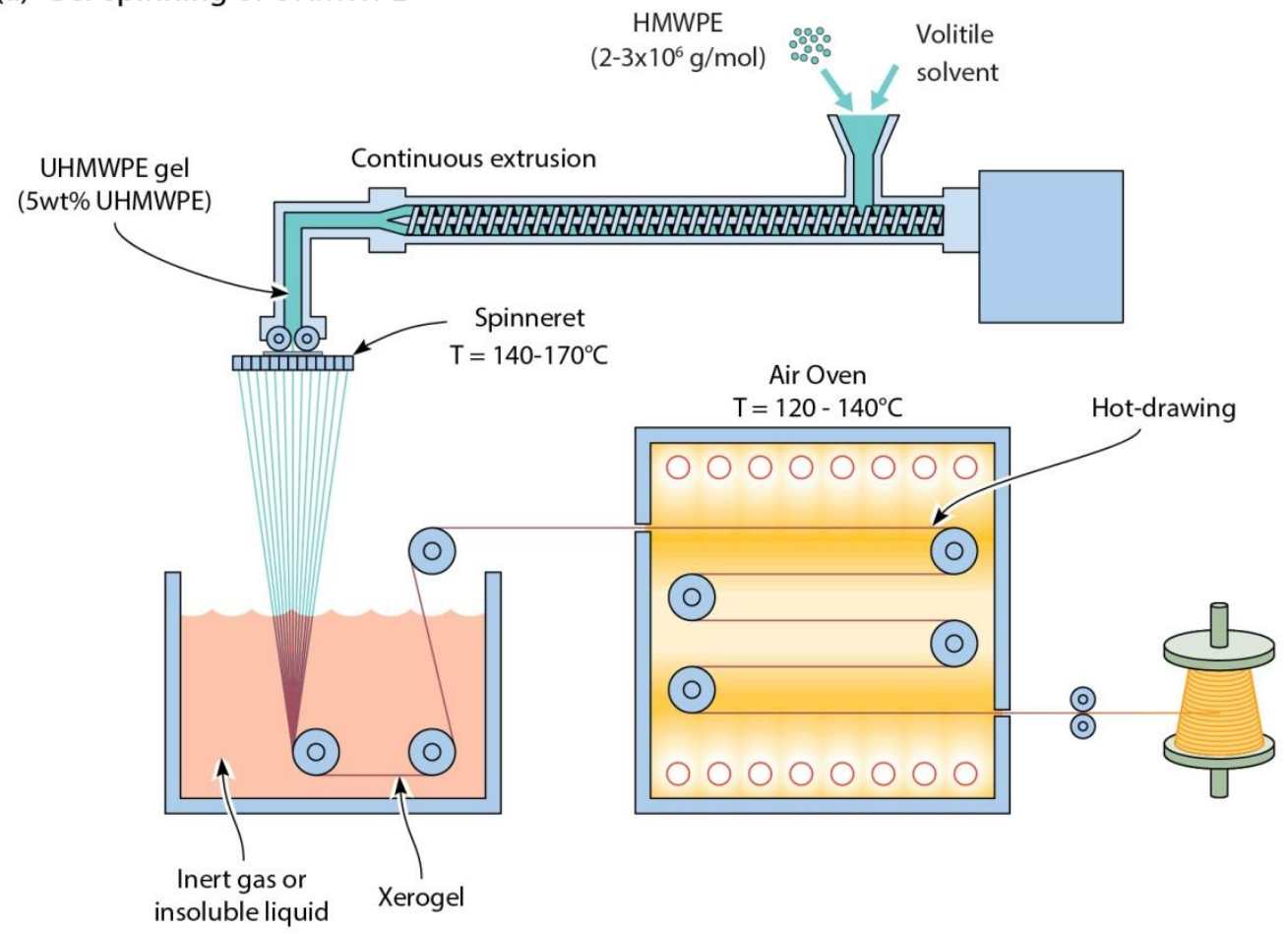

(b) UHMWPE gel

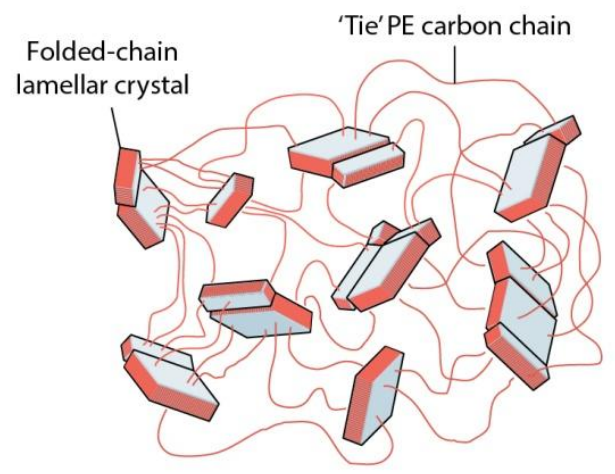

(c) Xerogel

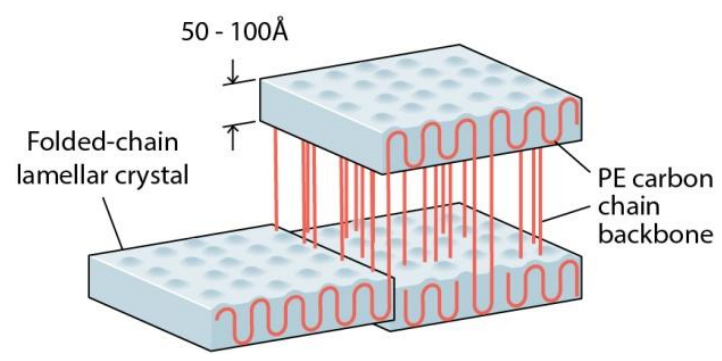

(d) Hot drawing

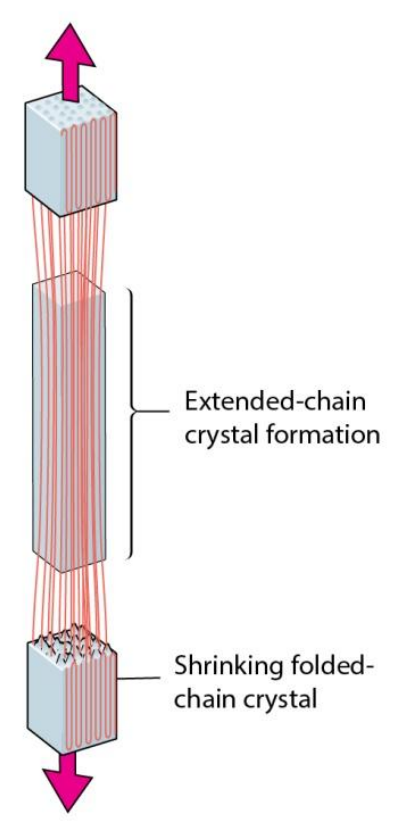

Figure 2.2. The (a) gel-spinning process used to make UHMWPE fibers. The (b) gel is composed of folded-chain lamellar crystals that are spun (with minimal removal of solvent) into (c) a Xerogel of coplanar lamellar crystals. Red line represents the carbon chain C-C backbone. (d) Hot drawing removes the remained of the solvent and extends the lamellar chains into a chainextended macro-conformation. 


\subsection{Investigated materials}

The commercially available UHMWPE fiber-reinforced laminates used in high intensity loading applications are Dyneema ${ }^{\circledR}$ (DSM; The Netherlands) and Spectra Shield $^{\circledR}$ (Honeywell; USA). Much of the dissertation will focus on Dyneema ${ }^{\circledR}$ and in particular upon the cross-ply laminate grade HB26, which is reinforced by a gel-spun fiber known as grade SK76. However, collaborators at DSM have provided other fibers for study including the SK90 and X131 grades. A previous investigative effort of the 17 $\mu \mathrm{m}$ SK76 fiber revealed it to be highly crystalline (80-90 vol.\%), with a highly textured orthorhombic crystal structure (a lattice parameter spacing of $\mathrm{a}=7.43 \AA$, $\mathrm{b}=4.95 \AA$ and $\mathrm{c}=2.53 \AA$ ) with the c-axis (molecular axis) aligned along the fiber direction, and predominantly in the extended chain macro-conformation [23]. Its as-manufactured tensile properties are shown in Table 2.1 together with those of the other two fiber types.

Table 2.1. DSM reported yarn tensile properties [From private communications].

\begin{tabular}{lccc}
\hline Yarn & $\begin{array}{c}\text { Strength } \\
(\mathrm{GPa})\end{array}$ & $\begin{array}{c}\text { Modulus } \\
(\mathrm{GPa})\end{array}$ & $\begin{array}{c}\text { Strain-to- } \\
\text { failure }(\%)\end{array}$ \\
\hline SK76 & 3.6 & 115 & 3.7 \\
SK90 & 3.8 & - & - \\
X131 & $4.1 \pm 0.2$ & $163 \pm 4$ & $3.1 \pm 0.1$ \\
\hline
\end{tabular}

To manufacture an HB26 laminate, a series of collinear yarns (bundles of 780 SK76 fibers) are used in a ply production process in which bundles of fibers are coated in a polyurethane resin solution to form a four fiber thick, unidirectional ply. Four of these plies are then assembled into a cross-ply $\left[0^{\circ} / 90^{\circ}\right]_{2}$ tape, the solvent is allowed to evaporate, and the tape is wound onto a $1.6 \mathrm{~m}$ wide roll. Strips of tape can then be cut from the roll, stacked and subjected to a hot-compaction procedure to form a laminate, 
Figure 1.1; as further discussed in Section 2.3. Micro X-ray computerized tomograms (micro-XCT) and scanning electron micrographs (SEM) of the cross section of a consolidated HB26 laminate are shown in Figure 2.3.
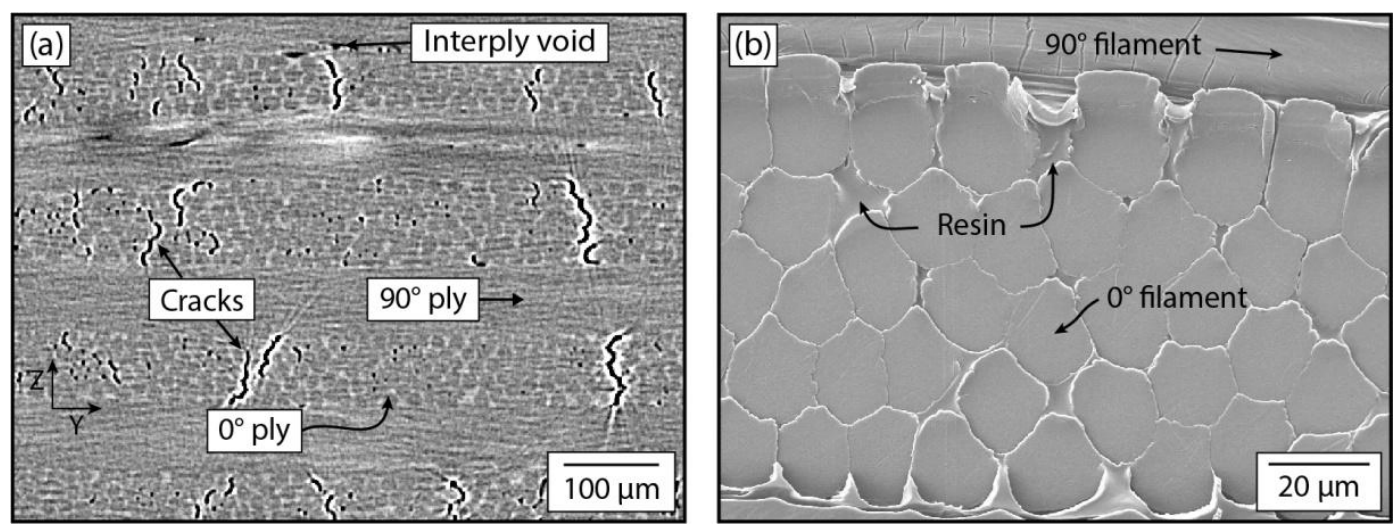

Figure 2.3. (a) XCT reconstruction of a consolidated HB26 laminate. (d) An SEM micrograph of a ply from a consolidated laminate cut normal to the filament direction. The $0^{\circ}$ filament ends were slightly smeared from the microtome blade.

To explore the generality of the phenomena discovered here, we also investigated five other UHMWPE fiber composite laminates. Each was composed of a compliant thermoplastic resin reinforced with a high volume fraction of UHMWPE fiber, and provided to us as in the form of $\left[0^{\circ} / 90^{\circ}\right]_{2}$ tape. Table 2.2 identifies the six sample types investigated in the study, and tabulates their constituent materials and architecture. The fiber diameter was measured using a scanning electron microscope, and the average ply thickness was calculated from the thickness of a 240 ply consolidated laminate measured with a digital caliper. Chapter 3 will discuss the remaining material properties shown in Table 2.2. 
Table 2.2. Constituent materials, architecture and mechanical properties of the various laminate grades under study

\begin{tabular}{|c|c|c|c|c|c|c|}
\hline & $\begin{array}{l}\text { Dyneema }^{\circledR} \\
\text { HB26 }\end{array}$ & $\begin{array}{l}\text { Dyneema }^{\circledR} \\
\text { HB50 }\end{array}$ & $\begin{array}{l}\text { Dyneema }^{\circledR} \\
\text { X106 }\end{array}$ & $\begin{array}{l}\text { Dyneema }^{\circledR} \\
\text { X131 }\end{array}$ & $\begin{array}{l}\text { Spectra } \\
\text { Shield }^{\circledR} \text { II } \\
\text { SR-3124 }\end{array}$ & $\begin{array}{l}\text { Spectra } \\
\text { Shield }^{\circledR} \text { II } \\
\text { SR-3136 }\end{array}$ \\
\hline Manufacturer & DSM & DSM & DSM & DSM & Honeywell & Honeywell \\
\hline Fiber Type & $\begin{array}{l}\text { Dyneema }^{\circledR-} \\
\text { SK76 }\end{array}$ & $\begin{array}{l}\text { Dyneema }^{\circledR-} \\
\text { SK76 }\end{array}$ & $\begin{array}{l}\text { Dyneema }^{\circledR-} \\
\text { SK90 }\end{array}$ & $\begin{array}{c}\text { Dyneema }^{\circledR} \\
\text { X131 }\end{array}$ & Spectra $^{\circledR}$ II & Spectra $^{\circledR}$ II \\
\hline Resin & PADP & SISTC & SISTC & SISTC & $\mathrm{PU}$ & SB \\
\hline Fiber diameter, $t(\mu \mathrm{m})$ & 17 & 17 & 10 & 13 & 26 & 29 \\
\hline Ply thickness, $h(\mu \mathrm{m})$ & 67 & 60 & 40 & 45 & 65 & 65 \\
\hline $\begin{array}{l}\text { Avg. number of stacked } \\
\text { fibers per ply thickness }\end{array}$ & 4.3 & 3.9 & 4.5 & 3.8 & 2.7 & 2.4 \\
\hline $\begin{array}{l}\text { Longitudinal ply modulus, } \\
E_{f}(\mathrm{GPa})\end{array}$ & 70 & 70 & 90 & 90 & 70 & 70 \\
\hline $\begin{array}{l}\text { Ply tensile strength, } \\
\sigma_{f}(\mathrm{GPa})\end{array}$ & 1.7 & 1.7 & 2.2 & 2.2 & 1.7 & 1.7 \\
\hline $\begin{array}{l}\text { Inter-laminar shear strength, } \\
\tau_{0}(\mathrm{MPa})\end{array}$ & 1.8 & 0.35 & 0.45 & 0.35 & 0.13 & 1.3 \\
\hline $\begin{array}{l}\text { Pressure sensitivity } \\
\text { coefficient, } \mu\end{array}$ & 0.065 & 0.065 & 0.050 & 0.055 & 0.065 & 0.065 \\
\hline
\end{tabular}

* Resin abbreviations: polyetherdiol-aliphatic diiocyanate polyurethane (PADP); styrene-isoprene-styrene triblock copolymer (SISTC); polyurethane based (PU); styrene based (SB) 
Dyneema ${ }^{\circledR}$ HB50 uses the same fiber as the HB26 grade, but a more compliant styrene-isoprene-styrene triblock copolymer (SISTC) resin for the matrix. The developmental, non-commercial, Dyneema ${ }^{\circledR}$ grades X106 and X131 used the same matrix as the HB50 grade, but were reinforced with higher strength (see Table 2.1), smaller diameter SK90 and X131 UHMWPE fibers, and thus had slightly thinner plies. Spectra Shield $^{\circledR}$ II grades SR3124 and SR3136 are similar to HB50 and HB26, respectively, but are manufactured by Honeywell using Spectra ${ }^{\circledR}$ II UHMWPE fibers. The grade SR3124 used a more compliant styrene based matrix, while SR3136 used a polyurethane based matrix.

\subsection{Laminate consolidation}

UHMWPE materials are difficult to attach to other materials. This arises from its surface chemical inertness (lack of polarity and functional groups), very low friction coefficient and low surface roughness [24-26]. The binding of a fiber to a matrix is a necessity for stress-transfer into the fibers through the fiber/matrix interface [27]. Various techniques can be used to improve adhesion which involve modifying the surface of the fibers, e.g. corona-discharge treatments and chemical oxidation [25,26,28]. However, they all reduce the fiber strength. For high-performance applications, binding of a fiber with a chemically similar resin is achieved by van der Waals bonding between hydrogen atoms on adjacent molecules at the fiber - matrix interface. For this to occur it is necessary to bring the two phases into close proximity.

Typically, sheets of the fiber/resin pre-preg are stacked and exposed to a low temperature, high pressure consolidation process. The temperature is raised above the 
flow temperature of the matrix to facilitate the distribution of the resin across the fibers and between plies, as well as reducing voids within the laminate (investigated in depth in Chapter 5). All laminates were consolidated by the following procedure unless otherwise stated: Sections of pre-preg tape were stacked to desired thickness and lay-up. The prepreg stack was then positioned between two platens that had been pre-heated to $100{ }^{\circ} \mathrm{C}$, and a pressure of $20.6 \mathrm{MPa}$ was applied. The temperature was increased to $127^{\circ} \mathrm{C}$ and maintained for 15 minutes. The die/sample assembly was then cooled to $90^{\circ} \mathrm{C}$, the pressure released and the sample allowed to cool to ambient. This method was chosen due to its use in the defense industry and recommendation by DSM.

\subsection{References}

[1] Maitland Jones. Organic Chemistry. 2nd ed. New York: W.W. Norton \& Compnay, Inc.; 2000.

[2] Förster G, Meister A, Blume A. Van der Waals energy contour map describing the orthorhombic hydrocarbon chain packing of symmetry Pbnm. Phys Chem Chem Phys 2000;2:4503-8.

[3] Committee on High Performance Synthetic Fibers for Composites, National Materials Advisory Board, Division on Engineering and Physical Sciences, National Research Council. High-Performance Structural Fibers for Advanced Polymer Matrix Composites. Washington, D.C.: The National Academies Press; 2005.

[4] Boudreaux DS. Calculations of the strength of the polyethylene molecule. J Polym Sci Polym Phys Ed 1973;11:1285-92.

[5] Afshari M, Sikkema DJ, Lee K, Bogle M. High Performance Fibers Based on Rigid and Flexible Polymers. Polym Rev 2008;48:230-74.

[6] Bassett DC, Turner B. On chain-extended and chainfolded crystallization of polyethylene. Philos Mag 1974;29:285-307.

[7] Fu Y, Chen W, Pyda M, Londono D, Annis B, Boller A, et al. Structure-property analysis for gel-spun, ultrahigh molecular mass polyethylene fibers. J Macromol Sci Part B 1996;35:37-87.

[8] Pak J, Wunderlich B. Reversible melting of gel-spun fibers of polyethylene. Thermochim Acta 2004;421:203-9. 
[9] Litvinov VM, Xu J, Melian C, Demco DE, Möller M, Simmelink J. Morphology, Chain Dynamics, and Domain Sizes in Highly Drawn Gel-Spun Ultrahigh Molecular Weight Polyethylene Fibers at the Final Stages of Drawing by SAXS, WAXS, and 1H Solid-State NMR. Macromolecules 2011;44:9254-66.

[10] Smith Jr. KJ, Wang J. The breaking strength of imperfect (real) polymer fibers. Polymer 1999;40:7251-60.

[11] Pennings AJ, Roukema M, Veen AV der. Further studies on the high-speed gelspinning of ultra-high molecular weight polyethylene. Polym Bull 1990;23:353-9.

[12] Hageman JCL, de Wijs GA, de Groot RA, Meier RJ. Bond Scission in a Perfect Polyethylene Chain and the Consequences for the Ultimate Strength. Macromolecules 2000;33:9098-108.

[13] Smith KJ. The breaking strength of perfect polymer fibers. Polym Eng Sci 1990;30:437-43.

[14] Werff $\mathrm{H}$ van der, Pennings AJ. Tensile deformation of high strength and high modulus polyethylene fibers. Colloid Polym Sci 1991;269:747-63.

[15] Wang J, Smith Jr. KJ. The breaking strength of ultra-high molecular weight polyethylene fibers. Polymer 1999;40:7261-74.

[16] Smith P, Lemstra PJ, Kalb B, Pennings AJ. Ultrahigh-strength polyethylene filaments by solution spinning and hot drawing. Polym Bull 1979;1:733-6.

[17] Smith P, Lemstra PJ. Ultra-high-strength polyethylene filaments by solution spinning/drawing. J Mater Sci 1980;15:505-14.

[18] Smith P, Lemstra PJ. Process for making polymer filaments which have a high tensile strength and a high modulus. US4344908A, 1982.

[19] Yeh J-T, Lin S-C, Tu C-W, Hsie K-H, Chang F-C. Investigation of the drawing mechanism of UHMWPE fibers. J Mater Sci 2008;43:4892-900.

[20] Anandakumaran K, Roy SK, Manley RSJ. Drawing-induced changes in the properties of polyethylene fibers prepared by gelation/crystallization. Macromolecules 1988;21:1746-51.

[21] Pakhomov PM, Khizhnyak SD, Golikova AY, Galitsyn VP. Structural transformations in the course of gel spinning of high-strength polymer fibers. Phys Solid State 2005;47:1028-33.

[22] Kwon YK, Boller A, Pyda M, Wunderlich B. Melting and heat capacity of gelspun, ultra-high molar mass polyethylene fibers. Polymer 2000;41:6237-49.

[23] O'Masta MR. Ultra-high molecular-weight polyethylene composites: structure, properties and ballistic response. Master's Thesis. University of Virginia, 2010.

[24] Cohen Y, Rein DM, Vaykhansky L. A novel composite based on ultra-highmolecular-weight polyethylene. Compos Sci Technol 1997;57:1149-54.

[25] Devaux E, Cazé C. Composites of UHMW polyethylene fibres in a LD polyethylene matrix. I. Processing conditions. Compos Sci Technol 1999;59:459_ 66. 
[26] Chiu H-T, Wang J-H. The relationship between zeta-potential and pull-out shear strength on modified UHMWPE fiber reinforced epoxy composites. Polym Compos 1998;19:347-51.

[27] Gibson RF. Principles of composite material mechanics. 2nd ed. Boca Raton: CRC Press; 2007.

[28] Ogawa T, Mukai H, Osawa S. Improvement of the mechanical properties of an ultrahigh molecular weight polyethylene fiber/epoxy composite by corona-discharge treatment. J Appl Polym Sci 2001;79:1162-8. 


\section{Chapter 3. Fiber and composite mechanical response}

This chapter presents the measured mechanical properties of the UHMWPE materials. Only those properties that will be referenced in the subsequent chapters are presented and discussed. UHMWPE fibers and the reinforced composite laminates made from them are highly anisotropic, with tensile properties in the fiber direction orders of magnitude greater than in the transverse direction (or in flexure and shear) [1-5]. Russell et al. [1] have shown the tensile response of a $\left[0^{\circ} / 90^{\circ}\right]$ laminate is controlled by that of the fiber and was $~ 800 \mathrm{MPa}$ for the HB26 system. Karthikeyan et al. [2] measured laminated composites with fibers at $\pm 45^{\circ}$ to the tension axis to determine the in-plane shear response of the matrix. The matrix shear failure strength was $\sim 15 \mathrm{MPa}$ for the HB26 system, but only 1.5 MPa for HB50 which used a different (softer) matrix.

Here, the tensile response of Dyneema ${ }^{\circledR}$ yarns (bundles of fibers) is measured first and compared to the DSM reported fiber data. The tensile and inter-laminar shear strengths of the Dyneema ${ }^{\circledR}$ and Spectra Shield ${ }^{\circledR}$ II laminates investigated later are then 
reported. An initial exploratory study of the dependence of inter-laminar shear strength on through-thickness compression stress is also presented for the Dyneema ${ }^{\circledR} \mathrm{HB} 26$ composite. This has been investigated because a strong dependence of shear strength upon transverse compression stress has been reported for bulk polymers [6] and is utilized in the indirect tension model of Attwood et al. [5]. The indirect tension model also assumes that the Poisson ratio of a laminate is highly anisotropic, and so the Poisson ratio of a unidirectional, $\left[0^{\circ}\right]_{45}$, HB26 laminate has been measured both parallel and transverse to the fiber direction during out of plane compression. All the measurements have been made under quasi-static loading. Russell et al. [1] have shown that the fiber strength is independent of displacement rate, and in this dissertation the assumption is implicitly carried forward to laminate properties.

\subsection{Fiber tensile response}

\subsubsection{Methodology}

The very low shear strength and friction coefficient of UHMWPE fibers makes it difficult to grip samples during mechanical testing. Russell et al. [1] developed a means for doing this with single fibers, and showed that the ambient temperature tensile stress strain response of the SK76 fiber was strain rate insensitive over the strain rate range $10^{-2}$ $\mathrm{s}^{-1}$ to $10^{3} \mathrm{~s}^{-1}$. Therefore the tensile responses of yarns of Dyneema ${ }^{\circledR}$ SK76 and X131 measured at low rates and ambient temperature are representative of their properties when impacted by a projectile. The fiber strength tests performed here were conducted by sandwiching the ends of a yarn between two pieces of highly adherent tape, and clamping the taped yarn ends in wedge-action grips equipped with serrated jaw faces. An Instron 
(Norwood, MA) model 4208 screw-driven mechanical testing machine equipped with a $2 \mathrm{kN}$ load cell was then used to extend $100 \mathrm{~mm}$ long yarns at a nominal strain rate of $10^{-2} \mathrm{~s}^{-1}$

\subsubsection{Results}

A typical measured tensile stress versus applied nominal strain response for the SK76 yarn is plotted in Figure 3.1. The response is consistent with that of a large body of previous studies (see for example [1,7-9]). The tensile response is elastic-brittle with a tensile elastic (Young's) modulus of approximately $110 \mathrm{GPa}$ and a tensile strength of approximately 3.1 GPa. A similar response was seen for the X131 yarn. The average strengths of 20 repeated tests were $3.2 \pm 0.2 \mathrm{GPa}$ for the SK76 and $3.9 \pm 0.2 \mathrm{GPa}$ for the X131 yarn. This indicates that SK76 reinforced laminates such as HB26 and HB50, will have a lower tensile strength than an X131 laminate if laminate strength is fiber dominated. We also note that the strength values for a yarn are lower than those reported for a fiber (Table 2.1). Fiber misalignment with the loading axis and fiber waviness within the yarn leads to uneven loading of individual fibers in a yarn. Some fibers will then carry a disproportionate share of the load, and prematurely fail. As a result, the modulus and strength of the yarn is less than that of the fibers. 


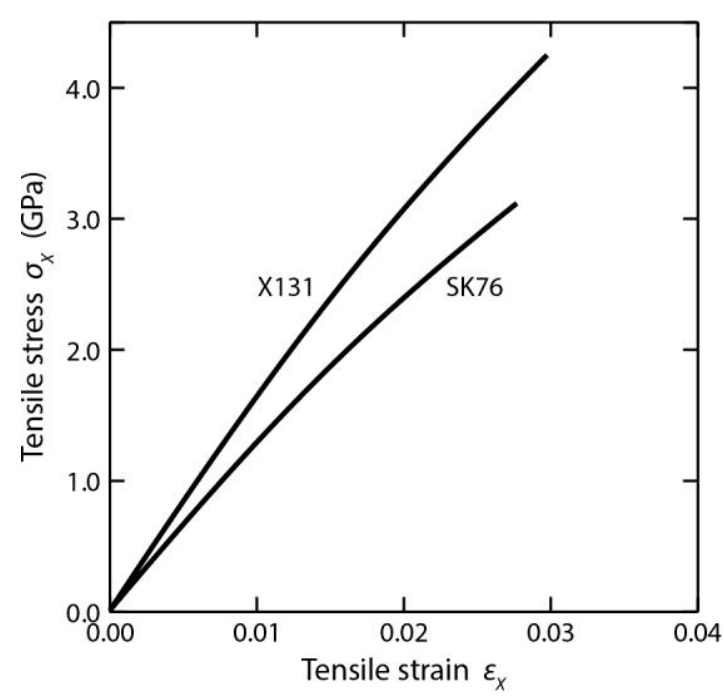

Figure 3.1. A typical response of Dyneema ${ }^{\circledR}$ SK76 and X131 yarns when pulled in tension at a nominal strain rate of $10^{-2} \mathrm{~s}^{-1}$.

\subsection{Laminate tensile response}

\subsubsection{Methodology}

Tensile testing of UHMWPE laminates commonly employs non-standardized coupon geometries and gripping methodologies to overcome significant specimen slip at the grips $[1,10,11]$. Here a test methodology proposed by Russell et al. [1] was modified by using hydraulic grips and a hole free gripping area, Figure 3.2. The coupons were milled from an eight ply, $\left[0^{\circ} / 90^{\circ}\right]_{4}$ consolidated plate sandwiched between sacrificial pairs of aluminum plates. The outer $90^{\circ}$ ply, whose fibers were transverse to the sample longitudinal axis, were removed in the grip region to further reduce grip slip, and a pair of hydraulic grips then applied a $50 \mathrm{MPa}$ pressure to the gripped region of the coupon. An Instron model 4208 mechanical testing machine extended the sample at a nominal tensile strain rate of $10^{-3} \mathrm{~s}^{-1}$ in the gauge section of the test coupon, and a laser extensometer was used to measure the displacement of two reflective tags attached within the gauge section. 


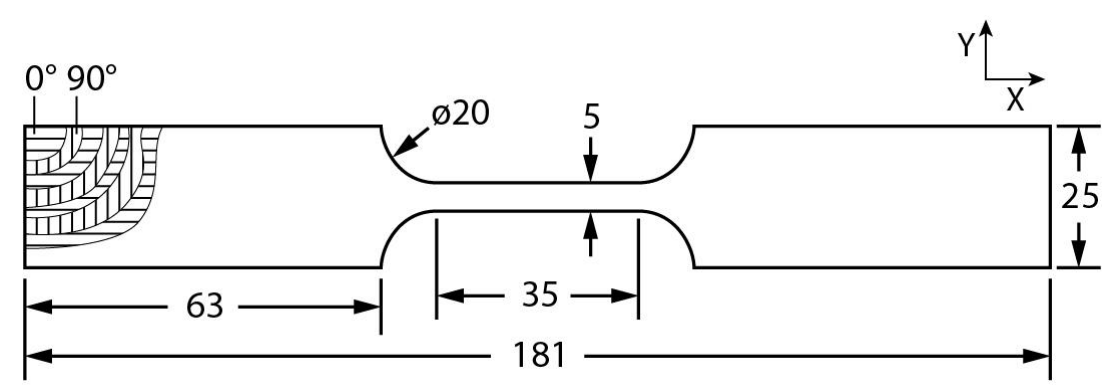

Figure 3.2. Cross-ply laminate sample design for measuring the tensile response. All dimensions are in millimeters.

\subsubsection{Results}

A typical tensile stress versus strain response for a HB26 laminate is shown Figure 3.3. It was approximately linear-elastic prior to the start of failure, which occurred in multiple discrete steps, reaching a peak strength of about $820 \mathrm{MPa}$. Given that the matrix contribution to the strength is negligible (given the low in-plane shear [2] and through-thickness, Section 3.3.2, shear strengths), laminate plate theory [12] predicts that the $\left[0^{\circ} / 90^{\circ}\right]$ laminate with $83 \%$ volume fraction fiber should have a strength of about 1.3 GPa based on the yarn measurements reported in Section 3.1.2. The reduction in strength from that expected for the laminate given the yarn strengths is thought to be a consequence of fiber waviness that developed in the laminate during fabrication: the wavy fibers then straighten out during the loading which gives rise to non-uniform loading of the constituent fibers of the composite. 


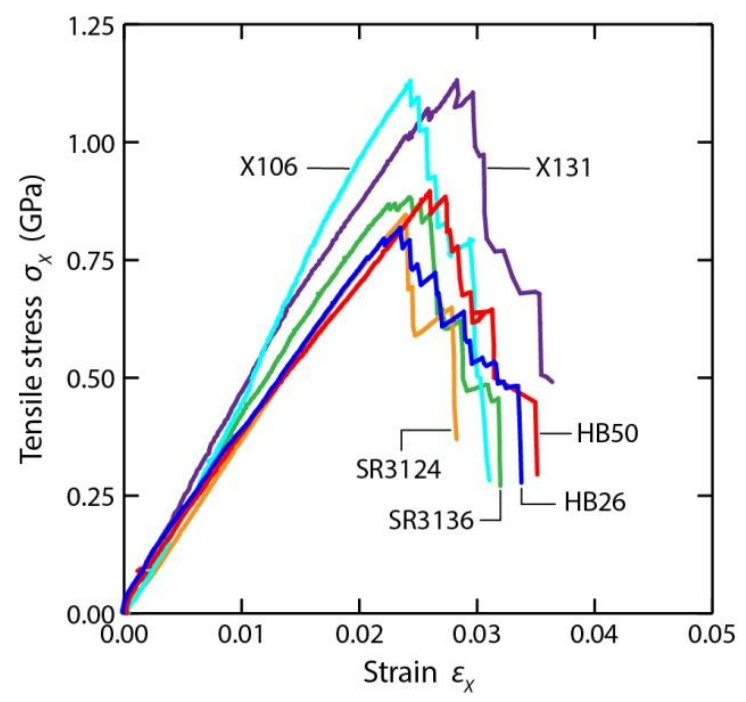

Figure 3.3. The measured laminate tensile response for each grade of material.

A typical tensile response for each of the other five material grades is also shown in Figure 3.3. The response is fiber dominated. The SK76 fiber based HB26 and HB50 laminates have similar strengths and responses and are both weaker than X106 and X131 laminate grades which used higher strength SK90 and X131 fibers, respectively. The Spectra Shield $^{\circledR}$ II grades SR3124 and SR316 also have similar responses to Dyneema ${ }^{\circledR}$ HB26. Table 2.2 tabulates the ply strength, which was calculated as twice the laminate peak strength, for each laminate.

\subsection{Inter-laminar shear}

\subsubsection{Methodology}

The inter-laminar shear strength, $\tau_{0}$, was measured using a double-notch specimen proposed by Liu et al. [4] and others [2,3,5], Figure 3.4(a). The sample design utilizes three notches (here introduced into the coupon during the tape lay-up stage so as to minimize coupon damage) to measure a through-the-thickness inter-laminar shear stress. 
Each $\left[0^{\circ} / 90^{\circ}\right]_{40}$ test coupon was $200 \mathrm{~mm}$ long by $w=20 \mathrm{~mm}$ wide, and had a gage length, $L_{g}=20 \mathrm{~mm}$. The test coupon was longitudinally extended at a displacement rate of $1 \mathrm{~mm} \mathrm{~min}^{-1}$, and a laser extensometer measured the displacement of two reflective tags adhered to either side of the coupon gage section. The inter-laminar shear stress, $\tau_{Z X}$, was defined as $\tau_{Z X}=F_{X} /\left(2 w L_{g}\right)$, where $F_{x}$ is the measured tensile load and the factor of two accounts for the shear loading occurring over two shear planes, as shown in Figure 3.4(b). The shear stress is presented as a function of Z-direction displacement between the two laser tags on either side of the gauge length (Figure 3.4(a)) as shear strain could not be determined from this experiment.

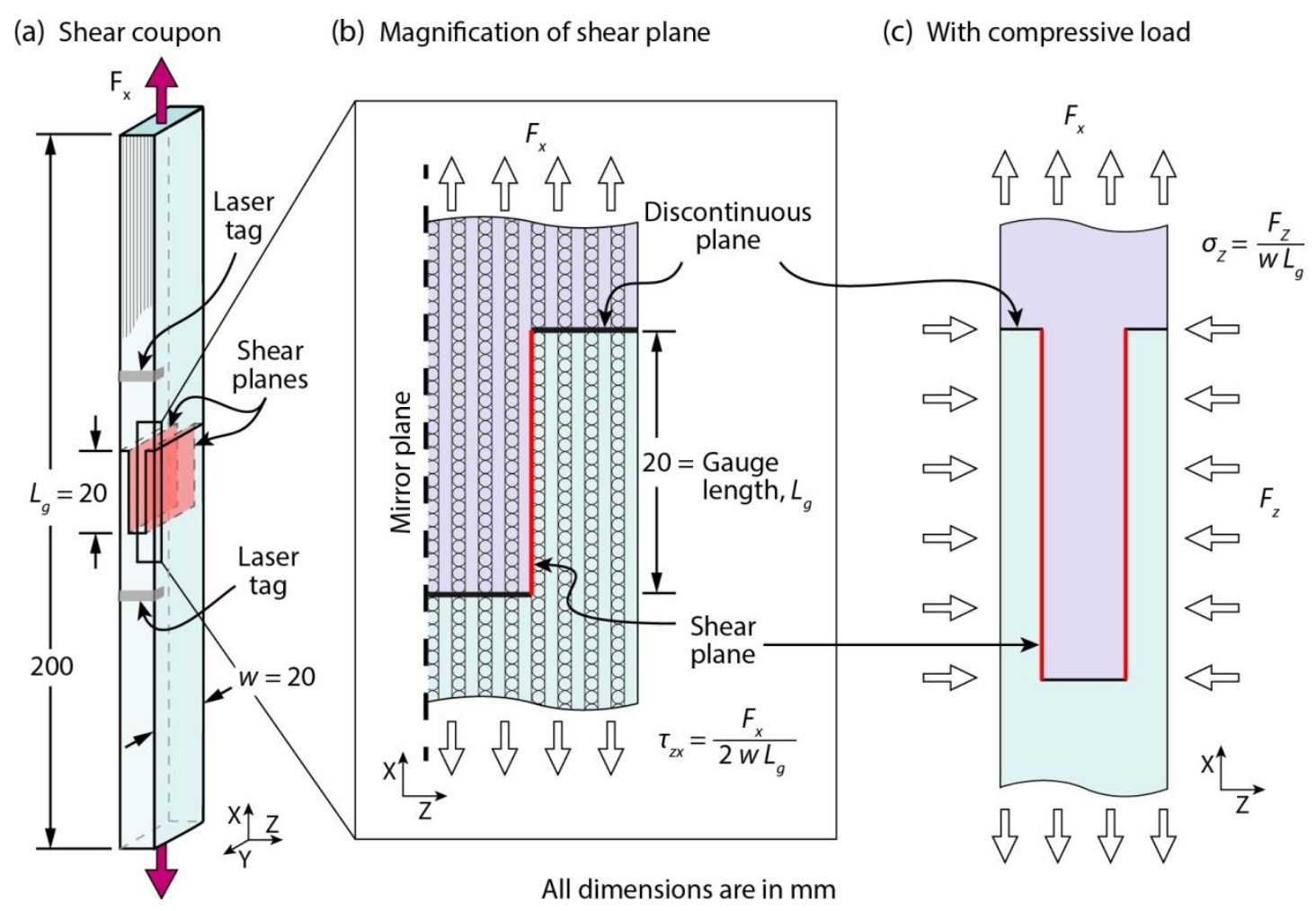

Figure 3.4. (a) Cross-ply laminate sample design for determining the interlaminar shear strength. (b) Magnified view of (a) identifying one of two shear planes. (c) Modified shear test where a out of plane compressive load is applied over the shear planes. 


\subsubsection{Results}

A representative inter-laminar shear stress verses displacement response for each material is shown in Figure 3.5. For HB26, the stress is observed to monotonically increase with displacement reaching a peak strength, $\tau_{0}=1.7 \mathrm{MPa}$. Continued displacement was accompanied by a rapid decrease in load towards a plateau strength near zero. Karthikeyan et al. [2] observed a similar response, and determined that at peak load shear localizes to an undetermined thin layer on each of the shear planes. A similar shear response was observed for the other five materials, Figure 3.5, and their average peak strengths, $\tau_{0}$, (from five repeated tests with different samples) are included in Table 2.2 (the standard deviation of these measurements was 5 to 10\%). The HB26 and SR3136 laminates embedded in the polyurethane based matrix had higher peak strengths than those that used the styrene based matrix. The Dyneema ${ }^{\circledR}$ laminates HB50, X106 and X131 utilized the same matrix, and had very similar strengths.

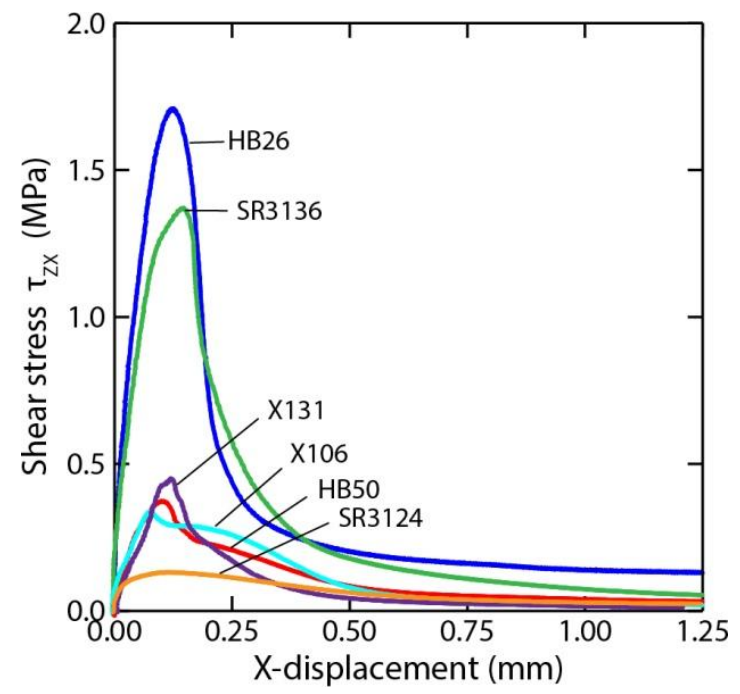

Figure 3.5. The inter-laminar shear stress, $\tau_{Z X}$, plotted as a function of displacement in the Xdirection for each material. 


\subsection{Shear strength dependence on compression}

This section presents a preliminary study of the dependence of the inter-laminar shear strength to out of plane compression for the HB26 laminates.

\subsubsection{Methodology}

A mechanism for applying a transverse compression to an inter-laminar shear strength test sample is shown in Figure 3.6. The external mechanism was designed to apply a uniform Z-oriented load, $F_{z}$, over the $w L_{g}$ area of the inter-laminar shear strength sample, Figure 3.4(c). The load, $F_{z}$, was measured by an Omega Engineering Inc. (Stamford, CT) model LC307-1K load cell with a $13.3 \mathrm{kN}$ capacity. The load was manually applied using a screw-driven ram to press together three 304 steel plates, Figure 3.6(b). The inter-laminar shear sample was sandwiched between the first two plates and the load cell was between the second and third plates. During the experiment, the sample was first loaded in through thickness compression, and then the sample was pulled in tension at a displacement rate of $1 \mathrm{~mm} \mathrm{~min}^{-1}$.
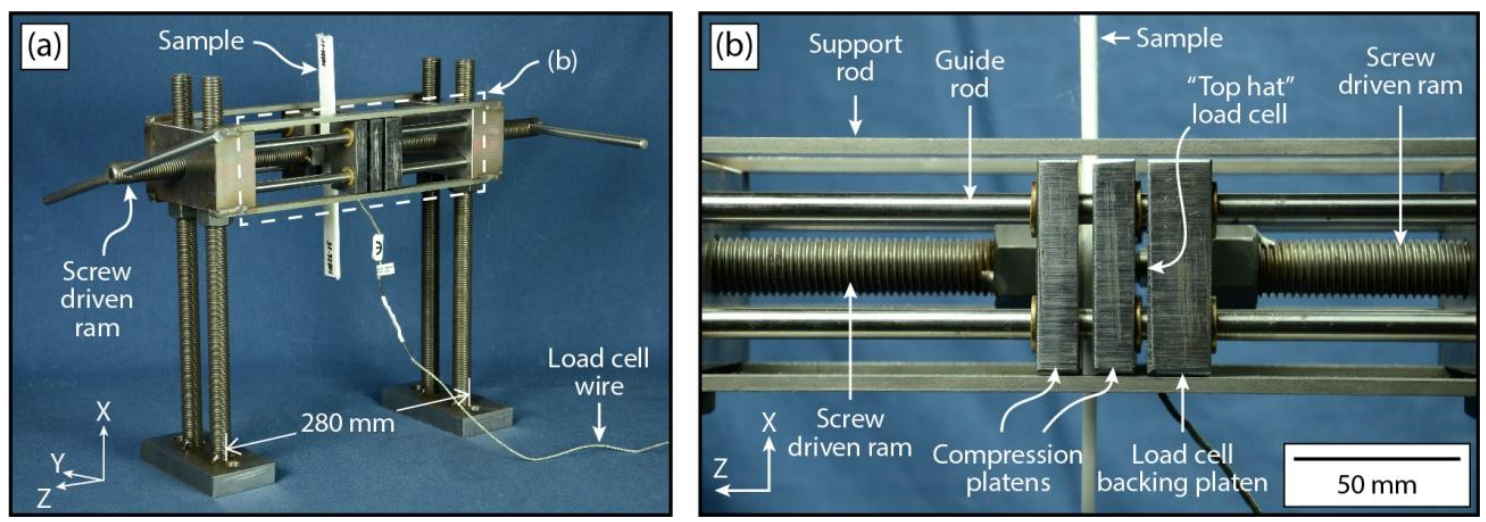

Figure 3.6. Load frame addition applies a compressive load, $\sigma_{z}$, over the sample gauge section, $w L_{g}$, while the sample is pulled in tension. (a) shows an oblique view, while the view in $(b)$ is normal to the XZ plane. 


\subsubsection{Results}

The inter-laminar shear stress as a function of Z-component displacement for HB26 under two compressive loadings, $\sigma_{Z}=7$ and $13 \mathrm{MPa}$, is shown in Figure 3.7(a). As before, the stress increased with displacement, reached a peak and was followed by a reduction in strength with further displacement. Figure 3.7(b) shows that the peak strength linearly increased with applied compressive stress. The inter-laminar shear strength almost doubled as the compressive stress, $\sigma_{Z}$, was increased from 0 to $13 \mathrm{MPa}$.
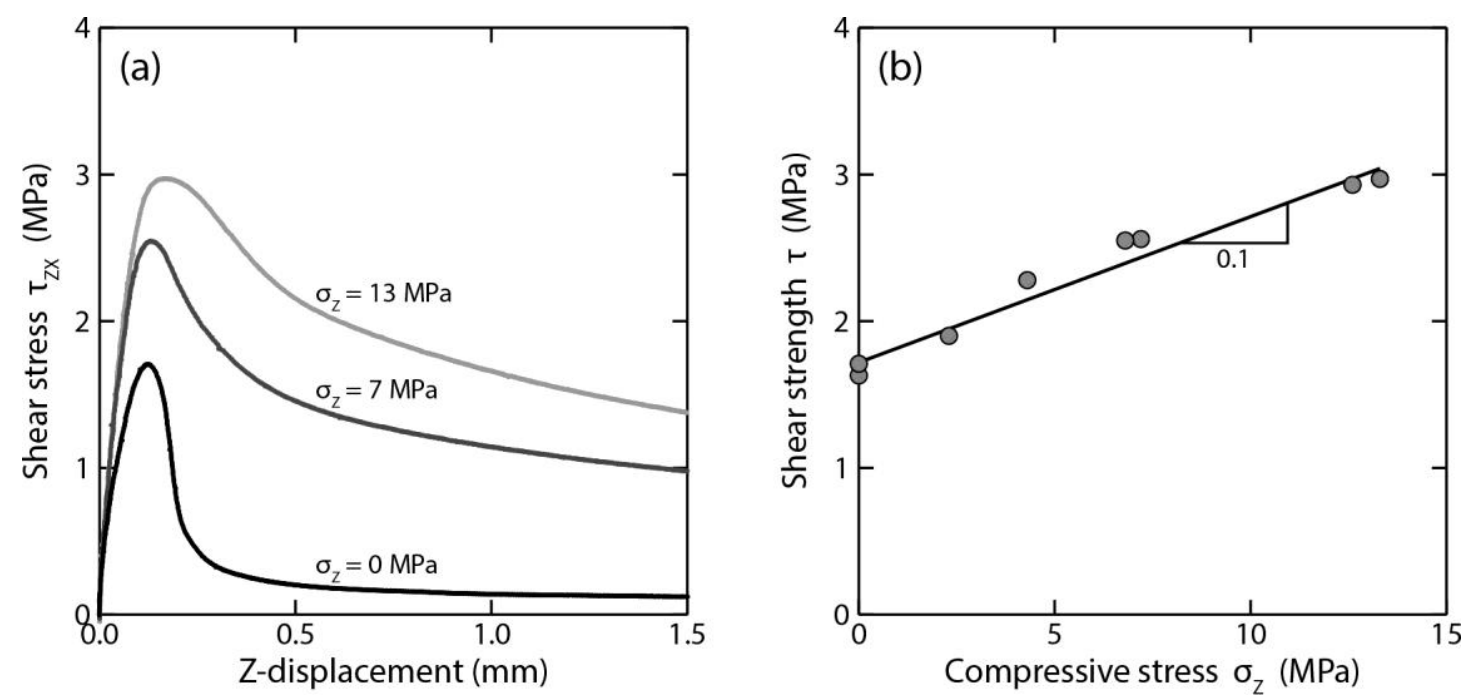

Figure 3.7. The influence of out of plane compression, $\sigma_{Z}$, on (a) the inter-laminar shear stress, $\tau_{Z X}$, plotted as a function of displacement and $(b)$ the shear strength, $\tau$, for HB26.

\subsection{Compression of unidirectional laminates}

\subsubsection{Methodology}

Unidirectional $\left[0^{\circ}\right]_{45}$ laminates were fabricated from $0^{\circ}$ ply HB26 pre-preg tape. Laminate consolidation followed the same procedure outlined in Section 2.3, except the pre-preg tape stack was heated and pressed in a pocket die to prevent lateral extrusion and concomitant ply thinning. Compression samples with in-plane dimensions of $L \times L$, 
were cut from the $2.8 \mathrm{~mm}$ thick laminated plate, Figure 3.8. Each sample was centered between two platens of A2 tool steel. The sample/platen set-up was then centered between the load frame platens of the screw driven testing machine equipped with a 50 $\mathrm{kN}$ load cell. The samples were compressed at a displacement rate of $0.6 \mathrm{~mm} \mathrm{~min}^{-1}$. The displacement was measured using a laser extensometer with laser tags attached to the sample platens.

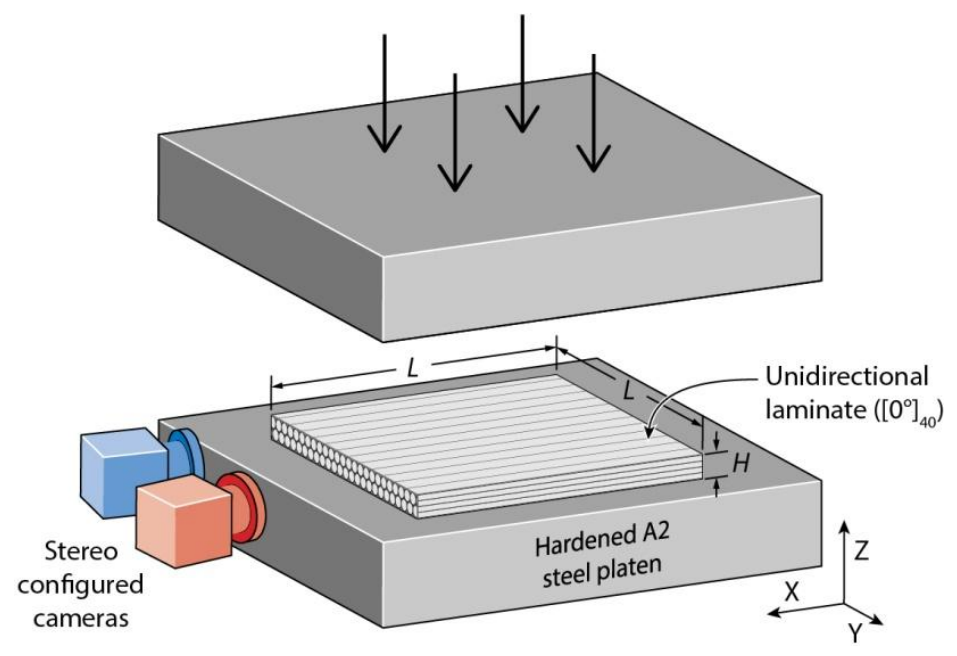

Figure 3.8. Schematic illustration of an L square unidirectional sample under uniform out of plane compression.

\subsubsection{Digital image correlation}

Poisson expansion due to the out of plane (Z-oriented) compressive loading was measured using a three-dimensional digital image correlation (DIC) system. The system tracks the displacement of a speckle pattern applied to a surface. Here the YZ surface (normal to the fiber direction) was first spray painted white, followed by the application of a stochastic pattern of 20-100 $\mu \mathrm{m}$ diameter black dots that covered approximately $50 \%$ of the surface area. A pair of 5 megapixel CCD cameras equipped with $50 \mathrm{~mm}$ lenses was positioned in a stereo configuration to capture the speckle pattern coated HB26 surface, Figure 3.8. A typical view captured by each camera is shown in Figure 3.9. Each black 
dot occupied approximately 50 pixels of a camera's field of view, and the cameras were synchronized to capture images at $0.25 \mathrm{~s}$ intervals.
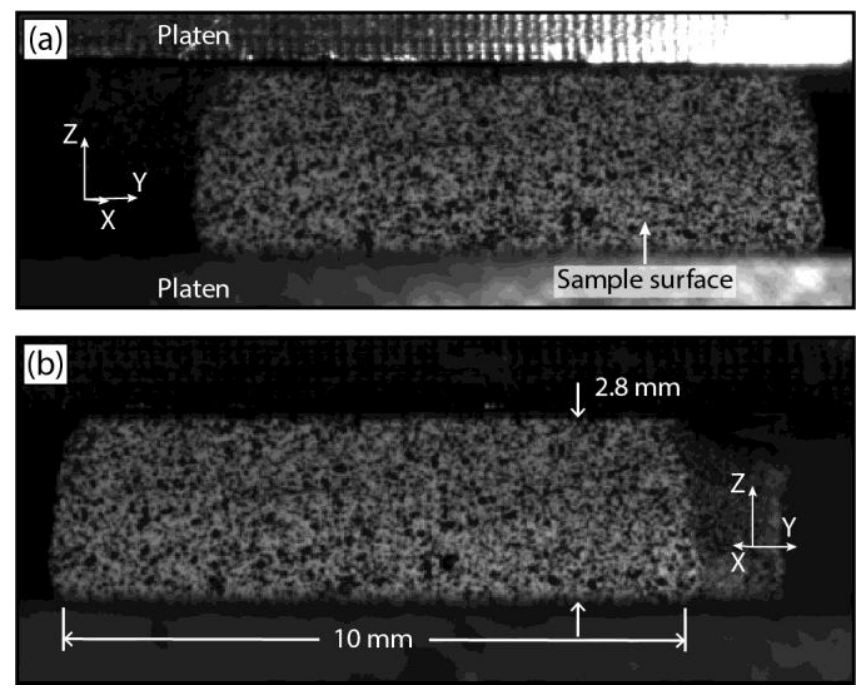

Figure 3.9. The field of views as seen from (a) the left and (b) the right cameras of the speckle coated YZ plane of an $L=10 \mathrm{~mm},\left[0^{\circ}\right]_{45}$, unidirectional $H B 26$ sample.

The DIC images were analyzed using Aramis v.6.3 (GOM mbH; Braunschweig, Germany) 3D image correlation software. The software first divided the left image from the stereo pair into interrogation areas (facets). Each facet was uniquely defined by the speckle pattern encompassed within its 25 pixel x 25 pixel area, and each linear raster of facets was spaced 15 pixels $(125 \mu \mathrm{m})$ apart. A calibration, using images taken of a NISTtraceable CQ $15 \mathrm{~mm}$ x $12 \mathrm{~mm}$ calibration panel (GOM mbH), was used to identify each facet in the right image and convert the facet location into the laboratory frame $(\mathrm{X}, \mathrm{Y}, \mathrm{Z})$ coordinate system. The locations of each facet were recorded for each image pair for the sequence of video images, and the software then calculated the full-field displacements and strains over the speckled surface as functions of time. Additional details can be found in references $[13,14]$. 


\subsubsection{Results}

The measured engineering out of plane stress, $\sigma_{Z}$, versus strain, $\varepsilon_{Z}$, responses for $L=9$ and $13 \mathrm{~mm},\left[0^{\circ}\right]_{45} \mathrm{HB} 26$ samples are shown in Figure 3.10(a). The responses for both sample sizes are similar: after an initial compliant response (believed to be due to voids as discussed in Chapter 5), the stress linearly increases with strain before reaching a local peak strength of approximately $20 \mathrm{MPa}$. After the peak, there was a slight reduction in stress followed by significant hardening with increasing strain. Unloading curves in the post-peak regime reveal minimal strain recovery which is indicative of significant permanent deformation. Photographs taken normal to the $\mathrm{YZ}$ sample plane (along the fiber axis) show the progressive deformation of an $L=13 \mathrm{~mm}$ sample, Figure 3.10 (b). Peak strength coincided with commencement of shear failure by the formation of shear bands. This was followed by significant plastic expansion in the Y-direction, while the Xdirection suffered negligible plastic deformation; compare Figure 3.10(c) and (d). The hardening behavior after the peak in strength is primarily due to this geometric effect, as the stress was calculated using the original sample area. Furthermore, given failure occurred by shear localization between fibers, the peak strength and hardening behavior are believed to be primarily determined by the resin, similar to the situation encountered during measurements of the inter-laminar shear strength in Section 3.3. 
(a)

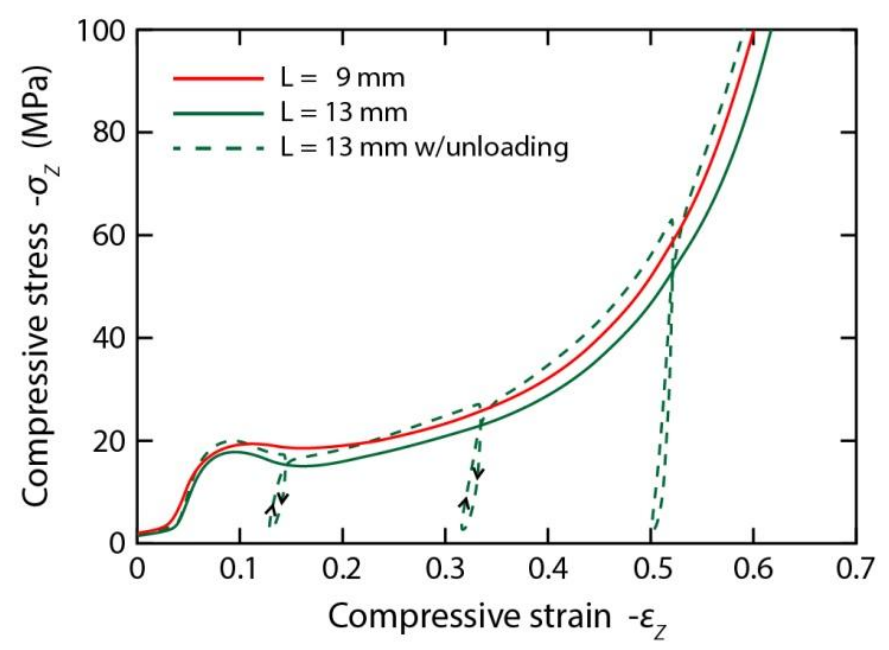

(b) In-situ planar view
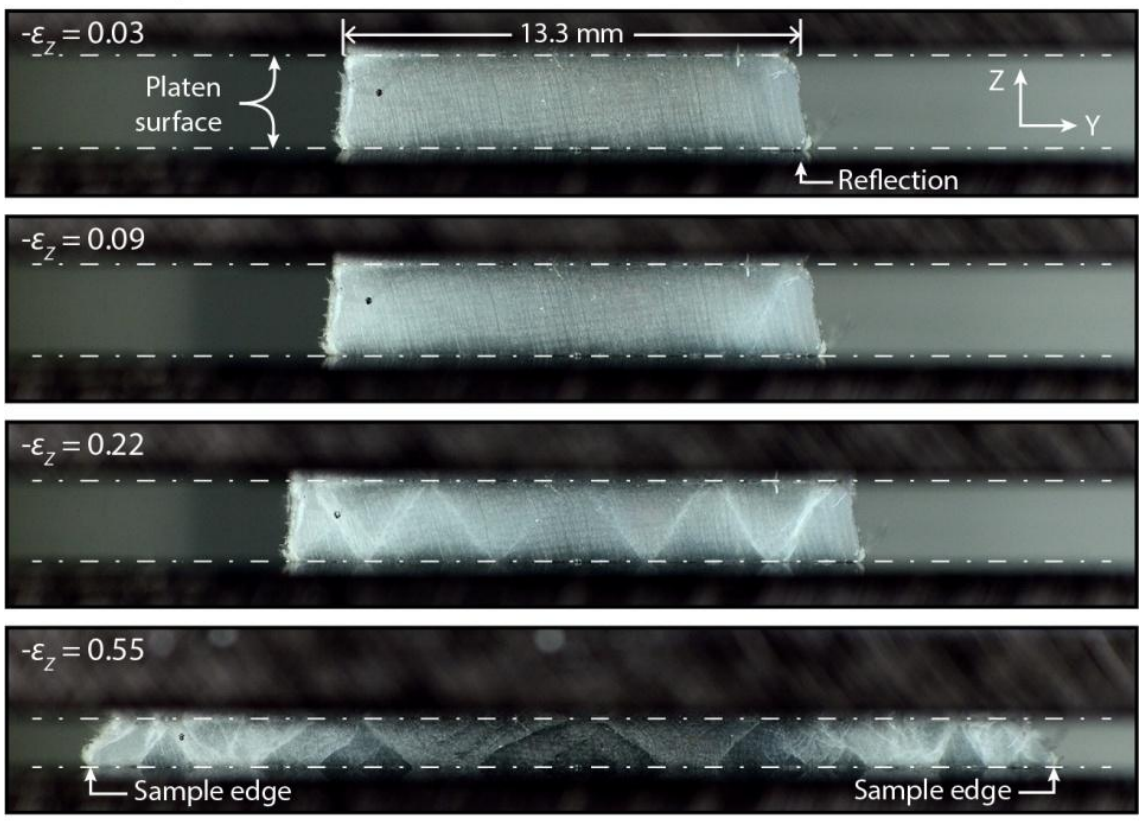

(c) Pre-test planar view

(d) Post-test planar view $\left(-\varepsilon_{z}=0.68\right)$
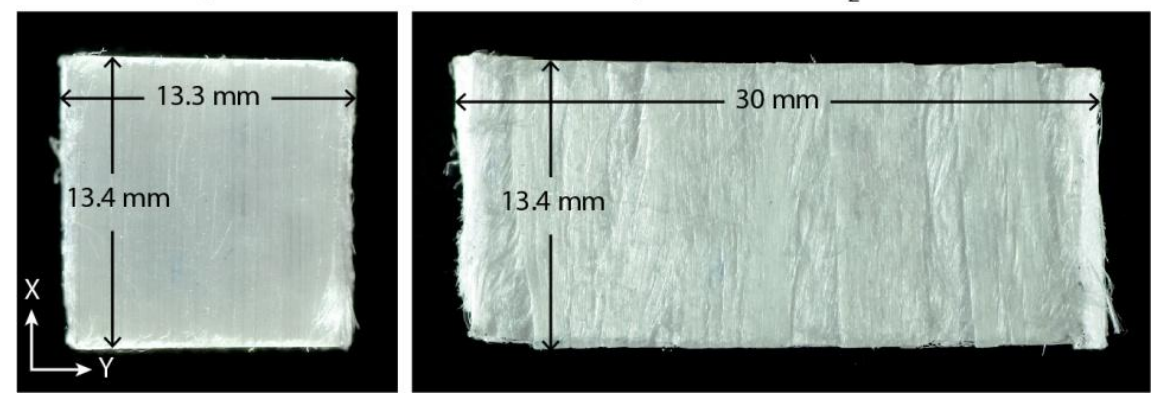

Figure 3.10. (a) The measured nominal stress versus strain of $\left[0^{\circ}\right]_{45}$, HB26 compression samples. (b) An image sequence showing the deformation of the $L=13 \mathrm{~mm}$ sample. The view is normal to the YZ plane. Images of the $L=13 \mathrm{~mm}$ sample, normal to the $X Y$ plane, are shown (c) before and (d) after testing. 
A series of unloading curves were recorded in the regime prior to shear failure, Figure 3.11(a) (for an $L=10 \mathrm{~mm}$ sample). The sample was observed to have inelastically deformed in this regime. Figure 3.11(b) plots the in-plane Y-component strain, $\varepsilon_{Y}$, determined from DIC measurements as a function of the out of plane (loading axis) strain, $\varepsilon_{Z}$. Note that both strain components were measured transverse to the fiber direction. Therefore an approximate measure of the ply's (elastic) transverse Poisson's ratio can be obtained from the slope $-\Delta \varepsilon_{Y} / \Delta \varepsilon_{Z}$ of the unload-reload portion of the curve. This slope is in the range of $0.4-0.5$, and subsequent models will use a transverse Poisson's ratio $v_{23}$ or $v_{Y Z}=0.5$. Experiments performed in the regime after shear failure show the ratio of strains increased to a value of 1 or greater. This value is expected to depend greatly upon sample geometry in this plastic flow dominated regime.
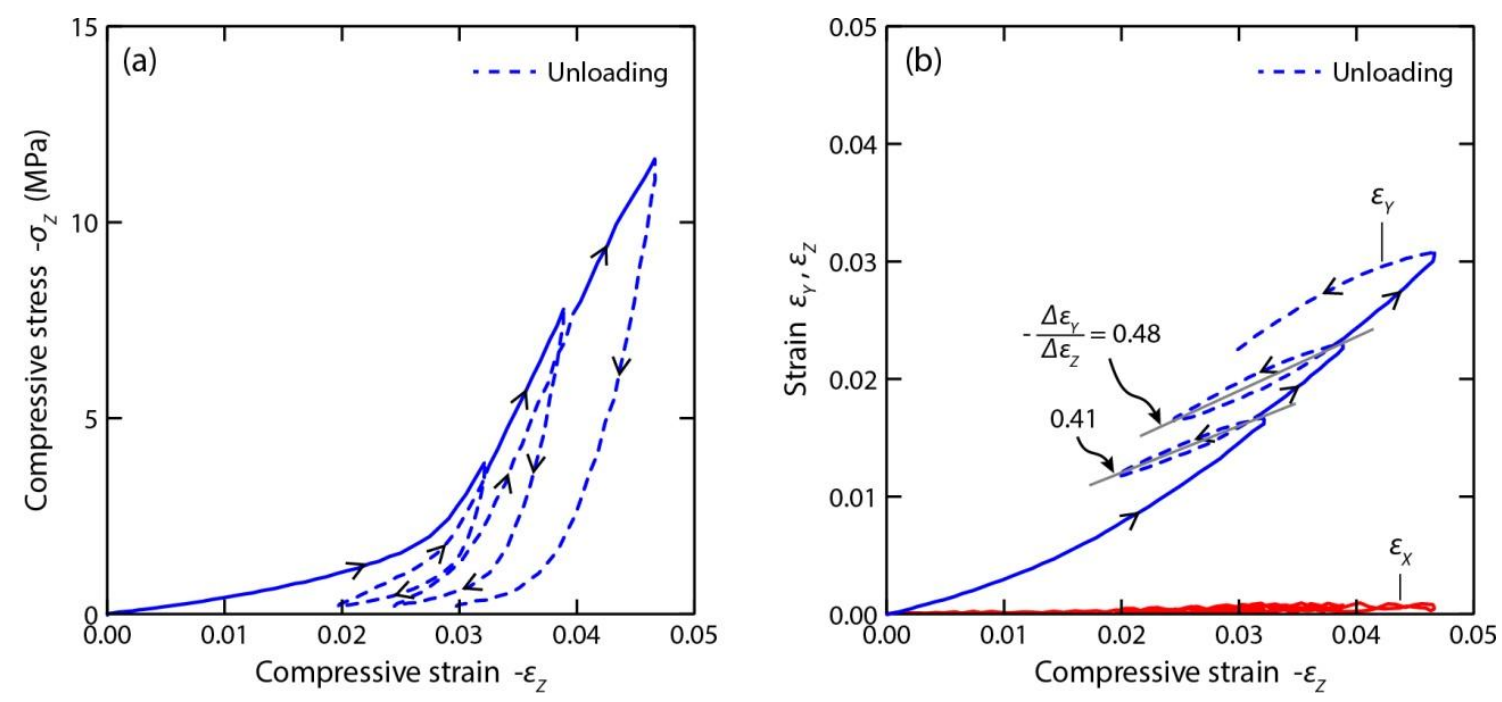

Figure 3.11. The compressive load-unload response of an $\mathrm{L}=10 \mathrm{~mm},\left[0^{\circ}\right]_{45}, \mathrm{HB} 26$ laminate in the regime prior to shear failure. Plotted as functions of the compressive strain, $\varepsilon_{Z}$ are $(a)$ the compressive stress, $\sigma_{Z}$ and $(b)$ the transverse strains, $\varepsilon_{Y}$ and $\varepsilon_{X}$.

Also plotted in Figure 3.11(b) is the strain in the fiber direction, $\varepsilon_{X}$. This was estimated by dividing the DIC recorded displacement in the X-direction by half of the 
sample length $L$ in the X-direction. The factor of $1 / 2$ accounts for an expected equal, but opposite, displacement of the opposing YZ sample face. While unable to account for rigid body motion, this method was preferred over measuring the surface displacement of the $\mathrm{XZ}$ sample plane because of questionable adhesion of the outer fibers to the bulk sample. The strain $\varepsilon_{X}$ is observed to negligibly change with the out of plane strain, $\varepsilon_{Z}$. Subsequent models will approximate a unidirectional ply's longitudinal Poisson's ratio (in the fiber direction) as $v_{13}$ or $v_{X Z}=0$.

\subsection{Conclusions}

This chapter has provided measurements of the mechanical properties that will be used in subsequent chapters to analyze the response of the laminates studied here. The laminates were highly anisotropic: ply tensile strengths in the fiber direction were 3 to 4 orders of magnitude greater than the inter-laminar shear strength. The tensile strength was primarily controlled by the fiber strength, while the shear strength was a matrix dominated property. A preliminary study of the compressive stress dependence of the inter-laminar shear strength (in the low compression stress regime) showed it had a linear out of plane compression pressure dependency. This behavior is believed to be active during the out of plane compression of a cross-ply laminate (reviewed in detail in Chapter 5). The out of plane compression of the unidirectional laminate showed the laminate failed by shear localization along planes of maximum shear which was followed by extensive plastic (matrix) deformation. Poisson expansion in the fiber direction was determined to be negligible, while pronounced expansion was observed transverse to the fibers. This behavior will be contrasted to the compression response of a cross-ply 
laminate, where the anisotropic Poisson response activates the indirect tension mechanism (Chapter 5).

\subsection{References}

[1] Russell BP, Karthikeyan K, Deshpande VS, Fleck NA. The high strain rate response of Ultra High Molecular-weight Polyethylene: From fibre to laminate. Int J Impact Eng 2013;60:1-9.

[2] Karthikeyan K, Russell BP, Fleck NA, O'Masta MR, Wadley HNG, Deshpande VS. The soft impact response of composite laminate beams. Int J Impact Eng 2013;60:24-36.

[3] Karthikeyan K, Russell BP, Fleck NA, Wadley HNG, Deshpande VS. The effect of shear strength on the ballistic response of laminated composite plates. Eur J Mech ASolids 2013;42:35-53.

[4] Liu G, Thouless MD, Deshpande VS, Fleck NA. Collapse of a composite beam made from ultra high molecular-weight polyethylene fibres. J Mech Phys Solids 2014;63:320-35.

[5] Attwood JP, Khaderi S, Karthikeyan K, Fleck NA, O'Masta MR, Wadley HNG, et al. The out-of-plane compressive response of Dyneema composites. J Mech Phys Solids 2014:[Submitted for publication].

[6] Ward IM, Sweeney J. An Introduction to the Mechanical Properties of Solid Polymers. 2nd ed. Hoboken, NJ: John Wiley \& Sons, Inc.; 2004.

[7] Kromm FX, Lorriot T, Coutand B, Harry R, Quenisset JM. Tensile and creep properties of ultra high molecular weight PE fibres. Polym Test 2003;22:463-70.

[8] Wang J, Smith Jr. KJ. The breaking strength of ultra-high molecular weight polyethylene fibers. Polymer 1999;40:7261-74.

[9] Govaert LE, Peijs T. Tensile strength and work of fracture of oriented polyethylene fibre. Polymer 1995;36:4425-31.

[10] Czechowski L, Jankowski J, Kubiak T. Experimental Tests of a Property of Composite Material Assigned for Ballistic Products. Fibres Text East Eur 2012;3(92):61-6.

[11] Levi-Sasson A, meshi I, Mustacchi S, Amarilio I, Benes D, Favorsky V, et al. Experimental determination of linear and nonlinear mechanical properties of laminated soft composite material system. Compos Part B Eng 2014;57:96-104.

[12] Herakovich CT. Mechanics of Fibrous Composites. New York: John Wiley \& Sons, Inc.; 1998. 
[13] Sutton MA. Computer Vision-Based, Noncontacting Deformation Measurements in Mechanics: A Generational Transformation. Appl Mech Rev 2013;65:050000050000.

[14] Aramis User Manual - Software. Braunschweig, Germany: GOM mbH; 2013. 


\section{Chapter 4. Mechanisms of projectile penetration in HB26 encapsulated aluminum structures ${ }^{2}$}

The focus of this study is to elucidate the different mechanisms of ballistic impact deformation and failure of UHMWPE fiber-reinforced polymer matrix composites in model systems designed to reveal the fundamental micromechanisms. This was approached by experimentally observing the penetration of four model systems impacted by a $12.7 \mathrm{~mm}$ diameter spherical steel projectile. The four model targets were: (i) a bare aluminum plate; (ii) the same plate fully encased in a $5.9 \mathrm{~mm}$ thick casing of Dyneema ${ }^{\circledR}$; (iii) the fully encased plate with a portion of the Dyneema ${ }^{\circledR}$ removed from the front face so that the projectile directly impacted the Al plate; and (iv) the fully encased plate with a portion of the Dyneema ${ }^{\circledR}$ removed from the rear face so that the projectile could exit the $\mathrm{Al}$ plate without again interacting with the Dyneema ${ }^{\circledR}$. A combination of synchronized high speed photography with three cameras, together with post-test examination of the targets via X-ray tomography and optical microscopy was used to elucidate the

\footnotetext{
${ }^{2}$ A paper [1] assembled from the research in this chapter is in press with the International Journal of Impact Engineering.
} 
deformation and perforation mechanisms. The measurements show that the ballistic resistance of these targets increases in the order: bare Al plate, rear face cutout target, fully encased target and front face cutout target. These observations are explained based on the following key findings: (a) the ballistic performance of Dyneema ${ }^{\circledR}$ plates supported on a foundation is inferior to Dyneema ${ }^{\circledR}$ plates supported around their edges; (b) the apparent ballistic resistance of Dyneema ${ }^{\circledR}$ plates increases if the plates are given an initial velocity prior to the impact by the projectile thereby reducing the relative velocity between the Dyneema ${ }^{\circledR}$ plate and projectile; and (c) when the projectile is fragmented prior to impact, the spatially and temporally distributed loading enhances the ballistic resistance of the Dyneema ${ }^{\circledR}$.

The outline of the chapter is as follows. First we describe the four different model systems and the materials and fabrication methods. Next, the response of the four different systems to impact by a hard steel sphere is described and compared. Finally, these observations are assembled to elucidate the different perforation mechanisms of fiber-reinforced plastic matrix composites.

\subsection{Materials and sample fabrication}

The dynamic response of Dyneema ${ }^{\circledR}$ HB26 was probed by utilizing variations in the penetration mechanism of an aluminum plate when impacted by a hardened (low ductility) chrome steel sphere of diameter $12.7 \mathrm{~mm}$ at various impact velocities. The baseline target investigated in this study comprises a $6061 \mathrm{~T} 6$ aluminum alloy plate wrapped in grade HB26 Dyneema ${ }^{\circledR}$ as sketched in Figure 4.1(a). This is the same Dyneema $^{\circledR}$ grade used by Karthikeyan et al. [2] in their edge clamped plate impact study, 
and this study uses a similar thickness laminate to allow for appropriate comparisons. However, unlike in [2] the wrapping of the Dyneema ${ }^{\circledR}$ employed here allowed us to avoid using bolts for attaching the Dyneema ${ }^{\circledR}$ plates. Three additional variants of the baseline targets are also investigated within this study in order to gain further insights into the deformation and penetration mechanisms. The four types of targets were:

(i) The baseline fully encased target: this target comprised a $31.6 \mathrm{~mm}$ thick $6061 \mathrm{~T} 6$ aluminum alloy plate wrapped in Dyneema ${ }^{\circledR}$ HB26 cross-ply composite (Figure 4.1(a)).

(ii) The bare Al plate: a $31.6 \mathrm{~mm}$ thick $6061 \mathrm{~T} 6$ aluminum alloy plate used to characterize the ejecta impacting the rear laminate of the front face cutout target (Figure 4.1(b)). A $63.5 \mathrm{~mm}$ thick $6061 \mathrm{~T} 6$ aluminum alloy plate was additionally characterized to understand the projectile/target interaction during high impact velocity penetration.

(iii) The front face cutout target: a $30 \times 30 \mathrm{~mm}$ central section was cut out from the impacted face of the baseline target to understand the role of the Dyneema $^{\circledR}$ on the impacted face (Figure 4.1(c)).

(iv) The rear face cutout target: an $80 \times 80 \mathrm{~mm}$ central section was cut out from the distal face of the baseline target to understand the role of the Dyneema ${ }^{\circledR}$ on the rear, or non-impacted, face (Figure 4.1(d)). 

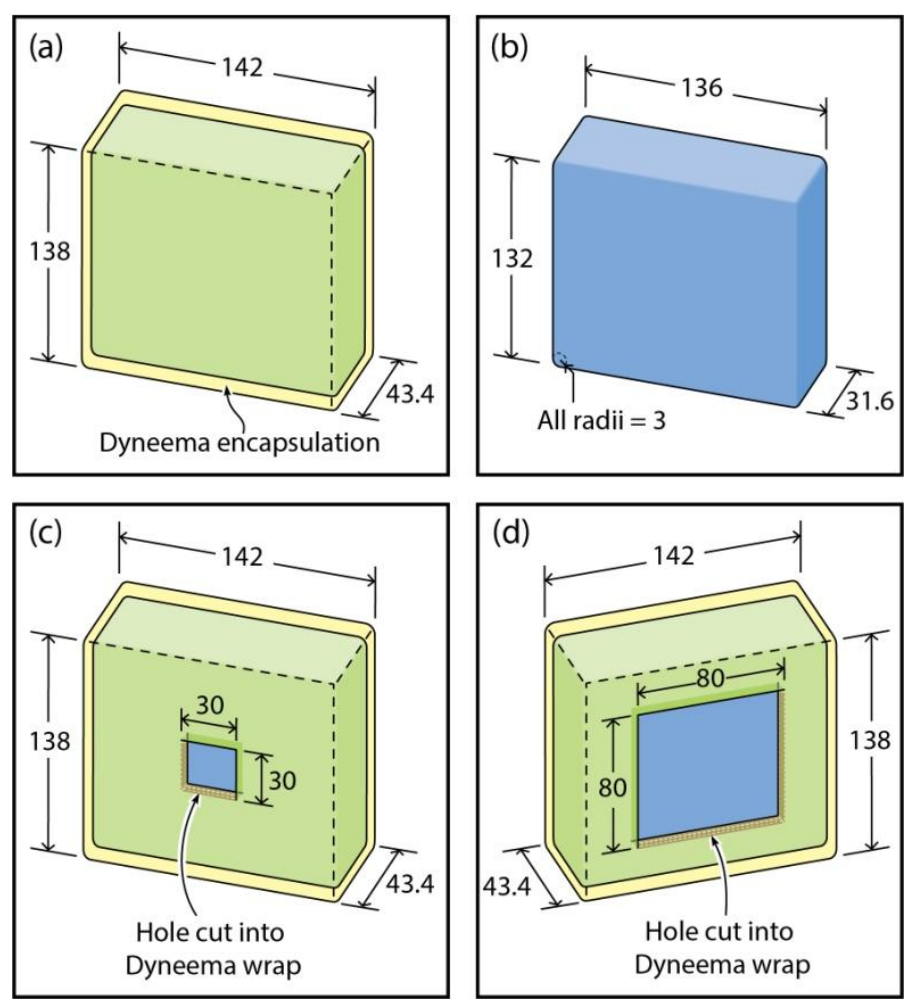

Figure 4.1. Sketches of the four model targets employed in this study. (a) The baseline target with the Al plate fully encased by Dyneema ${ }^{\circledR},(b)$ the bare Al plate, (c) the front face cutout target and (d) the rear face cutout target. All dimensions are shown in $\mathrm{mm}$.

These four target designs enable us to (i) probe the behavior of both an edge supported Dyneema ${ }^{\circledR}$ plate and a Dyneema ${ }^{\circledR}$ plate supported on a foundation when impacted by a spherical projectile, and (ii) investigate the behavior of an edge supported Dyneema ${ }^{\circledR}$ plate impacted by a fragmented projectile. We proceed to first describe the materials used in the study and then describe the manufacturing processes and the geometrical details. 


\subsubsection{Materials}

\subsubsection{Dyneema ${ }^{\circledR} H B 26$}

The HB26 grade composite comprises Dyneema ${ }^{\circledR}$ SK76 fibers in a polyurethane matrix and was received as a $\left[0^{\circ} / 90^{\circ}\right]_{2}$ pre-preg tape with a fiber fraction of $83 \mathrm{wt} . \%$. Please refer to Section 2.2 for additional details.

\subsubsection{Aluminum plate}

In all the Dyneema ${ }^{\circledR}$ wrapped targets used a $31.6 \mathrm{~mm}$ thick aluminum 6061 alloy plate solutionized and aged to the T6 condition. Quasi-static tensile tests were conducted on the 6061 plate material in the T6 condition. A typical tensile response at an initial nominal strain rate of $10^{-3} \mathrm{~s}^{-1}$ is shown in Figure 4.2. The measured Young's modulus and yield strength were $67 \mathrm{GPa}$ and $305 \mathrm{MPa}$, respectively, and the alloy had an ultimate tensile strength of $350 \mathrm{MPa}$ at a tensile failure strain of $14 \%$. Readers are referred to [3] for the dynamic behavior of this alloy, including a Johnson Cook model fit of its mechanical behavior.

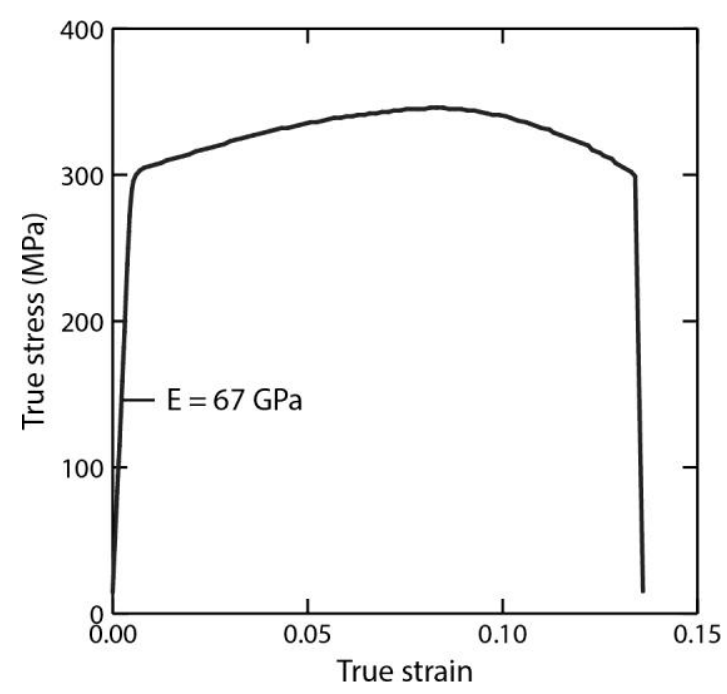

Figure 4.2. A typical true stress - strain uniaxial tensile of the 6061-T6 aluminum target material. 


\subsubsection{Projectile}

The projectile was a $12.7 \mathrm{~mm}$ diameter hardened 52100 chrome steel sphere, with a mass of $8.4 \mathrm{~g}$ (CCR Products, West Hartford, CT). Quasi-static compression tests were conducted on $6.35 \mathrm{~mm}$ diameter by $9.53 \mathrm{~mm}$ tall right circular cylinders cut from the spheres by wire electrical discharge machining (EDM). A typical measured response at an initial nominal strain rate of $10^{-4} \mathrm{~s}^{-1}$ is shown in Figure 4.3. The measured Young's modulus, yield strength and compressive strength were $205 \mathrm{GPa}, 2.4 \mathrm{GPa}$ and $3.4 \mathrm{GPa}$, respectively, and its measured hardness was $64 \mathrm{HRC}(7.6 \mathrm{GPa})$.

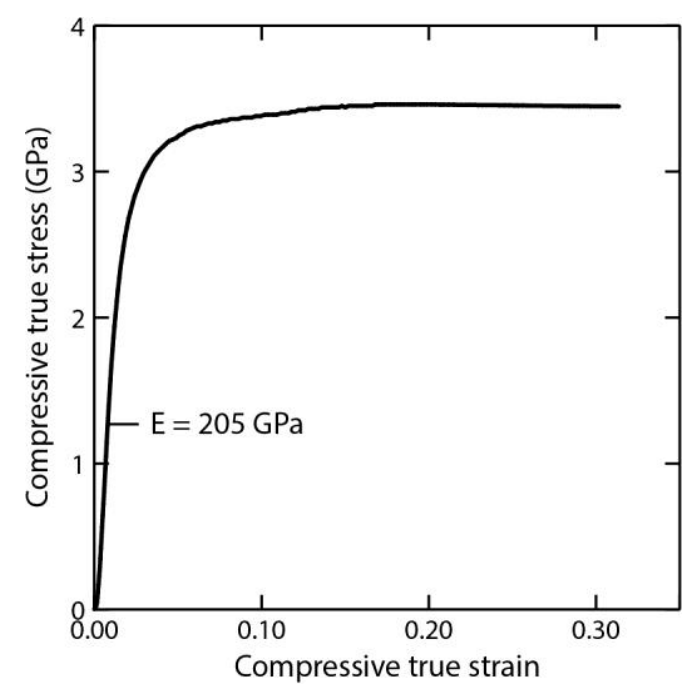

Figure 4.3. A typical true stress - strain response when compressing a right circular cylinder cut from the 52100 spherical projectile.

\subsubsection{Sample fabrication}

The three types of Dyneema ${ }^{\circledR}$ encapsulated samples are sketched in Figure 4.1. Here the manufacturing process is briefly described. All three target types first involved the manufacture of the baseline target as follows: 
Step I: The $136 \mathrm{~mm}$ x $132 \mathrm{~mm}$ x $31.6 \mathrm{~mm}$ thick rectangular Al plate had its edges chamfered to a radius of $3 \mathrm{~mm}$ to reduce stress concentrations on the Dyneema ${ }^{\circledR}$ wrapping (Figure 4.4(a)).
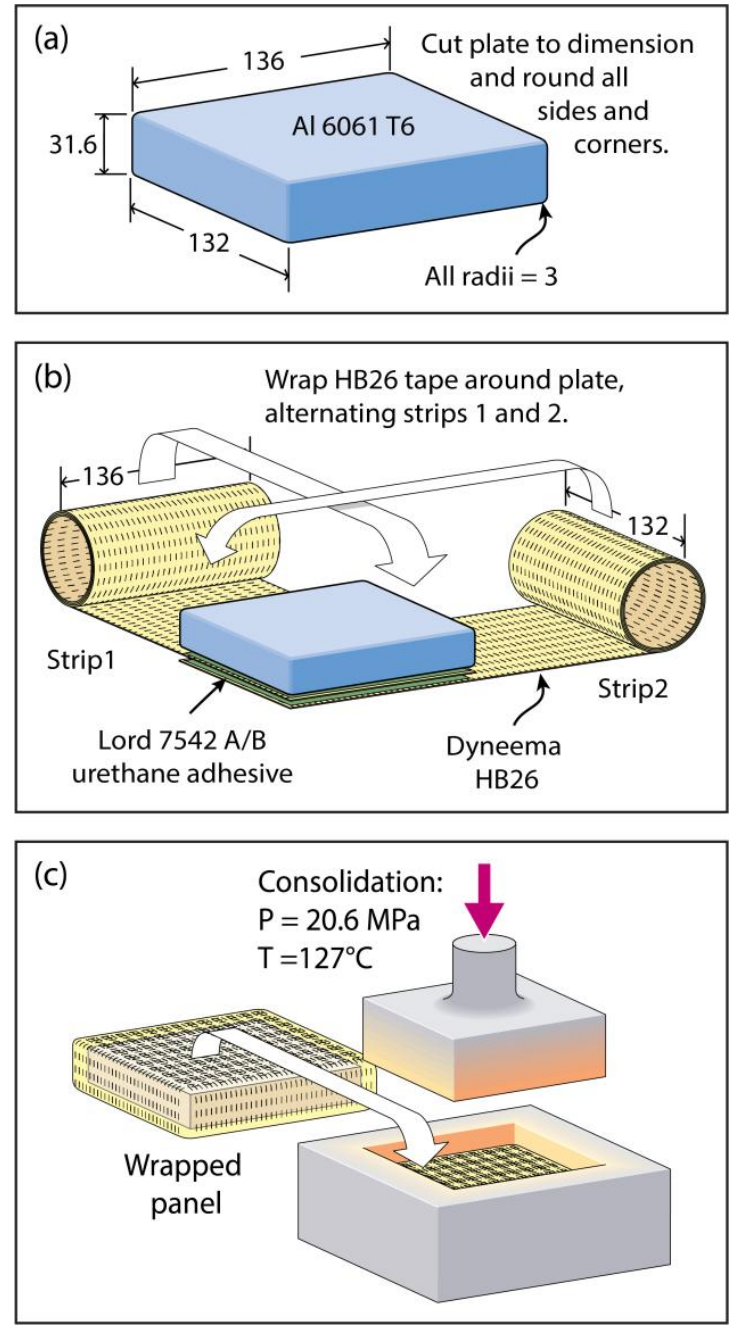

Figure 4.4. Fabrication of the Dyneema ${ }^{\circledR}$ encased aluminum panels using two strips of HB26 Dyneema ${ }^{\circledR}$ pre-preg tape. All dimensions are given in $\mathrm{mm}$.

Step II: Two strips of the HB26 pre-preg tape, one $136 \mathrm{~mm}$ wide (strip 1) and the other $132 \mathrm{~mm}$ wide (strip 2), were cut in the $0^{\circ} / 90^{\circ}$ fiber orientation to a length of 4 meters. The surface of strip 1 was bonded with a Lord grade 7542 A/B urethane adhesive (Lord Corp., Cary, NC) to the rear surface of the aluminum plate, and strip 2 was 
adhesively bonded to the surface of Strip 1 , but rotated $90^{\circ}$ to the wrapping direction of Strip 1; see Figure 4.4(b). The fibers in the outer ply of each strip were oriented parallel to the sides of the rectangular aluminum plate. Each strip was then alternatively wrapped around the aluminum plate eleven times without applying significant tension to the tapes $^{3}$. Referencing the outer ply of Strip 1 as the $0^{\circ}$ orientation, the lay-up from the aluminum surface on both the $136 \mathrm{~mm} \times 132 \mathrm{~mm}$ faces was $\left[\left(90^{\circ} / 0^{\circ}\right)_{2} /\left(0^{\circ} / 90^{\circ}\right)_{2}\right]_{11}$ with a thickness of $5.9 \mathrm{~mm}$. The lay-up on the four smaller sides was either $\left[90^{\circ} / 0^{\circ}\right]_{22}$ or $\left[0^{\circ} / 90^{\circ}\right]_{22}$ and thus only half the thickness of that on the $136 \mathrm{~mm} \times 132 \mathrm{~mm}$ faces.

Step III: The Dyneema ${ }^{\circledR}$ wrapped samples were consolidated in a hot press using a die, Figure 4.4(c). The procedure outlined in Section 2.3 was followed with the exception of a 20 minute hold time at temperature and pressure instead of 15 minutes. The final dimensions of the fully encased panel are indicated in Figure 4.1(a).

The front and rear face cutout targets as indicated in Figure 4.1 were prepared by first manufacturing the baseline targets as described above and then removing a central square of Dyneema ${ }^{\circledR}$ from one of the $136 \mathrm{~mm}$ x $132 \mathrm{~mm}$ faces using an abrasive wheel attached to a rotary cutting tool. The front and rear face cutout samples had $30 \mathrm{~mm}$ x 30 $\mathrm{mm}$ and $80 \mathrm{~mm}$ x $80 \mathrm{~mm}$ regions of Dyneema ${ }^{\circledR}$, respectively removed as shown in Figure 4.1. The aim was to cutout the minimum amount of Dyneema ${ }^{\circledR}$ wrapping so that the projectile did not make contact with the Dyneema ${ }^{\circledR}$ casing on the impacted face in the front face cutout targets. The rear face cutout had to be large enough to ensure that the

\footnotetext{
${ }^{3}$ The length of applied pre-preg material per strip was $\sim 5 \mathrm{~cm}$ longer than calculated for a perfectly tight wrap.
} 
wrapping on the rear face did not interact with the deformation of the rear surface of the aluminum plate or projectile/target ejecta.

A black ink square grid pattern of grid size $12.7 \mathrm{~mm}$ was applied to the (white) front and rear surfaces of all the Dyneema ${ }^{\circledR}$ encased samples. The grid was aligned with the $0^{\circ}$ and $90^{\circ}$ fiber orientations and permitted dynamic tracking of Dyneema ${ }^{\circledR}$ displacements via high speed photography during the impact events.

\subsection{Impact test protocol}

High speed ballistic impact tests were conducted by firing the $12.7 \mathrm{~mm}$ diameter hardened steel spheres described in Section 4.1.1.3. The projectile impacted the targets normally and centrally at velocities in the range 110 to $3300 \mathrm{~m} \mathrm{~s}^{-1}$. The penetration response of targets at these high velocities was not sensitive to the edge griping conditions; the fixture sketched in Figure 4.5(a) was employed to hold the targets in position during the tests.
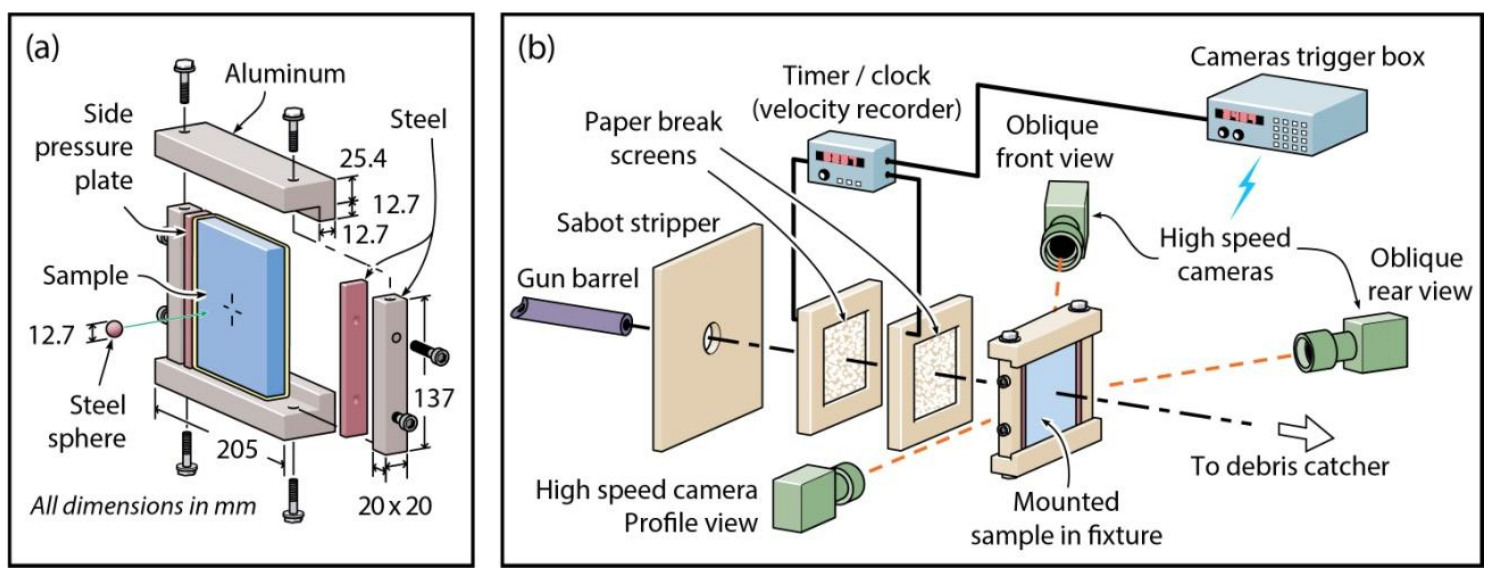

Figure 4.5. Schematic illustration of (a) the test fixture used to grip the targets and $(b)$ the dynamic testing facility at Chesapeake Testing (Belcamp, MD) used to perform the ballistic experiments. 
All impact tests with projectile impact velocities $V_{i} \leq 1700 \mathrm{~m} \mathrm{~s}^{-1}$ were performed at Chesapeake Testing (Belcamp, MD) using the setup sketched in Figure 4.5(b). Two paper break screens were used to measure the projectile impact velocities, and three v. 7.3 Phantom high speed cameras (Wyane, NJ) were used to obtain simultaneous high speed images of the profile, front and rear views of the sample. The images were recorded using $1 \mu$ s exposure durations at a $20 \mu$ s inter-frame interval. In case the projectile penetrated the target, the residual velocities $V_{r}$ were estimated from the images of the profile camera to an accuracy of $\pm 2.5 \%$. A debris catcher, consisting of compliant unconsolidated Dyneema ${ }^{\circledR}$ pre-preg tape, was placed behind the targets in order to arrest the projectile with minimal additional damage to the projectile. Recovered projectiles were analyzed for damage and fragmentation. A few select tests at impact velocities in excess of $1700 \mathrm{~m} \mathrm{~s}^{-1}$ were performed at the University of California, Santa Barbara using a light gas gun [4] with instrumentation and fixtures similar to those used at the Chesapeake testing facility.

After impact, most of the samples were water jet sectioned along a central plane to reveal internal damage. For any laminate that was partially perforated, the number of surviving plies below the projectile was counted. Some samples were also examined by high resolution X-ray computed tomography (XCT).

\subsection{Impact response of targets}

The measured impact velocity $V_{i}$ versus residual projectile/target ejecta (ejected material from distal side of target) velocity $V_{r}$ responses of all four target types investigated are plotted in Figure 4.6. In all cases the targets show a typical ballistic 
behavior wherein the projectile does not fully penetrate the target up to a critical velocity referred to as the ballistic limit $V_{b l}$, and $V_{r}=0$ for $V_{i}<V_{b l}$. Just above the ballistic limit the residual velocity of the projectile rises sharply and then $V_{r}$ increases more gradually with further increases in $V_{i}$. The ballistic limit $V_{b l}$ increases in the order: bare Al target, rear face cutout target, baseline fully encased target, and front face cutout target (with highest ballistic resistance). While we would have anticipated that the bare Al target would be the worst performing in terms of the ballistic limit, there are some non-intuitive observations from these measurements:

(i) The front face cutout target has as higher ballistic limit compared to the baseline fully encased target.

(ii) The rear face cutout target has a significantly lower ballistic limit compared to the fully encased target. In fact the ballistic limit of the rear face cutout target was only about $100 \mathrm{~m} \mathrm{~s}^{-1}$ higher that the bare Al target, and ejecta had a higher residual velocity than the bare aluminum plate for $V_{i}>1500 \mathrm{~m} \mathrm{~s}^{-1}$.

These observations suggest that the Dyneema ${ }^{\circledR}$ on the front and rear of the Al plate has profoundly different effects on the ballistic resistance of the targets. We proceed to first describe in detail the penetration responses of the four target types and then use these observations to explain the apparently anomalous behavior listed above and thereby gain insight into the ballistic penetration mechanics of Dyneema ${ }^{\circledR}$ composites. 


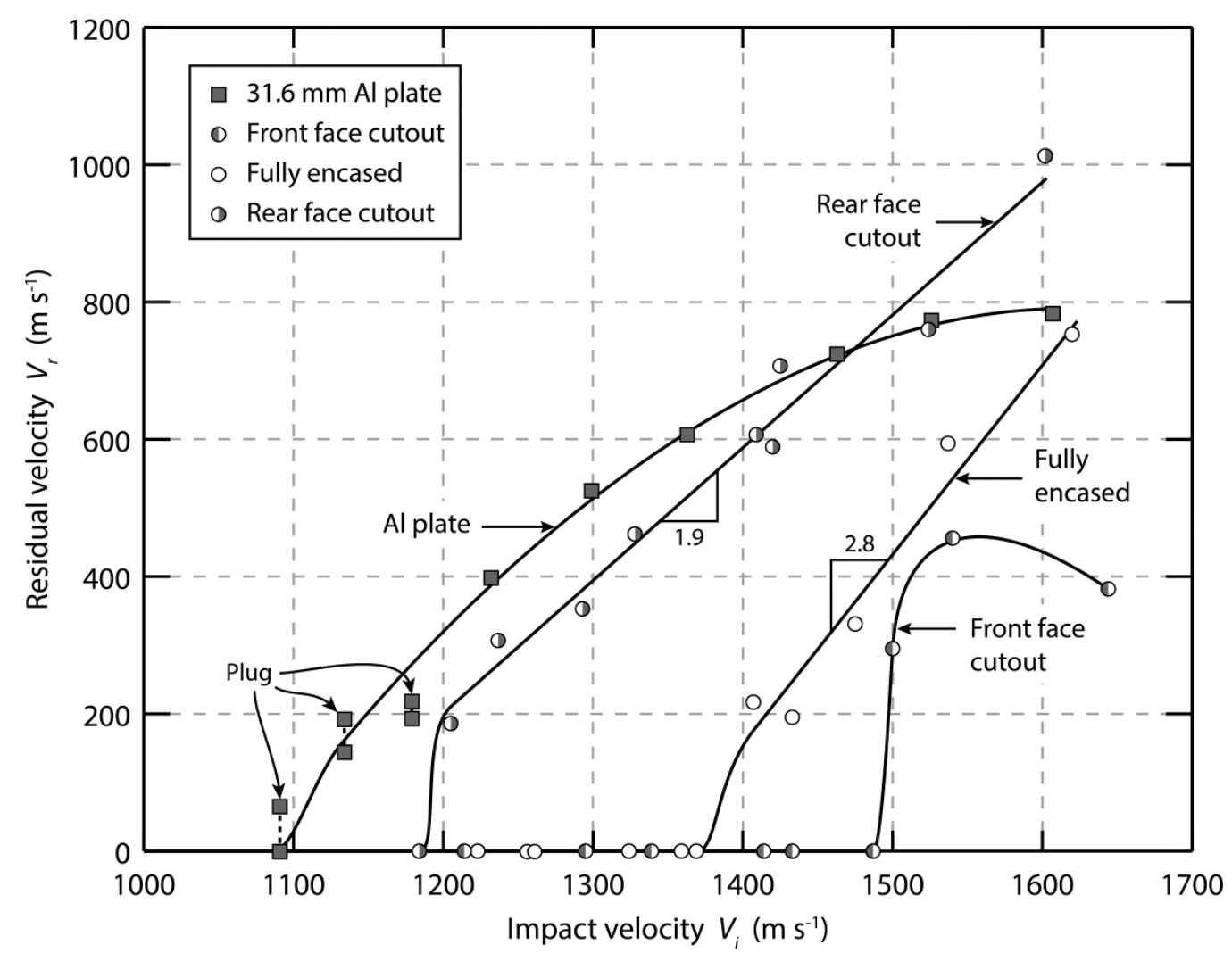

Figure 4.6. Summary of the measured residual (exit) velocity $V_{r}$ of the projectile/ejecta as a function of impact velocity $V_{i}$ for the four model targets.

\subsubsection{The bare aluminum plate}

The ballistic limit of the $31.6 \mathrm{~mm} \mathrm{Al}$ plate was significantly lower than the Dyneema $^{\circledR}$ encased targets. In order to characterize the deformation/fracture of the projectile and aluminum plate over the larger impact velocity range investigated for the encased targets, we first performed ballistic tests on $63.5 \mathrm{~mm}$ thick bare $\mathrm{Al}$ targets made from the same $6061 \mathrm{~T} 6$ aluminum used for the $31.6 \mathrm{~mm} \mathrm{Al}$ plates. These thicker Al plates had the same in-plane dimensions as the $31.6 \mathrm{~mm}$ plates and were impacted normally and centrally by the same spherical steel projectile as described in Section 4.1.1.3. at velocities $V_{i}$ in the range 700 to $3300 \mathrm{~m} \mathrm{~s}^{-1}$. After the tests, the targets were water jet sectioned through the center of the impact crater, and the depth of penetration (DOP) measured from the bottom of the crater to the pre-impact location of the impact surface. 
In addition, the crater diameter was measured at the original location of the impact surface.

The DOP and crater diameter are plotted in Figure 4.7 as functions of the projectile impact velocity $V_{i}$. The DOP initially increased with impact velocity reaching a local maximum of $42 \mathrm{~mm}$ at a $V_{i}$ of about $1700 \mathrm{~m} \mathrm{~s}^{-1}$. It then declined to a minimum of $30 \mathrm{~mm}$ at an impact velocity of $\sim 2100 \mathrm{~ms}^{-1}$ before starting to rise once again and reaching its previous maximum DOP value (attained at $V_{i} \approx 1700 \mathrm{~m} \mathrm{~s}^{-1}$ ) at a $V_{i}$ of about $3300 \mathrm{~m} \mathrm{~s}^{-1}$. Dehn [5] observed the same phenomena while investigating the DOP into semi-infinite plates and rationalized them as follows. The impact generates dynamic stresses within the projectile that scale with impact velocity and, at a sufficiently high impact velocity, termed the fracture threshold, the stresses surpass the strength of the projectile resulting in projectile fracture. Further increases in $V_{i}$ increase the number of the fragments and these fragments begin to spatially spread. Above a critical velocity, termed the shatter threshold [5], the increased loading area from the severely fragmented projectile causes the DOP to rapidly decrease with increasing impact velocity, and is accompanied by increases in the crater diameter. Eventually the DOP begins to rise once again, as the impact becomes a predominantly hydrodynamic event, and the $V_{i}$ range over which the DOP shows a dip is referred to as the shatter gap [5]. We observe from Figure 4.7 that our projectile/aluminum system has a shatter threshold at $\sim 1700 \mathrm{~m} \mathrm{~s}^{-1}$. Examination of the recovered projectiles revealed a fracture threshold velocity in the range of $1100 \mathrm{~m} \mathrm{~s}^{-1}$ to $1150 \mathrm{~m} \mathrm{~s}^{-1}$ after a DOP of $\sim 25 \mathrm{~mm}$. Therefore, we will be able to exploit the fragmentation of the projectile, and accompanying fragment dispersion, with our $31.6 \mathrm{~mm}$ plate when impacted at velocities above $V_{i} \sim 1150 \mathrm{~m} \mathrm{~s}^{-1}$. 


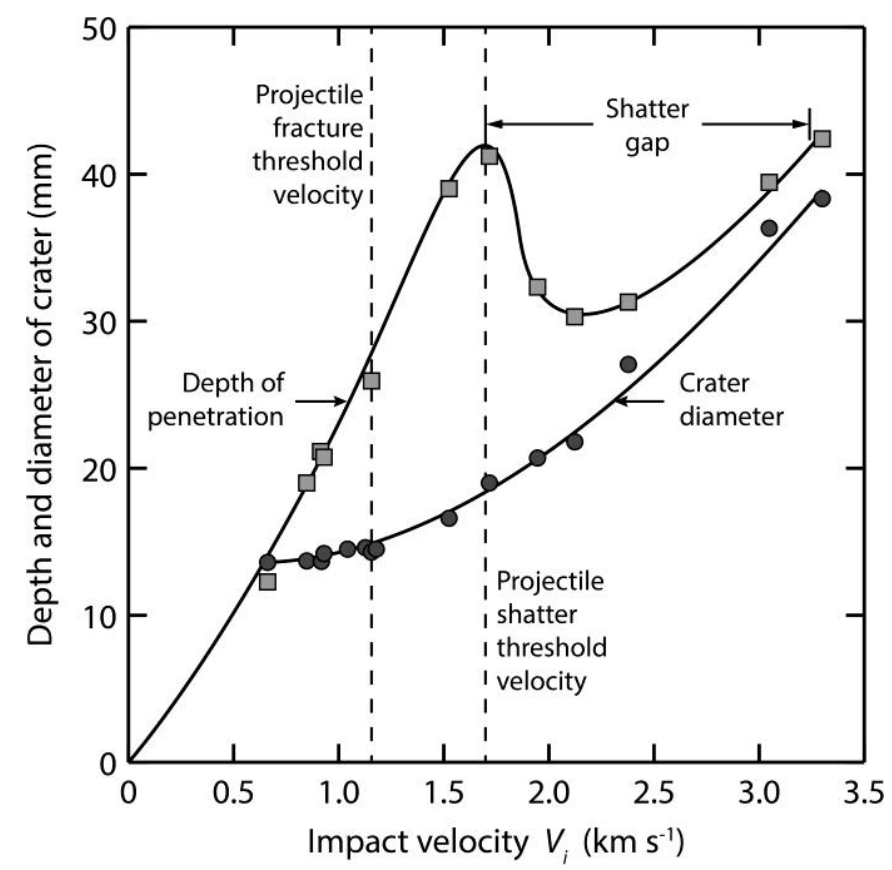

Figure 4.7. The measured depth of penetration and crater diameter as a function of impact velocity for impacts against a 63.5mm thick Al 6061-T6 plate.
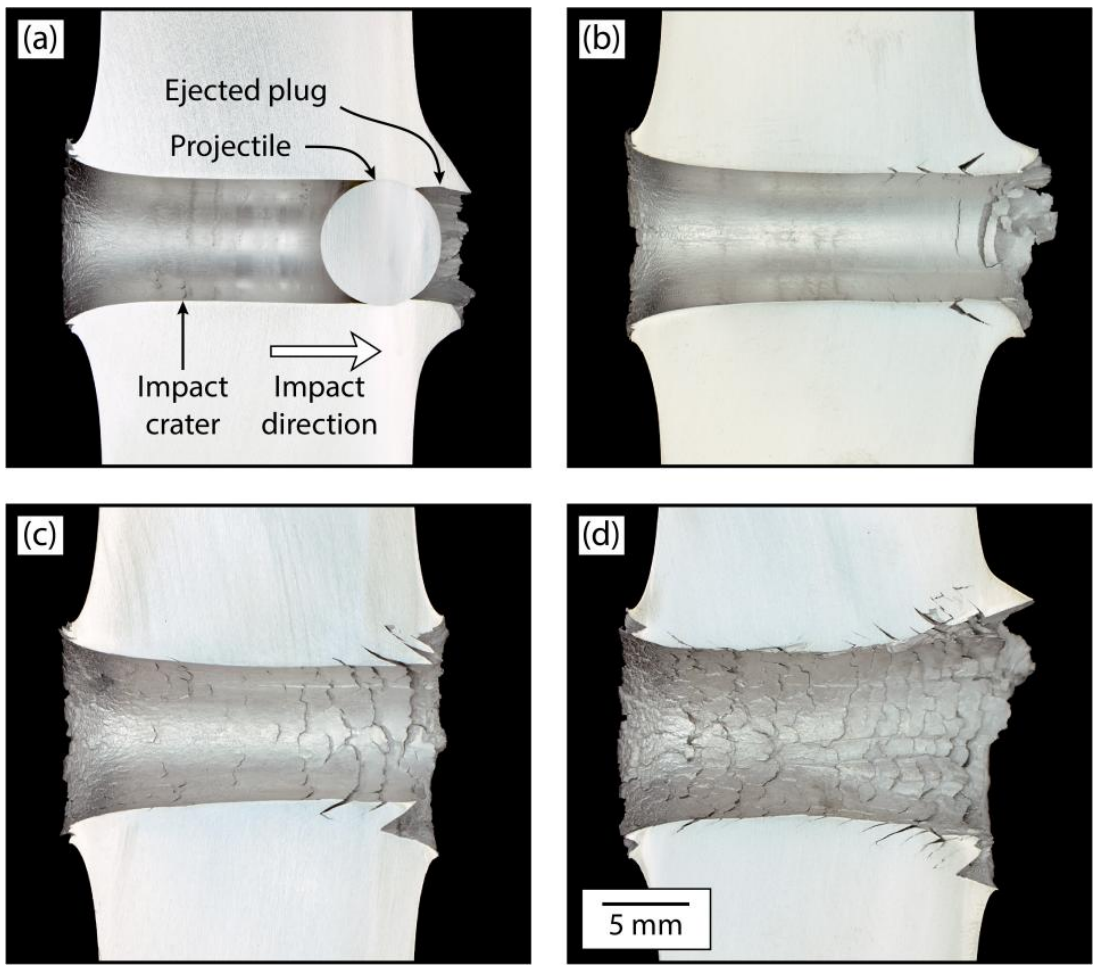

Figure 4.8. Photographs of $31.6 \mathrm{~mm}$ thick bare aluminum plates impacted at (a) $V_{i}=1090 \mathrm{~m} \mathrm{~s}^{-1}$, (b) $V_{i}=1360 \mathrm{~ms}^{-1}$, (c) $V_{i}=1530 \mathrm{~ms}^{-1}$ and (d) $V_{i}=1647 \mathrm{~ms}^{-1}$ and sectioned along a central plane. 
With the understanding of the projectile fragmentation gained from the DOP measurements on the thick Al targets, let us now revisit the ballistic measurements of the $31.6 \mathrm{~mm}$ thick Al targets in Figure 4.6. Sections through the tested targets at four selected impact velocities, $V_{i}$, are included in Figure 4.8. At $V_{i}=1090 \mathrm{~m} \mathrm{~s}^{-1} \approx V_{b l}$, Figure 4.8(a), the section shows that the spherical projectile is intact within the target and a small plug is ejected from the target with a residual velocity of about $65 \mathrm{~m} \mathrm{~s}^{-1}$. With increases in $V_{i}$, the spherical projectile exits the target and a cylindrical cavity is left behind in the target. The diameter of this cavity increases with $V_{i}$ as evident from Figure 4.8 and this is quantified in Figure 4.9, where the measured crater diameter at mid-plate thickness is plotted as a function of $V_{i}$. Note that the crater diameter was equal to the projectile diameter at low impact velocities but increased quadratically for velocities above the projectile fracture threshold of $\sim 1150 \mathrm{~m} \mathrm{~s}^{-1}$; this is attributed to the spreading of the loading caused by the fragmentation of the projectile. Moreover, the cavity develops an increasingly 'hour glass' profile and the inner surface of the cavity changes its texture; below this shatter threshold the inner surface of the cavity is smooth (see Figure 4.8(a) and (b)) but at the higher impact velocities scaling of the inner surface of the cylindrical cavity is observed. This scaling is attributed to abrasion from the fragmented projectile as it penetrated the target. A second effect of projectile shattering was the leveling-off of the projectile/ejecta residual velocity (Figure 4.6) as the shatter threshold, $V_{i} \approx 1700 \mathrm{~m} \mathrm{~s}^{-1}$, was approached. It is thus evident that projectile fragmentation is a potent mechanism to defeat an incoming projectile, and the application of Dyneema ${ }^{\circledR}$ to exploit this will be made evident in the analysis of the Dyneema ${ }^{\circledR}$ encased targets. 


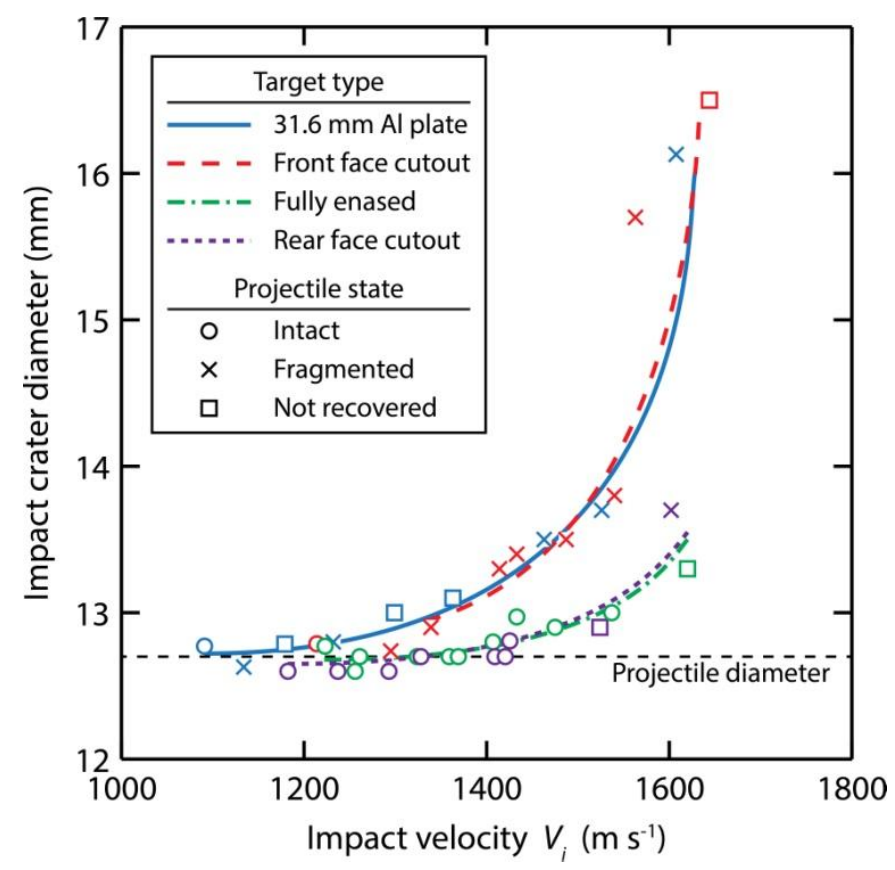

Figure 4.9. The measured crater diameter in the Al plates of the different targets as a function of impact velocity for the four model targets. The state of the projectile is indicated in each case.

A sequence of high speed images showing the dynamic deformation and penetration of the $31.6 \mathrm{~mm}$ thick Al plate impacted at $V_{i}=1300 \mathrm{~m} \mathrm{~s}^{-1}$ and $1610 \mathrm{~m} \mathrm{~s}^{-1}$ are shown in Figure 4.10(a) and (b), respectively. Figure 4.10(a) shows a profile view, and the view in Figure 4.10(b) is the so-called dead man's view (i.e. normal to the rear face). Time stamps are included in Figure 4.10 with $t=0$ corresponding to the instant that the projectile impacts the target. In both cases, a bulge is seen to form on the rear surface of the Al plate prior to the exit of the projectile/projectile fragments. The diameter of this bulge is significantly larger than the diameter of the projectile and increases from about $20 \mathrm{~mm}$ in the $V_{i}=1300 \mathrm{~m} \mathrm{~s}^{-1}$ to approximately $34 \mathrm{~mm}$ in the $1610 \mathrm{~m} \mathrm{~s}^{-1}$ case. This suggests that the bulging Al plate can serve as an effective load spreader. This effect too will be exploited in the Dyneema ${ }^{\circledR}$ encased targets to help increase the ballistic resistance of the targets. 
(a)
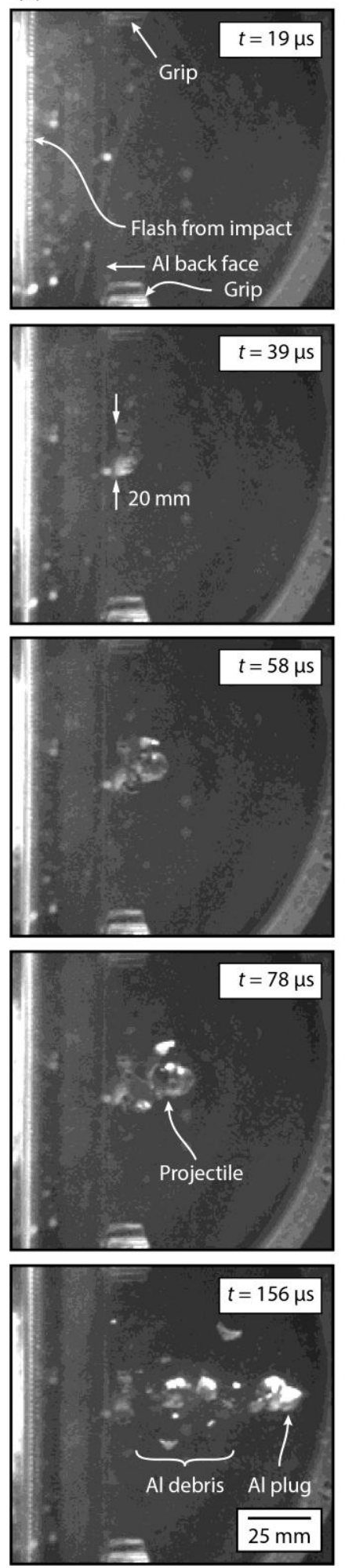

(b)
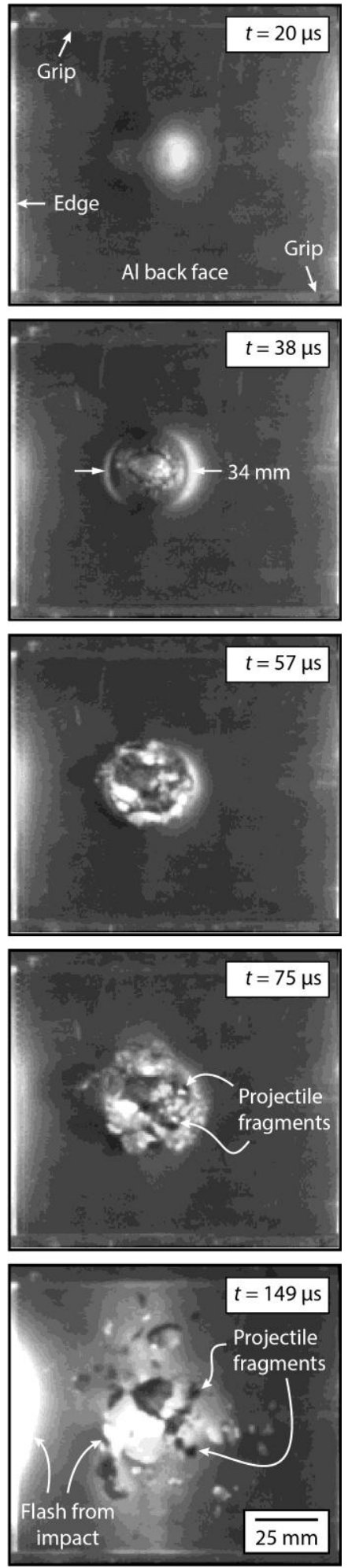

Figure 4.10. High speed image sequences showing the deformation of the bare 31.6mm thick Al target impacted at (a) $V_{i}=1300 \mathrm{~m} \mathrm{~s}^{-1}$ and (b) $V_{i}=1610 \mathrm{~m} \mathrm{~s}^{-1}$. The images in (a) are of a profile view of the rear of the target while $(b)$ shows an oblique view of the rear surface. Time $t=0$ corresponds to the instant that the projectile impacts the front face. 


\subsubsection{The front face cutout target}

In this design, the projectile directly impacts the Al plate and then penetrates through the Al plate before interacting with the rear Dyneema ${ }^{\circledR}$ face. The impact versus residual velocity plot in Figure 4.6 shows that targets with the front face cutout have the highest ballistic limit of all the targets considered here. Moreover, after a sharp rise in the residual velocity just above the ballistic limit, $V_{r}$ plateaus-out, or even decreases, with increasing $V_{i}$ over the range of impact velocities considered here. As the projectile directly impacts the bare $\mathrm{Al}$ plate and penetrates through this plate before interacting with the Dyneema ${ }^{\circledR}$ it is unsurprising that the crater diameter versus $V_{i}$ relation for this target is identical to that for the bare $\mathrm{Al}$ target, Figure 4.9. We also anticipate that similar to the bare $\mathrm{Al}$ target, the projectile incident upon this target shatters upon impact with the $\mathrm{Al}$ front face for impact velocities in excess of about $V_{i} \sim 1150 \mathrm{~m} \mathrm{~s}^{-1}$.

The superior performance of this target design is understood by considering the case of impact at $V_{i}=1430 \mathrm{~m} \mathrm{~s}^{-1}$, which is just below the ballistic limit. X-ray computed tomographic (XCT) reconstructed images of the center of the rear Dyneema ${ }^{\circledR}$ face are shown in Figure 4.11; in Figure 4.11(a) a sketch illustrating the region being imaged is shown while in Figure 4.11(b) and (c) the XCT images on two orthogonal planes are included. It is clear that (i) the projectile that has impacted the rear Dyneema ${ }^{\circledR}$ face is fragmented, (ii) this fragmented projectile is arrested after it has broken/penetrated more than half of the Dyneema ${ }^{\circledR}$ plies of the rear face laminate and (iii) the impacted area of the fragmented projectile with the rear laminate is larger than that of an intact projectile. In Section 4.3.3, similar levels of penetration of the rear Dyneema ${ }^{\circledR}$ laminate are shown to occur in the fully encased target, but at a lower impact velocity $V_{i}=1360 \mathrm{~m} \mathrm{~s}^{-1}$. 
However, in this case the projectile remained intact when it exited the Al plate and impacted the rear Dyneema ${ }^{\circledR}$ face. Thus, we argued that fragmentation of the projectile caused by the direct impact of the projectile against the high impedance $\mathrm{Al}$ face is the primary cause for the superior performance of the front face cutout targets.
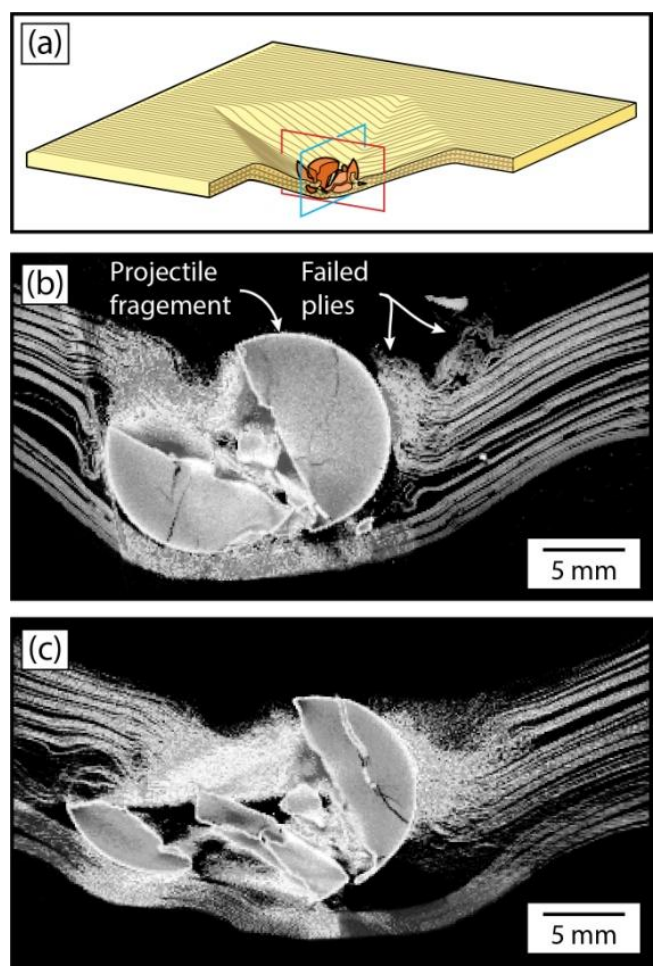

Figure 4.11. (a) A spatial reference for interpretation of the $X$-ray images shown in (b) and (c) of the rear Dyneema ${ }^{\circledR}$ laminate from a front face cutout target impacted at $V_{i}=1430 \mathrm{~m} \mathrm{~s}^{-1}$. The white areas and bright streaks are beam hardening artifacts ${ }^{4}$.

High speed images of the deformation of the front face cutout target impacted at $V_{i}=1430 \mathrm{~m} \mathrm{~s}^{-1}$ are shown in Figure 4.12 with the images in Figure 4.12(a) showing the deformation of the rear face via a side profile, while Figure 4.12(b) and (c) show oblique views of the rear and front faces, respectively. The images are time synchronized, and time marks are indicated on the images, with $t^{\prime}=0$ corresponding to the instant that the

\footnotetext{
${ }^{4}$ High atomic weight materials preferentially absorb low energy $\mathrm{x}$-rays causing a shift of the polychromatic beam to a higher mean $\mathrm{x}$-ray energy. Therefore, $\mathrm{x}$-ray absorption per unit length is most potent for shorter beam paths through a given material. The result is bright streaks at the edge of an object.
} 
deflection of the rear Dyneema ${ }^{\circledR}$ face commenced. Recall that prior to the projectile breaking through the Al plate, the Al plate bulges and petals, Figure 4.10. This bulging of the Al plate initiates the motion of the rear Dyneema ${ }^{\circledR}$ face, and it is thus surmised that $t^{\prime}=0$ corresponds to the initiation of the bulging of the rear of the Al plate rather than the impact of the projectile/projectile fragments on the rear Dyneema ${ }^{\circledR}$ face. The side profile of the rear Dyneema ${ }^{\circledR}$ face shows a triangular profile with the base of the triangle and the height of the triangle increasing with time. The two hinges at the base of the triangle each moved outwards towards the edge of the plate at a speed of approximately $300 \mathrm{~m} \mathrm{~s}^{-1}$. From the oblique rear view, Figure 4.12(b), the deflected profile is seen to be pyramidal in shape, with the ridges of the pyramid deflection (lines of pyramidal face pair contact) aligned with the $0^{\circ} / 90^{\circ}$ fiber orientations. Thus, the deformation of the Dyneema ${ }^{\circledR}$ laminate is highly anisotropic with the hinges travelling significantly faster along the fiber directions (which are aligned with the black marker lines) compared to their velocity at $45^{\circ}$ to these lines. These observations are all consistent with those reported in [2] for a Dyneema ${ }^{\circledR}$ laminate directly impacted by a spherical projectile. 
(a)

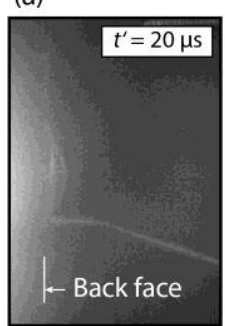

(b)

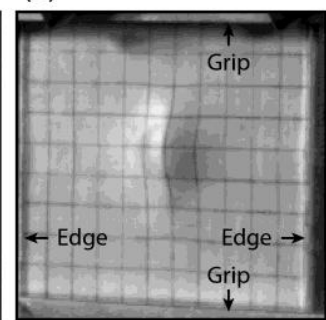

$t^{\prime}=60 \mu \mathrm{s}$
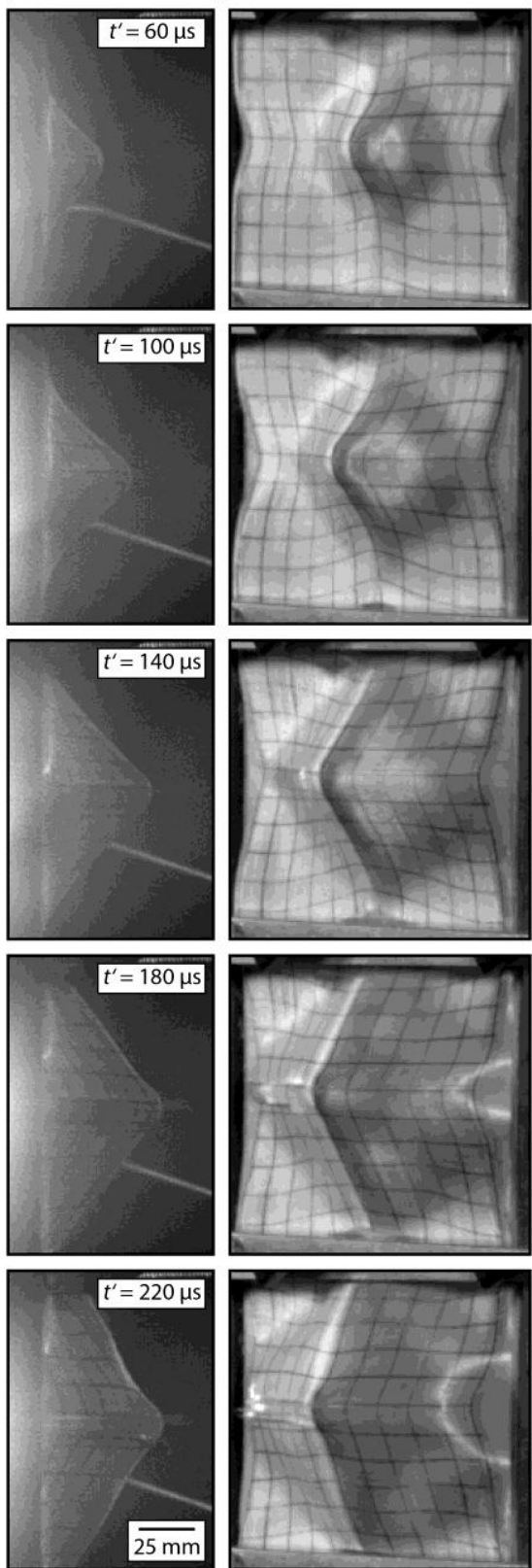

(c)
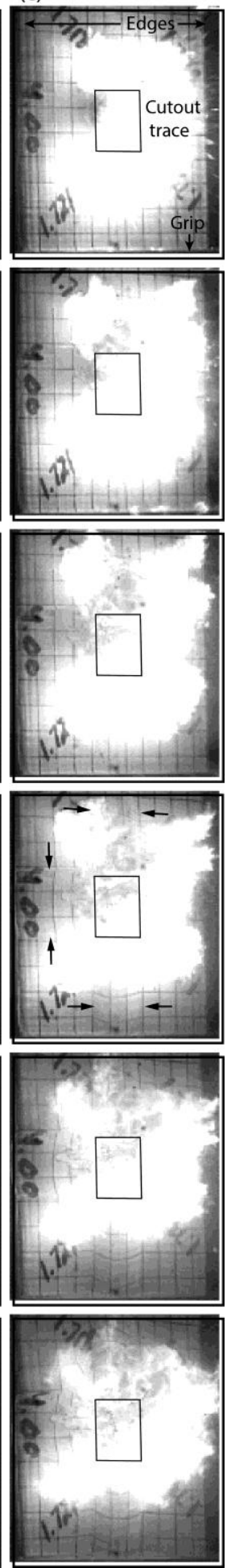

Figure 4.12. Synchronized high speed image sequences of the front face cutout target impacted at $V_{i}=1430 \mathrm{~m} \mathrm{~s}^{-1}$. Three different views are shown: (a) a profile view, and oblique views of the $(b)$ rear and (c) front surfaces. A $12.7 \mathrm{~mm}$ spaced grid pattern is drawn on the Dyneema ${ }^{\circledR}$. The rectangular box in shown in (c) to indicate the position of the cutout section from the front face. Time $t^{\prime}=0$ corresponds to the instant that the deformation of the rear Dyneema ${ }^{\circledR}$ face initiates. 
Note that very large transverse deflections of the Dyneema ${ }^{\circledR}$ rear face are seen in Figure 4.12. In Section 4.3.3.1. we shall shown that these large out-of-plane deflections of the Dyneema ${ }^{\circledR}$ laminate are not primarily due to the straining of the Dyneema ${ }^{\circledR}$ laminate, but rather to pull-in of the Dyneema ${ }^{\circledR}$ from the sides and front of the target. This pull is evident in oblique front view images, Figure $4.12(\mathrm{c})^{5}$, where the grid lines curve near the cutout section of the Dyneema ${ }^{\circledR}$ (within the region indicated by the arrows) as the Dyneema ${ }^{\circledR}$ is pulled to accommodate the transverse deflection of the rear face.

\subsubsection{The baseline fully encased target}

The measured residual velocity of the projectile/ejecta versus projectile impact velocity plotted in Figure 4.6 shows that fully encasing the Al plate in Dyneema ${ }^{\circledR}$ results in a ballistic performance superior to the bare $\mathrm{Al}$ plate but inferior to the front face cutout target. Moreover, unlike the bare $\mathrm{Al}$ plate and the front face cutout targets, the residual velocity does not plateau out, but continues to increase approximately linearly with $V_{i}$ up to the highest $\left(1600 \mathrm{~m} \mathrm{~s}^{-1}\right)$ impact velocity investigated here. Further, the crater diameter plotted as a function of $V_{i}$ in Figure 4.9 clearly shows that for a given impact velocity, a cylindrical cavity with a smaller diameter is formed in the fully encased targets compared to the bare Al plates or the front face cutout targets; a difference that increases with increasing $V_{i}$.

\footnotetext{
${ }^{5}$ The bright flash in the front face high speed photographs is due to impact luminescence when the projectile impacts the bare $\mathrm{Al}$ plate.
} 

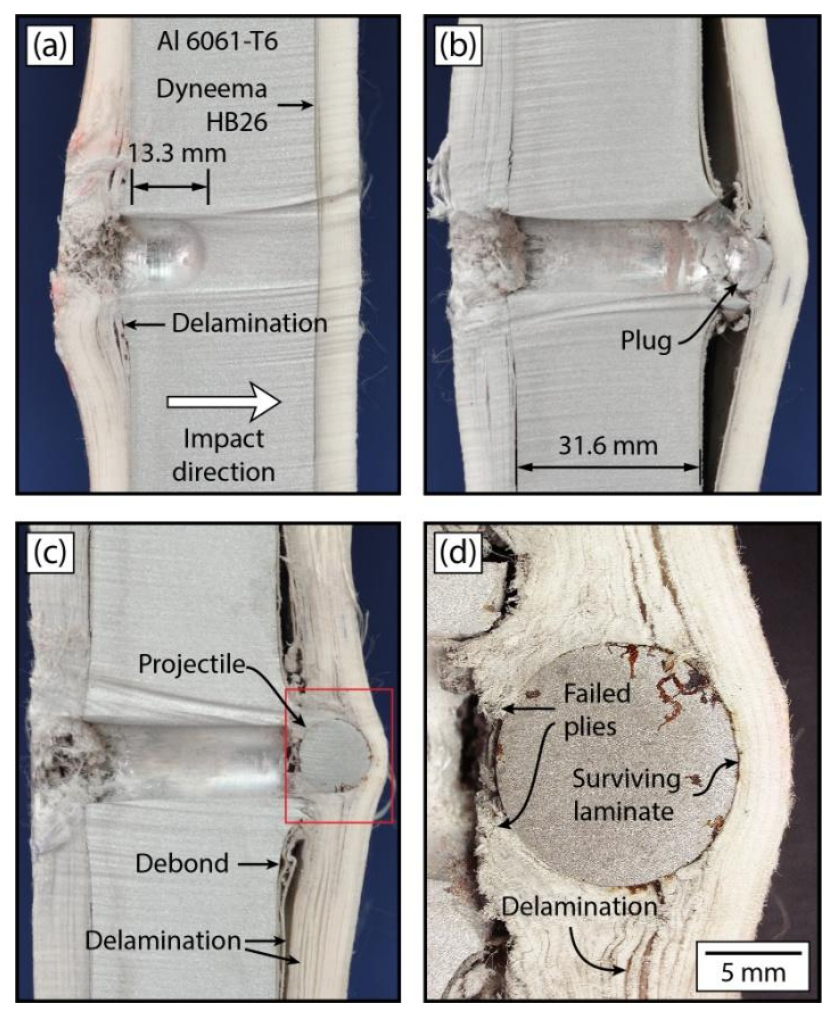

Figure 4.13. Photographs of the baseline fully encased target impacted at (a) $V_{i}=800 \mathrm{~m} \mathrm{~s}^{-1}$, (b) $V_{i}=1220 \mathrm{~m} \mathrm{~s}^{-1}$ and $(c) V_{i}=1360 \mathrm{~m} \mathrm{~s}^{-1}$ and sectioned along a central plane. A magnified view of the region around the arrested projectile from $(c)$ is shown in $(d)$. The images have been annotated to highlight salient features.

These observations are rationalized by noting that the Dyneema ${ }^{\circledR}$ on the front face acts as a low impedance "soft" surface to the incoming projectile. This results in the shatter threshold increasing significantly beyond the $V_{i} \approx 1150 \mathrm{~m} \mathrm{~s}^{-1}$ value of the bare $\mathrm{Al}$ plate (as in the case of the bare Al plate or the front face cutout targets). This increase in the shatter threshold is confirmed in Figure 4.13 where photographs of sections through the mid-plane of the fully encased targets are shown for three $V_{i}$ values below the ballistic limit. It is clear that even for the case of $V_{i}=1360 \mathrm{~m} \mathrm{~s}^{-1}$ the projectile remains intact in the fully encased target while the projectile had fragmented at a lower impact velocity for the front face cutout target (recall Figure 4.11). The retention of a spherical projectile has three consequences: 
(i) A cylindrical cavity of smaller diameter is created in the Al plate as the loading remains concentrated.

(ii) The loading on the rear Dyneema ${ }^{\circledR}$ laminate is mainly via the intact spherical projectile compared to the distributed loading by the fragmented projectile in the front face cutout target. This reduces the ability of the Dyneema ${ }^{\circledR}$ rear face to arrest the projectile.

(iii) Since the projectile remains intact, the residual velocity does not plateau out for the values of $V_{i}$ reached here. We anticipate that at higher impact velocities, where the shatter threshold for a Dyneema ${ }^{\circledR}$ faced $\mathrm{Al}$ plate is attained, a plateauing of the residual velocity would be observed.

By comparing the ballistic performance of the front face cutout and fully encased targets it is clear that while the Dyneema ${ }^{\circledR}$ on the front face aids in reducing the velocity of the incoming projectile ${ }^{6}$, this front face Dyneema ${ }^{\circledR}$ effectively reduces the ability of the rear face Dyneema ${ }^{\circledR}$ to catch the projectile by keeping the projectile intact. Overall, this detrimental effect of the front face Dyneema ${ }^{\circledR}$ is larger and hence the fully encased targets have a lower ballistic performance compared to the front face cutout targets.

\footnotetext{
${ }^{6}$ The role of the Dyneema ${ }^{\circledR}$ on the front face in reducing the velocity of the incoming projectile will be made explicit in Sections 4.3.4 and 4.4.1 where the rear face cutout target is discussed.
} 

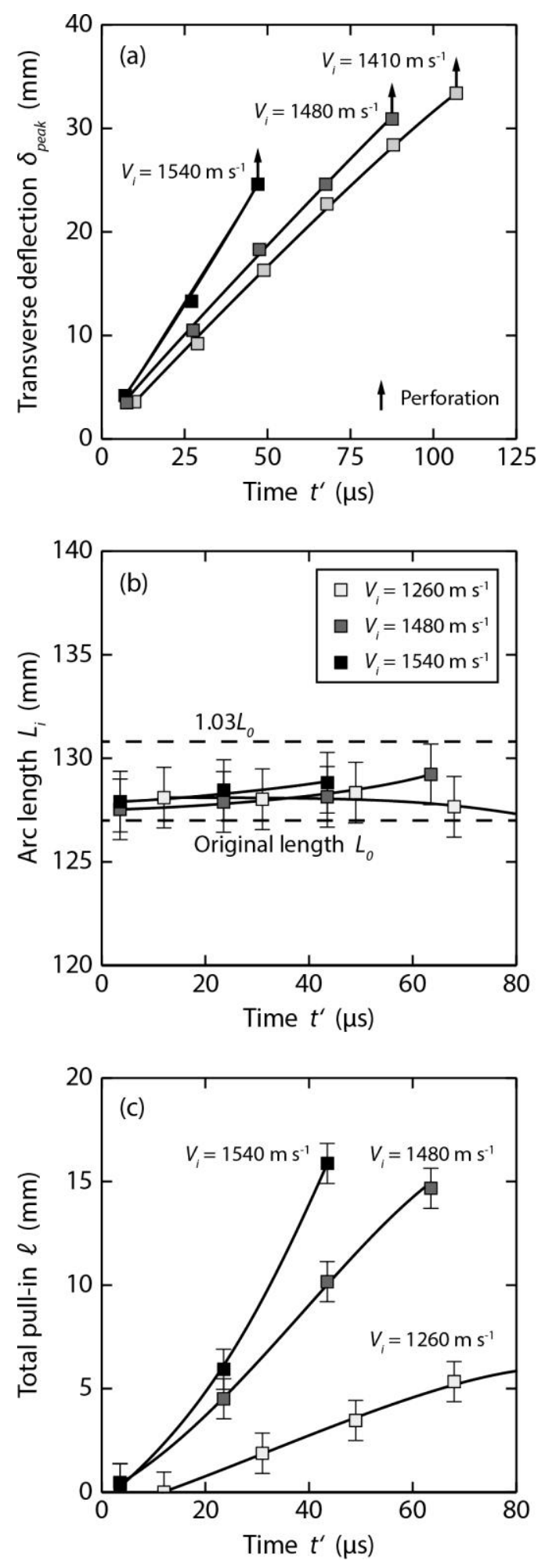

Figure 4.14. (a) The measured transverse deflection $\delta$ of the mid-span of the Dyneema ${ }^{\circledR}$ rear face, (b) the pull-in length, $\ell$, and (c) deformed rear laminate length $L$ of the baseline fully encased target as function of time $t^{\prime}$ for three select values of the impact velocity. The original length $L_{0}$ of the undeformed laminate is indicated in (b), and time $t^{\prime}=0$ corresponds to the instant that the deformation of the rear Dyneema ${ }^{\circledR}$ face initiates. 


\subsubsection{Extension of the Dyneema ${ }^{\circledR}$ plies}

The transverse deflection $\delta_{\text {peak }}$ of the mid-span of the Dyneema ${ }^{\circledR}$ rear face is plotted in Figure 4.14(a) as a function of time $t^{\prime}$ for three select values of the impact velocity $V_{i}$. The very large transverse deflections (maximum deflections $\delta_{\text {max }} \approx 30 \mathrm{~mm}$ over a span of $L_{0} \approx 130 \mathrm{~mm}$ ) suggest that the extensional strains in the Dyneema ${ }^{\circledR}$ plies are $\approx 2\left(\delta_{\max } / L_{0}\right)^{2}=46 \%$ which is far in excess of the dynamic Dyneema ${ }^{\circledR}$ fiber failure strains reported in [6]. The reason for this discrepancy is that in making the above extensional strain estimate we assume that the rear Dyneema ${ }^{\circledR}$ face is rigidly held at the edges of the plate and does not pull in. However, the oblique high-speed images in Figure 4.15(b) (note the deformed grid pattern near the sample edges) show that the grid pattern on the rear face near the edges translates and that the Dyneema ${ }^{\circledR}$ is pulled inwards from the edges by the mechanism sketched in Figure 4.15(a). This pull-in needs to be corrected for in order to make an accurate estimate of the actual extensional strain within the plies of the rear Dyneema ${ }^{\circledR}$ face. 

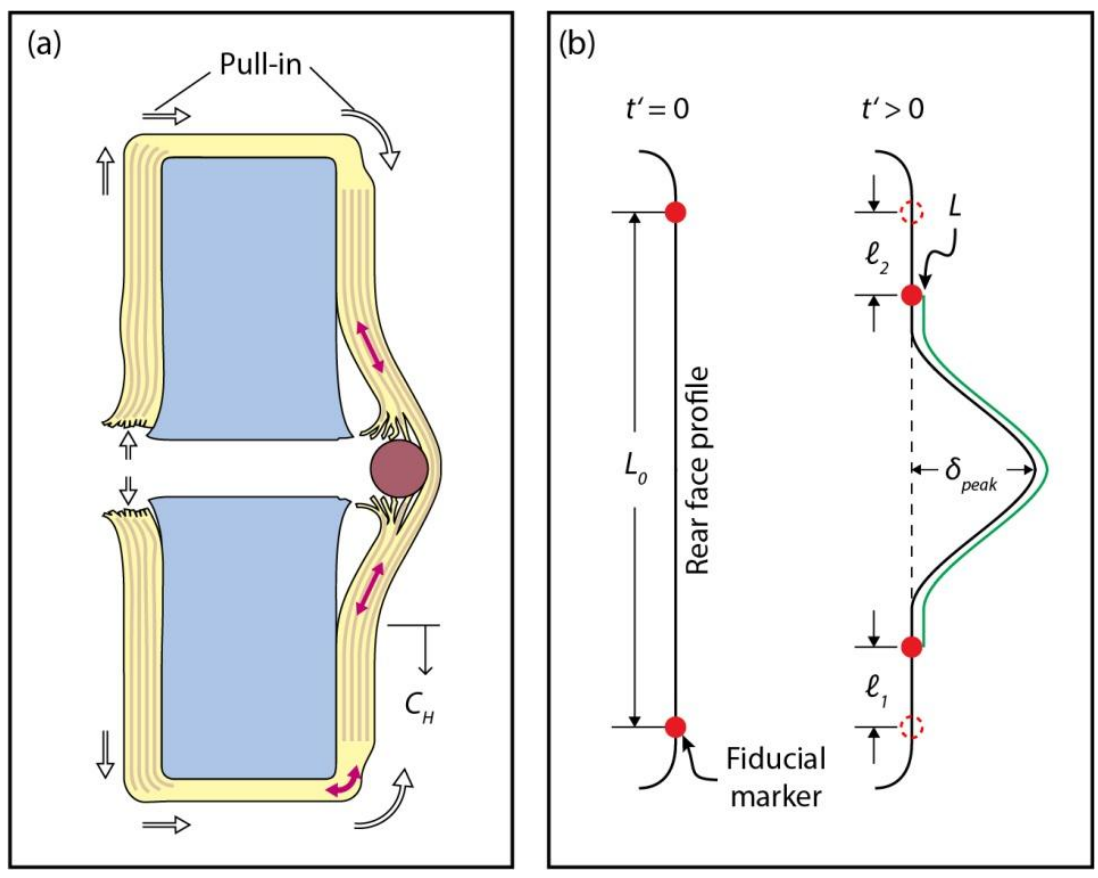

Figure 4.15. Schematic showing (a) the pull-in of the Dyneema ${ }^{\circledR}$ encasing around the Al plate which results in large deflections of the rear Dyneema ${ }^{\circledR}$ face and $(b)$ the definition of the pull-in length $\ell$ and deformed rear laminate length $L$ as measured from the fiducial markers on the rear Dyneema ${ }^{\circledR}$ face.

Consider the cross-sectional sketch of the rear Dyneema ${ }^{\circledR}$ face in Figure 4.15(b), where we have indicated the position of a fiducial marker near the edge of the plate in both the undeformed and transient deformed configurations. The grid lines on the rear face serve as these fiducial markers, and we choose grid lines that are initially spaced a distance $L_{0}$ apart. As the Dyneema ${ }^{\circledR}$ is pulled-in from the front face towards the rear, these fiducial markers move inwards by $\ell_{1}$ and $\ell_{2}$, respectively such that the total pull-in distance $\ell=\ell_{1}+\ell_{2}$. The section of the Dyneema ${ }^{\circledR}$ rear face of initial length $L_{0}$ has changed to a length $L_{i}$ in the deformed configuration, where $L_{i}$ is the arc-length between the fiducial markers in the deformed configuration. The technique used to measure $\ell$ and $L_{i}$ from the oblique and profile high speed image views is detailed in the Appendix B. 
The measured values of $L_{i}$ and $\ell$ as a function of time $t^{\prime}$ are plotted in Figure 4.14(b) and (c), respectively, for three values of the impact velocity $V_{i}$. It is clear that within experimental error $L_{i} \leq 1.03 L_{0}$ throughout the deformation history. This clearly shows that the extension of the Dyneema ${ }^{\circledR}$ plies is no more than the measured dynamic failure strain of the Dyneema ${ }^{\circledR}$ yarns by Russell et al. [6]. However, $\ell$ increases monotonically with $t^{\prime}$ (Figure 4.14(c)), and thus it is concluded that the large deflections of the rear face are largely accommodated by pull-in at the edges rather than just elastic extensional straining of the Dyneema ${ }^{\circledR}$ plies.

\subsubsection{The rear face cutout target}

The rear face cutout targets had a significantly lower ballistic performance compared to the fully encased or front face cutout targets (Figure 4.6). In fact, the ballistic limit was only $100 \mathrm{~m} \mathrm{~s}^{-1}$ higher than the bare Al plates. This can be understood in terms of the insights gained from the discussions in Sections 4.3.1 to 4.3.3. Recall that the key to the superior performance of the front face cutout targets stemmed from the projectile fragmenting upon impacting the bare Al plate at high velocities. These spatially and temporally dispersed fragments then penetrate through the Al plate and subsequently impact the rear laminate. When Dyneema ${ }^{\circledR}$ was added on the front face, as seen with the fully encased target, the projectile did not fragment, and thus the aluminum plate and rear laminate of this target were loaded by the intact projectile, which applied a highly localized pressure to the Dyneema ${ }^{\circledR}$ laminate. This resulted in a reduced ballistic performance compared to the front face cut out target. 
The rear face cutout target does not invoke any of the mechanisms that enhance the ballistic performance of the targets, viz. it has Dyneema ${ }^{\circledR}$ on the front face that prevents the fragmentation of the projectile and it does not have a Dyneema ${ }^{\circledR}$ rear face to catch the projectile that exits the Al plate. This is clearly seen in the high speed images of Figure 4.16 which show an oblique view of the rear face of the rear face cutout target impacted at $V_{i}=1420 \mathrm{~m} \mathrm{~s}^{-1}$. An intact projectile exits the target after penetrating through the Al plate.
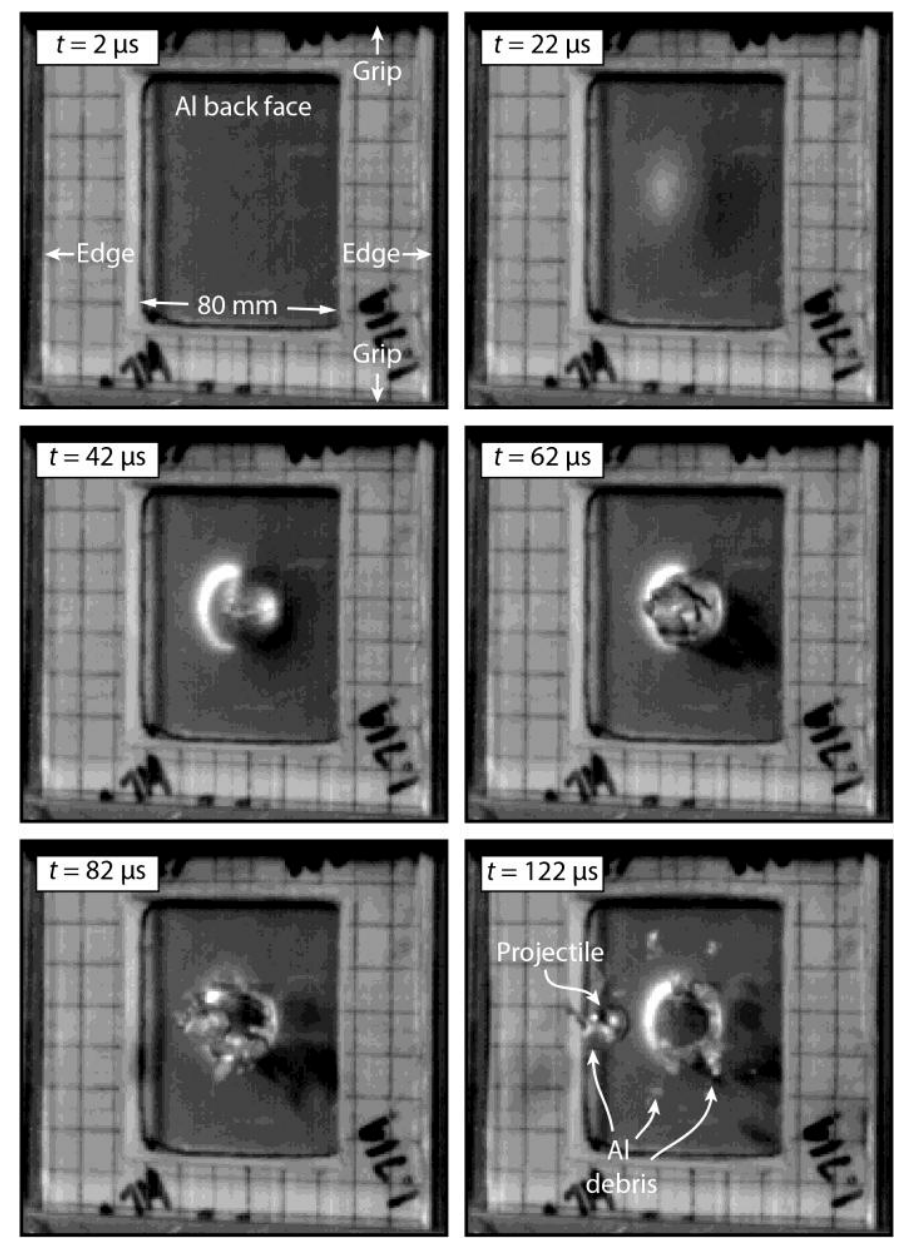

Figure 4.16. High speed images showing an oblique view of the deformation of the rear surface of the rear face cut out specimen impacted at $V_{i}=1420 \mathrm{~m} \mathrm{~s}^{-1}$. A $12.7 \mathrm{~mm}$ spaced grid pattern is drawn on the Dyneema ${ }^{\circledR}$. Time $t=0$ corresponds to the instant that the projectile impacts the target. 


\subsection{Discussion of Dyneema penetration mechanisms}

The three different types of Dyneema ${ }^{\circledR}$ encased targets investigated above have revealed that Dyneema ${ }^{\circledR}$ on the front and the rear face have rather contrasting effects on the ballistic resistance of the targets. In order to better understand these effects we proceed to quantify the ballistic performance of the Dyneema ${ }^{\circledR}$ faces in these samples using a methodology introduced by Karthikeyan et al. [2] which quantifies the progressive failure of the Dyneema ${ }^{\circledR}$.

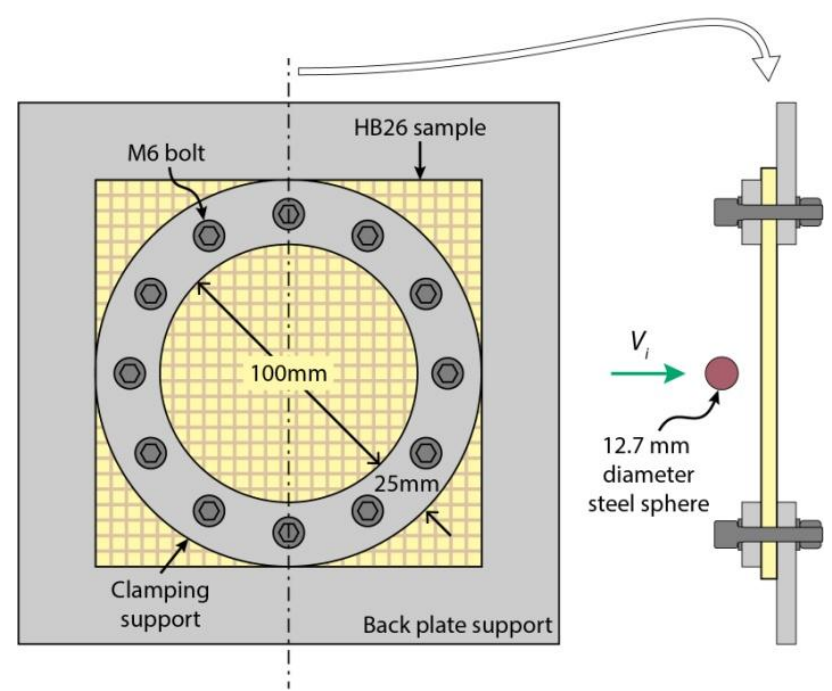

Figure 4.17. Schematic of the setup used by Karthikeyan et al. [2] to measure the ballistic performance of HB26 Dyneema ${ }^{\circledR}$ plates impacted by a $12.7 \mathrm{~mm}$ diameter steel sphere.

Let us begin by first summarizing the results of Karthikeyan et al. [2] who impacted $150 \mathrm{~mm}$ square, $\sim 6 \mathrm{~mm}$ thick, edge clamped HB26 Dyneema ${ }^{\circledR}$ plates by a 12.7 mm diameter spherical steel ball as sketched in Figure 4.17. Karthikeyan et al. [2] observed that penetration of the Dyneema ${ }^{\circledR}$ plate by this spherical projectile occurred in a progressive manner such that an increasing number of plies fractured directly under the impact site with increasing impact velocity until all plies fractured at the ballistic limit to allow the projectile to perforate the plate. The measurements of Karthikeyan et al. [2] are 
replotted in Figure 4.18(b) in terms of the fraction of perforated plies in the laminate versus the projectile impact velocity $V_{i}$. Ply fracture in the edge clamped plate initiated at an impact velocity $V_{i} \approx 250 \mathrm{~m} \mathrm{~s}^{-1}$, and the fraction of plies perforated then increased with $V_{i}$ until all plies had fractured under the projectile at the ballistic limit of about $445 \mathrm{~m} \mathrm{~s}^{-1}$. We emphasize that in the experiments of Karthikeyan et al. [2] no measurable deformation of the projectile occurred over the entire velocity range investigated.

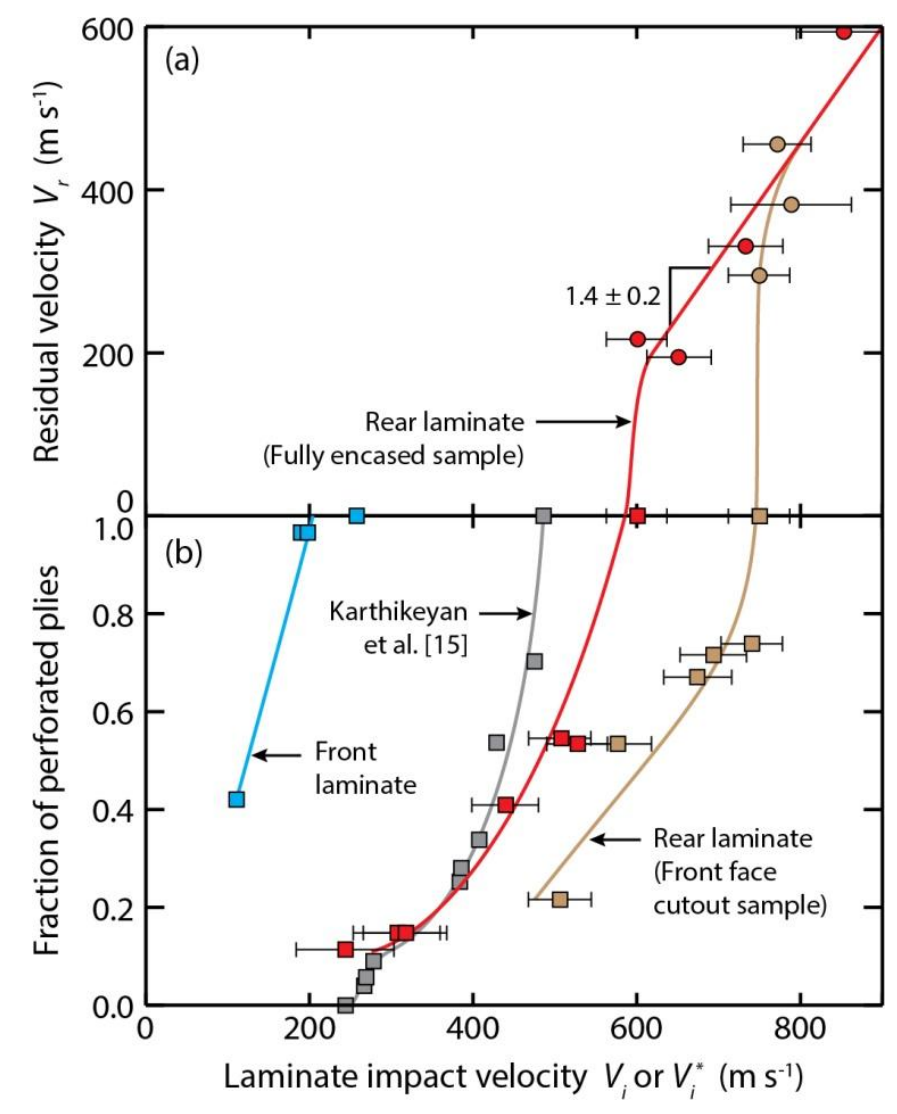

Figure 4.18. The measured (a) projectilelejecta residual velocity (circles-o-) and (b) fraction of perforated plies (squares - $\square-$ ) as a function of the velocity impacting the laminate on the front and rear sides of the targets. In $(b)$ we include data of a similar thickness edge clamped HB26 plate data from [2].

The HB26 plates investigated by Karthikeyan et al. [2] are comparable in terms of size and areal mass to the Dyneema ${ }^{\circledR}$ front and rear face laminates of the targets studied here. Moreover, the projectile used by Karthikeyan et al. [2] was identical to the 
projectile used in this study, and we can therefore directly compare our measurements with those of Karthikeyan et al. [2]. In order to make these comparisons we note that the Dyneema $^{\circledR}$ faces in the targets used here fall into two categories:

(i) The Dyneema ${ }^{\circledR}$ on the front face (in the fully encased and rear face cutout targets) is back supported by the Al plate and cannot undergo significant transverse deflection as illustrated in Figure 4.19(a).

(ii) The Dyneema ${ }^{\circledR}$ on the rear face (in the fully encased and front face cutout targets) can deflect under the projectile and pull in from the edges as illustrated in Figure 4.19(b); this case is expected to be very similar to the situation in the experiments of Karthikeyan et al. [2].

Thus each of these will be considered in turn.

\subsubsection{Dyneema plates resting on a strong foundation}

Impact experiments on the fully encased and rear face cutout targets at impact velocities much below the ballistic limit were used to measure the penetration response of the Dyneema ${ }^{\circledR}$ on the front faces of these targets and thereby infer the response of Dyneema ${ }^{\circledR}$ plates resting on a strong foundation. The measured fraction of plies penetrated in these tests as a function of $V_{i}$ is included in Figure 4.18(b). It is clear that the projectile penetrates the supported Dyneema ${ }^{\circledR}$ plate with relative ease. The plies of the plate begin to perforate by $V_{i} \approx 110 \mathrm{~m} \mathrm{~s}^{-1}$ with complete perforation of the plate (i.e. ballistic limit) being attained at $V_{i} \approx 200 \mathrm{~m} \mathrm{~s}^{-1}$. These values are at least a factor of two less than those measured by Karthikeyan et al. [2] for an edge clamped plate as seen clearly in Figure 4.18(b). 
(a)
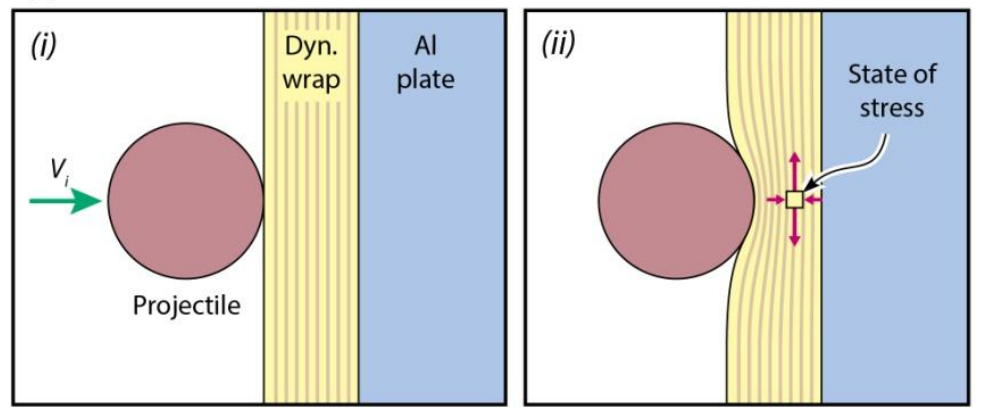

(b)

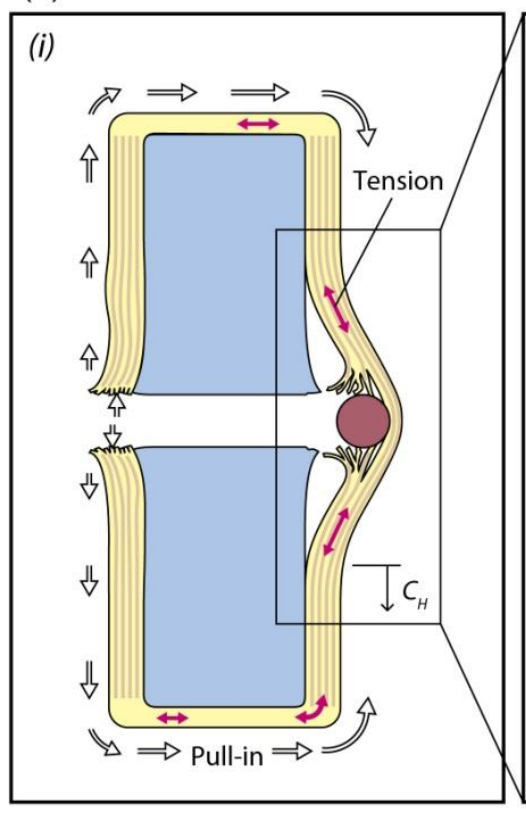

(ii)
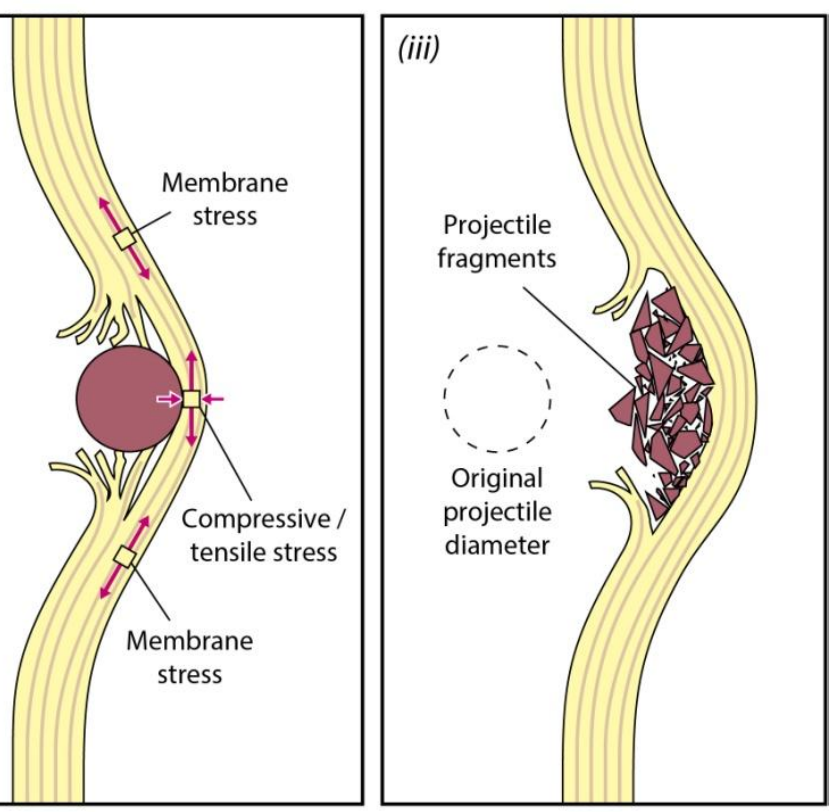

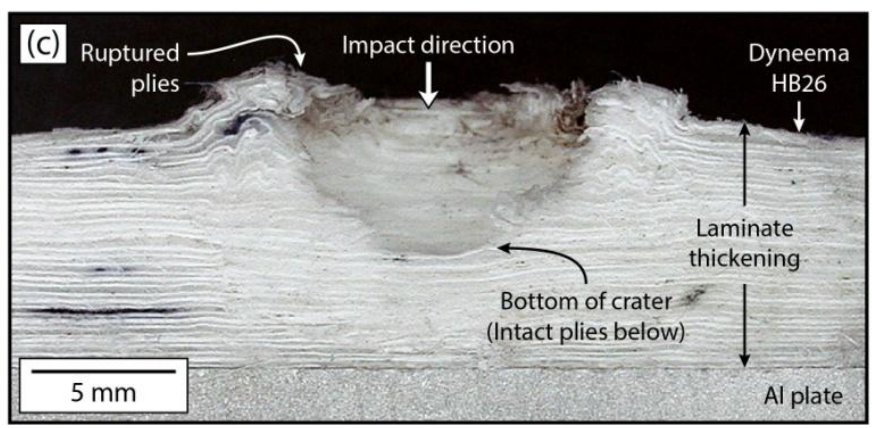

Figure 4.19. (a) Schematic showing the sequence of events for the perforation of the Dyneema ${ }^{\circledR}$ on the front face of the model targets. (b) Schematic illustrating the pull-in of the Dyneema ${ }^{\circledR}$ as the projectile impacts the rear Dyneema ${ }^{\circledR}$ face of the model targets. Magnified views of this impact event by either an intact or fragmented projectile are included. (c) A photograph of the central section through the fully encased specimen impacted at $V_{i}=110 \mathrm{~m} \mathrm{~s}^{-1}$ showing the partial perforation of the Dyneema ${ }^{\circledR}$ front laminate.

A photograph of a central section through the fully encased specimen impacted at $V_{i}=110 \mathrm{~m} \mathrm{~s}^{-1}$ showing the partially perforated front Dyneema ${ }^{\circledR}$ face is included in Figure 
4.19(c). It is clear that the perforation occurred with negligible deformation of the supporting Al plate. We thus conclude that the fiber stretching mechanism proposed by Phoenix and Porwal [7] could not have been activated during this impact event as the Dyneema $^{\circledR}$ plate was unable to deflect in a string-like mode as sketched in Figure 1.4. Rather, fracture of the Dyneema ${ }^{\circledR}$ plies seems to have occurred under compressive loading by the projectile via the indirect tension mechanism analyzed by Attwood et al. [8] (and initially hypothesized by Woodward et al. [9]). In this mechanism the applied transverse compressive stress results in shear stress forming between the alternating $0^{\circ}$ and $90^{\circ}$ plies due to the anisotropic deformation nature of these plies. These shear stresses generate fiber tension via a shear lag mechanism, and it is this fiber tension that we hypothesize causes the perforation of the plies as indicated in Figure 4.19(a).

\subsubsection{Edge supported Dyneema ${ }^{\circledR}$ plates}

The rear faces of the fully encased and front face cutout targets are impacted by the projectile/ejecta that comes through the $\mathrm{Al}$ plate. Thus, in order to make a fair comparison we define $V_{i}^{*}$ to be the velocity of the projectile/ejecta that impacts this rear Dyneema $^{\circledR}$ face. For a given projectile velocity $V_{i}$ the corresponding velocity $V_{i}{ }^{*}$ is estimated as follows:

(i) Fully encased targets: The projectile in these targets first penetrates the Dyneema $^{\circledR}$ on the front face and then the $\mathrm{Al}$ plate before it impacts the Dyneema $^{\circledR}$ rear face. Thus for a given $V_{i}, V_{i}^{*}$ is given by the residual velocity of the projectile as it exits the rear face cut-out targets. We thus calculated $V_{i}^{*}$ by interpolating the residual velocity data for the rear face cutout targets in Figure 4.6. 
(ii) Front face cutout targets: The projectile impacting these targets only penetrates the $\mathrm{Al}$ plate before it impacts the Dyneema ${ }^{\circledR}$ rear face. Thus for a given $V_{i}, V_{i}^{*}$ is given by the residual velocity of the projectile as it exits the bare $\mathrm{Al}$ targets. We thus calculated $V_{i}^{*}$ by interpolating the residual velocity data for the bare Al targets in Figure 4.6.

The measurements of the fraction of the perforated plies of the rear Dyneema ${ }^{\circledR}$ faces as a function of $V_{i}^{*}$ are included in Figure 4.18(b) for both the fully encased and front face cutout targets. Intriguingly the two sample types do not overlap and also differ from those of Karthikeyan et al. [2]. We thus discuss them in turn to clarify the mechanisms responsible for these discrepancies.

Fully encased target: The ballistic response is very similar to that observed by Karthikeyan et al. [2] until approximately $40 \%$ of the plies are penetrated. Subsequently, the rear Dyneema ${ }^{\circledR}$ face of the fully encased target seems to have an enhanced ballistic performance compared to the plates of Karthikeyan et al. [2], i.e. the rear Dyneema ${ }^{\circledR}$ face of the fully encased target needs to be impacted at a higher velocity to achieve the same level of perforation as in [2]. To rationalize this, consider the sketch in Figure 4.20(a) where the penetration of the projectile through the fully encased target is illustrated. Following the high speed images in Figure 4.16, we expect that the Al plate bulges at the rear prior to the projectile breaking through. This large radius of curvature bulging of the Al plate results in the rear Dyneema ${ }^{\circledR}$ face acquiring a velocity and deflecting prior to impact by the projectile that emerges through the bulge. This is indicated in Figure 4.20(b), where we sketch the mid-span deflection $\delta_{\text {peak }}$ of the rear Dyneema ${ }^{\circledR}$ face as a function of time $t^{\prime}$. The point at which the projectile impacts the Dyneema ${ }^{\circledR}$ face is also 
indicated (red circles) and the slope of the $\delta_{\text {peak }}$ versus $t^{\prime}$ curve at this instant is the velocity of the Dyneema ${ }^{\circledR}$ face at the instant of impact; we refer to this velocity as $\dot{\delta}_{D}$. With increasing $V_{i}$ (and consequently $V_{i}^{*}$ ), $\dot{\delta}_{D}$ increases and the relative velocity between the projectile and plate at the instant of impact, $V_{i}^{*}-\dot{\delta}_{D}$ increases at a slower rate in these fully encased specimens compared to that in the Karthikeyan et al. [2] experiments where the Dyneema ${ }^{\circledR}$ plate was always stationary prior to impact. The key driver to perforation of the Dyneema ${ }^{\circledR}$ plate is the relative velocity between the projectile and the Dyneema ${ }^{\circledR}$ plate. Recall that we have plotted the perforation of the Dyneema ${ }^{\circledR}$ in terms of the impact velocity $V_{i}$ or $V_{i}^{*}$ rather than relative velocity between the Dyneema ${ }^{\circledR}$ and the projectile. It is this choice of loading parameter ${ }^{7}$ that gives the impression of the higher ballistic performance of the rear Dyneema ${ }^{\circledR}$ face in the fully encased target compared to experiments of Karthikeyan et al. [2]. The opportunity to pre-accelerate the Dyneema ${ }^{\circledR}$ plate into motion prior to the impact of the projectile enhances the ballistic performance of the Dyneema ${ }^{\circledR}$ and is a feature that might be exploitable in the design of composite protection systems.

\footnotetext{
${ }^{7}$ We are unable to directly measure the relative velocity $V_{i}^{*}-\dot{\delta}_{D}$ and thus have shown all the results in terms of the impact velocity $V_{i}$ or $V_{i}^{*}$.
} 
(a)

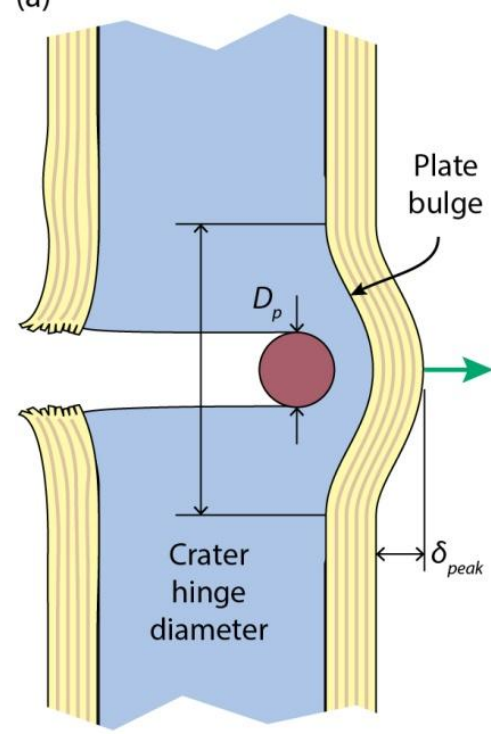

(b)

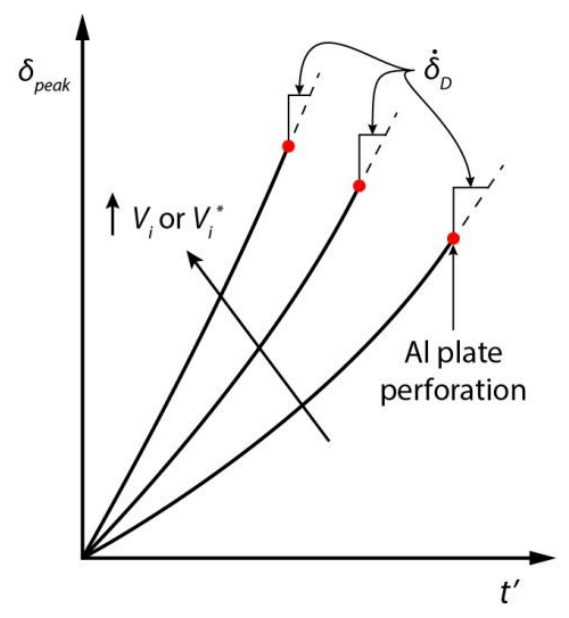

Figure 4.20. (a) A schematic illustrating the penetration of the projectile through the fully encased target showing the bulging of the rear surface of the Al plate which accelerates the Dyneema ${ }^{\circledR}$ rear face prior to impact by the projectile. (b) A schematic illustrating the deflection, $\delta_{\text {peak }}$, of the rear Dyneema ${ }^{\circledR}$ face as a function of time $t^{\prime}$. The point at which the projectile impacts the Dyneema ${ }^{\circledR}$ is indicated (red circles) and the slope of the $\delta_{\text {peak }}$ versus $t^{\prime}$ at that instant is denoted by $\dot{\delta}_{D}$.

Front face cutout targets: The ballistic resistance of the rear face of these targets in terms of the fraction of plies perforated versus impact velocity is considerably superior to the experiments of Karthikeyan et al. [2] as well as the corresponding laminate in the fully encased targets. This superior performance arises for two reasons:

(i) Similar to the fully encased targets, the rear Dyneema ${ }^{\circledR}$ face acquires some velocity prior to the impact of the projectile/ejecta.

(ii) As discussed in Sections 4.4.1 and 4.4.2, the projectile upon impact with the bare $\mathrm{Al}$ front face shatters, and it is this fragmented projectile along with any ejecta from the Al plate that impacts the rear Dyneema ${ }^{\circledR}$ face. The difference between the interaction of the intact projectile and the fragmented projectile with the Dyneema ${ }^{\circledR}$ laminate is sketched in Figure 4.19(b), where the 
fragmented projectile provides a more spatially distributed load on the Dyneema ${ }^{\circledR}$ face. The more distributed load will exert a lower interfacial pressure on the Dyneema ${ }^{\circledR}$ for the same impact velocity and thus the ballistic performance of the Dyneema ${ }^{\circledR}$ face is enhanced.

\subsubsection{Residual velocity of the projectile/ejecta}

The residual ejecta velocity $V_{r}$ exiting the rear laminates is plotted as a function of impact velocity $V_{i}^{*}$ in Figure 4.18(a). Data is only included from the fully encased and front face cutout targets of this study as (a) the ejecta from the front Dyneema ${ }^{\circledR}$ face then interacts with the $\mathrm{Al}$ plate making it hard to estimate $V_{r}$ exiting the Dyneema ${ }^{\circledR}$ plate and (b) data of $V_{r}$ is not available from the study of Karthikeyan et al. [2]. The data for $V_{r}$ in Figure 4.18(b) is obtained readily from Figure 4.6 by converting $V_{i}$ to $V_{i}{ }^{*}$ as detailed above. In both cases, just above the ballistic limit, the projectile exit velocity increases sharply and then proceeds to increase with increasing $V_{i}$ at an approximately linear rate (with slope greater than unity). The diminishing penetration resistance of a laminate impacted above its ballistic limit has been previously reported [10,11]. Interestingly, the residual velocity from the front face cutout target's rear laminate at a $V_{i}$ just above its higher $V_{b l}$ rises sharply to eventually meet the $V_{r}$ from the fully encased target at the same $V_{i}$. The reasons for this remain unclear.

\subsection{Concluding remarks}

We have designed four model targets to elucidate the perforation/penetration mechanisms in Dyneema ${ }^{\circledR}$ HB26 composites in a penetration resistant structure. The four 
targets were: (i) a bare Al plate; (ii) the aluminum plate fully encased in about a $6 \mathrm{~mm}$ thick casing of Dyneema ${ }^{\circledR}$; (iii) the fully encased plate with a portion of the Dyneema ${ }^{\circledR}$ removed from the front face so that the projectile impacts directly the Al plate and (iv) the fully encased plate with a portion of the Dyneema ${ }^{\circledR}$ removed from the rear face so that the projectile can exit the Al plate without again interacting with the Dyneema ${ }^{\circledR}$. The measurements show that the ballistic performance of these targets increases in the order: bare $\mathrm{Al}$ plate, rear face cutout plate, fully encased plates, and the front face cutout plate having the highest ballistic resistance. A combination of synchronized high speed photography (with three cameras) together with post-test examination of the target via high resolution X-ray tomography was used elucidate the deformation and perforation mechanisms and explain the above mentioned ballistic performance rankings. The primary mechanisms revealed by the analysis are as follows:

- $\quad$ Penetration of Dyneema ${ }^{\circledR}$ laminates supported on either a foundation or edge restrained occurs by the progressive fracture of fiber plies under the projectile. The number of fractured plies increased with impact velocity.

- The ballistic performance of Dyneema ${ }^{\circledR}$ laminates supported on a foundation is significantly lower (ballistic limit is lower by nearly a factor of two) compared to edge restrained plates.

- When Dyneema ${ }^{\circledR}$ laminates were pre-accelerated to an initial velocity prior to the impact of the projectile (in our case by the large radius of curvature bulge of the $\mathrm{Al}$ plate against the rear Dyneema ${ }^{\circledR}$ face), the apparent ballistic performance of the Dyneema ${ }^{\circledR}$ laminate was enhanced. This was due to a 
lowering of the relative velocity between the plate and the projectile (and thus the applied pressure) for a given projectile velocity.

- Fragmentation and concomitant spatial and temporal dispersion of the projectile by an intervening aluminum plate increased the ballistic limit of the laminate by distributing the interfacial forces between the projectile and laminate over a larger area, thereby reducing the contact pressure.

- $\quad$ Placement of the low impedance Dyneema ${ }^{\circledR}$ laminate on the front face of the targets delays the onset of projectile fragmentation. This resulted in targets with Dyneema ${ }^{\circledR}$ on their strike faces having a reduced ballistic performance at higher impact velocities.

Finally we conclude by noting that the simple model targets designed here have elucidated mechanisms that show how a fiber-reinforced polymer matrix composite can have both beneficial and detrimental effects on the ballistic resistance of targets. The suppression of the indirect tension mechanism by both pre-accelerating the laminate and dispersing the impact momentum provides an insight into how best to deploy a polymer matrix composite in a multi-material structure. A model target that uses these design parameters will be covered in Chapter 6. Lastly, a fuller understanding of these mechanisms is still needed, and particularly for the indirect tension mechanism, which is further explored in the following chapter.

\subsection{References}

[1] O'Masta MR, Deshpande VS, Wadley HNG. Mechanisms of Projectile Penetration in Dyneema Encapsulated Aluminum Structures. Int J Impact Eng 2014; In Press. 
[2] Karthikeyan K, Russell BP, Fleck NA, Wadley HNG, Deshpande VS. The effect of shear strength on the ballistic response of laminated composite plates. Eur J Mech ASolids 2013;42:35-53.

[3] Lesuer DR, Kay GJ, LeBlanc MM. Modeling Large-Strain, High-Rate Deformation in Metals. Livermore, CA: Lawrence Livermore National Laboratory; 2001.

[4] Wadley HNG, O'Masta MR, Dharmasena KP, Compton BG, Gamble EA, Zok FW. Effect of core topology on projectile penetration in hybrid aluminum/alumina sandwich structures. Int J Impact Eng 2013;62:99-113.

[5] Dehn JT. A unified theory of penetration. Aberdeen Proving Ground, MD: Ballistic Research Laboratory; 1986.

[6] Russell BP, Karthikeyan K, Deshpande VS, Fleck NA. The high strain rate response of Ultra High Molecular-weight Polyethylene: From fibre to laminate. Int J Impact Eng 2013;60:1-9.

[7] Phoenix SL, Porwal PK. A new membrane model for the ballistic impact response and V50 performance of multi-ply fibrous systems. Int J Solids Struct 2003;40:6723-65.

[8] Attwood JP, Khaderi S, Karthikeyan K, Fleck NA, O’Masta MR, Wadley HNG, et al. The out-of-plane compressive response of Dyneema composites. J Mech Phys Solids 2014:[Submitted for publication].

[9] Woodward RL, Egglestone GT, Baxter BJ, Challis K. Resistance to penetration and compression of fibre-reinforced composite materials. Compos Eng 1994;4:329-41.

[10] Shim VPW, Tan VBC, Tay TE. Modelling deformation and damage characteristics of woven fabric under small projectile impact. Int J Impact Eng 1995;16:585-605.

[11] Cunniff PM. An analysis of the system effects in woven fabrics under ballistic impact. Text Res J 1992;62:495-509. 


\section{Chapter 5. Defect Dependent Transverse Compressive Strength of Ballistic Grade Polyethylene Fiber Laminates ${ }^{8}$}

The impact study on the HB26 encased aluminum target (Chapter 4) showed the ballistic impact of a foundation supported $\left[0^{\circ} / 90^{\circ}\right]$ laminate results in penetration of the polymer composite even though transverse (out of plane) deflection of the laminate, with concomitant membrane stretching, is inhibited. The fibers break beneath the projectile, where only a strongly compressive force is being applied. The recent analysis by Atwood et al. [2] of the uniform compression of $\left[0^{\circ} / 90^{\circ}\right]$ laminates revealed a mechanism where the applied compressive stress loads plies in their fiber direction causing them to fail in tension. This indirect tension of fiber loading was previously introduced and explained to be due to a result of the high anisotropy in lateral (Poisson) strains transverse and parallel to the fiber direction of a unidirectional fiber reinforced ply (see Section 3.5), Figure 5.1(b).

\footnotetext{
${ }^{8}$ A paper [1] assembled from the work presented in this chapter has been submitted for publication to the International Journal of Solids and Structures.
} 


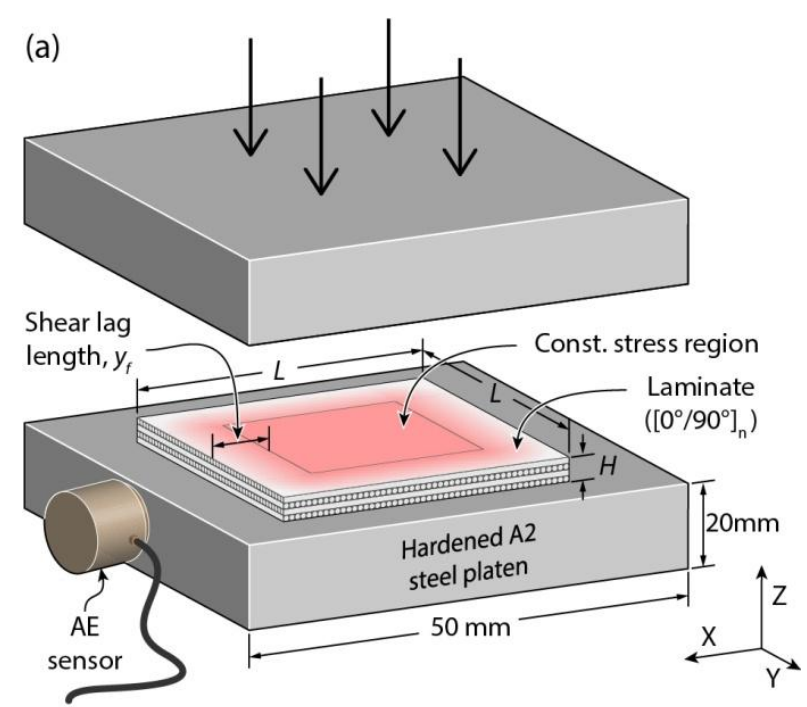

(b)

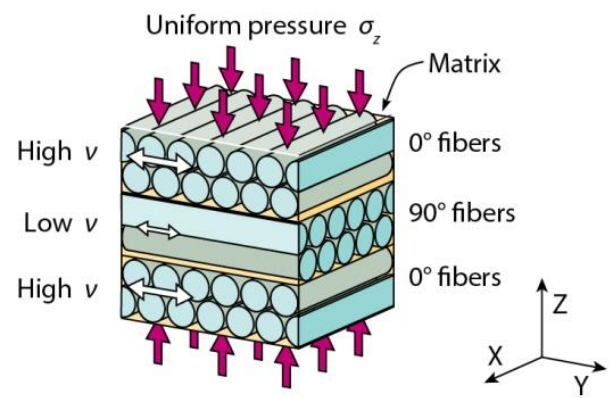

(c)

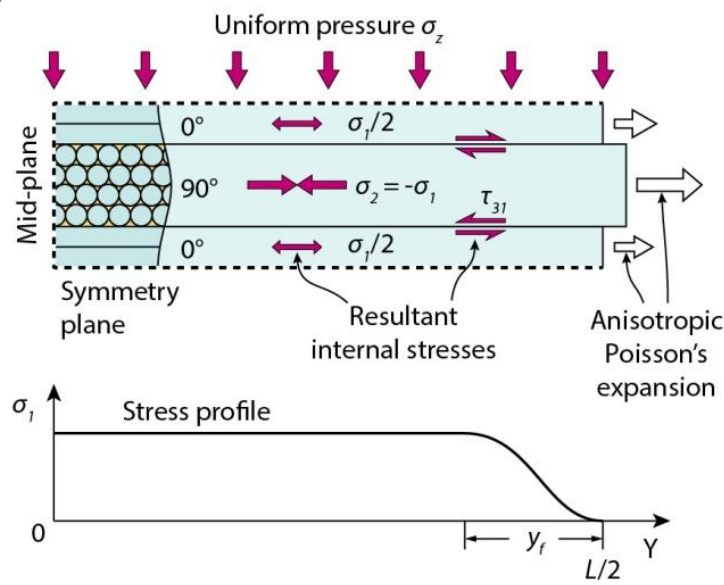

Figure 5.1. (a) Schematic illustration of an L square by $H$ thick sample under uniform compression. Compressive and resultant tensile stresses scale with the red color shading. (b) Schematic illustration of plies within a cross-ply laminate under a uniform compressive stress, $\sigma_{z}$. Poisson lateral expansion in the fiber direction is much less than that transverse to the fibers. (c) Schematic illustrations of the stress within the composite predicted by the shear lag model. 
When these unidirectional plies are stacked in a $\left[0^{\circ} / 90^{\circ}\right]_{\mathrm{n}}$ arrangement, the high lateral strain transverse to the fibers in one ply, loads the longitudinal fibers in the plies above and below in tension via a shear lag mechanism, Figure 5.1(c). When the tensile stress in a ply reaches the ply tensile strength, catastrophic ply failure (with large load drops under constant displacement rate loading) occurs. This failure event for a $\left[0^{\circ} / 90^{\circ}\right]_{80}$ laminate was capture by a high-speed camera (v.1610 Phantom, Vision Research Inc., Wayne, NJ) and an image sequence of the event is shown in Figure 5.2. It is notably different from the failure behavior observed when unidirectional laminates were uniformly compressed, Figure 3.10(b), and reveals the importance of lay-up for the activation of the indirect tension mechanism.
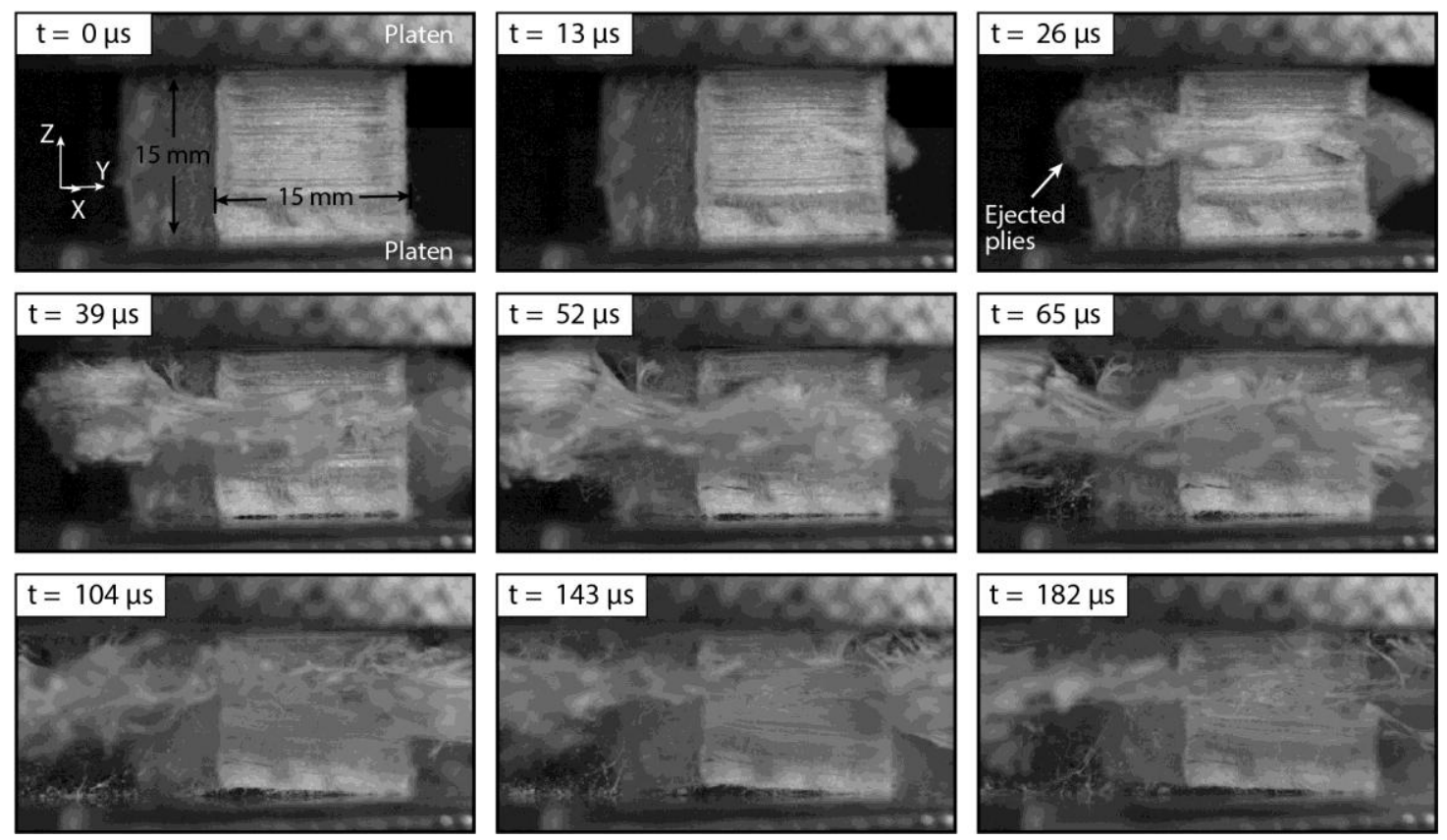

Figure 5.2. High-speed image sequence of the failure of a $\left[0^{\circ} / 90^{\circ}\right]_{80}$ laminate loaded in out of plane uniform compression. An exposure time $0.83 \mu$ s was used.

The model also predicts that this limiting condition for fiber failure can only be reached when the lateral dimensions of samples exceed twice the shear lag distance. The 
existence of the shear lag, a region of non-uniform stress transfer, has been observed with pressure sensitive film (layered grades of HS and HHS Fujifilm Prescale, Fujifilm Corp., Japan) placed between the sample and plate, Figure 5.3. The shear lag region leads to a predicted sample dimension dependence to the compressive strength that has been observed in experiments [2]. The model also predicts that reducing the thickness of the unidirectional ply, or increasing the matrix flow resistance also increases the compressive strength and supporting experimental evidence for this has also been reported [2]. A simplified form of the model is presented in Section 5.2.
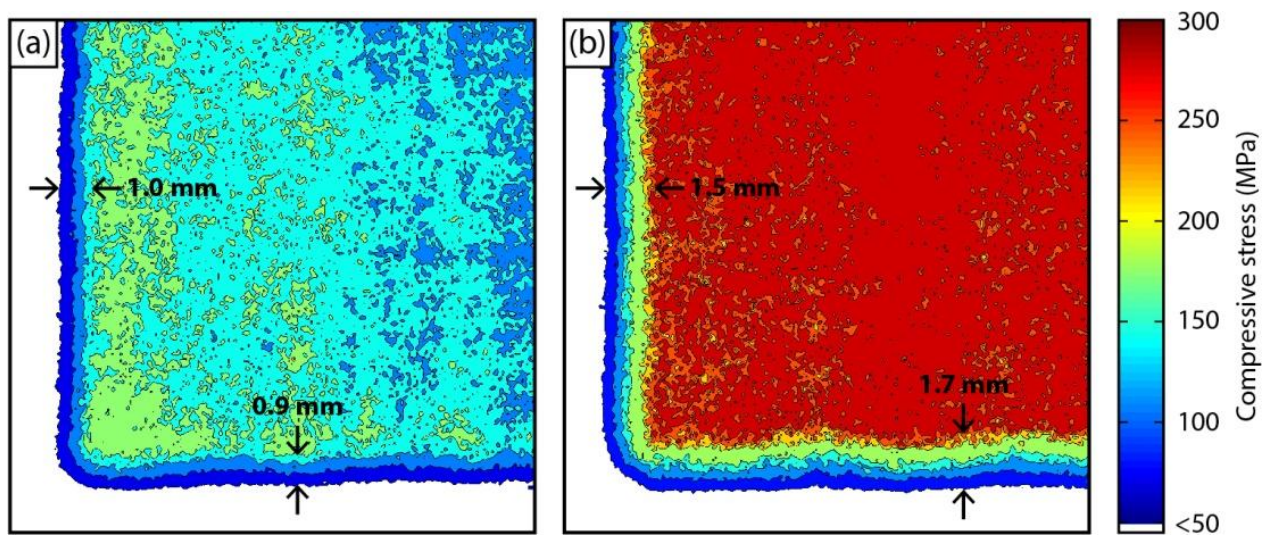

Figure 5.3. Contour plots of the pressure between a $10 \mathrm{~mm}$ thick $\times 30 \mathrm{~mm} \times 30 \mathrm{~mm}$ X131 sample and the loading platen after application of a nominal compressive stress of (a) $100 \mathrm{MPa}$ and $(b)$ $200 \mathrm{MPa}$. The length of the shear lag region is marked. For brevity, only a $15 \mathrm{~mm}$ corner section of the specimen is shown.

However, during experimental studies of the compression of UHMWPE composites conducted in parallel with the development of the indirect tension model, it became clear that both the compressive strength of laminates, and the variability in their strength depended upon the thickness a laminate. This feature of the compressive response is not predicted by the indirect tension model. Here we first investigate the through-thickness uniform compression response of UHMWPE fiber laminates as a function of both the in-plane sample dimension, $L$, and sample thickness, $H$. We find that 
thin laminates fail to reach their predicted strength, and link the strength reduction to increases in acoustic emission well before final (catastrophic) failure. We then use a variety of optical, ultrasonic and X-ray techniques to characterize these materials and identify the presence of a variety of defects. One of these is an elongated void that results from missing fibers, or groups of fibers, in a unidirectional ply. These findings prompted additional compression studies, including pressure distribution experiments, and tests using a bimodal ply thickness distribution. We show that missing fiber defects result in non-uniform loading of the samples, and a simple statistical model can be used to show that this is consistent with the measured thickness dependent compressive strength of UHMWPE fiber reinforced laminates.

\subsection{Materials and fabrication}

\subsubsection{Material Types}

This study focuses upon the compressive response, and characterizes the structure of, a representative grade HB26 Dyneema ${ }^{\circledR}$ UHMWPE fiber-polymer matrix composite, whose properties were discussed in Section 2.2. It is a non-woven composite, with a Dyneema ${ }^{\circledR}$ SK76 fiber volume fraction of $83 \%$, and utilizes a polyurethane resin for the matrix. To explore the generality of the phenomena discovered here, we also investigated the five other $\left[0^{\circ} / 90^{\circ}\right]$ UHMWPE fiber composite laminates introduced in Section 2.2. Table 2.2 tabulates the constituent materials, architecture and mechanical properties of all six laminate grades. 


\subsubsection{Laminate fabrication}

It will be shown below that all the tapes contained defects that extended a considerable distance in the fiber direction. To avoid stacking such defects close to each other in a laminate, $100 \mathrm{~mm}$ by $100 \mathrm{~mm}$ square sections of tape, with their sides in the fiber orientations, were cut from a $1.6 \mathrm{~m}$ by $1.6 \mathrm{~m}$ sheet of tape ${ }^{9}$ and stacked to preserve the $\left[0^{\circ} / 90^{\circ}\right]$ tape architecture in a specific sequence as follows. A $100 \mathrm{~mm}$ by $100 \mathrm{~mm}$ grid was drawn over the full area of the tape, and the bottom left corner segment labeled section $(1,1)$ was selected as the first tape section in the stack. The diagonal $(2,2)$ segment was used for the second layer, $(3,3)$ for the third and so on until segment $(15$, 15) had been added. Segment $(1,2)$ was then inserted and the diagonal sequence continued. The number of plies, $N(=2 \mathrm{n})$, needed to form various thickness $\left[0^{\circ} / 90^{\circ}\right]_{\mathrm{n}}$ laminates is tabulated in Table 5.1.

The stack of tapes was then placed in grade 1578 nylon bagging film (Fibre Glast Development Corp., OH, USA), that had been coated with grade 1153 FibRelease ${ }^{\circledR}$ release agent (Fibre Glast Development Corp.) and inserted between two $3 \mathrm{~mm}$ thick ground steel plates ${ }^{10}$. This pressing kit was then positioned between two platens that had been pre-heated to $100{ }^{\circ} \mathrm{C}$, and the high-pressure, warm compaction procedure outlined in Section 2.3.

\footnotetext{
${ }^{9}$ We note that the tapes contain a small number of fusion joints, resin splotches, and isolated patches of disarranged fibers on the surface. Care was taken not to sample these defects during laminate fabrication.

${ }^{10}$ The steel plate ensured a smooth pressed laminate surface, and the coated nylon film minimized laminate adhesion to the pressing surfaces.
} 
Table 5.1. Ply count to nominal thickness table for $\left[0^{\circ} / 90^{\circ}\right]$ n laminates.

\begin{tabular}{cccc}
\hline Laminate grade & $\begin{array}{c}\text { Thickness } \\
(\mathrm{mm})\end{array}$ & $\begin{array}{c}\text { Number of } \\
\text { plies, N }\end{array}$ & $\mathrm{n}$ \\
\hline HB26, HB50, & 1 & 16 & 8 \\
SR3124, & 5 & 80 & 40 \\
SR3136 & 10 & 160 & 80 \\
& 15 & 240 & 120 \\
\hline X106, X131 & 1 & 24 & 12 \\
& 5 & 120 & 60 \\
& 10 & 240 & 120 \\
\hline
\end{tabular}

Two techniques were used to cut smaller samples from the pressed laminates: i) Samples thinner than $2 \mathrm{~mm}$ were cut using either a sharp razor blade or a bench-top sheet metal shear; ii) thicker samples were cut on a band saw after the laminate had been clamped between two $1.5 \mathrm{~mm}$ thick sacrificial plates of aluminum.

\subsection{Review of the indirect tension model}

To aid in analyzing the results, this section presents a summary of Atwood et al.'s [2] analytical model for predicting the response of Dyneema ${ }^{\circledR}$ cross-ply laminates under uniform compression. The model is based on a ply's lateral strain anisotropy during through thickness compression, resulting in greater expansion strains transverse to the fibers than in the fiber direction (see the measurements in Sections 3.5 for substantiation), Figure 5.1(b). To maintain equilibrium between neighboring $0 \% / 90^{\circ}$ plies, a ply is placed in tension in the fiber orientation, with an equal compressive stress in the in-plane transverse direction, i.e. $\sigma_{22}=-\sigma_{11}$, Figure 5.1(c). A Tresca yield criterion is invoked that predicts yield will occur along the plane of maximum shear when the shear strength, $\tau$, is reached, i.e. $\tau=\left|\sigma_{1}-\sigma_{3}\right| / 2$, where the stresses $\sigma_{1}$ and $\sigma_{3}$ are the major and minor 
principal stresses. From Sections 3.5, we expect shear will occur along the plane normal to the fiber direction, i.e. the $\mathrm{x}_{2} \mathrm{x}_{3}$ plane, and thus $\sigma_{1}=\sigma_{22}$ and $\sigma_{3}=\sigma_{33}$. Furthermore the shear strength is assumed to linearly increase with $\mathrm{x}_{2} \mathrm{x}_{3}$ in-plane pressure, which is commonly observed in polymers and was verified in Section 3.4 at low pressures. The dependence is given as:

$$
\tau=\tau_{0}-\mu\left(\sigma_{22}+\sigma_{33}\right) / 2
$$

where $\tau_{0}$ is the shear yield strength in the absence of pressure and $\mu$ is the pressure sensitivity. Without directly measuring the shear strength at high pressures, the pressure sensitivity the parameter was used by Attwood et al. [2] as a fitting coefficient to the compressive strength data and has a value of near 0.05 .

The dependence of the out-of-plane transverse strain, $\varepsilon_{33}$, upon transverse stress, $\sigma_{33}$, can then be found to be given by:

$$
\varepsilon_{33}=\left\{\begin{aligned}
\frac{\sigma_{33}}{E_{m}}\left[1-\frac{v^{2} E_{f}}{E_{f}+E_{m}}\right], & 0 \geq \sigma_{33} \geq \sigma_{33}^{Y} \\
\frac{\sigma_{33}(1-\mu)+2 \tau_{0}}{E_{f}(1+\mu)}\left[1+2 \frac{E_{f}}{E_{m}} \frac{(1-v)}{(1-\mu)}\right]-\frac{2 \tau_{0}(1-v)}{E_{m}(1-\mu)}, & \text { otherwise }
\end{aligned}\right.
$$

where the transversely isotropic elastic constants of the ply are the Young's moduli in the fiber directions, $E_{f}$, the Young's moduli in the transverse directions, $E_{m}$, and the transverse Poisson's ratio $v=v_{23}$. We showed in Section 3.5 that the Poisson's expansion in longitudinal direction $v_{12}=v_{13}=0$, and assumed that no plastic deformation could occur in the fiber direction. The out of plane laminate yield strength is given by:

$$
\sigma_{33}^{Y}=-\frac{2 \tau_{0}}{1-\mu}\left[1+\frac{v}{\left(1+\frac{E_{m}}{E_{f}}\right)\left(\frac{1-\mu}{1+\mu}\right)-v}\right]
$$


When $\sigma_{11}$ equals the ply tensile strength, $\sigma_{f}$, the compressive peak strength, $\sigma_{i}^{\infty}$, (given as a positive value) is reached and given by:

$$
\sigma_{i}^{\infty}=\frac{1}{1-\mu}\left[\sigma_{f}(1+\mu)+2 \tau_{0}\right]
$$

For finite sized samples, a shear lag increases the stress from zero at the edge of the sample to a constant state of stress in the center of the sample, Figure 5.1(c). The shear lag length, $y_{s}$, was given by:

$$
y_{s}=h \frac{1-\mu}{2 \mu} \ln \left[1+\frac{\mu \sigma_{1}}{\tau_{0}}\right]
$$

where $h$ is the ply thickness. At tensile failure, the shear lag length, $y_{f}=$ $y_{s}\left(\sigma_{11}=\sigma_{f}\right)$, and the compressive peak strength (also given as a positive value) for a finite length sample is:

$$
\begin{gathered}
\sigma_{i}=\frac{1}{1-\mu}\left(1-\frac{2 y_{f}}{L}\right)^{2}\left[\sigma_{f}(1+\mu)+2 \tau_{0}\right] \\
-\frac{4 y_{f} \tau_{0}}{L \mu}\left(1-\frac{y_{f}}{L}\right)\left[\frac{h}{2 y_{f}} \frac{1+\mu}{\mu}\left(e^{a y_{f} / h}-1\right)-1\right]
\end{gathered}
$$

where $a=2 \mu /(1-\mu)$. It follows that $L \geq 2 y_{f}$ is the minimum sample length that will fail in tension.

\subsection{Transverse compressive strength}

Compression samples with in-plane dimensions of $L \times L$, were cut from laminated plates of thickness, $H$, Figure 5.1(a). Each sample was centered between two $50 \mathrm{~mm}$ long 
x $50 \mathrm{~mm}$ wide x $20 \mathrm{~mm}$ thick platens of A2 tool steel. The platens had been hardened to $64 \mathrm{HRC}$ and ground to an average surface roughness (Ra) of $0.5 \mu \mathrm{m}$. The platen face planarity was periodically tested using pressure sensitive films (grade HS Fujifilm Prescale, Fujifilm Corp., Japan), to confirm uniform contact across a sample. The sample/platen set-up was then centered between the load frame platens of a mechanical testing machine. For samples whose compressive failure force was less than $300 \mathrm{kN}$, an Instron (Norwood, MA) model 4208 equipped with either a $50 \mathrm{kN}$ or a $300 \mathrm{kN}$ load cell was used. For samples whose failure force exceeded this load, a Baldwin 1.33 MN universal testing machine was used. Duplicate samples were tested on each frame to ensure that they gave similar results. The samples were compressed at a displacement rate of $0.5 \mathrm{~mm} \mathrm{~min}^{-1}$. The displacement was measured using a laser extensometer with laser tags attached to the sample platens. Both the normal stress, $\sigma_{n}$, and strain, $\varepsilon_{n}$, were recorded, and the peak compressive nominal strength, $\sigma_{\max }$, determined for each sample. A minimum of five specimens were tested for each combination of material, sample length, $L$, and thickness, $H$.

During each compression test, acoustic emission (AE) was continuously monitored to detect stress waves that were generated by abrupt material failure processes [3]. The thin sample size, and explosive nature of failure, precluded attachment of $\mathrm{AE}$ transducers directly to the sample. Instead a (model R15 $\alpha$, Physical Acoustics Corp; Princeton Jct., NJ) $150 \mathrm{kHz}$ resonant piezoelectric transducer was attached to the side of the bottom sample platen with Dow Corning high vacuum grease couplant, Figure 5.1(a). A model 1220 2/4/6 pre-amplifier, with $20 \mathrm{kHz}$ high-pass filter, amplified the signal by $20 \mathrm{~dB}$. A PCI-2 18-bit A/D data acquisition system with an effective band-pass filter of 
$20 \mathrm{kHz}$ to $400 \mathrm{kHz}$ was then used to record the pre-amplified signal at a sampling rate of $1 \mathrm{MHz}$. The PAC AE-win acquisition software then analyzed the signal to identify discrete events. As a compromise between capturing new acoustic events and minimizing the recording of a previous event's reverberation as a new event, the event duration and lockout time parameters were set to $1200 \mu$ s and $600 \mu$ s respectively. Since tensile UHMWPE fiber failure has been shown to be a high amplitude (>80dB) event compared to other failure mechanisms (matrix deformation, fiber-matrix debonding and delamination) [4,5], the event threshold was set at $78 \mathrm{~dB}$ to avoid non-fiber failure related events. 
(a) HB26

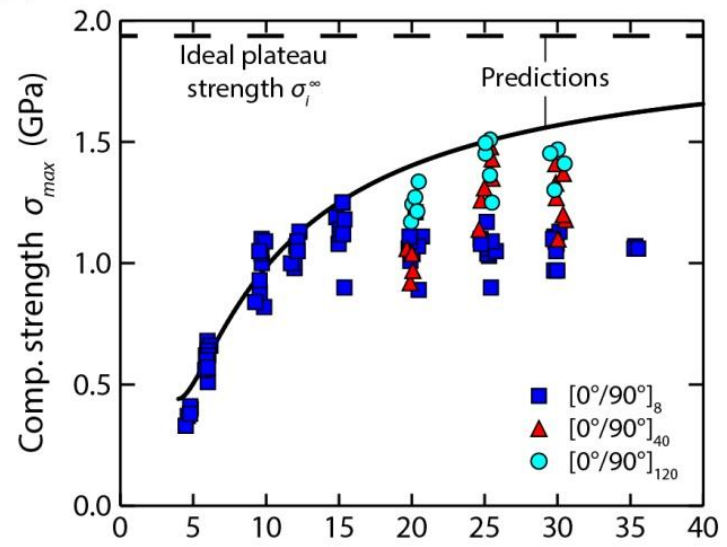

(c) $\mathrm{X} 106$

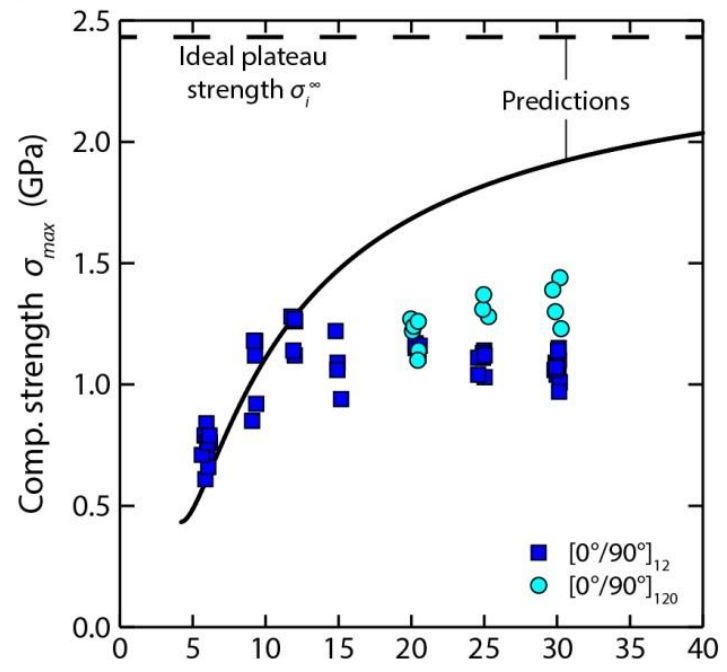

(e) SR3124

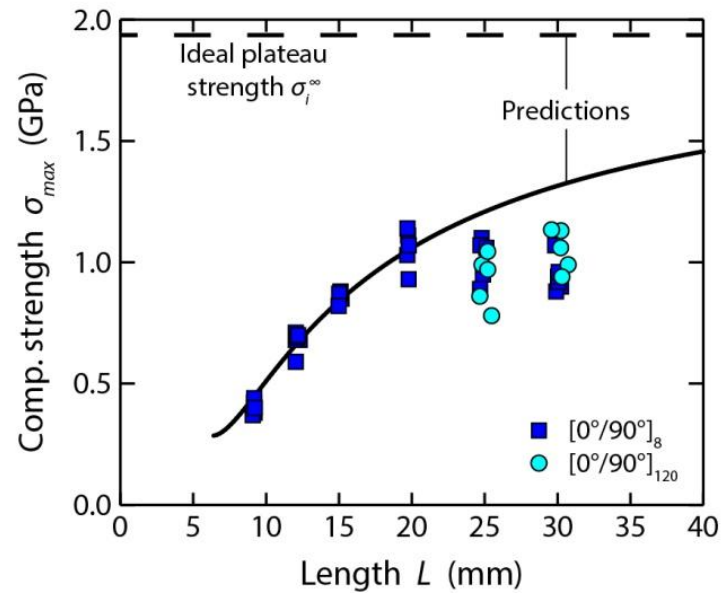

(b) HB50

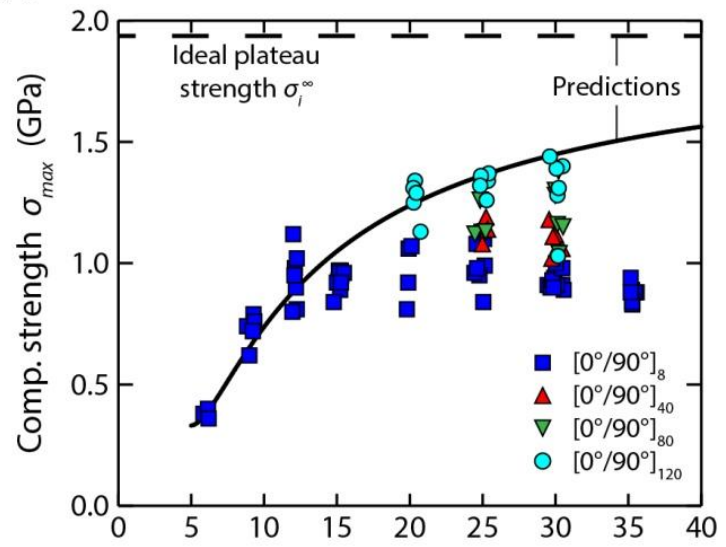

(d) $\mathrm{X} 131$

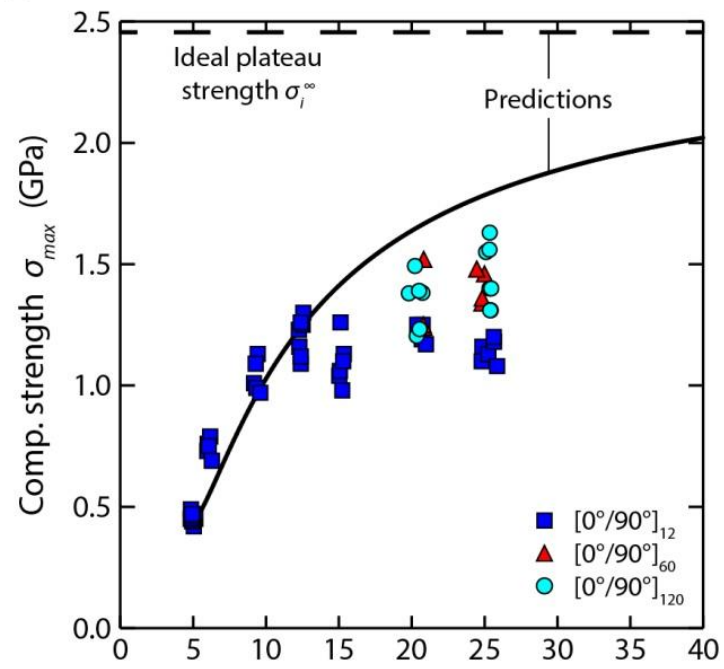

(f) SR3136

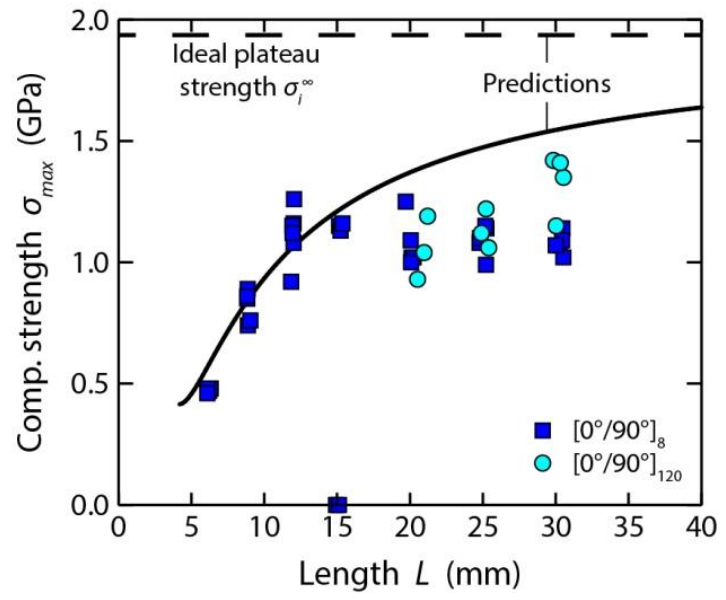

Figure 5.4. Transverse compression strength, $\sigma_{\max }$, plotted as a function of sample length, $L$, for all laminate grades. Scatter is seen in the measured strength as well as significant deviation from predicted values for thinner laminates at large coupon sizes. 
The through thickness compressive strength, $\sigma_{\max }$, of 16 ply $\left[0^{\circ} / 90^{\circ}\right]_{8} \mathrm{HB} 26$ laminates are plotted as a function of sample length $L$ in Figure 5.4(a). Each dark blue square represents a single tested sample. The solid black line is the predicted compressive strength using Eq. ( 5-6) using the ply values summarized in Table 2.2. ${ }^{11}$ The dashed black line is the predicted plateau compressive strength, $\sigma_{i}^{\infty}$, for an infinitely large sample using Eq. ( 5-4). The measured values compare well with the predictions for samples starting with lengths near the shear lag limit $\left(L>2 y_{f} \approx 5 \mathrm{~mm}\right.$ as given by Eq. ( 5-5)) and extending to $L=15 \mathrm{~mm}$. However, the strengths of larger samples tended to plateau $\sigma_{\text {max }} \approx 1.1 \mathrm{GPa}$, which is much lower than the prediction $\sigma_{i}^{\infty}=1.9 \mathrm{GPa}$.

The transverse compressive strength results for 16 ply $\left[0^{\circ} / 90^{\circ}\right]_{8} \mathrm{HB} 50$ laminates (dark blue squares) are shown in Figure 5.4(b). For this more compliant matrix laminate, the data are again consistent with the model predicted increase in strength with sample size, but again the experimental data fall below the predicted strength for samples with $L$ $>15 \mathrm{~mm}$. Similar trends were also apparent for the thinner ply, and smaller diameter fiber Dyneema ${ }^{\circledR}$ grades X106 and X131, Figure 5.4(c) and (d), and for the Honeywell Spectra ${ }^{\circledR}$ laminates SR3124 and SR3136, Figure 5.4(e) and (f). The results of additional experiments with thicker $\left[0^{\circ} / 90^{\circ}\right]_{\mathrm{n}}$ laminates with $\mathrm{n}=12,40,80,120$ and 240 are shown on Figure 5.4 and indicate that the maximum strength approached model predictions as the laminate thickness increased.

Typical nominal stress-strain responses of three $L=12 \mathrm{~mm}, 16$ ply HB26 samples are shown in Figure 5.5(a). For the first sample (shown in black), the stress, $\sigma_{n}$,

\footnotetext{
${ }^{11}$ For the predictions, the value of the pressure sensitivity coefficient $\mu$ was fit to the $H=1 \mathrm{~mm}, L \leq 15$ $\mathrm{mm}$ data set. The same value of $\mu$ was used for the HB26, HB50, SR3124 and SR3136 and slightly depressed values were used for X106 and X131, as shown in Table 1.
} 
monotonically increased with strain, to a strain, $\varepsilon_{n} \approx 0.2$. The sample catastrophically failed at a nominal stress, $\sigma_{\max }=1.13 \mathrm{GPa}$. Failure was highly energetic with ejection of fractured plies from the edges of the sample, and a very loud single AE event, with an amplitude of $115 \mathrm{~dB}$. Atwood et al. [2] reported a similar loading response and catastrophic failure mode. The second sample, shown by the green curve, followed a similar loading path as the first, but failed at a lower stress of $1.05 \mathrm{GPa}$. A smaller $89 \mathrm{~dB}$ AE event was released just prior to the catastrophic $116 \mathrm{~dB}$ event. The third sample, shown in red, had three distinct $>114 \mathrm{~dB}$ amplitude AE events with two occurring almost simultaneously and coinciding with a drop in stress at $\sigma_{n}=0.86 \mathrm{GPa}$. The sample stress then recovered, but only reached a value of $\sigma_{\max }=0.88 \mathrm{GPa}$ before catastrophic failure and release of a third, and final, AE event. Given the very high amplitude (>78dB) of these AE events, we associate them with local tensile failure of plies, and the rapid redistribution of stress to surrounding un-failed material. Furthermore, AE events with amplitudes $>110 \mathrm{~dB}$ coincided with a detectable drop in nominal stress. 
(a)

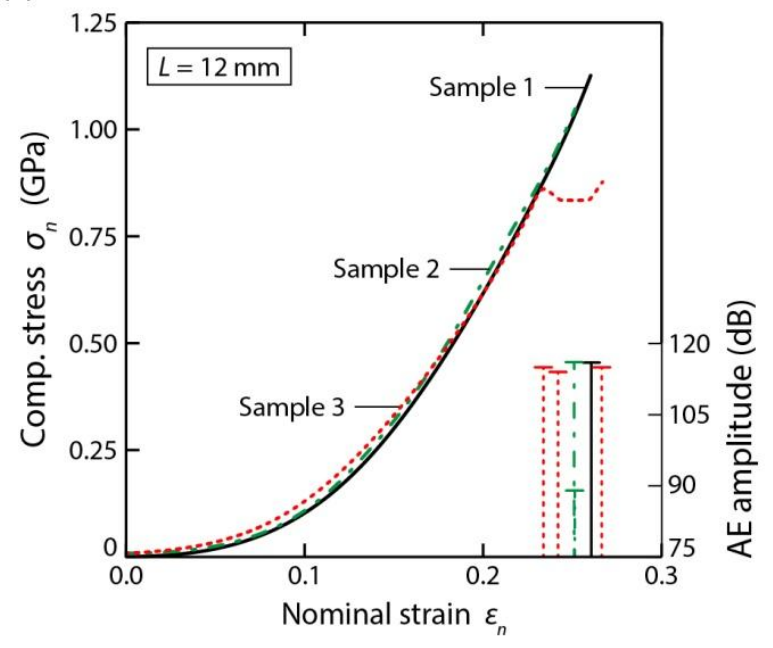

(b)
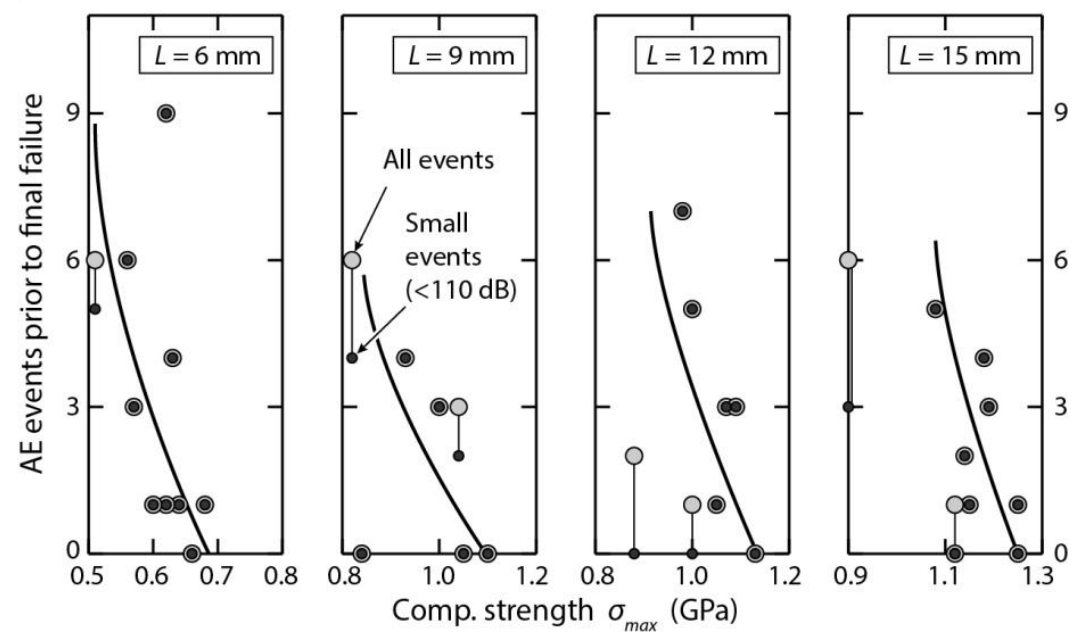

Figure 5.5. The nominal transverse compressive stress, $\sigma_{n}$, and the amplitude of acoustic emission (AE) events are plotted against nominal strain, $\varepsilon_{n}$, for three $\mathrm{L}=12 \mathrm{~mm}\left[0^{\circ} / 90^{\circ}\right]_{8} \mathrm{HB} 26$ samples. (b) The compressive strength, $\sigma_{\max }$, of $\left[0^{\circ} / 90^{\circ}\right]_{8} \mathrm{HB} 26$ samples are plotted as a function of the number of $A E$ events.

In Figure 5.5(b) we plot for each sample both the total number of AE events prior to final failure (light grey circles), and the number of smaller amplitude events $<110 \mathrm{~dB}$ (dark grey circles) prior to final failure as functions of compressive strength, $\sigma_{\max }$; the number of large events $(\geq 110 \mathrm{~dB})$ is therefore the difference between these two data points. For the $L=12 \mathrm{~mm}$ size samples, those reaching the highest recorded strength $\sigma_{\max }$ $=1.13 \mathrm{GPa}$ released no AE emissions prior to final failure. However, as the strength 
decreased, the total number AE events increased. Furthermore, the sample with the lowest strength, $\sigma_{\max }=0.87 \mathrm{GPa}$, released only two AE events prior to final failure, but both registered above $110 \mathrm{~dB}$. Similar results are seen for sample sizes of $L=6,9$, and $15 \mathrm{~mm}$. Hence, low strength values are correlated with increased numbers of AE events, with large amplitude events being especially detrimental to peak strength.

The number of AE events prior to final failure for 240 ply thick, HB26, HB50, $\mathrm{X} 106$ and X131 grade laminates is shown as a function of compressive strength in, Figure 5.6. In general, the number of AE events is again correlated with a reduction in laminate transverse compressive strength. It is also evident that the number of AE events for the experimental grades, X106 and X131, was greater than for the commercial grades, HB26 and HB50, Figure 5.6(a). Furthermore, both experimental grades registered more numerous large ( $\geq 110 \mathrm{~dB}$ ) AE events, while only two lower strength HB26 samples, and none of the HB50 samples, registered any, Figure 5.6(b). Of the two experimental grades, $\mathrm{X} 131$ registered more large, as well as total, AE events, and X131 which never attained its predicted strength, Figure 5.4(c), always had two or more large AE events prior to final failure. 


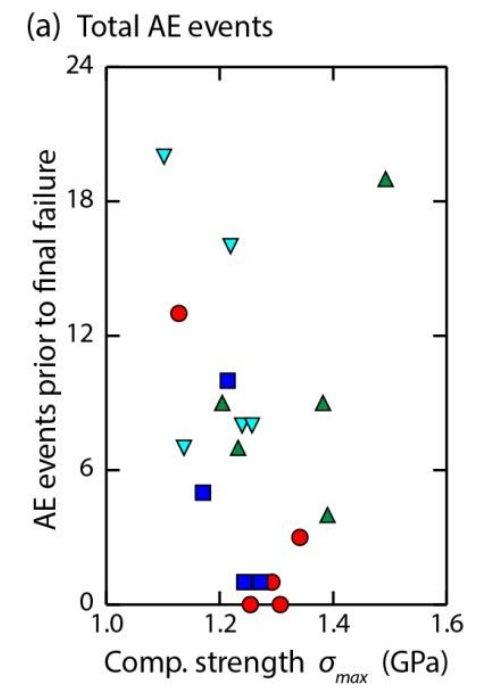

(b) Large ( $\geq 110 \mathrm{~dB}) \mathrm{AE}$ events

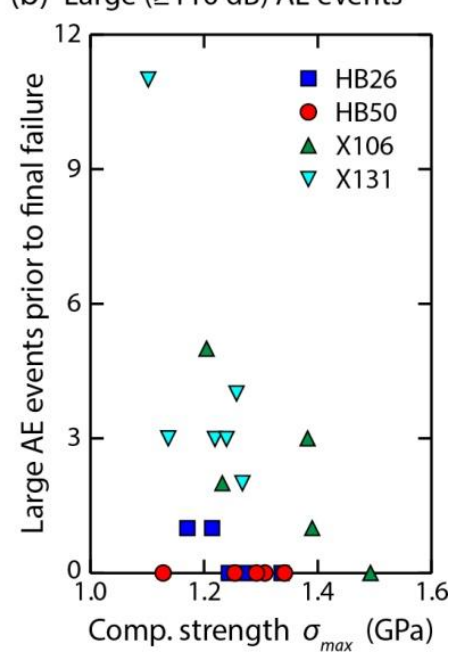

Figure 5.6. Plots of (a) the total AE events prior to final failure and (b) large AE events $\geq 110 \mathrm{~dB}$ prior to final failure as functions of compressive strength, $\sigma_{\max }$, for $L=20 \mathrm{~mm}$, thick $\left[0^{\circ} / 90^{\circ}\right]_{120}$ laminates.

\subsection{Defect characterization}

Optical imaging: A cross hatch pattern of light and dark contrast can usually be observed upon visual inspection of UHMWPE fiber reinforced tape. The first defect visualization method therefore digitally imaged the transmission of light through the thickness of a $170 \mathrm{~mm} \times 170 \mathrm{~mm}$ laminated plate. Both thin $\left[0^{\circ} / 90^{\circ}\right]_{8}$ and much thicker $\left[0^{\circ} / 90^{\circ}\right]_{60}$ laminates were prepared for each material grade. Each plate was positioned in front of a diffuse white light source, and the central $150 \mathrm{~mm}$ x $150 \mathrm{~mm}$ region was photographed with a Nikon D7000 DSLR camera mounted with a Micro-Nikkor 105 mm f/2.8 lens. Using Adobe Photoshop, the white and black levels of the digital optical transmission image were adjusted to enhance the contrast.

A photograph taken with optical transmission through a $\left[0^{\circ} / 90^{\circ}\right]_{8} \mathrm{HB} 26$ laminate is shown in Figure 5.7(a). Numerous light and dark bands are aligned with the $\mathrm{X}$ - and $\mathrm{Y}$ fiber directions, and typically span the entire $150 \mathrm{~mm}$ x $150 \mathrm{~mm}$ image window. Similar 
observations can be seen in the other $\left[0^{\circ} / 90^{\circ}\right]_{8}$ laminates, Figure $5.7(\mathrm{~b}-\mathrm{f})$. The bands are present in the smaller diameter fiber X106 and X131 laminates, but their width is much smaller making them more difficult to observe, Figure 5.7(c) and (d). The Spectra Shield $^{\circledR}$ laminates SR3124 and SR3136, Figure 5.7(e) and (f), are qualitatively similar to HB50 and HB26 counterparts. It is also evident that the crossing of two orthogonal light grey bands, results in an intersection of similar shade. However, the crossing of two darker grey bands results in a darker grey intersection, consistent with the notion that the dark streaks contain defects that scatter light. 
(a) HB26

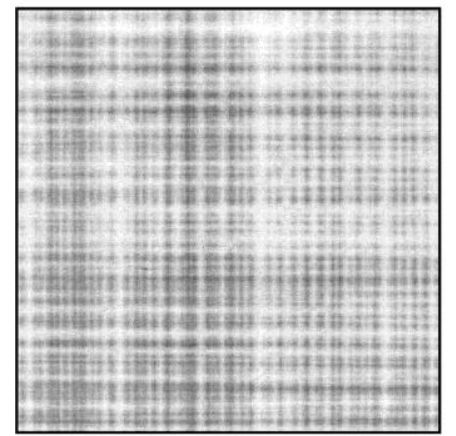

(c) $\mathrm{X} 106$

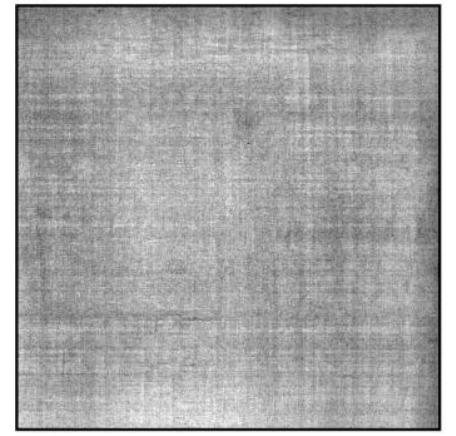

(e) SR3124

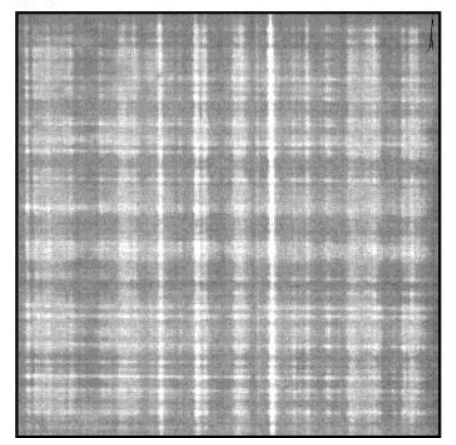

(b) HB50

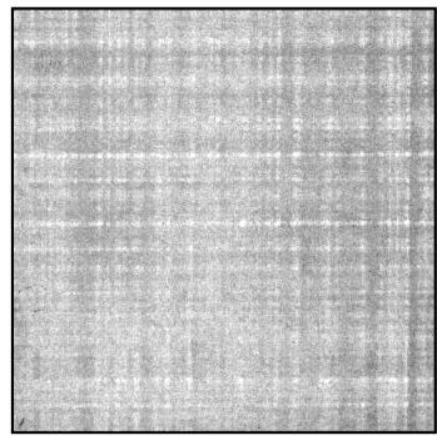

(d) $\mathrm{X} 131$

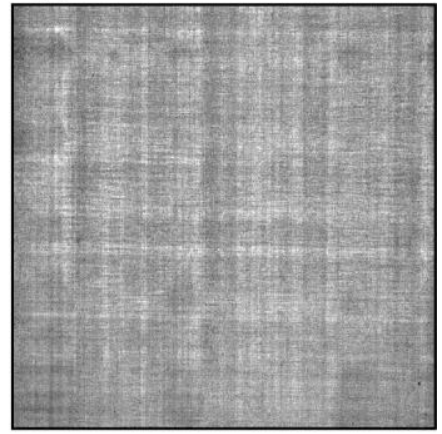

(f) SR3136

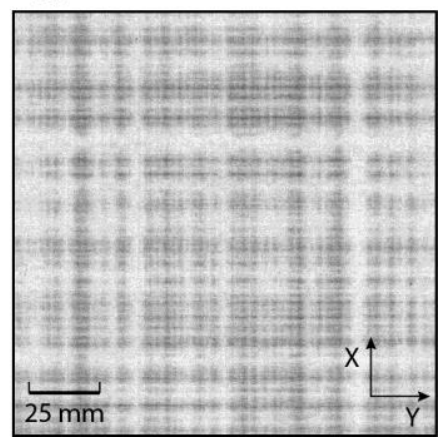

Figure 5.7. Optical backlit photographs of $\left[0^{\circ} / 90^{\circ}\right]_{8}$ laminated plates of UHMWPE fiber laminates.

The optical transmission of thicker, 120 ply laminates was also investigated,

Figure 5.8. The images also contain printed brand and logo information which was distributed widely across the tapes from which the samples were constructed. The light and dark contrast bands are still clearly present, but compared with the 16 ply HB26 laminate, Figure 5.7(a), the number of bands and their level of contrast are reduced, 
Figure 5.8(a). Similar observations apply to the other five grades shown in Figure 5.8(bf), and their 16 ply counterparts, Figure 5.7(b-f).

(a) HB26

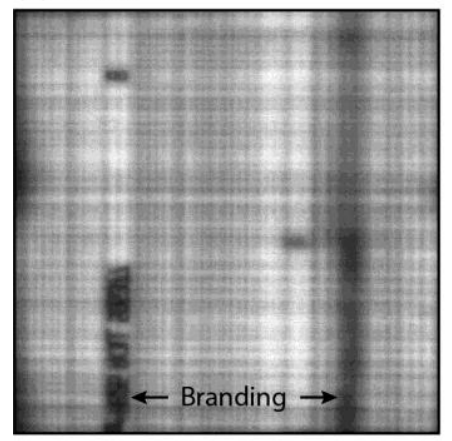

(c) $\mathrm{X} 106$

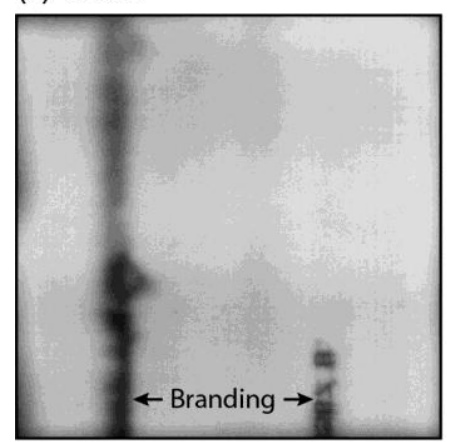

(e) SR3124

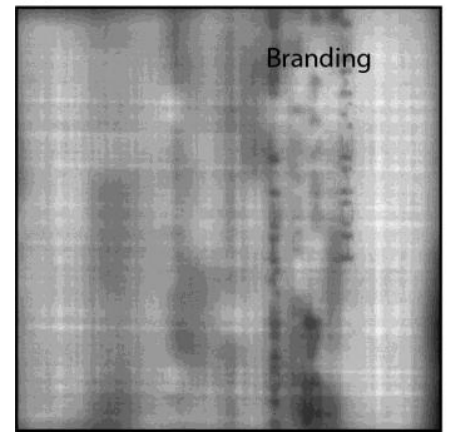

(b) HB50

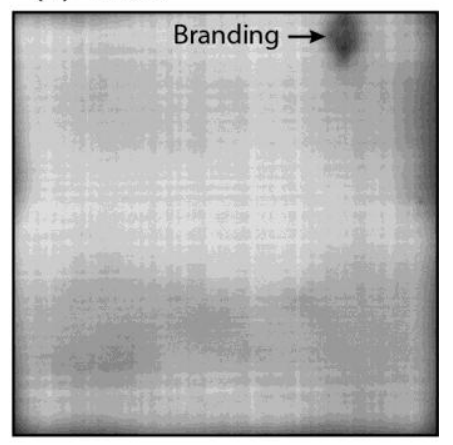

(d) $\mathrm{X} 131$

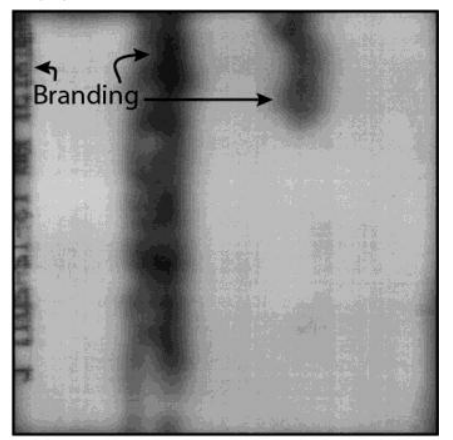

(f) SR3136

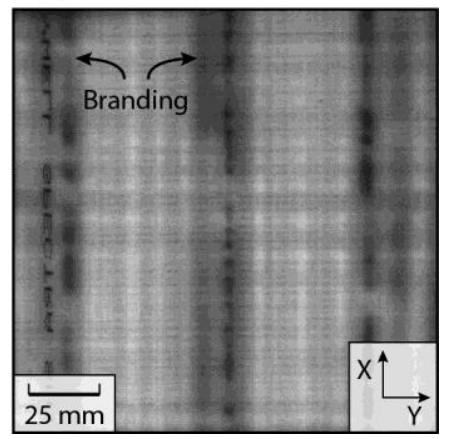

Figure 5.8. Optical transmission photographs of $\left[0^{\circ} / 90^{\circ}\right]_{60}$ laminated plates. The manufacture's printed brand identifiers are marked.

Ultrasonic C-scan: The thinner, $\left[0^{\circ} / 90^{\circ}\right]_{8}, 170 \mathrm{~mm} \times 170 \mathrm{~mm}$ plates used for optical transmission imaging were also examined with ultrasonic attenuation $\mathrm{C}$-scan imaging. Normally an ultrasonic couplant, such as water, is used to reduce the acoustic impedance mismatch (and surface reflection coefficient) between the medium supporting 
the incident signal and the sample. However, the ingress of the couplant into surface opening defects within the sample can occur, and reduce their signal attenuation contribution. Instead an air-coupled non-contact ultrasonic inspection technique was performed using facilities at QMI, Inc. (Huntington Beach, CA, USA). The tests were conducted with a QMI Sondal 007CX Airscan instrument, using a pair of $225 \mathrm{kHz}$ Airscan transducers. The receiver's gain and attenuation settings were adjusted to change the average received transmission signal to $80 \%$ of the receiver's saturation limit. The relative transmitted amplitude was recorded as the sample was translated in $1.016 \mathrm{~mm}$ steps over the $\mathrm{X}-\mathrm{Y}$ plane. The amplitude data is then presented as an image at the same magnification and sample orientation used for optical transmission characterization.

An ultrasonic C-scan of the same area of the16 ply HB26 laminate examined optically (Figure 5.7(a)) is shown Figure 5.9(a). The light and dark contrast bands running the full length and width of the sample are again present, and in the same locations as the optical image. However, the lower spatial resolution of the ultrasonic image has led to an increase in the width of the bands, and some narrow bands were not resolved. Ultrasonic C-scan images of the 16 ply HB50, X131 and SR3136 laminates are shown in Figure 5.9(b-d) and are similar to the HB26 sample. It is concluded that the defects responsible for attenuating light transmission also attenuate the propagation of ultrasonic pulses through the thickness of the laminate. Similar highly elongated $(>150$ $\mathrm{mm})$ and narrow $(<5 \mathrm{~mm})$ defects have been reported in other studies of these materials using ultrasonic transmission and terahertz radiation imaging [6,7]. 
(a) HB26

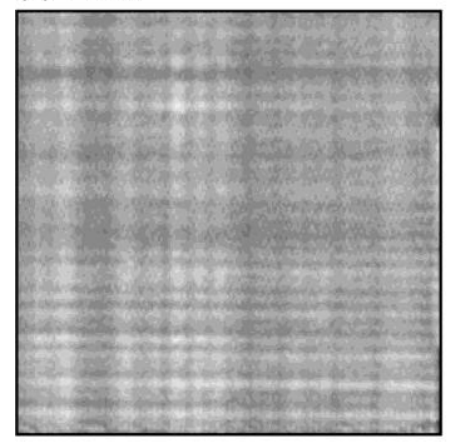

(c) $\mathrm{X} 131$

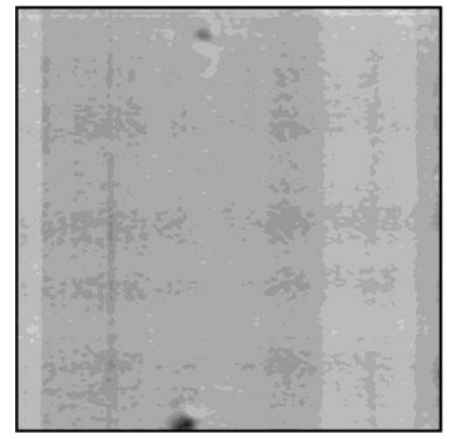

(b) HB50

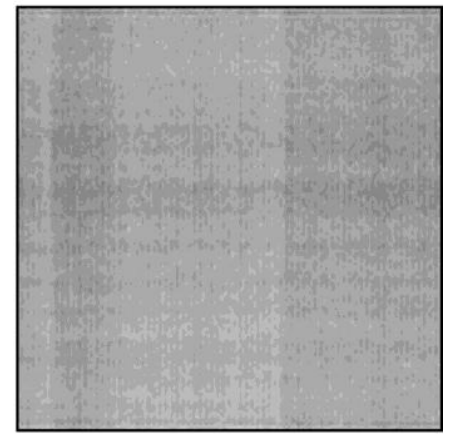

(d) SR3136

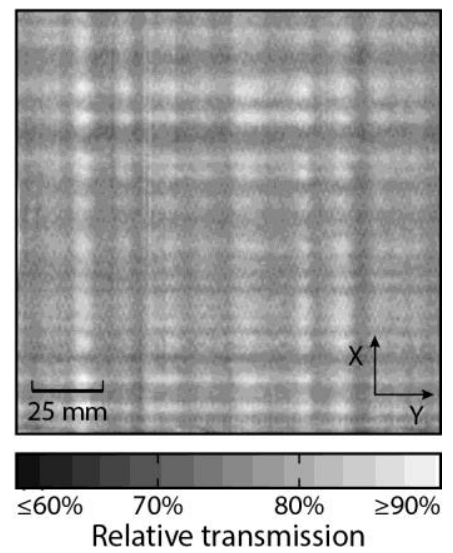

Figure 5.9. Ultrasonic transmission amplitude contour maps of the same $\left[0^{\circ} / 90^{\circ}\right]_{8} \mathrm{UHMWPE}$ laminates shown in Figure 5.7.

Micro x-ray computed tomography: To gain insight into the origin of the contrast variations, we conducted $\mu \mathrm{XCT}$ scans of low and high light (and ultrasound) attenuating regions in a 16 ply HB26 laminate. This technique images local changes in a materials density. We used an Xradia (Pleasanton, CA, USA) model $\mu$ XCT-200, and rotated samples while collecting a series of $2 \mathrm{D}$ x-ray radiographs which were then reconstructed to obtain a three-dimensional image of the sample. The instrument was equipped with a 10x magnification lens, allowing analysis of a $1.3 \mathrm{~mm}$ diameter by $1.3 \mathrm{~mm}$ long cylindrical volume with a voxel size of $1.365 \mu \mathrm{m}$. Samples $8 \mathrm{~mm}$ x $8 \mathrm{~mm}$ in dimension were cut from $\left[0^{\circ} / 90^{\circ}\right]_{8}$ plates at an orientation of $\pm 45^{\circ}$ to the plies. Each sample was placed between the x-ray source and detector, with its plies oriented $\pm 45^{\circ}$ to the axis of 
rotation ${ }^{12}$. The sample was rotated in $0.06^{\circ}$ steps from $-92^{\circ}$ to $92^{\circ}$ (at $0^{\circ}$, the plate normal was aligned with the x-ray path), and the series of x-ray projections reconstructed using Xradia's TXM Reconstructor software package. The computed volume was then visualized and analyzed with Avizo Fire v. 7 (FEI Visualization Sciences Group) software.

A YZ-plane (laminate cross-section) tomogram of a low attenuation region is shown in Figure 5.10(a). The small diameter darker grey circles in the $0^{\circ}$ plies are fibers running in and out of the plane of the image. The lighter grey regions located between the fibers is the matrix. The fibers in the $90^{\circ}$ plies were more difficult to visualize, in part because they meander in and out of the observation plane.

\footnotetext{
${ }^{12}$ Practice revealed improved reconstruction results with smaller samples and resolution of the fibers in both $0^{\circ}$ and $90^{\circ}$ plies by angling of the plies in relation to the $\mathrm{x}$-ray path.
} 
(a) Low attenuation region: $Y Z$ plane

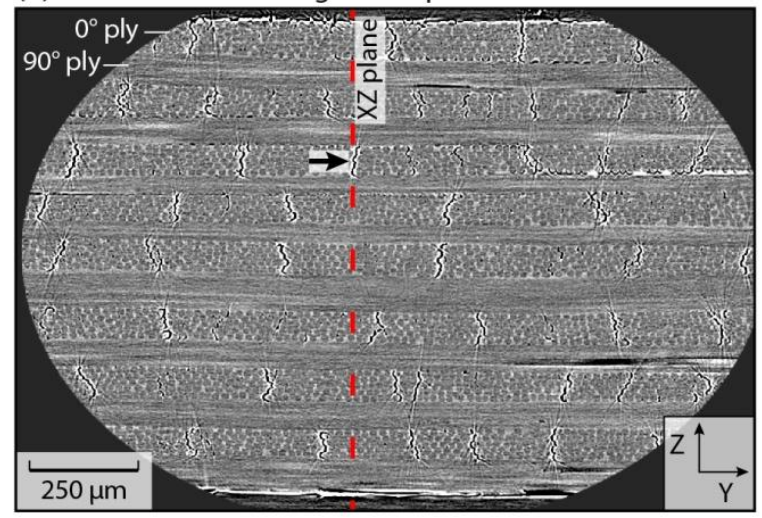

(b) Low attenuation region: $X Z$ plane

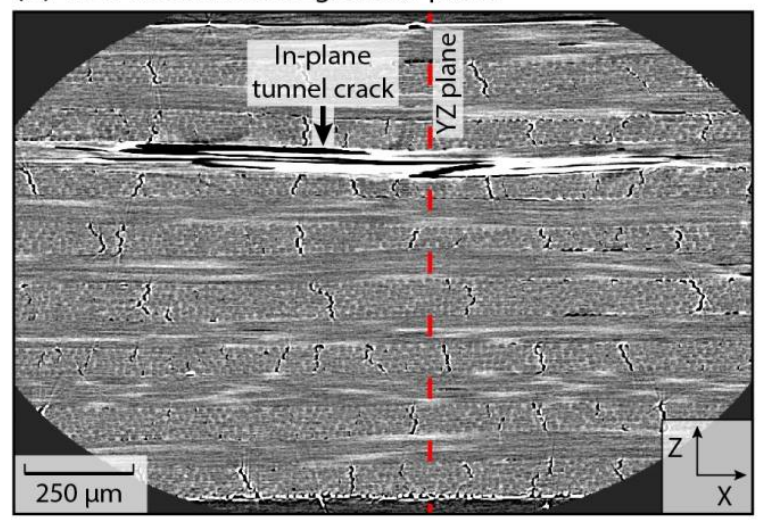

(c) Low attenuation region: 3D view

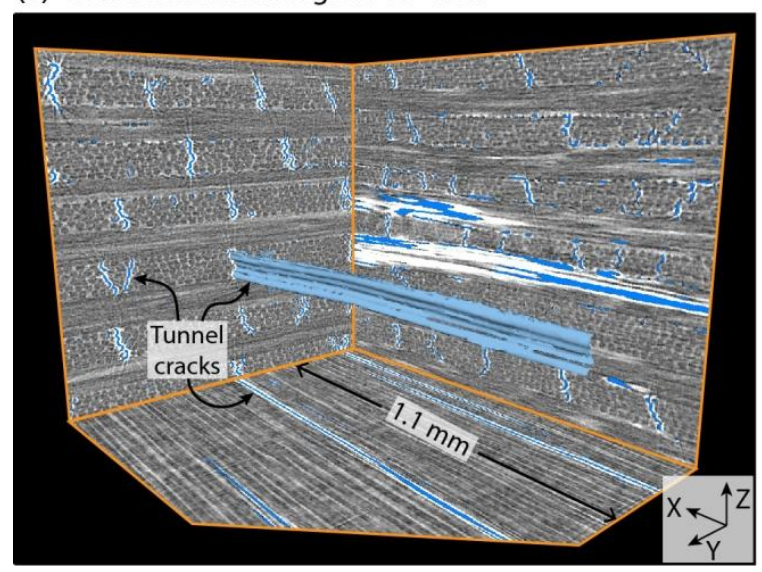

(d) High attenuation region: YZ plane

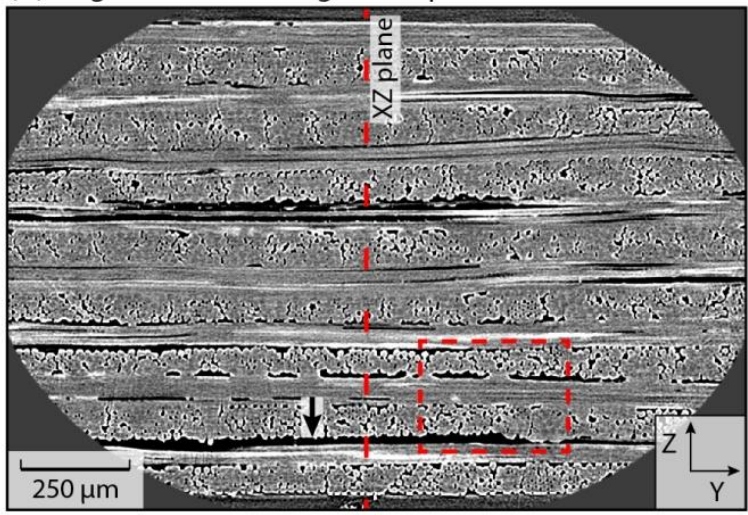

(e) High attenuation region: $X Z$ plane

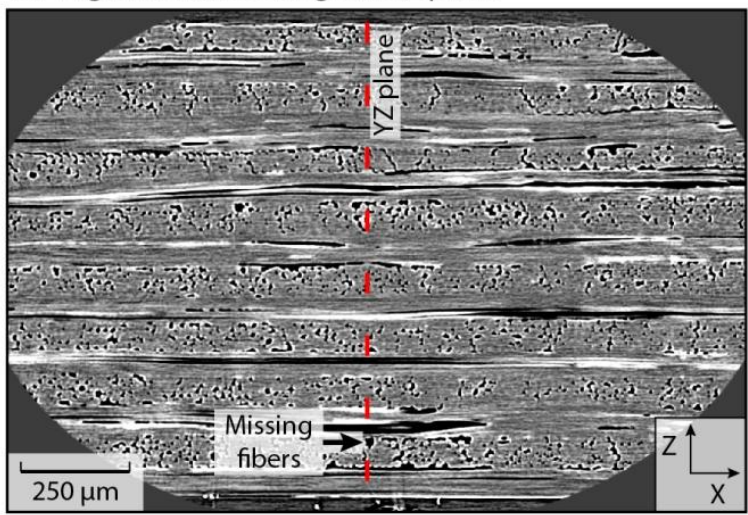

(f) High attenuation region: magnifaction of $d$

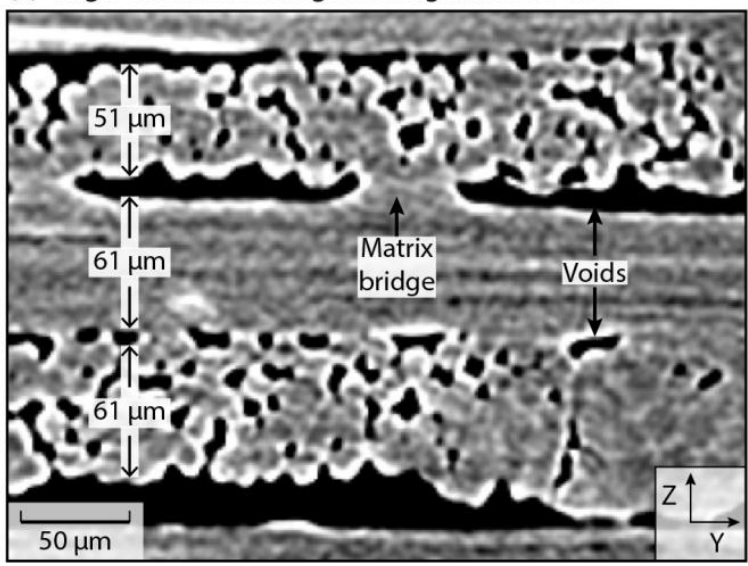

Figure 5.10. MicroXCT orthogonal cross-sections of a $\left[0^{\circ} / 90^{\circ}\right]_{8} \mathrm{HB} 26$ laminate through a region of low optical attenuation $(a-c)$ and a region of high attenuation $(d-f)$.

The low attenuation of the laminate contained periodically distributed, vertically

oriented (Z-direction) cracks. One such crack is identified by an arrow. An orthogonal

cross-section image through this crack is shown in Figure 5.10(b). The crack in this 
image appears as a region of black and white pixels, which is representative of no material (a void) and a scanning artifact, respectively. Figure 5.10(c) shows a threedimensional view of three orthogonal planes, where the voids have been isolated using the procedure described in Appendix $\mathrm{C}$ and rendered blue. One of the cracks propagating in the X-direction has been resolved in 3D. It is apparent the crack is only a fraction of a fiber diameter in crack opening displacement. These cracks resulted in a voided region volume fraction of about $3 \%$ existing throughout the composite. We identify these flaws as tunnel cracks $[8,9]$ that form upon cooling after consolidation, as a result of the substantial anisotropy in thermal expansion coefficient of a ply $[10,11]$. The negative coefficient of thermal expansion in the fiber direction results in thermal elongation of the ply during cooling while the substantial positive coefficient of thermal expansion in the transverse directions (combined with a very low transverse tensile strength of the ply) results in thermal contraction (and fracture) upon cooling. This transverse contraction is impeded by the plies above and below, leading to the array of tunnel cracks.

A $\mu \mathrm{XCT}$ cross-sectional reconstruction of a high attenuating region is shown in Figure 5.10(d). While tunnel cracks are still present, the region has a higher concentration of voids (about 10 vol.\%) and a different type of defect that is elongated in the Ydirection and located at the interface of two plies ( its location is identified by an arrow). An orthogonal cross-section through the elongated void is shown in Figure 5.10(e). The two cross-section images are consistent with a cylindrically shaped void whose long axis is parallel with the fiber direction in a ply. An absent fiber and insufficient matrix flow during consolidation would form such a void. It is not a delamination between plies as Figure 5.10(d) may have otherwise suggested. A magnified image of the region marked 
by a square box in Figure 5.10(d) is shown in Figure 5.10(f). Several of oval-shaped voids can be seen in two $0^{\circ}$ plies. The plies appear poorly compacted rather than delaminated, because they are locally thinner than the average ply thickness $h=67 \mu \mathrm{m}$. From these observations it is concluded that plies have regions of missing fibers that result in long fiber-oriented voids. These voids provide optical and ultrasonic scattering surfaces, and their presence is therefore consistent with the source of the defects observed in previous optical and ultrasonic images.

It is clear from the $\mu \mathrm{XCT}$ observations that voids at different ply interfaces through a laminates thickness can sometimes overlap. To quantify this, the X-ray tomograms have been analyzed for the HB26 laminate and a histogram of the total thickness of voids summed through the laminate's thickness (in the Z-direction) is plotted in Figure 5.11 for both low- and highly-attenuating regions shown in Figure 5.10. The total void thickness is binned in increments of $6.825 \mu \mathrm{m}$. The relative frequency was then determined from the area occupied within a binned thickness range, normalized by the total area analyzed in the X -Y plane. For a low-attenuating region, the average total void thickness was $26 \pm 18 \mu \mathrm{m}$, Figure 5.11(a). In high-attenuation regions, the total void thickness was approximately normally distributed, with an average thickness of $100 \pm$ $26 \mu \mathrm{m}$, Figure 5.11(c). Since a 16 ply HB26 laminate is about $1 \mathrm{~mm}$ thick, Figure 5.11(b), about $10 \%$ of the laminate's thickness consisted of voids in high attenuation regions, but only $3 \%$ of the thickness consisted of tunnel crack type voids in a lowattenuation region. Additionally, the total thickness of voids for both sampled volumes was substantially greater than the variance in laminate thickness, Figure 5.11(b) and (d). 
(a) Low-attenuation region: void thickness

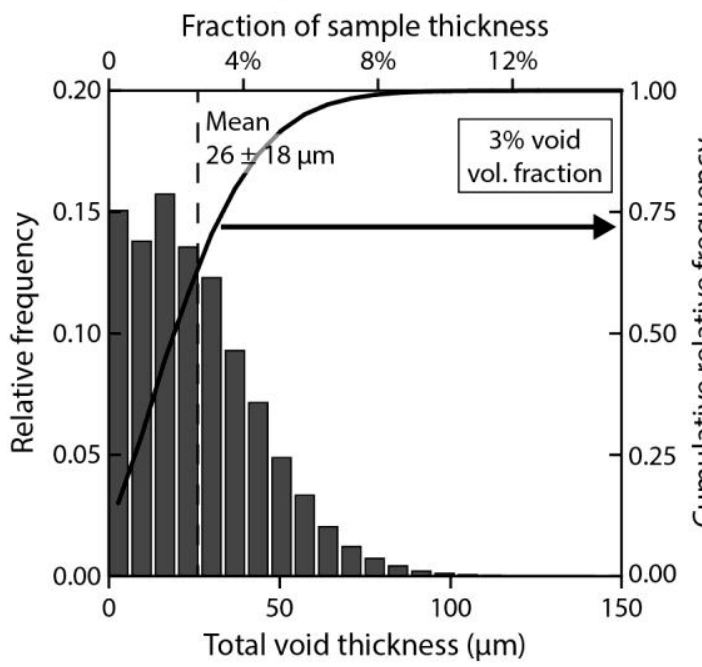

(b) Low-attenuation region: laminate thickness

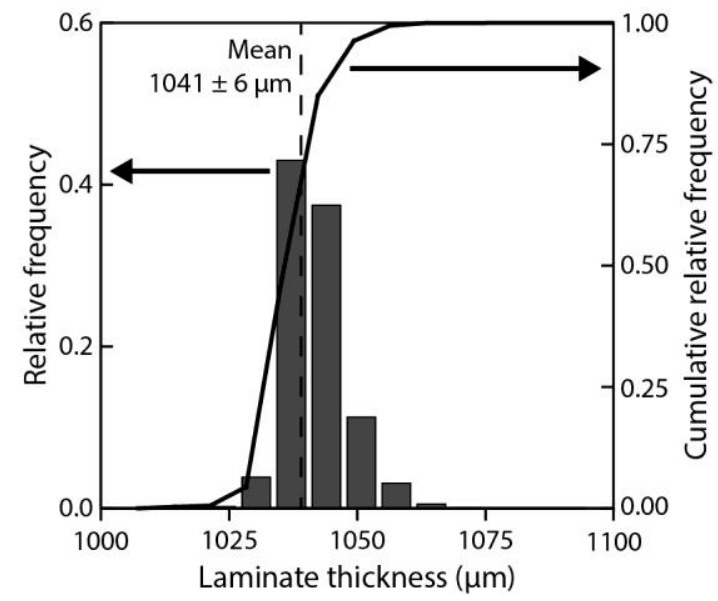

(c) High-attenuation region: void thickness

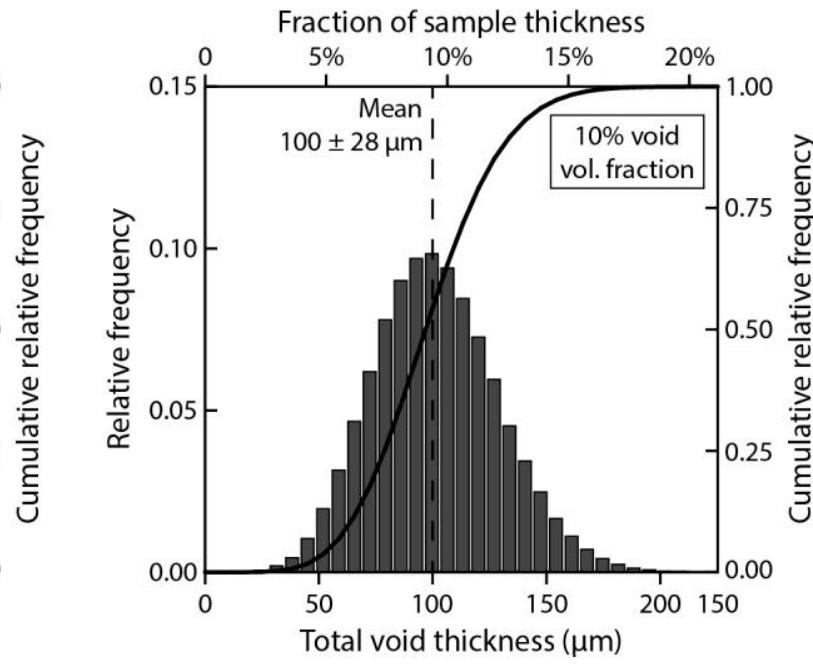

(d) High-attenuation region: laminate thickness

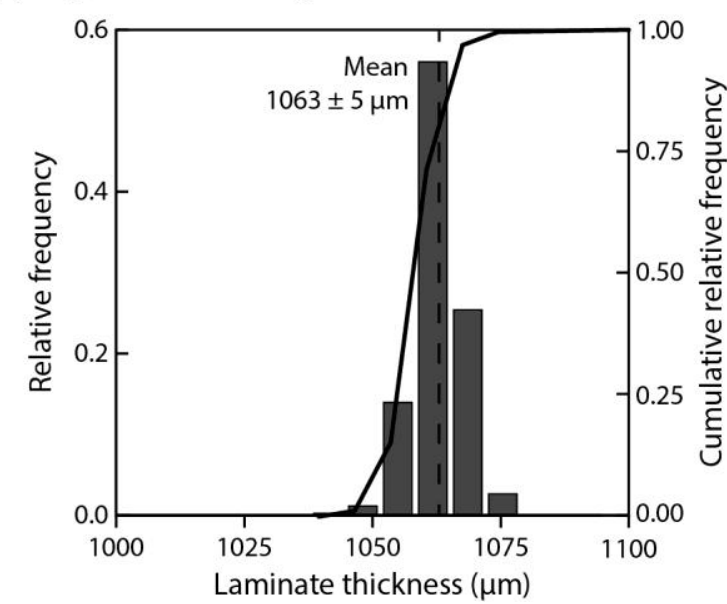

Figure 5.11. Histograms of the total void thickness and laminate thickness for regions of low optical attenuation (in Figure 5.10) are shown in (a) and (b). (c) and (d) show analogous data for high attenuation regions.

To investigate the origin of the high attenuation region voids, we have examined the as-received $\mathrm{HB} 26\left[0^{\circ} / 90^{\circ}\right]_{2}$ tape prior to its consolidation. A backlit optical image of such a tape is shown in Figure 5.12(a). The cross-hatched light and darker contrast bands can be clearly seen. An x-ray radiograph of the region marked by the square box in Figure 5.12(a) is shown in Figure 5.12(b). The bright white cross-hatching aligns with the dark contrast bands in the Figure 5.12(a). In-plane cross-sectional $\mu \mathrm{XCT}$ reconstructions of the same area are shown in Figure 5.12(c) and (d) and reveal the prominent white lines 
in Figure 5.12(b) to be phase contrast artifact located at material-void interfaces. The large void identified by the arrow in Figure 5.12(c) is shown to correspond to a missing group of $\sim 8$ fibers ( 2 fibers thick and 4 fibers wide) in Figure 5.12(e) and (f). Thus the origin of the missing fibers, and subsequent voids in the laminates, is traceable to missing fiber defects in the tape. Furthermore, the defects create variability in the ply thickness, and, while hot-pressing of the tape causes some rearrangement of fibers to fill some of the missing fiber voids, the volume of voids remains quite large in the conventionally pressed laminate. Of further note, the location of voids seems equally as probable in the internal two plies as the external two, further confirming that the defects arise during fabrication of the unidirectional plies. 
(a) Optical backlit image

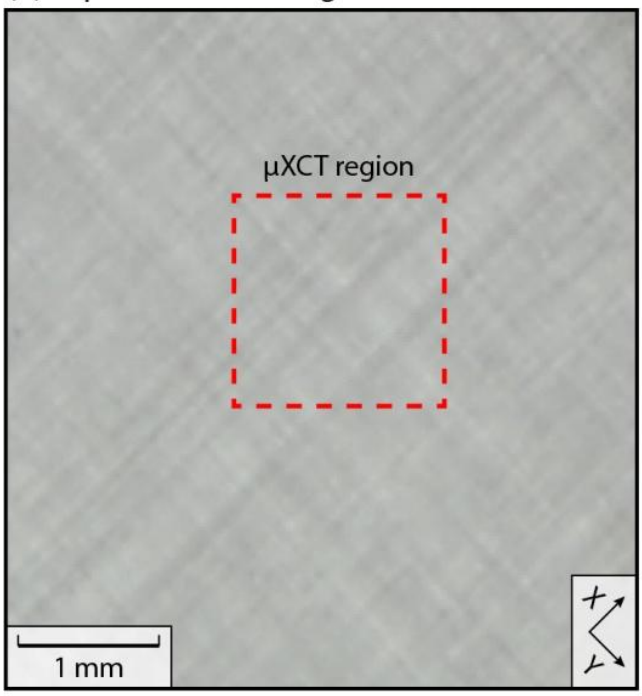

(c) $\mu \mathrm{XCT}$ section: $\mathrm{XY}$ plane $(\mathrm{Z}=45 \mu \mathrm{m})$

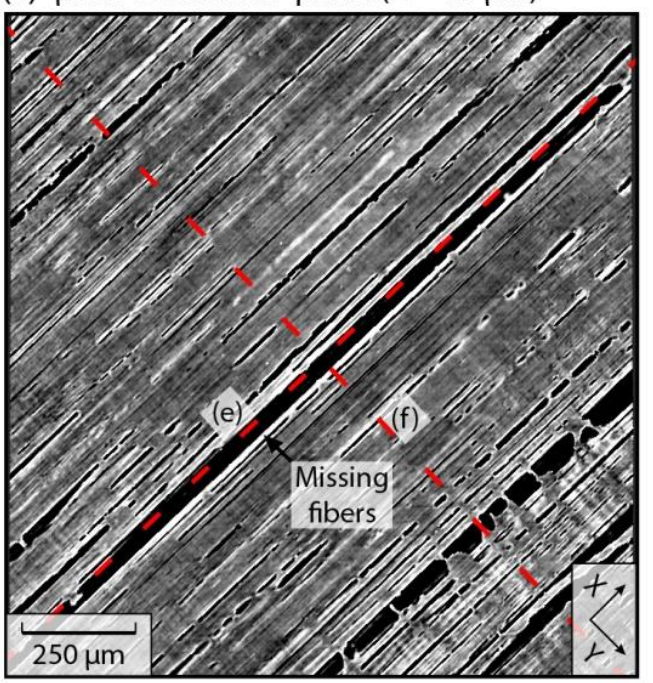

(b) X-ray radiograph

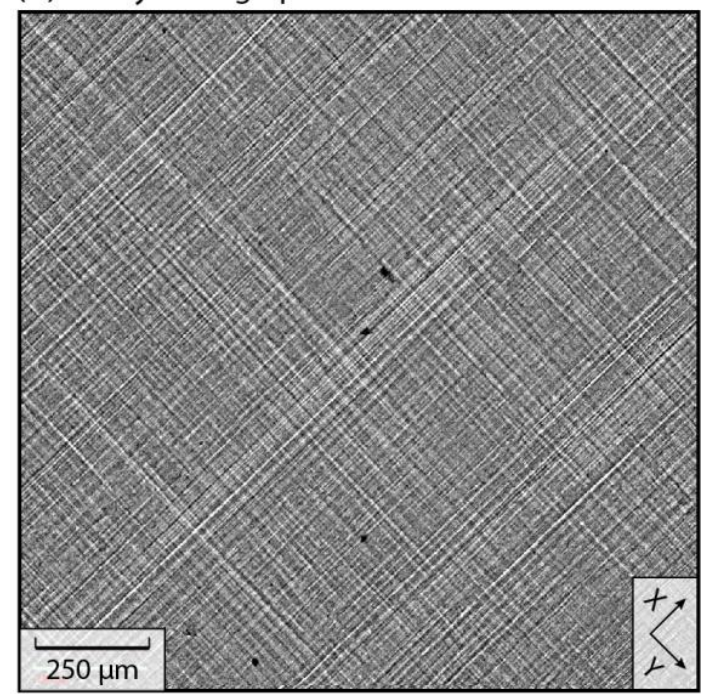

(d) $\mu X C T$ section: $X Y$ plane $(Z=215 \mu \mathrm{m})$

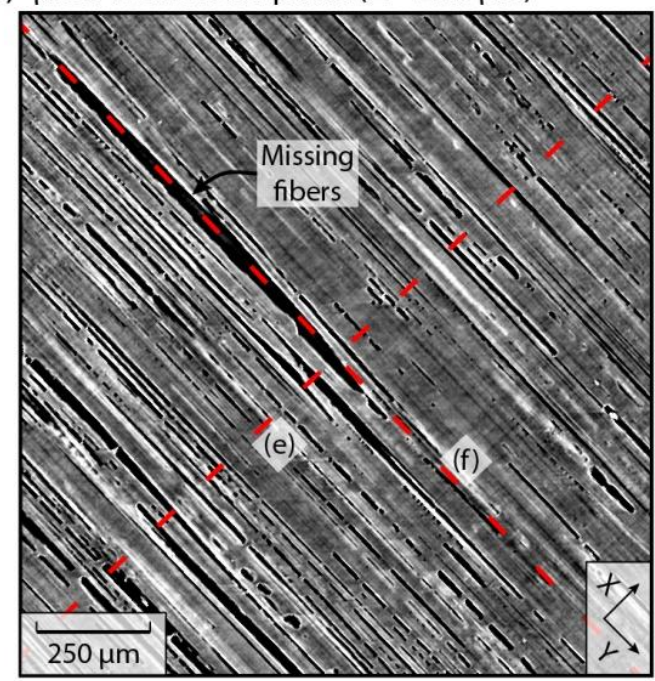

(e) $\mu X C T$ reconstruction: XZ plane

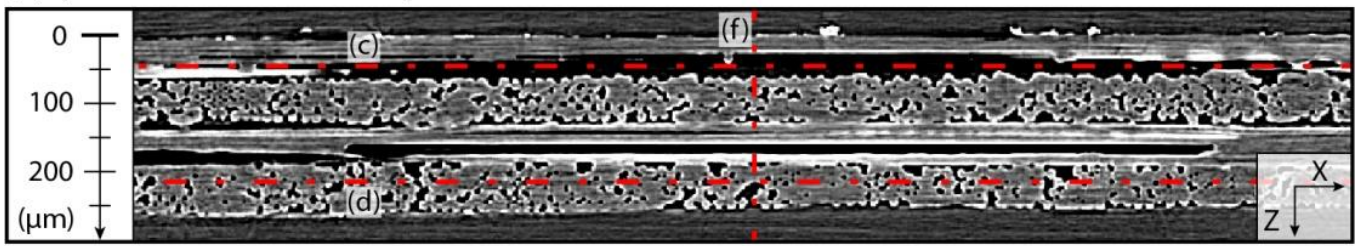

(f) $\mu \mathrm{XCT}$ reconstruction: $Y Z$ plane

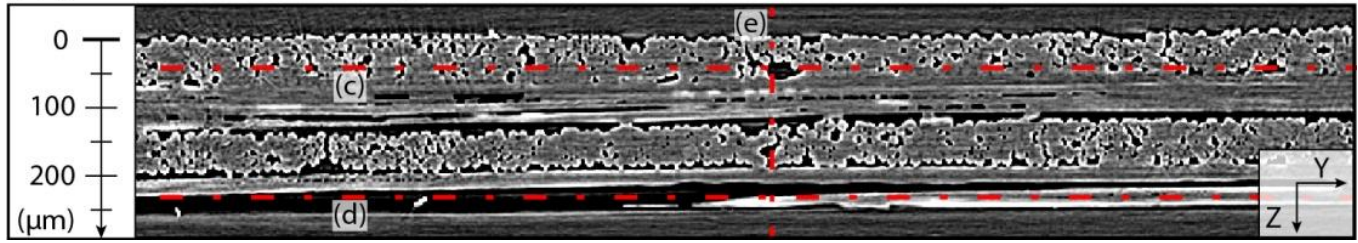

Figure 5.12. (a) Backlit optical transmission image of an unconsolidated HB26 tape. (b) A radiograph of the region highlighted in part a. (c-f) Reconstructed $\mu X C T$ orthogonal crosssections of the region highlighted in part a. 


\subsection{Pressure distribution, tunnel crack healing and ply thickness distributions}

To explore how the presence of missing fiber defects affected mechanical behavior, we first investigated the distribution of pressure over a laminate's loading area. We used grade HS, Fujifilm Prescale pressure sensitive film ${ }^{13}$ to record local pressures between 50 and $200 \mathrm{MPa}$ at the surface of a compressed laminate. The film was placed between a 16 ply $L=30 \mathrm{~mm}$ HB26 laminate and the test rig compression platen. An optical transmission photograph of the laminate's central $27 \mathrm{~mm} \times 27 \mathrm{~mm}$ region is shown in Figure 5.13(a). The sample was then loaded to $\sigma_{n}=100 \mathrm{MPa}$, held for 20 seconds and unloaded. Figure 5.13(b) shows a pressure map for the central $27 \mathrm{~mm} \times 27$ mm region.

\footnotetext{
${ }^{13}$ Red ink is released from microcapsules at a rate dependent upon the local pressure. The intensity of the pressure is proportional to the intensity of the color. After exposure, the film was scanned using an Epson Perfection V500 Photo Scanner at a resolution of 2400 dpi. The pixel intensity of values of the green channel values were compared to a calibration curve to create a pressure map.
} 
(a) Backlit photograph

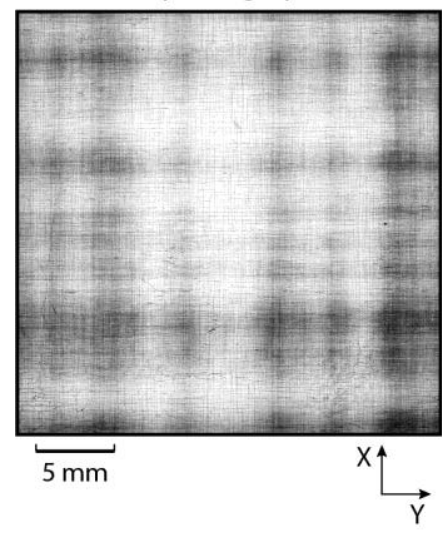

(d) Sim. backlit image

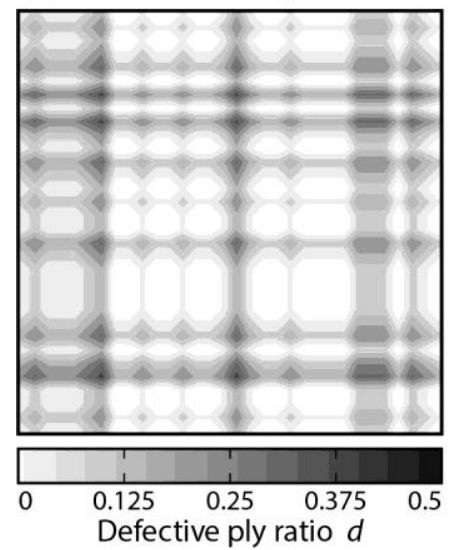

(b) Pressure map, $\sigma_{n}=100 \mathrm{MPa}$

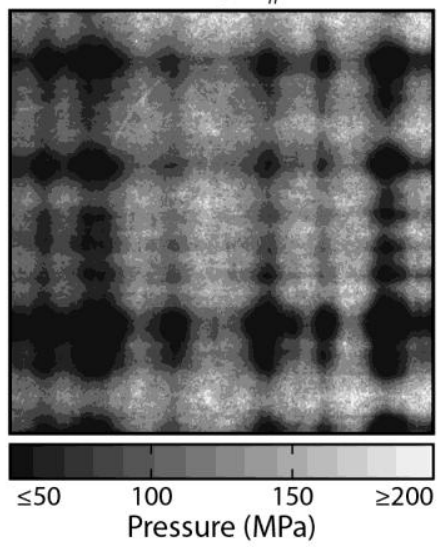

(e) Sim. pressure, $\sigma_{n}=100 \mathrm{MPa}$

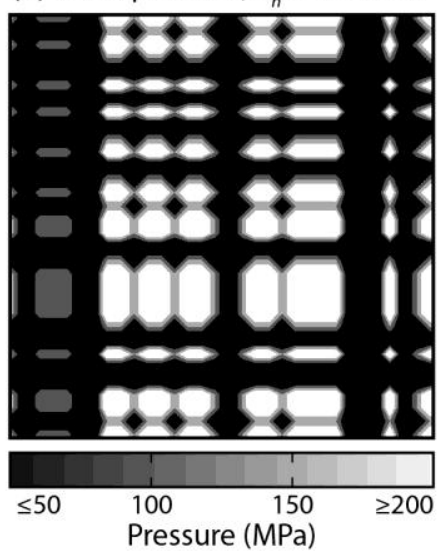

(c) Pressure map, $\sigma_{n}=300 \mathrm{MPa}$

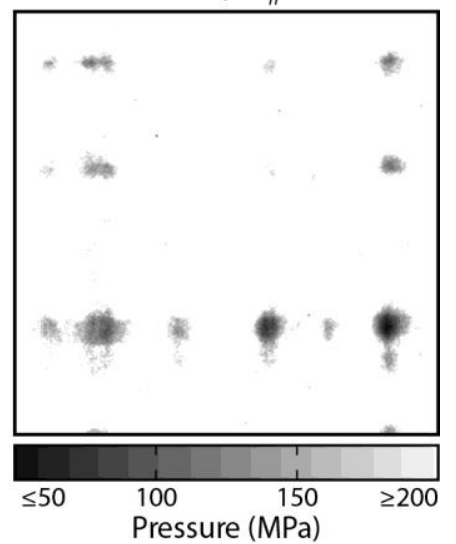

(f) Sim. pressure, $\sigma_{n}=300 \mathrm{MPa}$

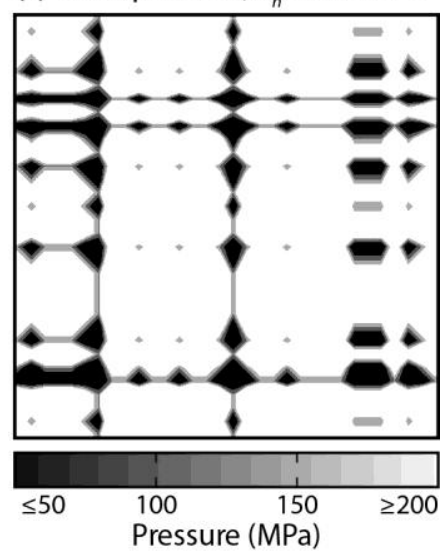

Figure 5.13. (a) Backlit photograph of a pristine $\mathrm{L}=30 \mathrm{~mm}$, thin $\left[0^{\circ} / 90^{\circ}\right]_{8} \mathrm{HB} 26$ laminate test sample. Pressure sensitive film read after compressive loading to a nominal stress of $(b) \sigma_{n}=100$ $\mathrm{MPa}$ and (c) $\sigma_{n}=300 \mathrm{MPa}$. Simulations of the backlit image (c) and pressure maps (e and f) are shown along the bottom row.

The pressure is highly non-uniform, with large areas exposed to $<50 \mathrm{MPa}$ of pressure and other small areas to $>200 \mathrm{MPa}$. The low pressure bands are aligned with the $\mathrm{X}$ - and $\mathrm{Y}$ - fiber directions, and lower pressure regions existed at intersections of the low pressure bands. The persistence of these low pressure regions was tested by reloading the sample, with a new pressure film, to $\sigma_{n}=300 \mathrm{MPa}$, Figure 5.13(c). While the majority of the area surpassed the $200 \mathrm{MPa}$ saturation limit of the film, portions of the low-pressure nodes were still present, with some registering a local pressure $<50 \mathrm{MPa}$. The location of the low pressure regions were well correlated with the dark regions in the optical 
transmission image of the sample taken before testing, Figure 5.13(a). This indicates that the high optical (and ultrasonic attenuation) regions, which were shown by $\mu \mathrm{XCT}$ to contain high void concentrations due to missing fibers, are locations of significantly reduced load carrying capacity.

The pressure distribution of a thick, 240 ply, HB26 laminate has also been investigated. The optical image of the sample's central region again indicated the presence of fiber aligned high attenuation bands, Figure 5.14(a). These were well correlated with low pressure regions recorded by the pressure film after loading the sample to $\sigma_{n}=100 \mathrm{MPa}$, Figure 5.14(b). However, the pressure distribution was more uniform (more centered around $100 \mathrm{MPa}$ ) than the thinner laminate. Subsequent loading of the sample to $\sigma_{n}=300 \mathrm{MPa}$ completely saturated the pressure film, indicating that no area registered a pressure below $200 \mathrm{MPa}$. It therefore appears that increasing the laminate thickness, improves the uniformity of load support. 
(a) Backlit photograph

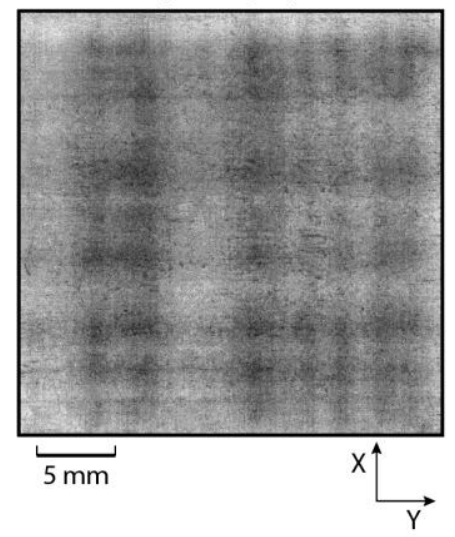

(d) Sim. backlit image

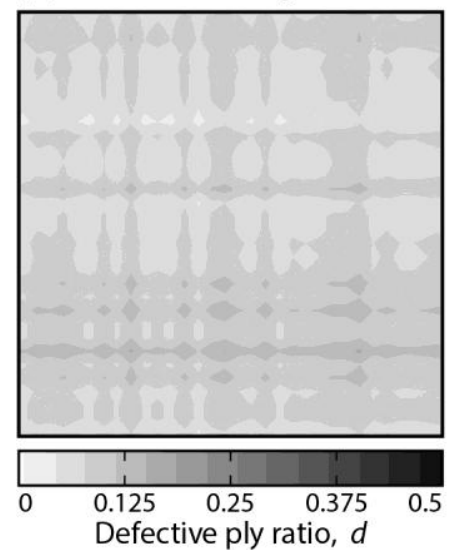

(b) Pressure map, $\sigma_{n}=100 \mathrm{MPa}$

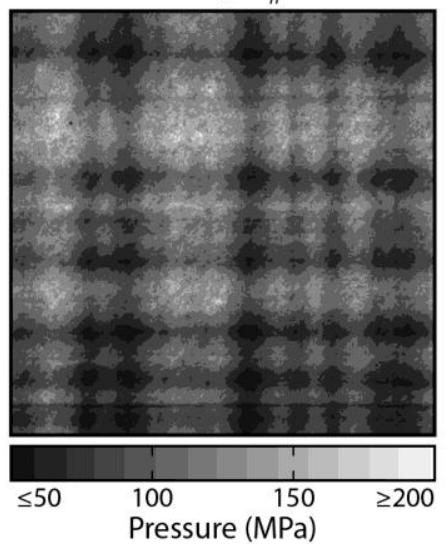

(e) Sim. pressure, $\sigma_{n}=100 \mathrm{MPa}$

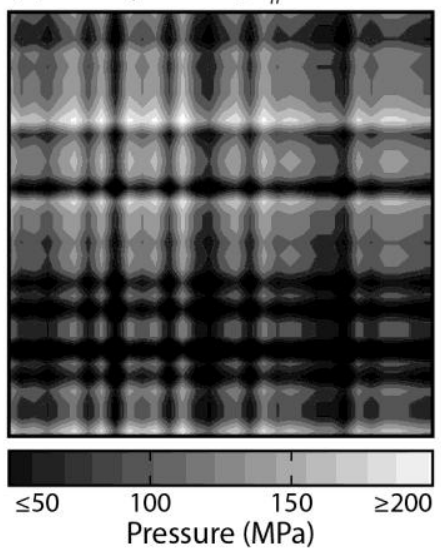

(c) Pressure map, $\sigma_{n}=300 \mathrm{MPa}$

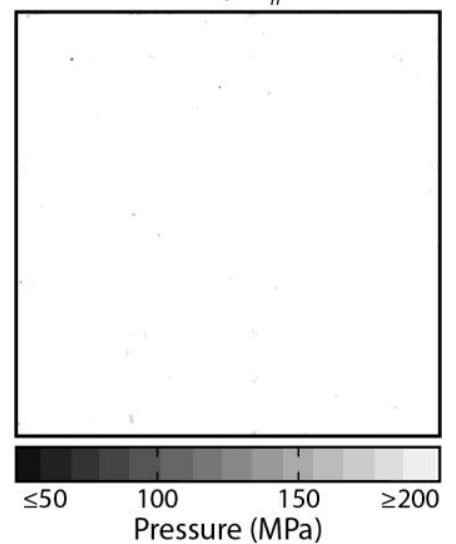

(f) Sim. pressure, $\sigma_{n}=300 \mathrm{MPa}$

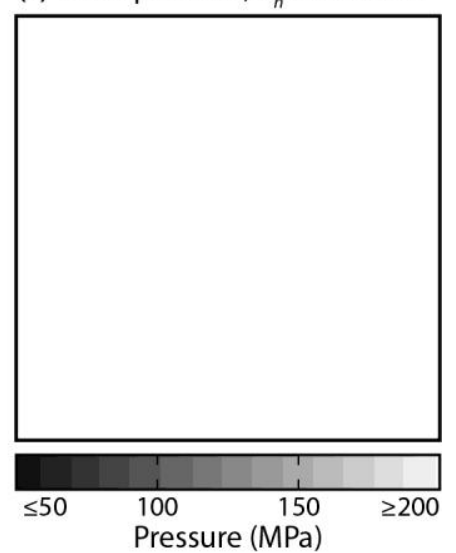

Figure 5.14. (a) Backlit photograph of a pristine $L=30 \mathrm{~mm}$, thick $\left[0^{\circ} / 90^{\circ}\right]_{120} \mathrm{HB} 26$ laminate test sample. Pressure sensitive film readings from uniform compressive loading to a nominal stress of (b) $\sigma_{n}=100 \mathrm{MPa}$ and (c) $\sigma_{n}=300 \mathrm{MPa}$. Simulations of the backlit image (c) and pressure maps $(e$ and $f$ ) are shown along the bottom row.

Figure 5.15(a) shows a $\mu \mathrm{XCT}$ cross-sectional view of an HB26 sample showing the numerous tunnel cracks within a 16 ply laminate. After scanning, the sample was loaded to $\sigma_{n}=400 \mathrm{MPa}$ which is above the transverse yield strength of the plies, unloaded, and rescanned. A cross-section through the same location of the sample, after loading, is shown in Figure 5.15(b). The compressive stress was sufficiently high to cause transverse plastic (Poisson) deformation of the plies, and closure of the tunnel cracks. This was observed to have occurred with no permanent change in sample thickness. 
(a) Prestine material

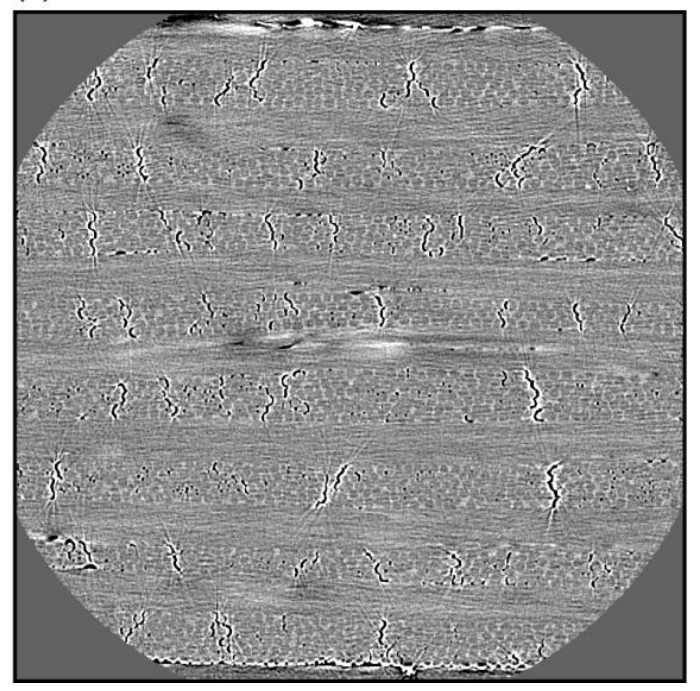

(b) Post $400 \mathrm{MPa}$ load-unload

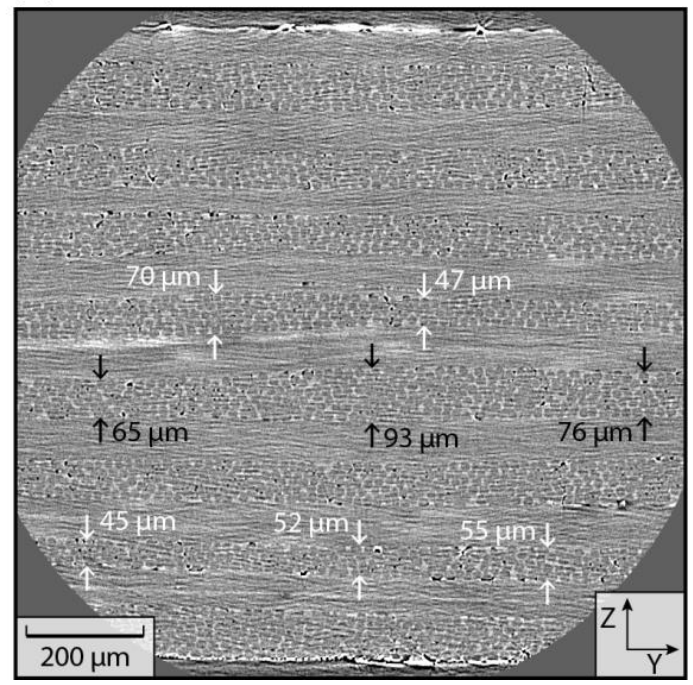

Figure 5.15. Two $\mu X C T$ cross-sections of a single location within a $\left[0^{\circ} / 90^{\circ}\right]_{8} H B 26$ sample: (a) In its pristine state and (b) after compressing to $400 \mathrm{MPa}$ and unloading. Measurements reveal variability in both inter- and intra-ply thickness.

To determine if the presence of the tunnel cracks had any effect upon the laminate compressive strength, five $L=35 \mathrm{~mm}, 16$ ply, HB26 samples were first loaded to $\sigma_{n}=$ $400 \mathrm{MPa}$ and unloaded. A $5 \mathrm{~mm}$ border around the edge of each sample, which included the shear lag region, was then removed. The now $L=25 \mathrm{~mm}$ samples were then reloaded to failure and their average maximum strength found to be $1.01 \pm 0.05 \mathrm{GPa}$. Nonpreloaded samples had an average maximum strength of $1.05 \pm 0.09 \mathrm{GPa}$. We conclude that tunnel cracks have negligible effect on compressive strength.

Detailed examination of $\mu \mathrm{XCT}$ scans, Figure 5.15, also indicated the presence of a large variance in ply thickness. The thicknesses of three $0^{\circ}$ plies at various locations along the Y-axis are marked on Figure 5.15(b). Each ply was found to vary in thickness by at least $20 \mu \mathrm{m}$, and the average thickness of the ply marked by black arrows seems larger than the plies identified by white distance markers. A varying ply thickness is consistent with an uneven distribution of fibers during tape fabrication as exposed by 
$\mu \mathrm{XCT}$, Figure 5.12. The recently developed transverse compressive strength model of Atwood et al. [2] predicts a significant effect of ply thickness (see Eqs. A4 and A6).

To experimentally investigate the role of ply thickness variability we fabricated samples from single $0^{\circ}$ ply HB26 pre-preg tape with a bimodal ply thickness of either $\left[0^{\circ} 2 / 90^{\circ}\right]_{5}$ or $\left[0^{\circ}{ }_{3} / 90^{\circ}\right]_{4}$, and compared their strength to experiments and predictions for equivalent uni-modal $\left[0_{2}^{\circ} / 90^{\circ}{ }_{2}\right]_{4}$ and $\left[0_{3}^{\circ} / 90^{\circ}\right]_{3}$ laminates. Laminate consolidation followed the same procedure outlined in Section 2.3, except the pre-preg tape stack was heated and pressed in a pocket die to prevent lateral extrusion and concomitant ply thinning. The compressive strength results and predictions (using Eq. ( 5-6)), are plotted in Figure 5.16. Experimental results for samples made from the $0^{\circ}$ ply pre-preg tape indicate that as the monoply thickness was increased from $\left[0^{\circ} / 90^{\circ}\right]_{8}$ to $\left[0_{3}^{\circ} / 90^{\circ}\right]_{3}$, the transverse compressive strength at fixed sample size $L$ decreased (as a result of increased shear lag lengths). The experimental results also agreed well with predictions using the $\left[0^{\circ} / 90^{\circ}\right]_{2} \mathrm{HB} 26$ tape material properties. Furthermore, the compressive strength of the $\left[0^{\circ}{ }_{2} / 90^{\circ}\right]_{5}$ or $\left[0^{\circ}{ }_{3} / 90^{\circ}\right]_{4}$ bimodal laminates was similar to that of the $\left[0^{\circ}{ }_{2} / 90^{\circ}{ }_{2}\right]_{4}$ and $\left[0^{\circ}{ }_{3} / 90^{\circ}\right]_{3}$ laminates, respectively. Thus, the compressive strength of a bimodal laminate is controlled by the response of its thickest ply. 


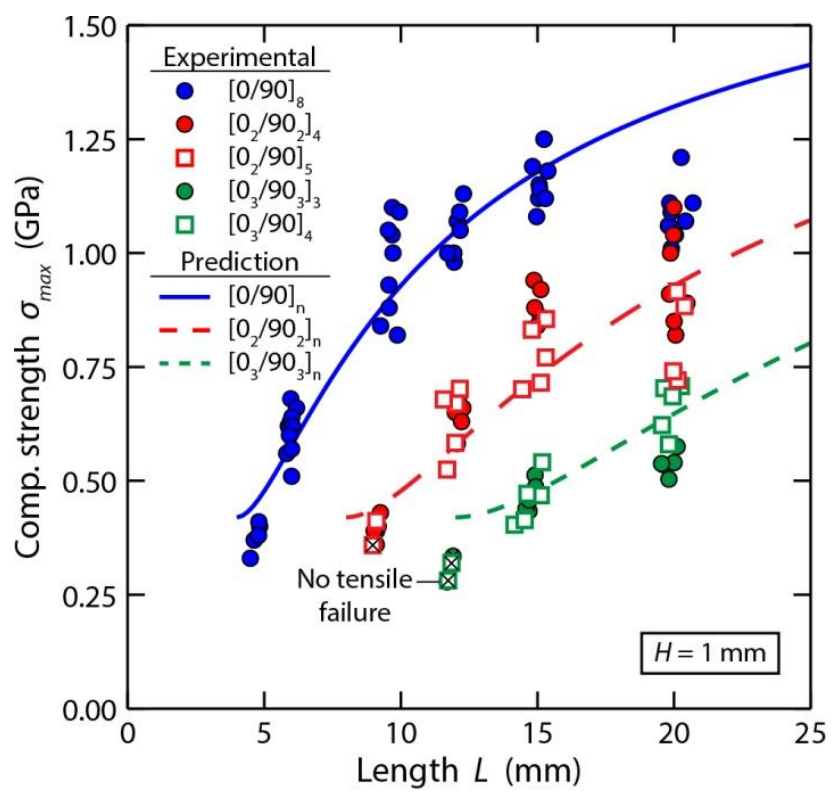

Figure 5.16. The compressive strength, $\sigma_{\max }$, as a function of sample length, $L$, of laminates of varying ply thickness, $h$, made from a unidirectional HB26 pre-preg ply.

\subsection{Modeling and simulation}

The effect of missing fiber (void-like) defects upon the transverse compressive strength can be understood by analysis of a simple model based on stress-shielding of defected regions. The following assumptions were used to assemble the model:

(i) Only defects due to missing fibers are analyzed.

(ii) Defects in a ply were collinear with the fiber direction.

(iii) Defects were of infinite length.

(iv) The defect width was a multiple of the fiber diameter.

(v) The defect to ply thickness fraction, $\eta$, was defined as the ratio of the thickness of a single defect, $h_{d}$, to the ply thickness $h$. Thus, $0 \leq \eta=h_{d} / h \leq$ 1. 
(vi) The defect locations were random and independent, occurring with a probability $p$.

The model has been used to investigate the HB26 grade; the material upon which the most comprehensive defect characterization could be performed.

The X-Y plane of a unidirectional ply of square in-plane dimension $L$ was discretized into strips of uniform width, Figure 5.17(a). Each strip had an equal probability $p$ of being a missing fiber defect. If $2 \mathrm{n}=N$ plies were generated in this manner, and combined to form a $\left[0^{\circ} / 90^{\circ}\right]_{\mathrm{n}}$ laminate of thickness, $H$, a square grid with cross-hatches of overlapping (elongated void-like) defects is generated. The sum of the void thicknesses, $h_{d}$, assuming $\eta=1$, in the Z-direction can then be computed as a function of $(\mathrm{X}, \mathrm{Y})$ location and is denoted $r=r(\mathrm{X}, \mathrm{Y})$. This quantity ranges in discrete values from 0 to $N$, Figure 5.17(a) and (c). The defective ply to laminated ply ratio is given by $d(\mathrm{X}, \mathrm{Y})=r(\mathrm{X}, \mathrm{Y}) / N$. The model was implemented in MATLAB (software version R2013a) by assuming randomly distributed defect locations in each ply. For each strip within a ply, the function "rand" generated a pseudo-random number between 0 and 1. The numbers were then compared with the pre-established probability of a defect, $p$, and values less than $p$ were designated a void. The $\left[0^{\circ} / 90^{\circ}\right]_{\mathrm{n}}$ laminate was then assembled, and the values of $r$, and then $d$, calculated. 
(a) Simulated $\left[0^{\circ} / 90^{\circ}\right]_{4}$ lay-up with defects

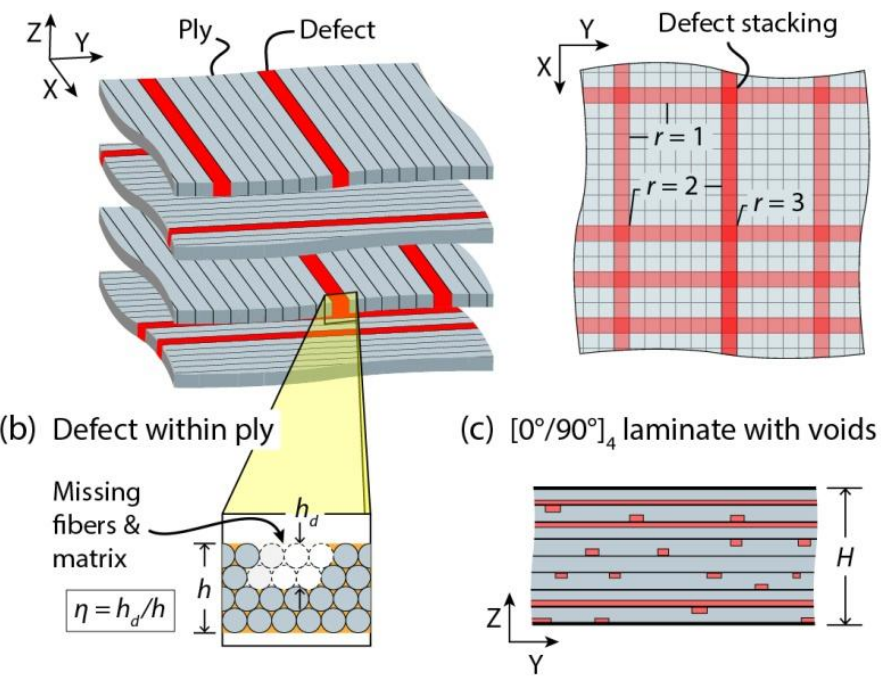

(d) Stress shielding model

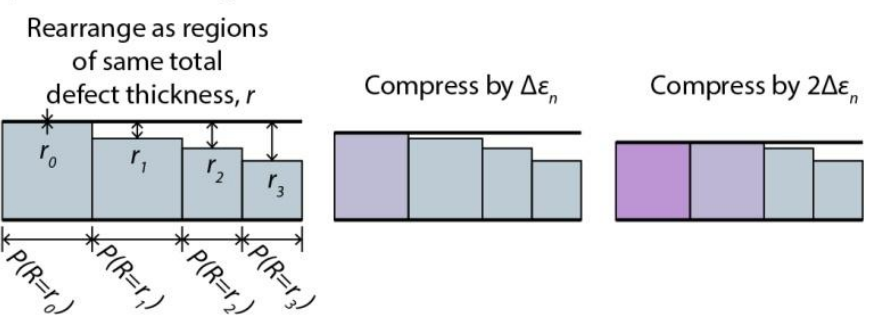

Figure 5.17. (a) Schematic illustration showing how sets of missing fibers within a ply lead to a laminate with fiber aligned void-like defects. (b) Definition of the defect ply fraction, $\eta$. (c) A $\left[0^{\circ} / 90^{\circ}\right]_{4}$ laminate with defect voids. (d) Hypothetical rearrangement of voids where the thickness of overlapping voids is summed and the laminate area is then divided into areas factions, $P$, having the same overlapping void thickness. Compression of the laminate to a nominal strain, $\varepsilon_{n}$, will then lead to incremental loading of laminate area.

Figure 5.18(a) shows a simulation of a 16 ply square laminate with a plate thickness to void width ratio of 150 and a void probability $p=0.05$. The contrast represents the value of $d$ : white regions being defect free and black regions having a defect in at least half of the overlapping plies. The majority of the area is observed either to be defect free or to have one defect. Slightly darker grey regions are seen at the crossing of orthogonally oriented defects or as bands where two collinear defects overlap. The area fraction of overlapping defects is however small. The effects of increasing the probability of missing fiber defects to 0.1 and then 0.15 is shown in Figure 5.18(b) and 
(c). A comparison with the backlit image of a 16 ply HB26, Figure 5.8(a), indicates a best match for a defect probability $p=0.1$.

(a) $\left[0^{\circ} / 90^{\circ}\right]_{8^{\prime}} p=0.05$

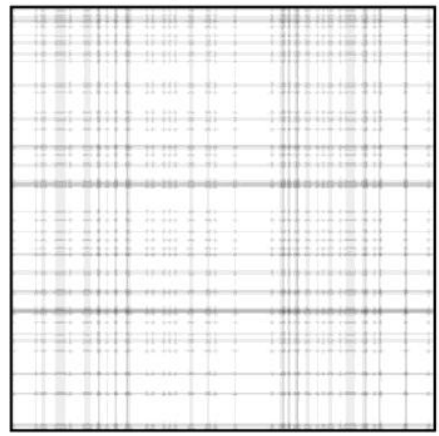

(b) $\left[0^{\circ} / 90^{\circ}\right]_{8^{\prime}} p=0.10$

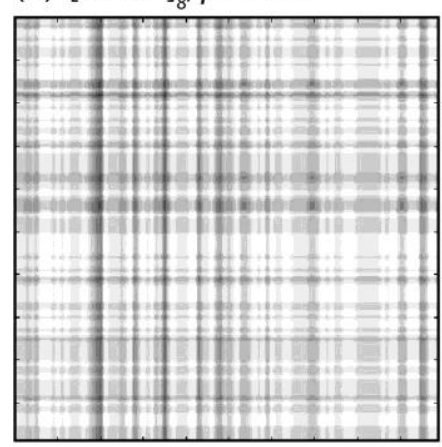

(c) $\left[0^{\circ} / 90^{\circ}\right]_{8^{\prime}} p=0.15$

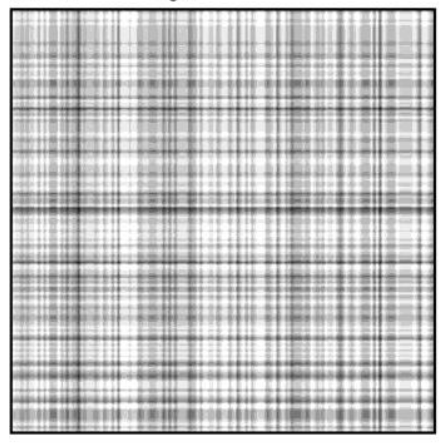

(d) $\left[0^{\circ} / 90^{\circ}\right]_{30^{\prime}} p=0.10$

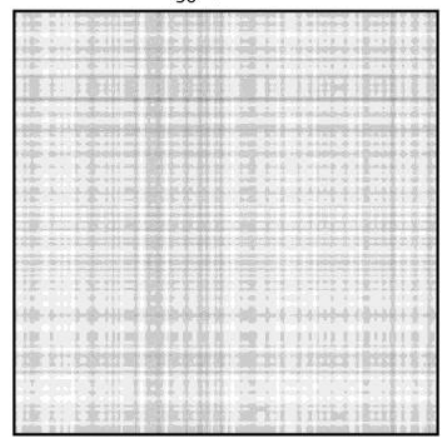

(e) $\left[0^{\circ} / 90^{\circ}\right]_{60^{\prime}} p=0.10$

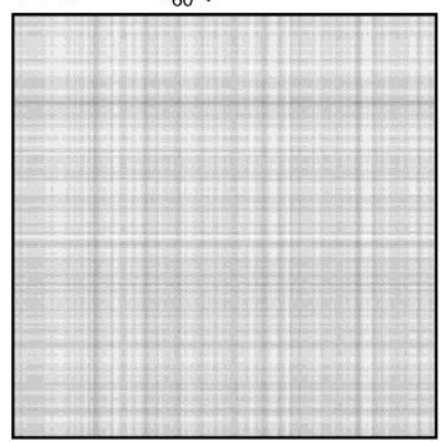

(f) $\left[0^{\circ} / 90^{\circ}\right]_{120^{\prime}} p=0.10$

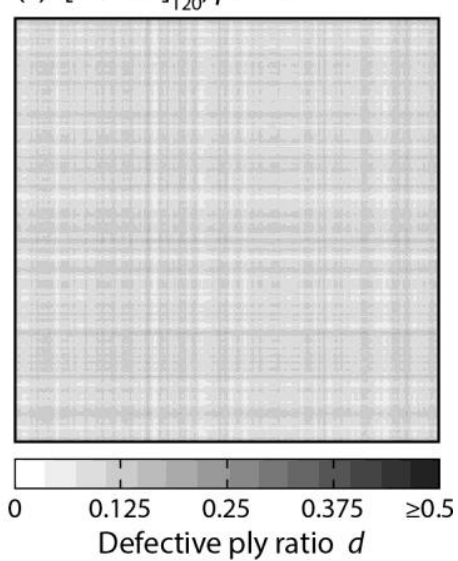

Figure 5.18. Simulations of a $\left[0^{\circ} / 90^{\circ}\right]_{8}$ laminate with defects assuming a plate width to void width ratio of 150 and a void probability of $(a) p=0.05$, (b) $p=0.10$ and (c) $p=0.15$. Additional simulations using $p=0.10$ for lay-ups of increasing thickness are shown in $(d)$ $\left[0^{\circ} / 90^{\circ}\right]_{30}$, (e) $\left[0^{\circ} / 90^{\circ}\right]_{60}$ and $(f)\left[0^{\circ} / 90^{\circ}\right]_{120}$.

Figure 5.19(a) shows the probability mass distributions of defect overlap in the Zdirection as a function of the defective ply ratio, $d$, for these same values of defect 
probability, $p$. The probability mass, $P$, is an estimate of the area fraction of an infinitely long and wide laminate having a given value of $d$. We note it is well approximated by a binomial distribution:

$$
P(R=r=N d)=\left(\begin{array}{c}
N \\
N d
\end{array}\right) p^{N d}(1-p)^{N(1-d)}
$$

where $R$ is a discrete random variable describing the number of overlapping defects and ranging in value from 0 to $N, P$ is the relative likelihood that the random variable $R$ has a value of $r$ (again $r=N d$ ) overlapping defects, and $\left(\begin{array}{c}N \\ N d\end{array}\right)$ is the number of possible combinations that results in $r$ overlapping defects in a laminate $N$ plies thick. For a defect probability of $p=0.05$, the probability of there being no void-like defects $(d=$ 0 ), which happens to be the most probable outcome, was $P=0.44$. This is the equivalent to $44 \%$ of the area being defect free through a laminate's thickness. The value of $P$ then monotonically decreased with each increment in $d$. Increasing the void probability $p$ changed the shape of the distribution. The probability density $P$ for $d=0$ (no defects) decreased towards zero, the probability density peaked at larger values of $d$, and the variance in $d$ increased. These behaviors were evident in the simulated laminates, Figure $5.18(\mathrm{a}-\mathrm{c})$ 
(a) Defect probability dependence

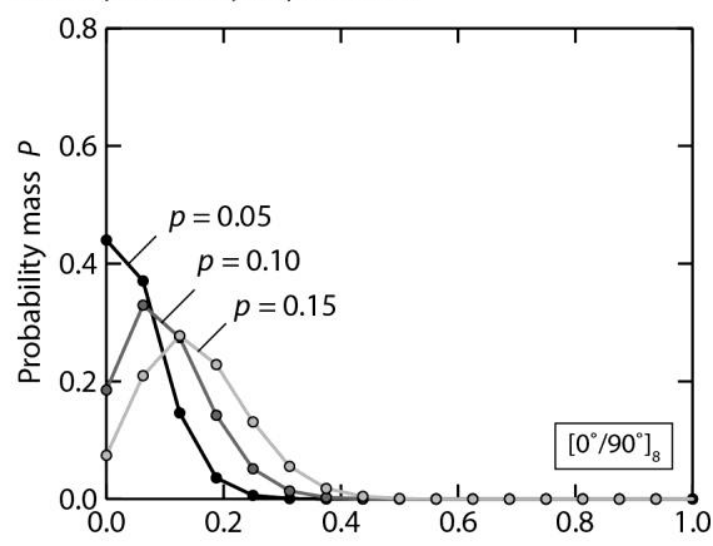

(b) Thickness dependence

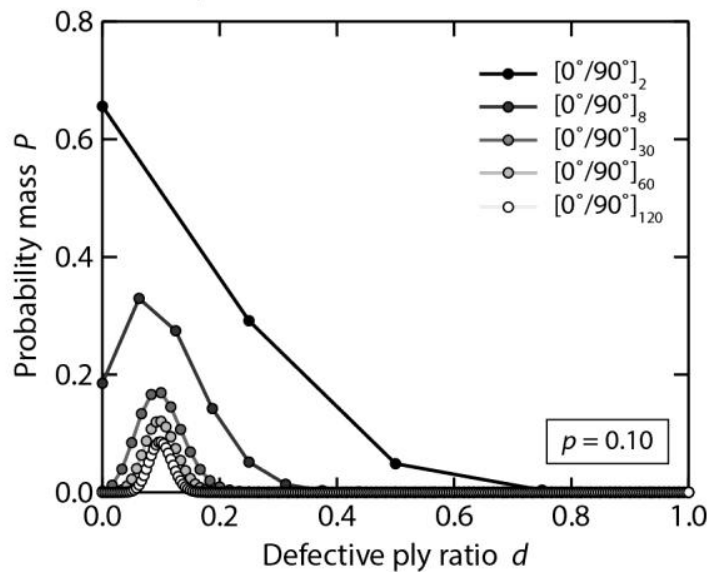

Figure 5.19. Probability mass $P$ of the local fraction of defective plies $d$. Results are shown in (a) for a 16 ply laminate with various defect probabilities $p$ and in (b) for the number of plies in a laminate assuming $p=0.10$.

The effect of increasing the laminate thickness for a fixed defect probability $p=$ 0.10, can be seen in the simulated images Figure 5.18(d-f). Increasing the number of plies reduced the band contrast, especially at band overlaps, and homogenized the image.

These observations are consistent with backlit images of 16 and 120 ply laminates, Figure 5.7 and Figure 5.8 respectively, and agree with a narrowing of the predicted probability mass towards a value of $d=p=0.1$ as the laminate thickness increased from $\left[0^{\circ} / 90^{\circ}\right]_{8}$ to $\left[0^{\circ} / 90^{\circ}\right]_{120}$, Figure 5.19(b). 
The loading behavior of one of these infinite area $(L \rightarrow \infty)$ laminates, having a random distribution of missing fiber (elongated void) defects like the one depicted in Figure 5.17(a-c) could then be modeled using the defect mass $P(d)$ given by Eq. ( 5-7). A laminate could hypothetically be rearranged into area fractions $P(d)$ of defective ratios $d$, as shown at the left of Figure 5.17(d). If the laminate were then subject to an initial compressive displacement, the force would be first picked-up by the defect free area, followed by the area having only one defect in thickness after further displacement increments, and so on, Figure 5.17(d). If a region in such a sample fails when the compressive strength of an infinite laminate, $\sigma_{i}^{\infty}$, given by Eq. ( 5-4), is reached, the load supported by that region can be reassigned to other, yet to fail regions. Eventually, this redistribution of load will exceed the strength of the surviving laminate, and will define the laminates transverse compressive strength.

To implement such a failure model, it is assumed that neighboring regions are independent and do not laterally transfer loads. It also assumes no shear lag region exists at the sides of each region. Such a model was implemented with MATLAB. For an $N$ ply laminate, an index $i$ was used to track each of the $N+1$ regions with a specific value of $d$, each having an area fraction $P(d)$. An iterative loop was used to increment the nominal compressive strain applied to the laminate by $\Delta \varepsilon_{n}$, and then calculate the regional strains and stresses, and check for region failure. Starting at zero strain, the nominal strain applied to the laminate was:

$$
\varepsilon_{n}(j)=\varepsilon_{n}(j-1)+\Delta \varepsilon_{n}
$$

where $j$ is an index accounting for both the number of increments in strain and the number of failed regions. The region specific nominal strain $\bar{\varepsilon}$ was then calculated by: 


$$
\bar{\varepsilon}(i, j)=\left\{\begin{aligned}
0, & \eta d(i)<0 \\
\frac{\varepsilon_{n}(j)-\eta d(i)}{1-\eta d(i)}, & \text { otherwise }
\end{aligned}\right.
$$

where $\eta$ has been previously defined as the defect ply fraction, Figure 5.17(b).

The region specific nominal stress $\bar{\sigma}$ was then calculated by rearrangement of Eq. (5-2), using the following inputs:

$$
\bar{\sigma}(i, j)=-\sigma_{33}\left(\varepsilon_{33}=-\bar{\varepsilon}(i, j)\right)
$$

The called upon equation provides the compressive stress within an infinite laminate which is justified given our assumptions of no shear lag length or interaction between regions. The nominal laminate stress $\sigma_{n}^{\infty}$ was then calculated by:

$$
\sigma_{n}^{\infty}(j)=\sum_{i=1}^{N+1} \bar{\sigma}(i, j) P(i) m(i, j-1)
$$

Where $m$ was an index tracking the failure of a region using:

$$
m(i, j)= \begin{cases}1, & \bar{\sigma}(i, j)<\sigma_{i}^{\infty} \\ 0, & \bar{\sigma}(i, j) \geq \sigma_{i}^{\infty}\end{cases}
$$

In the event of a local region failure, i.e. $m(i, j)<m(i, j-1)$, then $j$ was incremented by 1 , the laminate nominal strain was updated to be $\varepsilon_{n}(j)=\varepsilon_{n}(j-1)$, and $\sigma_{n}^{\infty}(j)$ was calculated using $m(i, j)$. The incremental straining of the laminate, restarting from Eq. ( 5-8), continued until either:

- $\quad$ All of the regions failed, i.e. $\sum_{i=1}^{N+1} m(i, j)=N+1$,

- $\quad$ Or the stress fell to half of peak strength, i.e. $\sigma_{n}^{\infty}(j)<\frac{1}{2} \sigma_{\max }^{\infty}$, where $\sigma_{\max }^{\infty}$ is the maximum value of $\sigma_{n}^{\infty}(j)$ 
The HB26 properties provided in Table 2.2 were used as inputs for the model. The stress-strain relation in Eq. ( 5-2) required additional values for the transverse ply modulus, longitudinal ply modulus, shear modulus and transverse Poisson's ratio. Values from Attwood et al. [2] for $E_{m}=15 \mathrm{GPa}, E_{f}=70 \mathrm{GPa}$, and $G_{12}=3 \mathrm{MPa}$, were used. The transverse Poisson's ratio was measured in Section 3.5, $v=0.5$. The strain increment was set to $\Delta \varepsilon_{n}=0.0001$, equivalent to $\sim 1 / 4,000$ of the experimentally observed failure strain.

The predicted nominal stress normalized by $\sigma_{i}^{\infty}$ verses nominal strain response for a defect free $(p=0)$ laminate is shown by the dashed line in Figure 5.20. The stress linearly increased with strain, and the laminate catastrophically failed at $\sigma_{\max }^{\infty} / \sigma_{i}^{\infty}=1$ and $\varepsilon_{n}=0.15$. The load-shielding model was then run for a 16 ply HB26 laminate using a defect probability of $p=0.10$ and a defect ply thickness fraction $\eta=0.4$. The value of $\eta$ was motivated by XCT imaging, Figure 5.10, and provided an effective void fraction of $p \eta=4$ vol. $\%$. In contrast with the homogenous laminate, the initial stress-strain response was more compliant, with laminate stiffness increasing to a strain of $\varepsilon_{n} \approx 0.08$. The rate of stiffening decreased with each newly engaged region of $d$, since the area fraction of each newly engaged region was an increasingly smaller fraction of the total engaged area, as apparent in Figure 5.19(b). After this stiffening stage, the stress linearly increased up to a strain of $\varepsilon_{n}=0.15$, where the void free region, which accounted for $19 \%$ of the laminate area, again failed, and the stress abruptly dropped. The laminate continued to support a reduced load, and partially failed twice more before the stress fell below $1 / 2 \sigma_{\max }^{\infty}$, which in this scenario equaled $\sigma_{\max }^{\infty} / \sigma_{i}^{\infty}=0.75$. Comparing the predicted response to experimental results of 16 ply, $L=12 \mathrm{~mm}$ HB26 samples, Figure 5.5(a), a similar initial stiffening behavior that transitioned into a linear elastic response was observed (Atwood 
et al. [2] also observed this initial stiffening behavior). Only a single experimental drop in load was observed for samples 1 and 2, but sample 3 failed in multi-steps, during which the load similarly fell and subsequently recovered as in the predictions.

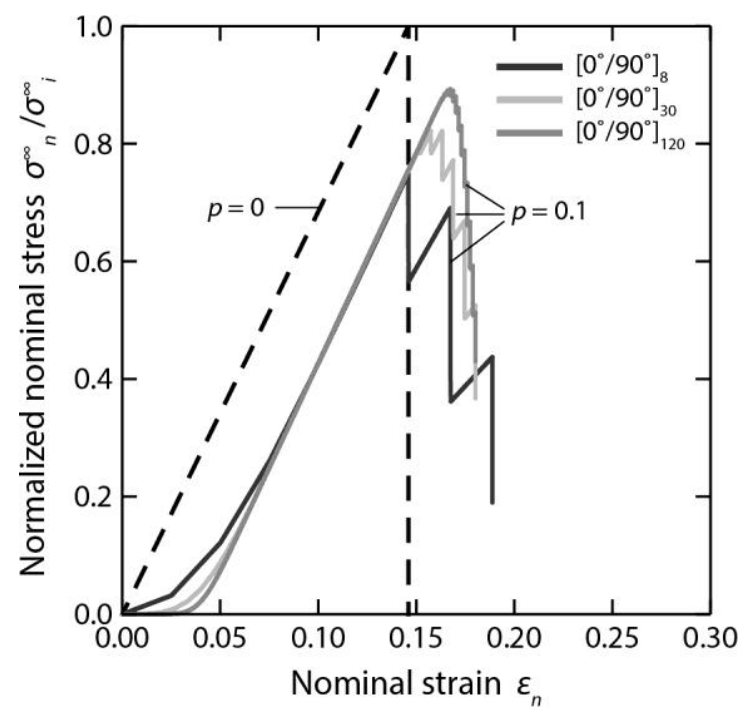

Figure 5.20. Predicted nominal stress, $\sigma_{n}$, normalized by the ideal plateau strength, $\sigma^{\infty}{ }_{i}$, and plotted as a function of strain, $\varepsilon_{n}$, for HB26 laminates of infinite in-plane dimension $(L \rightarrow \infty)$, given $\eta=0.4$.

When the laminate thickness was increased to 80 and then 240 plies, the initial simulated stress was very low until the strain $\varepsilon_{n} \approx 0.02$, Figure 5.20. However with further loading, the response stiffened at a greater rate than that of a 16 ply laminate before stabilizing with the similar linear-elastic response. Recall from Figure 5.19 that an 80 ply laminate has a probability density of a defect free area of approximately zero, and the variance in $d$ is much lower than that for a 16 ply laminate. These two defect characteristics were responsible for the delay in hardening, and then the more rapid stiffening rate, of the thicker laminates. Failure of the first region within the 80 ply laminate again occurred at a strain of $\varepsilon_{n}=0.15$, but since it occurred in a domain of very small area, the load continued to increase at a similar rate. By reducing the variance in $d$, 
the load was more evenly distributed over a larger fraction of the area and allowed the laminate to reach a strength $\sigma_{\max }^{\infty} / \sigma_{i}^{\infty}=0.84$. This failure strength was further increased as the number of plies was increased to 240 , consistent with a further decrease in the variance of $d$.

The normalized nominal compressive strength has been plotted as a function of the number of plies for the same values of $\eta=0.4$ and $p=0.10$ in Figure 5.21(a). The predicted strength for the thinnest laminate started at $\sigma_{\max }^{\infty} / \sigma_{i}^{\infty}=0.75$ and initially decreased with increasing number of plies because of the decreasing void free area fraction and the large variance in $d$. Once the laminate became more homogenous, the strength began to rise with ply number, and then approached an asymptotic strength that decreased with $p$. For a 300 ply $(H \approx 20 \mathrm{~mm}$ for HB26) laminate, with $p=0.1$, the strength reached $\sigma_{\max }^{\infty} / \sigma_{i}^{\infty}=0.90$. The experimental data from Figure 5.4(a) is also plotted in Figure 5.21(a) for comparison. Shown is the average (grey circles) and standard deviation for the normalized strength values for the data ranging from $L=20$ to $30 \mathrm{~mm}$. A similar waning increase with laminate thickening is observed. 
(a)

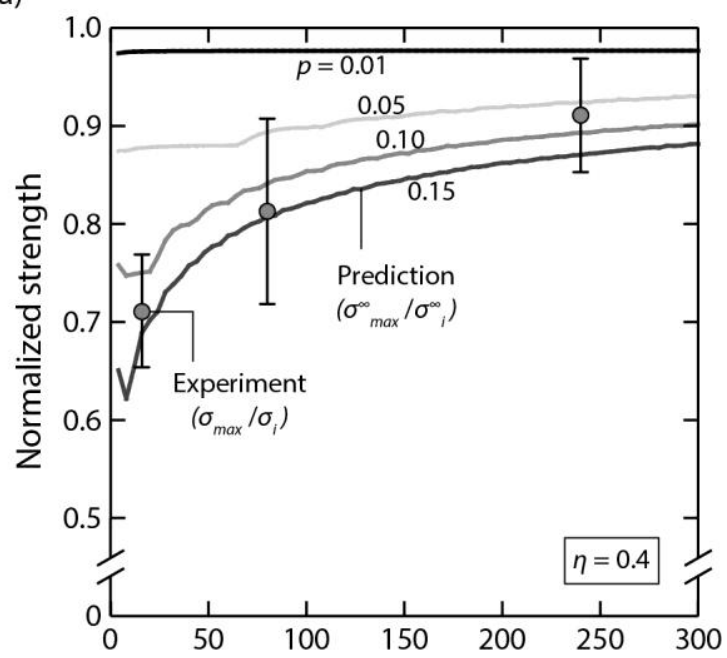

(b)

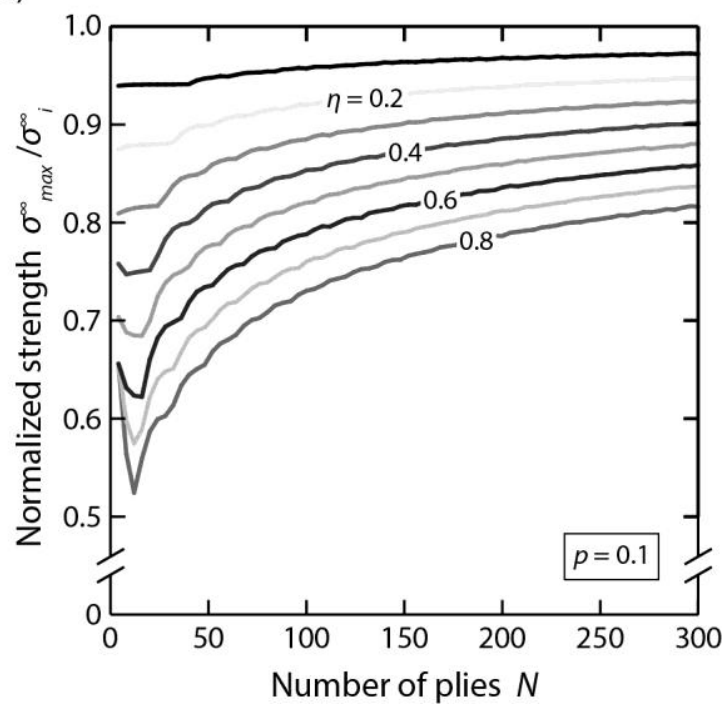

Figure 5.21. Predicted strength as a function of the number of laminated plies, $N$, for an HB26 laminate of infinite in-plane dimension $(L \rightarrow \infty)$. (a) Assumes $\eta=0.4$ and investigates the influence of defect probability, $p$, while ( $b$ ) shows the effect of defect to ply thickness fraction, $\eta$, for $p=0.10$. Also plotted as a function of the number of laminated plies in (a) is the normalized average strength and standard deviation for HB26 samples for $L=20 \mathrm{~mm}$ to $30 \mathrm{~mm}$.

The effect of the defect ply fraction $\eta$ upon the normalized strength verses laminate thickness relation is shown in Figure 5.21(b) for a situation where $p=0.1$. Increasing $\eta$ significantly lowered the strength; most noticeably in thin laminates where it reached only $50 \%$ the strength of a defect free laminate. Increasing the void probability $p$, 
decreasing the number of plies $N$ and increasing the defect ply faction $\eta$ all reduce the laminate compressive strength.

For a final comparison to experiments, the model was utilized to replicate the pressure distribution study. Using the same values of $p=0.10$ and $\eta=0.4$, a 16 ply laminate was simulated, Figure 5.13(d), to match the backlit image in Figure 5.13(a). When compressed to $\sigma_{n}=100 \mathrm{MPa}$, a similar pressure distribution to the experiments, with load shielding of regions containing a higher defect concentration, was seen, Figure 5.13(b) and (e). After incrementing the stress to $\sigma_{n}=300 \mathrm{MPa}$, pockets of low pressure still existed at the intersection of void bands, similar to the experimental behavior, Figure 5.13(c) and (f). The simulated 240 ply laminate, Figure 5.14, also compared well against the experimental results, revealing a more homogenized pressure distribution in comparison to the 16 ply laminate.

\subsection{Concluding remarks}

We have experimentally observed that increasing the thickness and area of $\left[0^{\circ} / 90^{\circ}\right]$ cross ply UHMWPE fiber reinforced plastic composite laminates leads to an increase in the through-thickness (transverse) compressive strength of the laminate. In some material systems, the compressive strength of the thickest laminates approaches that predicted by a recently proposed indirect tension model [2], while the thinnest laminates have compressive strengths that are $30 \%$ to $40 \%$ below these predictions.

Using a combination of optical, ultrasonic and $\mu \mathrm{XCT}$ imaging methods, we have discovered that UHMWPE fiber-reinforced laminates contain elongated void-like defects 
consisting of pockets of missing fibers and resin that span long distances $(>150 \mathrm{~mm})$ in the fiber direction of a ply. Tunnel cracks arising from anisotropic thermal contraction during the cooling stage of the consolidation process are also observed. However, the removal of tunnel cracks, by cold pressing the laminate, had no effect upon the transverse compressive strength of the laminate.

By placing a high spatial resolution pressure sensitive film between a laminate sample and the compression platens, we have discovered that the pressure distribution over the area of the laminate is highly non-uniform. The films show low pressure bands that are aligned with the directions of the fibers, and correspond to the locations of "missing fiber" defect scattering regions as observed with optical and ultrasonic imaging. Using $\mu \mathrm{XCT}$, we find that the missing fiber defects can be traced to the as-received prepreg tape. An uneven distribution of the small diameter $(\sim 15 \mu \mathrm{m})$ fibers results in a tape with considerable variability in ply thickness and fiber- and resin-free voids within a ply. A $20 \mathrm{MPa}$ hot-pressing procedure used to consolidate the laminates fills some of these defects by lateral flow of material. The progressive removal of these defects may explain the decrease in ultrasonic attenuation [6] and the higher ballistic performance reported in Refs. $[12,13]$ when the consolidation pressure was increased.

Ply variability also affected the transverse compressive strength, which was made apparent by comparison of monoply thickness laminates, $\left[0_{2}^{\circ} / 90^{\circ}{ }_{2}\right]_{4}$ and $\left[0^{\circ}{ }_{3} / 90^{\circ}{ }_{3}\right]_{3}$, to their bimodal ply thickness variants, $\left[0^{\circ} 2 / 90^{\circ}\right]_{5}$ and $\left[0_{3}^{\circ} / 90^{\circ}\right]_{4}$. The compressive strength of the bimodal ply thickness laminates decreases with increasing $0^{\circ}$ ply thickness and matched the values for a monoply laminate made from the thickest ply. A laminate having variability in ply thickness may then have regions prone to earlier failure, and 
may explain the observed variability in compressive strength, where the lowest strength laminates produced the highest acoustic activity. These observations may warrant future study of ply variability.

A simple statistical model has been assembled to investigate the effect of missing fiber defects upon the compressive strength of a laminate. The model assumed defects were randomly located and successfully simulated optical images of bands of missing fiber defects stacked in the thickness direction of laminates. By accounting for load shielding of the voided regions, substantial reductions in transverse compressive strength were predicted when the missing fiber defect probability was in the $10 \%$ range (corresponding to a total void fraction of 4 vol.\%). The model also predicted an increase in compressive strength with sample thickness; consistent with experiments.

These findings may be of particular interest to the ballistic impact community since a ballistic impact generates a region of localized compressive stress below the projectile, and will activate the indirect tension mechanism of penetration. This would be especially important during the initial loading stages of edge-clamped laminates and throughout the impact process for laminates supported on a foundation. A high concentration of missing fiber defects would reduce the laminates transverse compressive strength, reduce the work done in penetrating a ply, and increase the laminates susceptibility to perforation. Substantial increases in ballistic performance might therefore be achieved from efforts to decrease the volume fraction of missing fiber defects. 


\subsection{References}

[1] O'Masta MR, Deshpande VS, Wadley HNG. Defect Controlled Transverse Compressive Strength of Ballistic Grade Polyethylene Fiber Laminates. Int J Solids Struct 2014:[Submitted for publication].

[2] Attwood JP, Khaderi S, Karthikeyan K, Fleck NA, O'Masta MR, Wadley HNG, et al. The out-of-plane compressive response of Dyneema composites. J Mech Phys Solids 2014:[Submitted for publication].

[3] Eitzen DG, Wadley HNG. Acoustic emission: establishing fundamentals. J Res Natl Bur Stand 1984;89:75-100.

[4] Zhuang X, Yan X. Investigation of damage mechanisms in self-reinforced polyethylene composites by acoustic emission. Compos Sci Technol 2006;66:4449.

[5] Wang X, Zhang H-P, Yan X. Classification and identification of damage mechanisms in polyethylene self-reinforced laminates by acoustic emission technique. Polym Compos 2011;32:945-59.

[6] Chiou C-P, Margetan FJ, Barnard DJ, Hsu DK, Jensen T, Eisenmann D. Nondestructive characterization of UHMWPE armor materials. AIP Conf. Proc., vol. 1430, AIP Publishing; 2012, p. 1168-75.

[7] Palka N, Miedzinska D. Detailed non-destructive evaluation of UHMWPE composites in the terahertz range. Opt Quantum Electron 2013;Online:1-11.

[8] Ho S, Suo Z. Tunneling cracks in constrained layers. J Appl Mech 1993;60:890-4.

[9] Xia ZC, Carr RR, Hutchinson JW. Transverse cracking in fiber-reinforced brittle matrix, cross-ply laminates. Acta Metall Mater 1993;41:2365-76.

[10] Barrera GD, Bruno J a. O, Barron THK, Allan NL. Negative thermal expansion. J Phys Condens Matter 2005;17:R217.

[11] Yamanaka A, Kashima T, Tsutsumi M, Ema K, Izumi Y, Nishijima S. Thermal Expansion Coefficient of Unidirectional High-Strength Polyethylene Fiber Reinforced Plastics at Low Temperature. J Compos Mater 2007;41:165-74.

[12] Greenhalgh ES, Bloodworth VM, Iannucci L, Pope D. Fractographic observations on Dyneema ${ }^{\circledR}$ composites under ballistic impact. Compos Part Appl Sci Manuf 2013;44:51-62.

[13] Freitas CJ, Bigger RP, Scott N, LaSala V, MacKiewicz J. Composite materials dynamic back face deflection characteristics during ballistic impact. J Compos Mater 2013:0021998313487934. 


\section{Chapter 6. Ballistic impact response of HB26 encased aluminum-alumina hybrid panel $\operatorname{target}^{14}$}

The indirect tension mechanism is activated by the transverse (out of plane) compressive loading of a $\left[0^{\circ} / 90^{\circ}\right]$ polymer composite due to a ply's anisotropic Poisson expansion [2]. The fibers in the plies are loaded in tension and fail when the tensile stress of the ply is reached. This mechanism was shown in Chapter 5 to be active across a range of UHMWPE fiber reinforced laminates, and predictions of the compressive behavior that use the material properties and architecture of the laminate were verified. We believe this same mechanism controls the well documented [3-8] progressive penetration of polymer composites under ballistic impact loading conditions and will discuss it in the context of the study presented in Chapter 4.

In Chapter 4, bi-material targets consisting of an aluminum alloy plate wrapped in a (Dyneema ${ }^{\circledR}$ grade HB26) UHMWPE fiber reinforced laminate were impacted with a

\footnotetext{
${ }^{14}$ A paper [1] based upon the research presented in this chapter is being prepared for submission to the International Journal of Impact Engineering.
} 
spherical projectile, Figure 4.5(a). The aluminum plate thickness was chosen to allow tests at the shatter gap threshold for the projectile/alloy combination [9], while the Dyneema $^{\circledR}$ wrapping strategy was used to avoid grip region laminate failure modes. Impact velocity dependent fragmentation of the projectile within the aluminum plate allowed the cross sectional area of the debris exiting the aluminum plate (and therefore loading the inside of the rear laminate) to be systematically varied. This enabled the cross sectional area of the laminate loading to be varied by about a factor of two, and revealed that the penetration resistance of the laminate was significantly increased as the loading area increased, Figure 4.19(b). The study also led to the discovery that out of plane bulging of the aluminum plate, prior to its perforation by the projectile within, caused the rear laminate to be accelerated away from the alloy plate back face before impact by the projectile debris, Figure 4.20. This decreased the velocity difference between the laminate and the projectile, and allowed the laminate to sustain even higher velocity impacts in comparison to cases where the rear laminate was stationary upon impact.

In an impact event, a region of compression develops in a laminate under the projectile. The magnitude of this pressure scales with impact velocity, as well as the shape, density and sound speed of the projectile and its angle of impact $[10,11]$. If the impact produces a compressive stress that is sufficiently high, it will cause indirect tensile fracture of a ply's fibers. This suggests that any method that reduces the contact pressure applied to the laminate by a projectile should delay the onset of the indirect tension failure mode to higher impact velocities.

Data compiled for several fiber reinforced laminate systems does indeed show that the ballistic limit increases with the ratio of the projectile impact area to projectile 
mass [12]. Karthikeyan et al. [13] also observed that when edge clamped UHMWPE fiber reinforced Dyneema ${ }^{\circledR}$ HB26 cross-ply laminates were impacted by large cross sectional area (but low density) metal foam projectiles, penetration could be suppressed, and the impact energies sustained by the laminate were much greater than those for a localized impact by a solid projectile of similar mass $[8,13]$. In the metal foam impact experiments, the location of failure was also observed to move from the impact site (for solid projectiles) to the grips, and novel gripping strategies that avoided creating bolt holes in the laminate substantially improved the failure impulse threshold.

Several studies of the impact of hybrid sandwich panel structures whose cores contained both hard ceramic, and ductile metallic materials have revealed interesting opportunities to tune the impact debris spatial distribution, and potentially applying substantial acceleration to the rear face of the structure during an impact event [14-18]. In a recent study of a model aluminum sandwich panel, aluminum corrugations were filled with prismatic, triangular cross section alumina inserts, and impacted above either a prism base or a prism apex, Figure 6.1 [17]. The rear aluminum face sheet was observed to suffer a large out of plane deflection prior to projectile and target debris ejection. The width of deflected region and debris plume were found to be controlled by the impact location on the front face of the target, and the base width of the corrugated cells, $\mathrm{L}_{\mathrm{c}}$. Impacts above the base of a prism resulted in debris clouds whose width was $\sim 2 \mathrm{~L}_{c}$, or four times that of the impacting projectile (whose diameter was about $0.5 \mathrm{~L}_{\mathrm{c}}$ ), Figure 6.1(a). However impacts above a prism apex (a corrugation node), Figure 6.1(b), resulted in an out of plane deflection width of $\sim \mathrm{L}_{\mathrm{c}}$, and with a much increased debris exit velocity. 


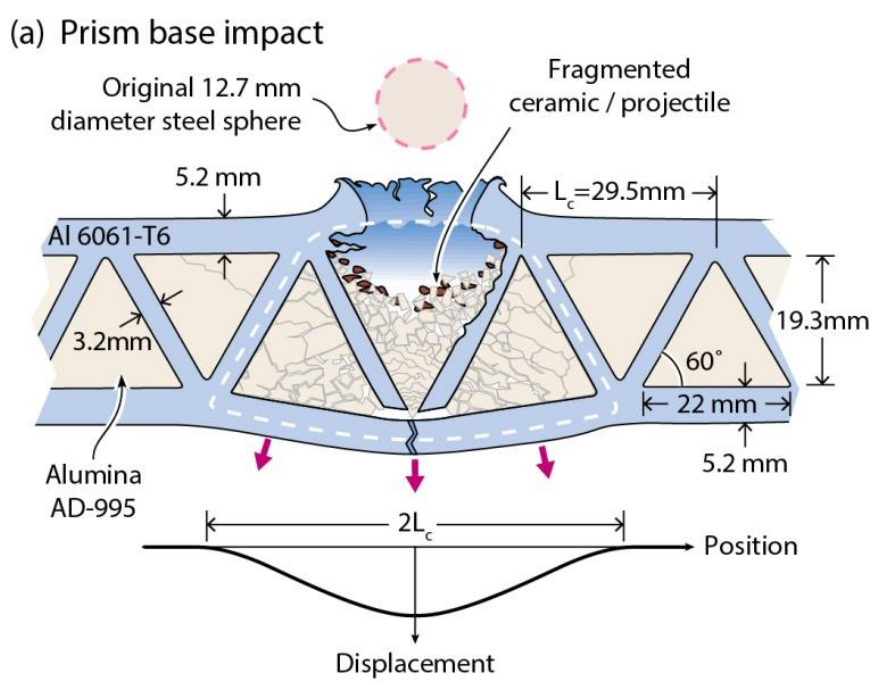

(b) Prism apex impact

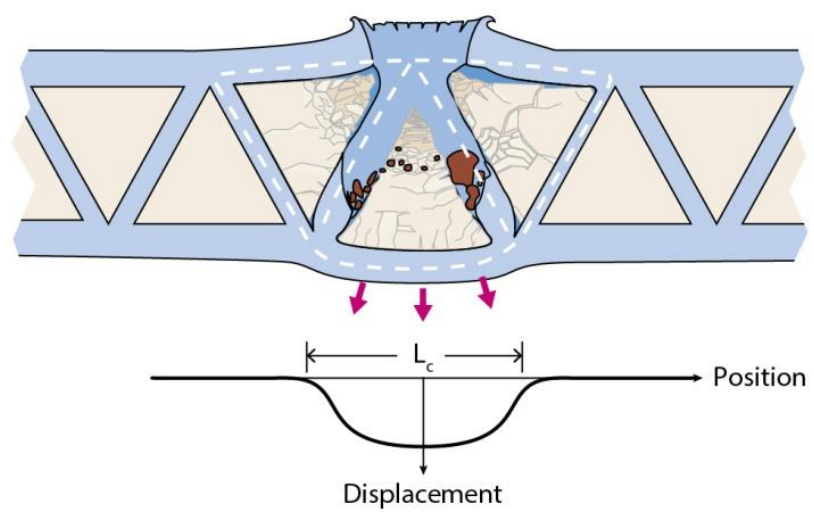

Figure 6.1. Schematic illustration of a corrugated aluminum sandwich structure with an alumina filled (hybrid) core after impact by a hardened steel sphere. The length of the out of plane displaced region on the rear face depended upon impact location. (a) A ceramic prism base impact resulted in a region of displacement $2 L_{c}$ wide, while $(b)$ shows that an impact at the apex of a prism led to a displacement of half this width.

Repeating such an experiment with a hybrid core test structure encased in a crossply UHMWPE fiber reinforced laminate would allow a further assessment of the consequences of changing the rear laminate contact pressure. Here, the HB26 laminate encapsulated an aluminum alloy sandwich panel structure whose corrugated core was filled with prismatic alumina inserts. The laminate encased hybrid core target could sustain ceramic prism base impacts by a spherical, $12.7 \mathrm{~mm}$ diameter steel projectile with velocities in excess of $2.7 \mathrm{~km} \mathrm{~s}^{-1}$. This was $150 \%$ higher than the ballistic limit of the 
equal areal density encapsulated aluminum plate target constructed with the same laminate. Using high-speed imaging, three-dimensional digital image correlation, $\mathrm{x}$-ray tomography and a modification to the encased hybrid target design, the enhanced response is shown to be due to a redistribution of the impact pressure field and preacceleration of the laminate by the rear face of the deforming sandwich panel prior to laminate impact by the fragmented projectile and ceramic debris. The rear laminate is shown to sustain impacts by the released debris in excess of $600 \mathrm{~m} \mathrm{~s}^{-1}$ without penetration by the debris particles. The kinetic energy of the impact was then dissipated by large membrane mode deflections of the laminate.

\subsection{Sample fabrication}

The model target design is shown in Figure 6.2. The samples were fabricated using the same $5.9 \mathrm{~mm}$ thick Dyneema ${ }^{\circledR}$ HB26 fiber-reinforced laminate used in Chapter 4. It encased a slightly modified version of the same hybrid core sandwich panel studied by Wadley et al. [17]. In the original Wadley et al. study, the 6061-T6 aluminum alloy sandwich panel had a triangular corrugated core with $3.2 \mathrm{~mm}$ thick webs inclined at an angle of $60^{\circ}$ to two, $5.2 \mathrm{~mm}$ thick face sheets, Figure 6.1. In the present study, the thickness of the face sheet on the impact side of the corrugated panel was reduced to 1.0 $\mathrm{mm}$, Figure 6.3(a). With this modification, a hybrid core panel with a $5.9 \mathrm{~mm}$ thick Dyneema ${ }^{\circledR}$ HB26 laminate encasement had a mass per unit area of $\rho_{\mathrm{a}}=97 \mathrm{~kg} \mathrm{~m}^{-2}$; the same as the original (non-encased) hybrid panel and the encased aluminum plate target. 
(a) Encased hybrid

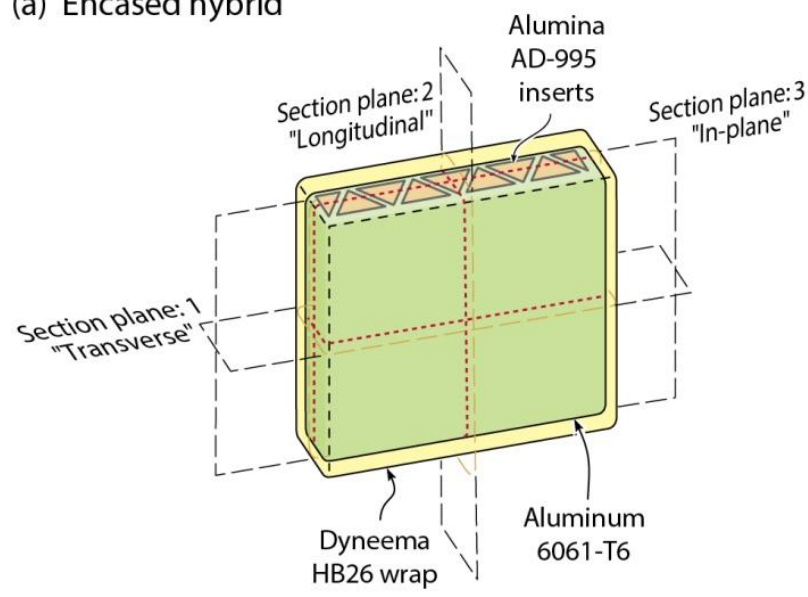

(c) Rear face cutout

(b) Encased hybrid cross-section
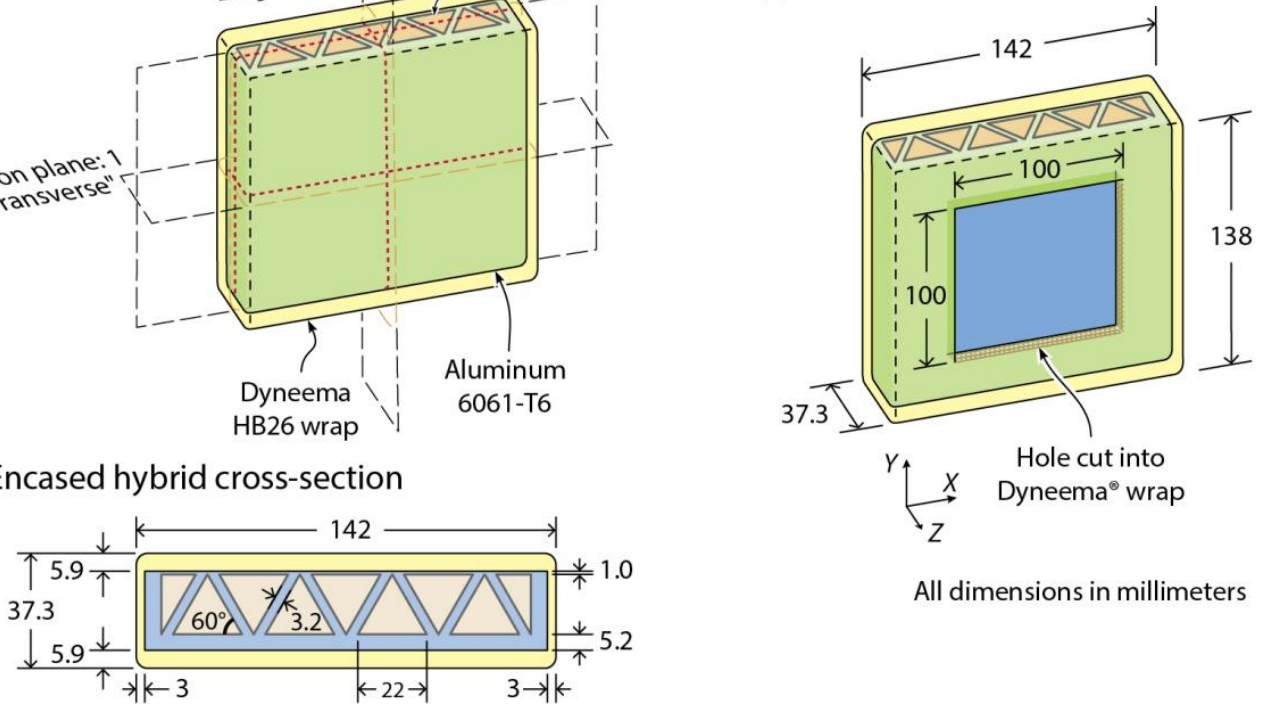

All dimensions in millimeters

Figure 6.2. Schematic illustrations of the two target types investigated in this study. (a) Shows the fully Dyneema ${ }^{\circledR}$ laminate encased sample design with dimensions defined in $(b)$. The sample design with a part of the back laminate removed is shown in (c) together with a coordinate system. Reference planes for subsequent analysis are also shown in (a).

The model targets were assembled by filling the empty cells of the sandwich panel with CoorsTek (Golden, CO) grade AD-995 triangular prisms that had been coated in Lord (Cary, NC) grade 305 epoxy adhesive, Figure 6.3(b). This ceramic has a hardness of $14.1 \mathrm{GPa}$, an elastic modulus of $370 \mathrm{GPa}$ and a fracture toughness of $4-5 \mathrm{MN} \mathrm{m}^{-3 / 2}$. The panels were encased in Dyneema ${ }^{\circledR}$ grade HB26 cross-ply laminate, whose properties are provided in Section 2.2. The procedure in Section 4.1.2 to apply the pre-preg and consolidate the laminate was followed and is illustrated in Figure 6.3(c-d). Again the layup of the laminate on both $136 \mathrm{~mm} \times 132 \mathrm{~mm}$ faces was $\left[\left(90^{\circ} / 0^{\circ}\right)_{2} /\left(0^{\circ} / 90^{\circ}\right)_{2}\right]_{11}$ with a thickness of $5.9 \mathrm{~mm}$, which was twice the thickness of the four $\left[\left(0^{\circ} / 90^{\circ}\right)\right]_{22}$ sides. The length of applied pre-preg material per strip was $\sim 5 \mathrm{~cm}$ longer than calculated for a perfectly tight wrap. The excess (but densified) Dyneema ${ }^{\circledR}$ accumulated around the periphery of the top (subsequently impacted) surface of the panels. The final dimensions 
of the encased hybrid target are given in Figure 6.2. Figure 6.2(a) also identifies reference planes, while Figure 6.2(c) defines sample external dimensions and a coordinate system used later.
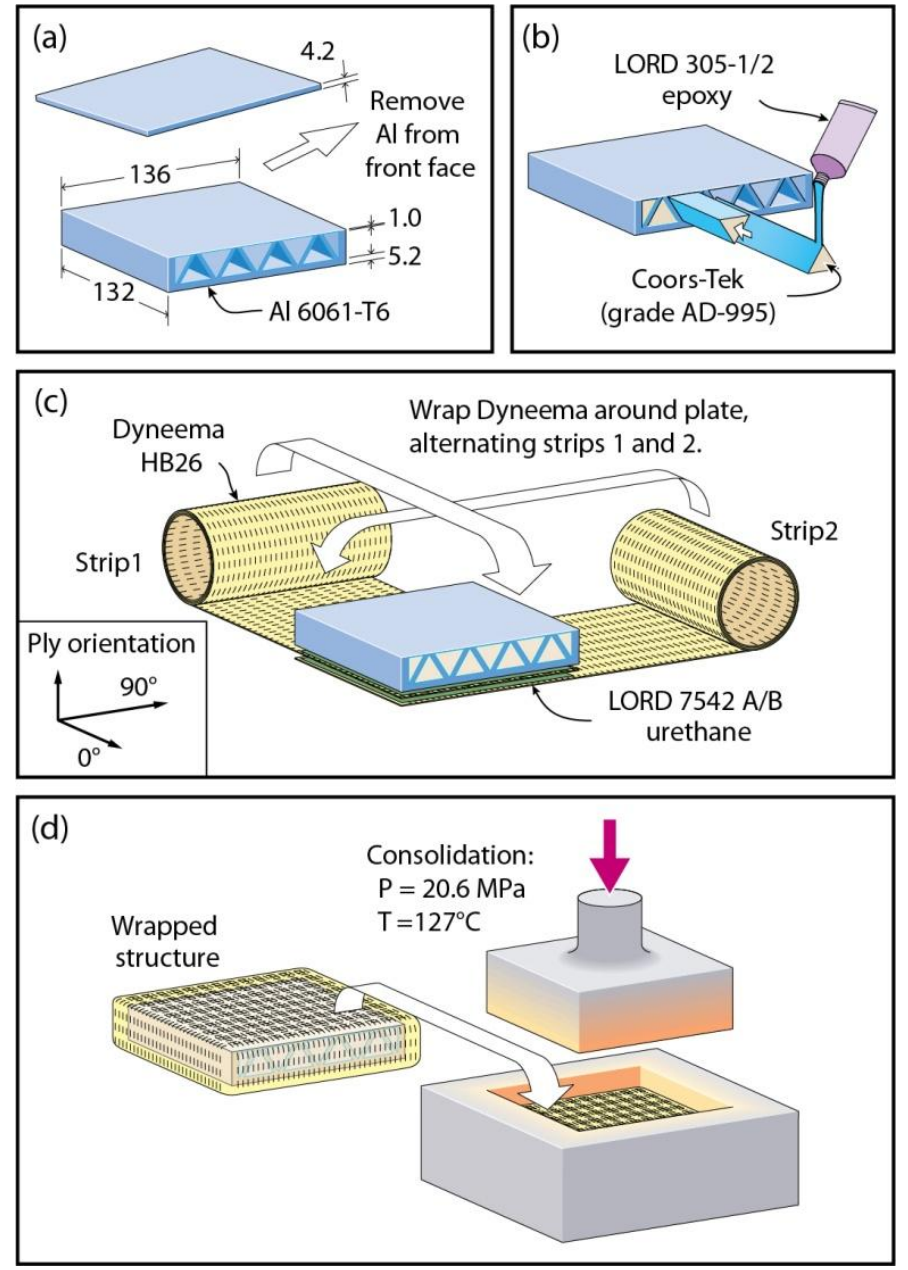

Figure 6.3. The fabrication sequence for making the Dyneema HB26 encased hybrid core panels.

To observe the hybrid sandwich panel's back face deflection, and the debris plume that is incident upon the inside surface of the rear Dyneema ${ }^{\circledR}$ laminate during an impact event, a central 100 mm x 100 mm square region of Dyneema ${ }^{\circledR}$ was removed, using a rotary cutting tool. A speckle pattern was applied to the aluminum surface to enable measurement of the surface displacement of the back surface with a three- 
dimensional digital image correlation (DIC) system. The exposed aluminum surface was first spray painted white, followed by the application of a stochastic pattern of $1-3 \mathrm{~mm}$ diameter black dots that covered approximately $50 \%$ of the aluminum surface area.

\subsection{Impact tests and characterization}

The same $12.7 \mathrm{~mm}$ diameter, 52100 hardened steel, spherical projectile used in the earlier study of the encased aluminum plate (Chapter 4) and the bare hybrid core $[19,17]$ was used here. Each target was edge clamped, and oriented for $0^{\circ}$ obliquity impact either at the mid-span of the $22 \mathrm{~mm}$ wide base of the center ceramic prism, Figure 6.4 , or on the apex of one of the adjacent ceramic prisms. The impact tests on the fully encased hybrid target were performed with a light-gas gun capable of launch velocities up to $3.5 \mathrm{~km} \mathrm{~s}^{-1}$ located at the University of California, Santa Barbara [18]. The impact velocity, $\mathrm{Vi}$, was measured to within $\pm 0.4 \%$ using the time-of-flight recorded between a pair of laser-gates. A model v.7.3 Phantom (Vision Research Inc.; Wayne, NJ) highspeed video camera was oriented normal to the $\mathrm{YZ}$ plane, and recorded a backlit profile view of the rear of the sample. The camera recordings used an exposure time of $1 \mu$ s and an inter-frame interval of $19.5 \mu \mathrm{s}$. After impact, the samples were visually inspected for external damage. Some samples were examined by x-ray computed tomography (XCT) at Carl Zeiss Imaging (Brighton, MI). All of the samples were then sectioned by water-jet cutting along the transverse plane that intersected the impact sites. 


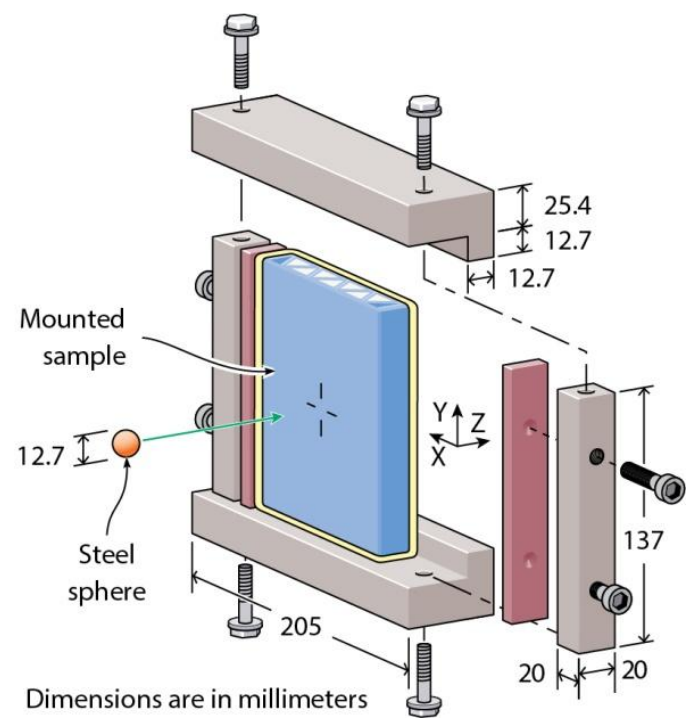

Figure 6.4. Schematic illustration of the ballistic test fixture and sample support configuration.

The ballistic impacts of the rear face cutout targets were performed at Chesapeake Testing (Belcamp, MD) where the more open geometry enabled 3D-DIC measurements to be recorded. The impact velocities achievable with this powder gun were limited to $V_{i}$ $<2.3 \mathrm{~km} \mathrm{~s}^{-1}$ and were measured using paper break-screens. The Z-component of the residual velocity, $V_{r}$, of the leading edge of the debris released from perforated samples was calculated from video images obtained with a model v.1610 Phantom high-speed camera oriented normal to the $\mathrm{YZ}$ plane. The recordings used an exposure time of $0.76 \mu \mathrm{s}$ and an inter-frame interval of $10 \mu \mathrm{s}$. A pair of model Fastcam SA-X2 Photron (San Diego, CA) high-speed cameras were positioned in a stereo configuration $\left(\alpha=17.5^{\circ}\right)$ behind the sample to record the deflection of the speckle pattern coated rear aluminum surface, Figure 6.5. Each black dot filled approximately 6 pixels of a camera's field of view. The cameras were positioned to the side of the Z-axis to avoid damage from debris impact, and were synchronized to capture images at $6.67 \mu$ s intervals with an exposure time of $1 \mu \mathrm{s}$. 


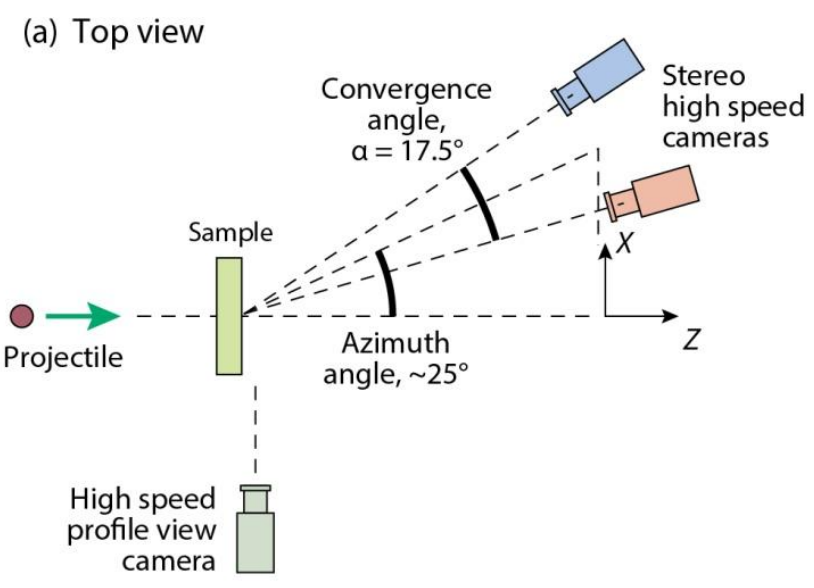

(b) Rear perspective view

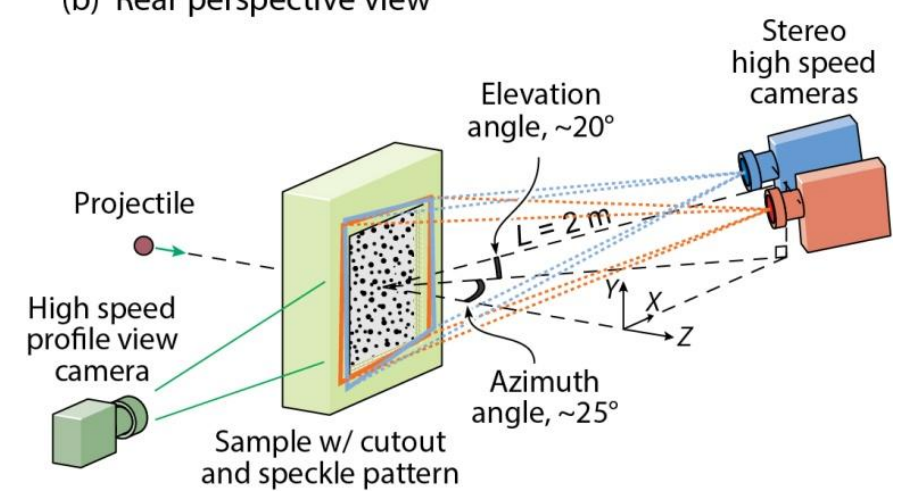

Figure 6.5. Schematic illustrations showing (a) the top and (b) the rear perspective views of the impact test geometry used to record $3 D$ DIC measurements.

The DIC images were analyzed using the Aramis v.6.3 3D image correlation software reviewed in Section 3.5.1. Presently, a facet area of 15 pixel x 15 pixel was used, and each linear raster of facets was spaced 5 pixels $(\sim 1.8 \mathrm{~mm})$ apart. Additional details of high-speed DIC acquisition and analysis can be found in references [20-22].

\subsection{Results}

\subsubsection{Encased hybrid targets - ceramic prism base impacts}

The majority of the fully encased targets were impacted at a prism base, mid-way along the prism at impact velocities between 0.84 and $2.70 \mathrm{~km} \mathrm{~s}^{-1}$, while one sample was 
impacted on a prism apex at a velocity of $2.5 \mathrm{~km} \mathrm{~s}^{-1}$. Table 6.1 summarizes the impact and residual (exit) velocities for these experiments. The projectiles that impacted at a prism base were arrested within the samples without ejection of debris, while the impact on a prism apex resulted in full perforation with a debris exit velocity of $0.2 \mathrm{~km} \mathrm{~s}^{-1}$. Table 6.2 summarizes the ballistic limits measured previously for the encased aluminum and the hybrid core reference targets of identical aerial density $[19,17]$. It is evident that the ballistic limit of the encased hybrid target tested here was at least double that of the reference targets. 
Table 6.1. Impact results for the encased hybrid and rear face cutout targets

\begin{tabular}{l|lrrr}
\hline \multicolumn{1}{c}{ Target type } & $\begin{array}{c}\text { Impact } \\
\text { location }\end{array}$ & $\begin{array}{c}V_{i} \\
\left(\mathrm{~km} \mathrm{~s}^{-1}\right)\end{array}$ & $\begin{array}{c}\dot{\delta}_{\text {max }} \\
\left(\mathrm{km} \mathrm{s}^{-1}\right)\end{array}$ & $\begin{array}{r}V_{r} \\
\left(\mathrm{~km} \mathrm{~s}^{-1}\right)\end{array}$ \\
\hline Encased hybrid & Base & 0.84 & $\mathrm{~N} / \mathrm{R}$ & 0 \\
& Base & 1.00 & $\mathrm{~N} / \mathrm{R}$ & 0 \\
& Base & 1.34 & $\mathrm{~N} / \mathrm{R}$ & 0 \\
& Base & 1.48 & $\mathrm{~N} / \mathrm{R}$ & 0 \\
& Base & 2.00 & $\mathrm{~N} / \mathrm{R}$ & 0 \\
& Base & 2.15 & $\mathrm{~N} / \mathrm{R}$ & 0 \\
& Base & 2.26 & $\mathrm{~N} / \mathrm{R}$ & 0 \\
& Base & 2.31 & $\mathrm{~N} / \mathrm{R}$ & 0 \\
& Node & 2.50 & $\mathrm{~N} / \mathrm{R}$ & 0.20 \\
& Base & 2.57 & $\mathrm{~N} / \mathrm{R}$ & 0 \\
& Base & 2.70 & $\mathrm{~N} / \mathrm{R}$ & 0 \\
\hline & Base & 1.71 & 0.32 & 0.43 \\
& Base & 1.84 & 0.34 & 0.50 \\
& Base & 1.92 & 0.35 & 0.49 \\
& Node & 2.00 & 0.40 & 0.82 \\
& Node & 2.17 & 0.45 & 0.80 \\
& Base & 2.29 & 0.45 & 0.62 \\
\hline
\end{tabular}

N/R: Not Recorded

Table 6.2. The ballistic limits of reference targets $\left(\rho_{a}=97 \mathrm{~kg} \mathrm{~m}^{-2}\right)$ presented by the highest impact velocity that failed to completely perforate the target and the lowest impact velocity that did.

\begin{tabular}{llcc}
\hline & Impact & \multicolumn{2}{c}{ Ballistic limit $\left(\mathrm{km} \mathrm{s}^{-1}\right)$} \\
\cline { 3 - 4 } Target type & location & Not perforated & Perforated \\
& & & \\
\hline Encased Al & - & 1.37 & 1.41 \\
Hybrid & Base & 1.27 & 1.32 \\
Hybrid & Node & 0.98 & 1.15 \\
\hline
\end{tabular}

Transverse cross-sections of several of the encased hybrid core targets impacted at a prism base are shown in Figure 6.6. The front laminate was completely perforated by an impact at $0.84 \mathrm{~km} \mathrm{~s}^{-1}$, Figure 6.6(a). This was consistent with a previous measurement 
of $0.20 \mathrm{~km} \mathrm{~s}^{-1}$ for the ballistic limit of a $5.9 \mathrm{~mm}$ thick HB26 laminate supported on a foundation and impacted by the same projectile, Table 6.3 [19]. The projectile then perforated the $1 \mathrm{~mm}$ thick front face sheet and was arrested within the impacted ceramic prism which suffered substantial comminution. The hole seen in the impacted cell resulted from reverse flow of the comminuted ceramic and projectile fragments through the entry hole during impact, and loss of the remaining material during cross-sectioning. Some microcracking of the two prisms on either side of the impact can also be seen in Figure 6.6(a). It is interesting to note that the Dyneema ${ }^{\circledR}$ entry hole had partially closed after impact, limiting the exit of debris through the front face. The target suffered minimal permanent back face deflection. 

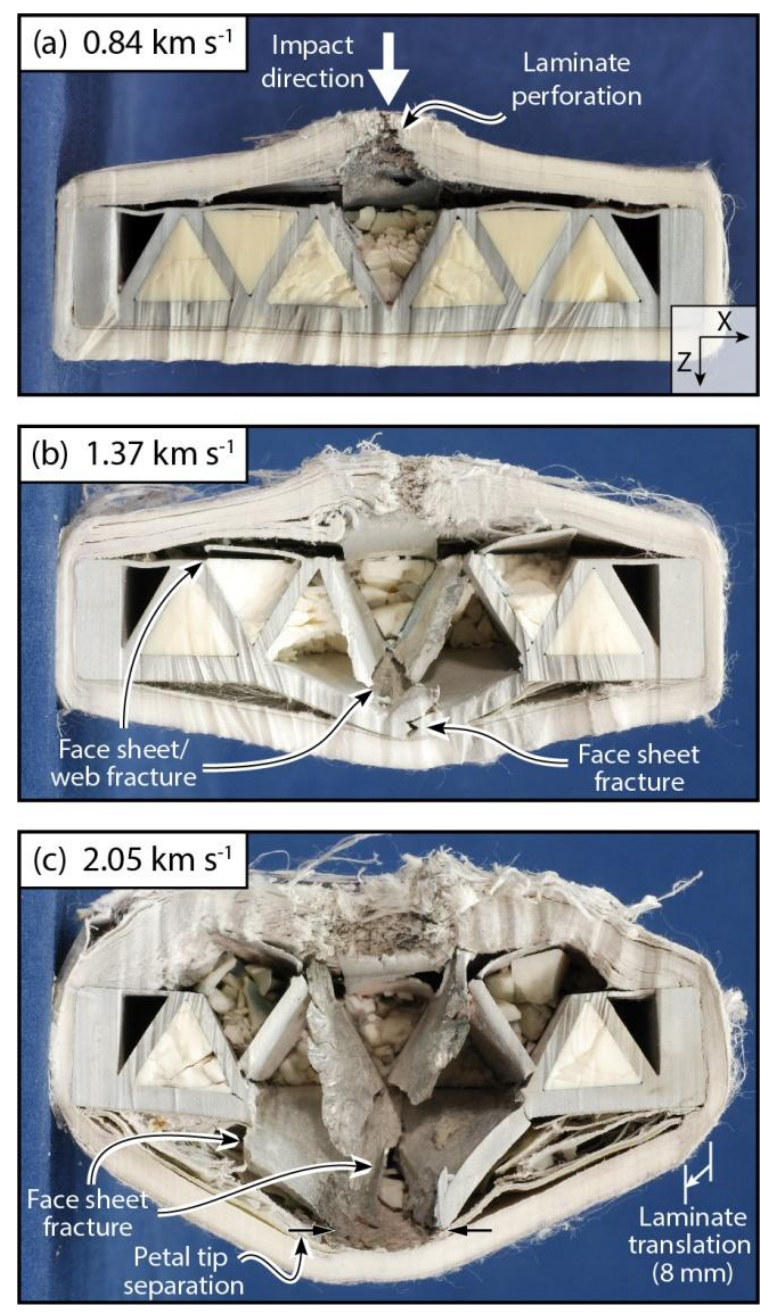

Figure 6.6. Transverse cross-sections of Dyneema ${ }^{\circledR}$ encased hybrid targets impacted by the 12.7 mm diameter hardened steel sphere on a prism base. Wavy vertical lines are an artifact of the water-jet sectioning process.

Table 6.3. The minimum impact velocity for penetration (first ply failure) and perforation (complete laminate failure) of $5.9 \mathrm{~mm}$ thick HB26 laminates when impacted by a $12.7 \mathrm{~mm}$ diameter steel sphere.

\begin{tabular}{llcc}
\hline & & \multicolumn{2}{c}{ Velocity $\left(\mathrm{km} \mathrm{s}^{-1}\right)$} \\
\cline { 3 - 4 } Target & Support condition & Penetration & Perforation \\
\hline $\begin{array}{l}\text { Encased aluminum } \\
\text { (Chapter 4) }\end{array}$ & Rear supported & $<0.11$ & 0.20 \\
$\begin{array}{l}\text { Encased aluminum } \\
\text { (Chapter 4) }\end{array}$ & $\begin{array}{l}\text { Edge clamped }+ \\
\text { pre-acceleration }\end{array}$ & 0.24 & 0.60 \\
Bare laminate plate [8] & \begin{tabular}{l} 
Edge clamped \\
\hline
\end{tabular} & 0.24 & 0.45 \\
\hline
\end{tabular}


The effect of increasing the impact velocity can be seen in Figure 6.6(b) and (c). The impact at $1.37 \mathrm{~km} \mathrm{~s}^{-1}$ displaced a wide section of the rear face sheet in the Zdirection; sufficient to cause fracture of the webs of the aluminum corrugated core and the rear face sheet. This resulted in substantial permanent deflection, but no penetration of the rear laminate. The length of the displaced face sheet region was approximately $50 \mathrm{~mm}$, or $\sim 2 \mathrm{~L}_{\mathrm{c}}$, in the $\mathrm{X}$-direction. XCT results for this sample, Figure 6.7(a), show that the length of the displaced face sheet region in the $\mathrm{Y}$-(prism axis) direction was about $75 \mathrm{~mm}$. The position of the hinges about which face sheet deflection occurred, coincided with the base of the cone shaped region of severely microcracked ceramic, Figure 6.6(b) and Figure 6.7. For impacts with $V_{i} \geq 1.48 \mathrm{~km} \mathrm{~s}^{-1}$, the rear face sheet was longitudinally torn directly beneath the impact site and at the two adjacent nodes in the X-direction, Figure 6.6(c). Figure 6.6 also shows that the hole on the front face increased in width as more substantial reverse debris flow eroded the sides of the entry hole. 

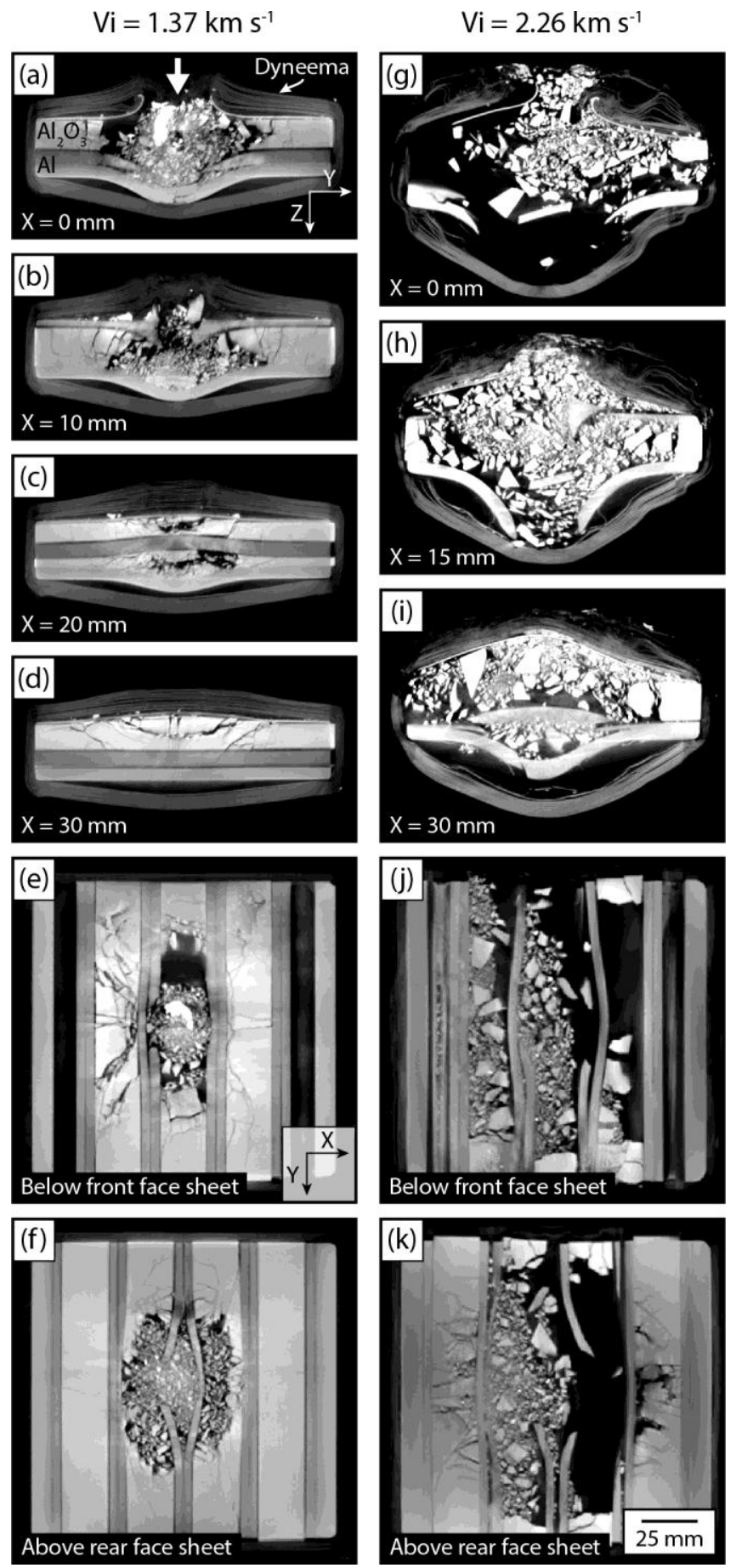

Figure 6.7. XCT reconstructions of longitudinal ( $a-d$ and $g-i)$ and in-plane ( $e, f, j$ and $k$ ) crosssectional renderings of encased hybrid targets impacted at $V_{i}=1.37 \mathrm{~km} \mathrm{~s}^{-1}\left(\right.$ a-f), and $V_{i}=2.26$ $\mathrm{km} \mathrm{s}^{-1}(\mathrm{~g}-\mathrm{k})$. The arrow in (a) indicates impact direction for both samples.

The displacement of the rear aluminum face sheet caused a permanent displacement of the rear Dyneema ${ }^{\circledR}$ laminate by a distance that increased with impact 
velocity, Figure 6.6 and Figure 6.7. At $V_{i}=2.05 \mathrm{~km} \mathrm{~s}^{-1}$, rotation of the fractured rear face sheet cut four to six of the Dyneema ${ }^{\circledR}$ plies and allowed the comminuted ceramic and fragmented projectile debris to impact the laminate. However, this debris impact appears to have caused no additional failure of the rear laminate. The large stretching displacement suffered by the rear laminate appears to have been accommodated by pullin of plies from the sides and front of the encasement. At the highest test velocity $\left(2.7 \mathrm{~km} \mathrm{~s}^{-1}\right)$, portions of the laminate encasement at the sides of the sample began to fail, Figure 6.8(a). No local penetration of the laminate at the impact location was observed in any of the prism base impact experiments. 

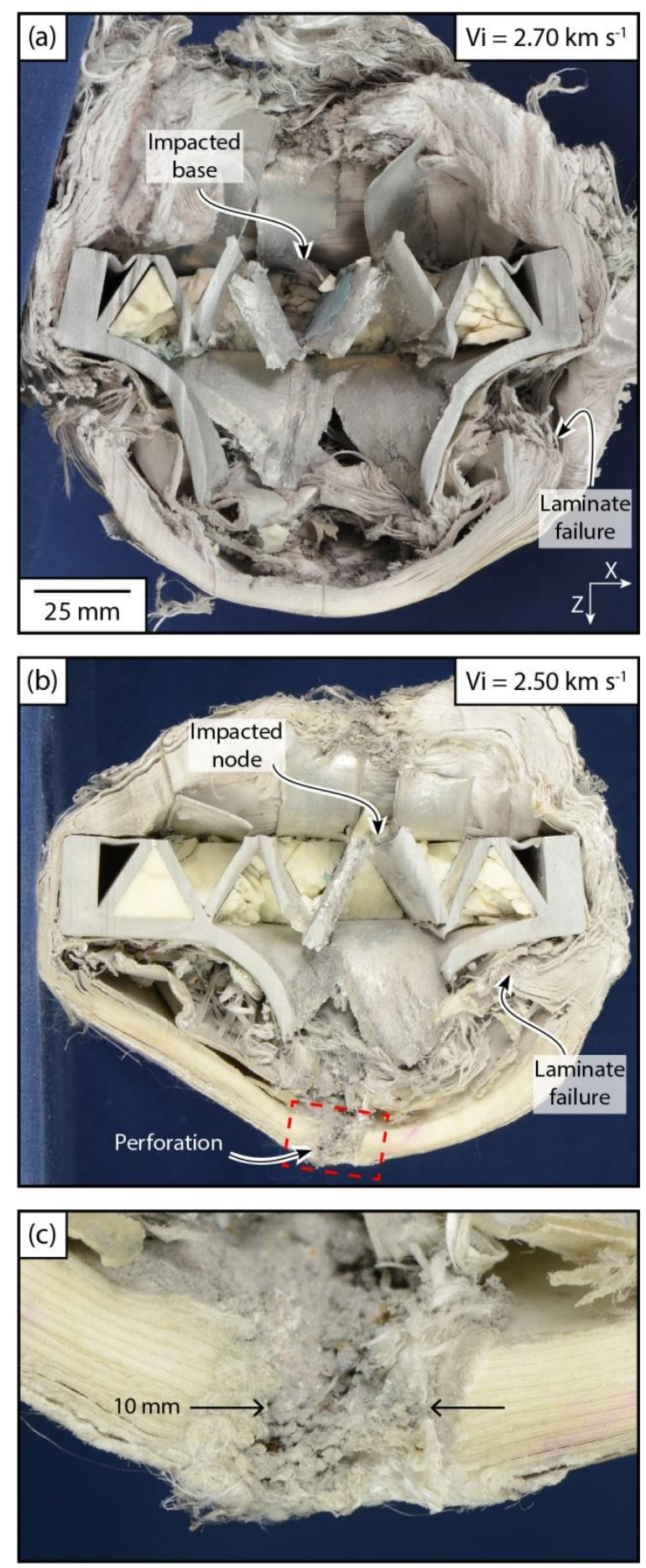

Figure 6.8. Transverse cross-sections of encased hybrid targets impacted at either (a) a prism base or (b) a prism apex. Prominent laminate failure mechanisms are identified. (c) Magnified view of the perforated region of the laminate shown in $(b)$. 
A high-speed image sequence showing the back face profile of the encased hybrid target impacted at $2.70 \mathrm{~km} \mathrm{~s}^{-1}$ is shown in Figure 6.9. At $t=15 \mu \mathrm{s}^{15}$, the laminate on the rear of the sample had been displaced $12 \mathrm{~mm}$ in the Z-direction and formed a $58 \mathrm{~mm}$ wide bulge in the Y-direction, consistent with acceleration by a bulging rear aluminum face sheet. The initial out of plane (Z-component) velocity was $0.5 \mathrm{~km} \mathrm{~s}^{-1}$, and the distance of the laminate's Z- component deflection increased with time as the aluminum face sheet was fractured and released debris against the laminate. The laminate deflection was accommodated by transverse hinges that propagated away from the impact site with a Y-component velocity of $0.32 \pm 0.03 \mathrm{~km} \mathrm{~s}^{-1}$; identical to that measured for encased aluminum samples. The finger-like protrusions visible at $t \geq 141 \mu \mathrm{s}$, were delaminated Xoriented fibers that had failed at a side grip. The side edge of the outermost tape strip is highlighted in the $t=366 \mu$ s image. This edge feature was a result of the combined pullin and tensile rupture of laminate material, as previously shown in Figure 6.8(a). The impact was arrested within $600 \mu \mathrm{s}$, after a maximum peak deflection of $82 \mathrm{~mm}$.

\footnotetext{
${ }^{15}$ Time $t=0 \pm 4 \mu \mathrm{s}$ was the estimated time of the start of deflection. Note this is different than defined in Chapter 4, where $t=0 \mu$ s was the estimate of target impact.
} 

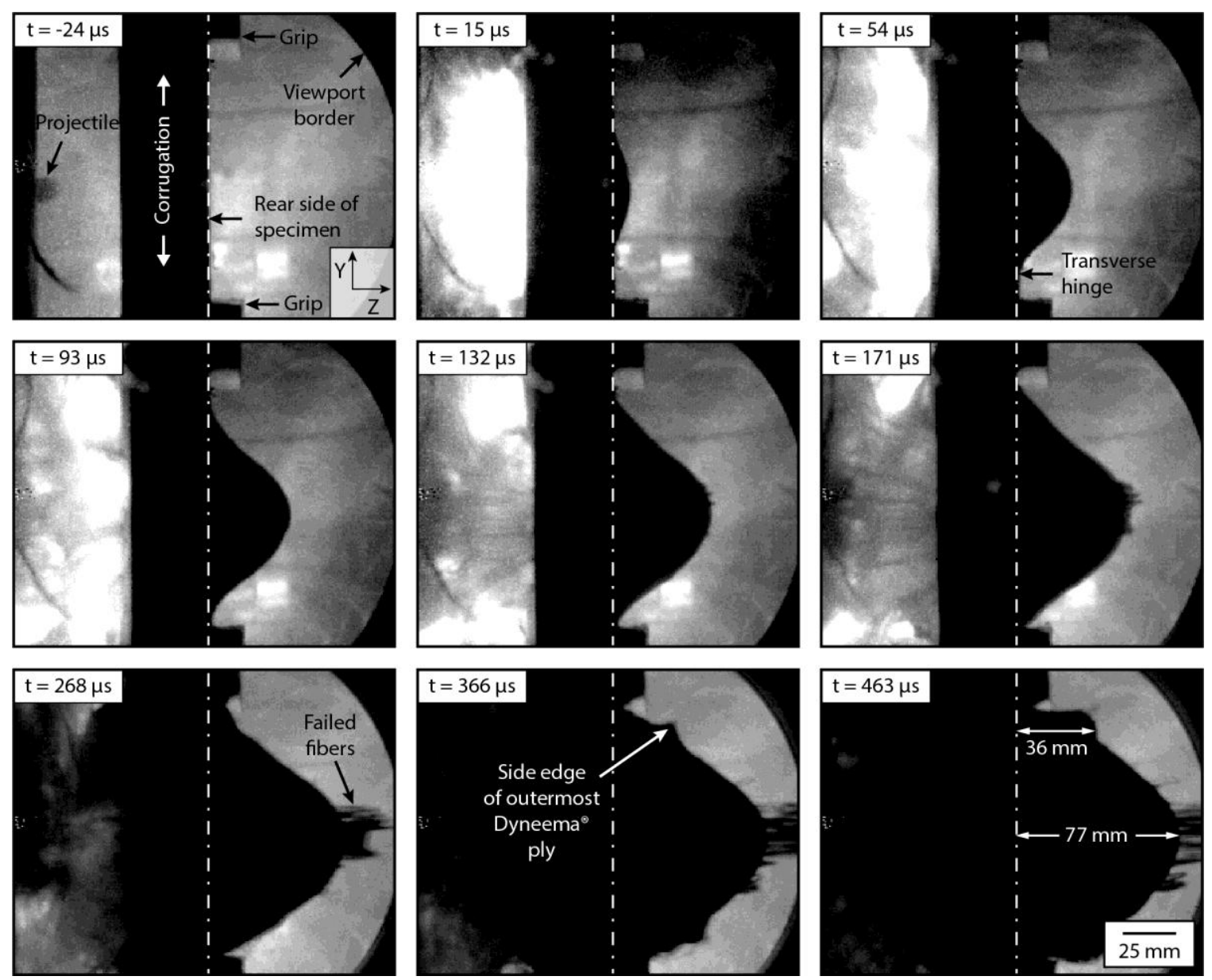

Figure 6.9. High-speed video image sequence of an encased hybrid target during prism base impact at $V_{i}=2.70 \mathrm{~km} \mathrm{~s}^{-1}$.

\subsubsection{Encased hybrid targets - ceramic prism apex impacts}

A cross-sectional view of the encased hybrid core target impacted at the apex of the ceramic prism at $V_{i}=2.50 \mathrm{~km} \mathrm{~s}^{-1}$ is shown in Figure $6.8(\mathrm{~b})$. Like a base impact at this impact velocity, the rear laminate transversely deflected, pulled material in from the sides and portions failed in tension along the sides. However in the apex impact case, the laminate was perforated below the impact site leaving a $\sim 10 \mathrm{~mm}$ diameter hole, Figure $6.8(c)$ 


\subsubsection{Rear face cutout targets - ceramic prism base impacts}

The rear face cutout targets were impacted at a prism base at velocities of 1.71 to $2.29 \mathrm{~km} \mathrm{~s}^{-1}$, Table 6.1. As with the encased hybrid targets, the projectile first perforated the front laminate and the thin aluminum front face sheet followed by fragmentation against the base of the center ceramic prism and activation of hybrid core failure mechanisms. For the $1.71 \mathrm{~km} \mathrm{~s}^{-1}$ impact, the high-speed video image sequence from one of the oblique rear view cameras of the speckle coated rear face sheet is shown in Figure 6.10(a). A contour map of the out of plane (Z-component) velocity, $\dot{\delta}$, determined from DIC analysis is overlaid. At $t=4 \mu \mathrm{s}$, a small bulge formed on the rear face sheet. Longitudinal and transverse displacement profiles across the peak of the bulge revealed it was longer in the longitudinal direction (Y-axis) than the transverse direction (X-axis), Figure 6.11(a) and (b). The dimensions of the bulge and its velocity increased with time, and a Y-oriented tear in the face sheet ${ }^{16}$ started to develop below the node of the centrally impacted cell by $t=24 \mu \mathrm{s}$ when the peak deflection reached about $6 \mathrm{~mm}$. Ejecta then exited from the tear, as seen in the last frame of Figure 6.10(a), and from the profile view of the impact in Figure 6.10 (b). The plume of debris had a blunt front and was $\sim 44 \mathrm{~mm}$ wide in the Y-direction, which is consistent with the dimension of the face sheet tear opening, Figure 6.7 and Figure 6.11(b), and the dynamic rear laminate deflection profile, Figure 6.9. At the fastest tested impact velocity, $V_{i}=2.29 \mathrm{~km} \mathrm{~s}^{-1}$, the shape of the bulge along the longitudinal plane was similar to the lower impact velocity, but the transverse profile was more localized, Figure 6.11(c) and (d).

\footnotetext{
${ }^{16}$ There were no DIC facets located along a tear since the fracture surface changed the identifying "pattern" within a facet's interrogation area.
} 
(a) Oblique rear view with velocity overlay
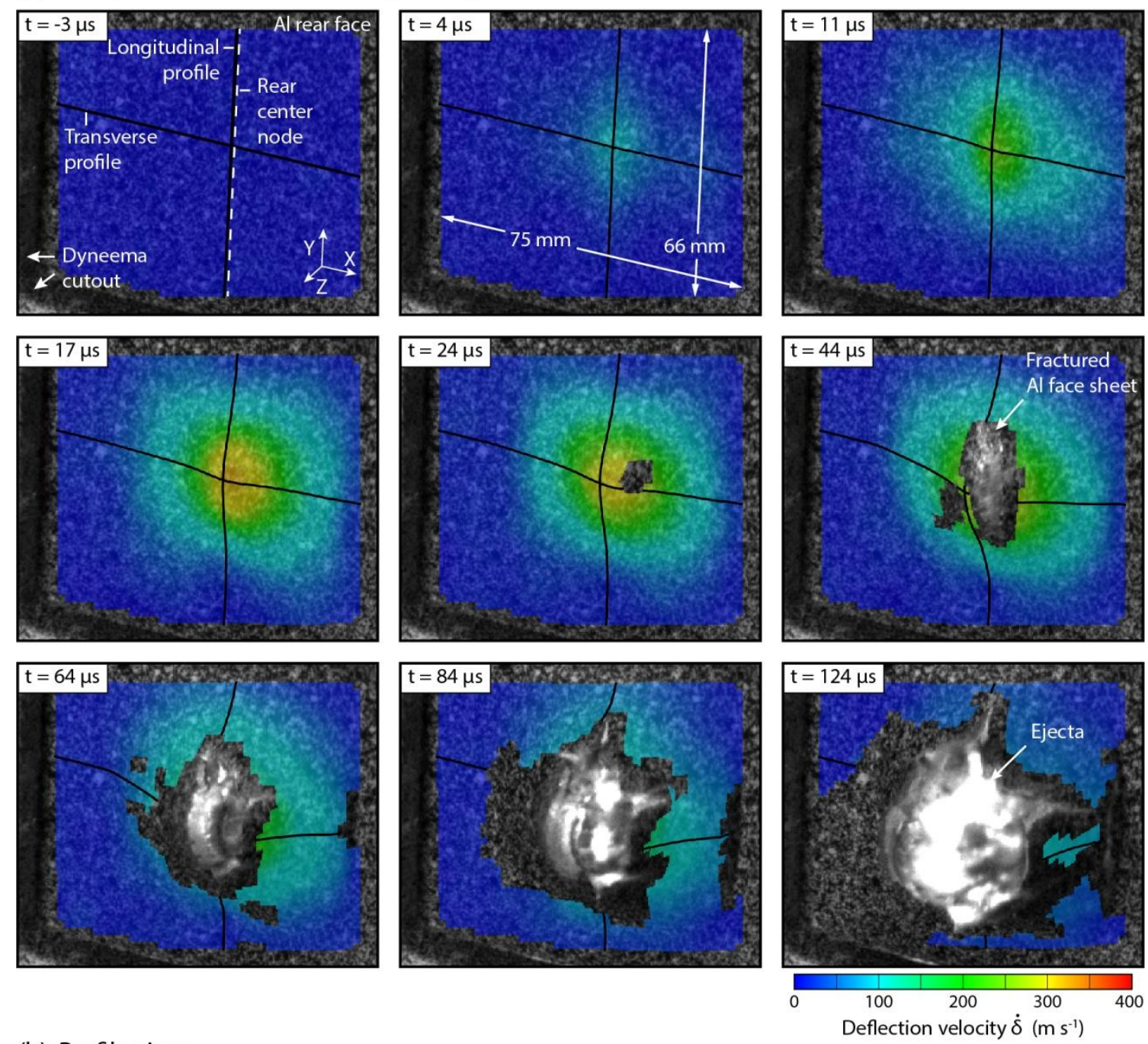

(b) Profile view
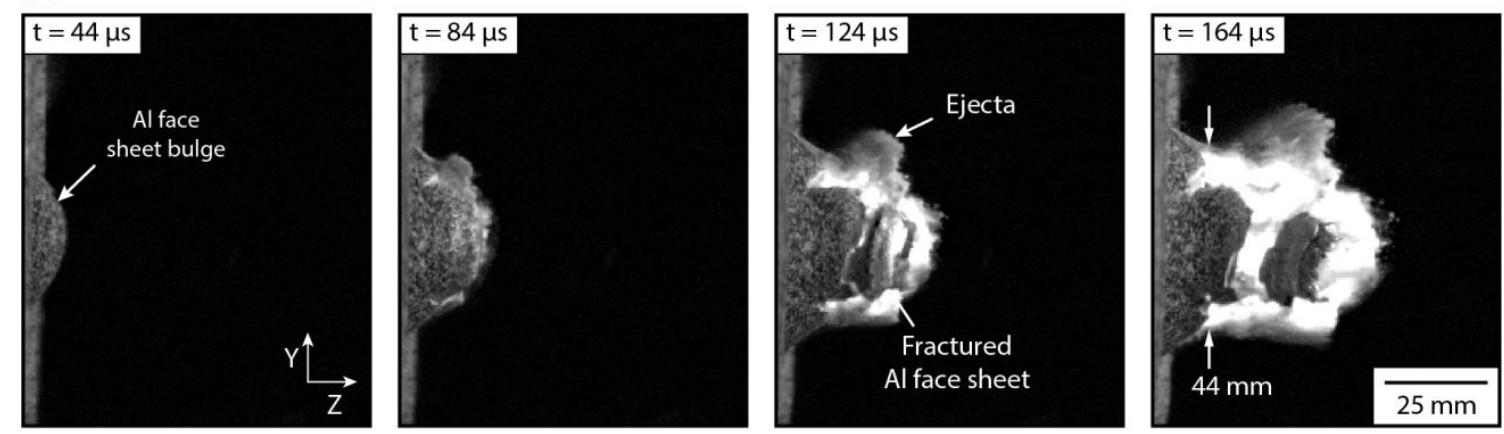

Figure 6.10. (a) High-speed video image sequence showing an oblique rear view of the back aluminum surface of a rear cutout target that was prism base impacted at $V_{i}=1.71 \mathrm{~km} \mathrm{~s}^{-1}$. A map of the out of plane surface velocity, $\dot{\delta}$, determined from DIC measurements is superimposed. (b) High speed video images showing the out of plane deflection during sample impact. 

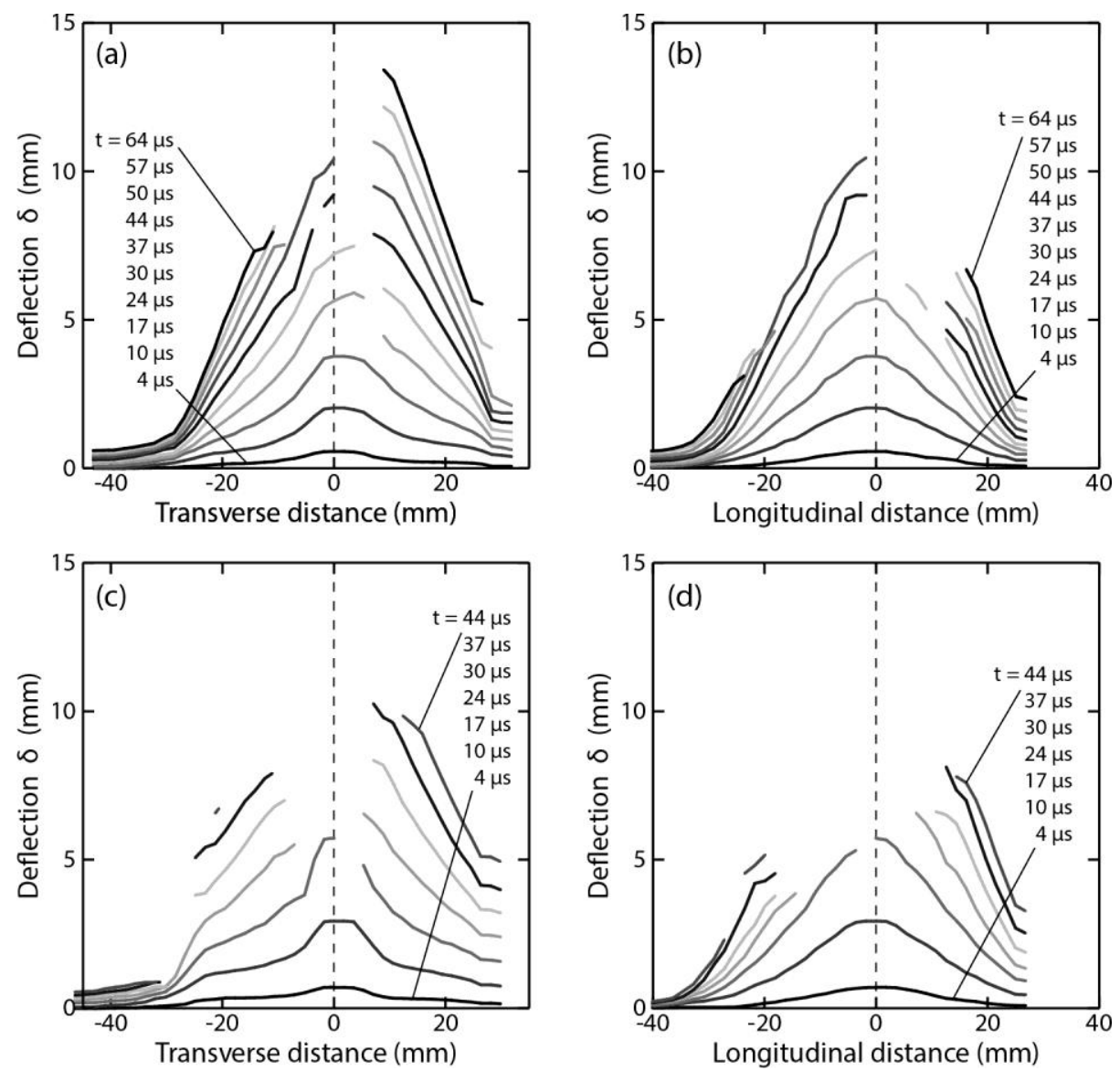

Figure 6.11. The out of plane deflection history along (a) transverse and (b) longitudinal profile coordinates, as identified in Figure 6.10(a), for the rear face cutout target prism base impacted at $V_{i}=1.71 \mathrm{~km} \mathrm{~s}^{-1}$. Analogous data for the sample impacted at $V_{i}=2.29 \mathrm{~km} \mathrm{~s}^{-1}$ is shown in (c) and (d).

The DIC determined velocity at the most deflected location, $\dot{\delta}_{\text {peak }}$, is plotted in Figure 6.12 as a function of time for the two impact velocities. For the $1.71 \mathrm{~km} \mathrm{~s}^{-1}$ impact, the rear face sheet accelerated to a maximum deflection rate $\dot{\delta}_{\max }=0.30 \mathrm{~km} \mathrm{~s}^{-1}$ at $t=17 \mu \mathrm{s}$. The $2.29 \mathrm{~km} \mathrm{~s}^{-1}$ impact required a similar time to reach $\dot{\delta}_{\max }=0.45 \mathrm{~km} \mathrm{~s}^{-1}$. In general, $\dot{\delta}_{\max }$ monotonically increased with impact velocity, Figure 6.13 , as did the terminal debris velocity (increasing from $V_{r}=0.43 \mathrm{~km} \mathrm{~s}^{-1}$ to $0.62 \mathrm{~km} \mathrm{~s}^{-1}$ ). While the values of $V_{r}$ were near or above the perforation limit of the laminate when impacted by an intact sphere, Table 6.3 , the difference between $\dot{\delta}_{\text {max }}$ and the faster traveling debris was 
only $0.1-0.2 \mathrm{~km} \mathrm{~s}^{-1}$ across the test velocity range. A laminate attached to the rear face sheet would therefore have been pre-accelerated by the motion of the large area face sheet bulge, which would have reduced the debris impact velocity in the laminate frame of reference.

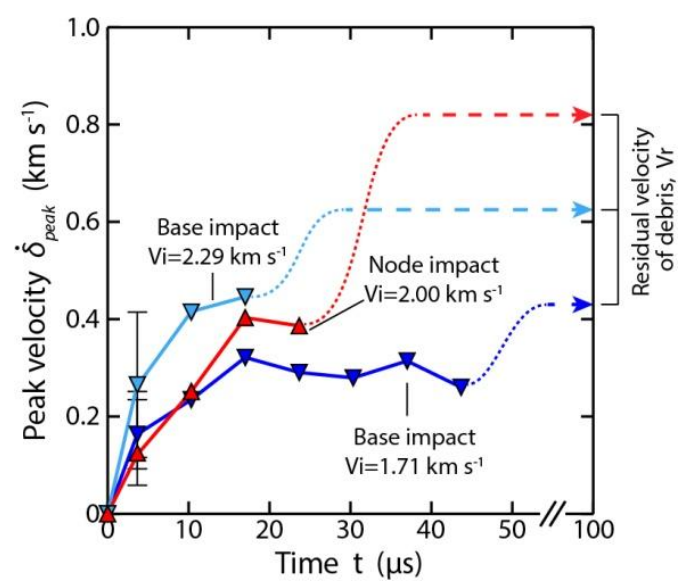

Figure 6.12. The peak out of plane velocity, $\dot{\delta}_{\text {peak }}$, versus time for rear face cutout targets. Two of the samples were impacted at a prism base and the third at a prism node. The terminal residual velocities after perforation are also shown.

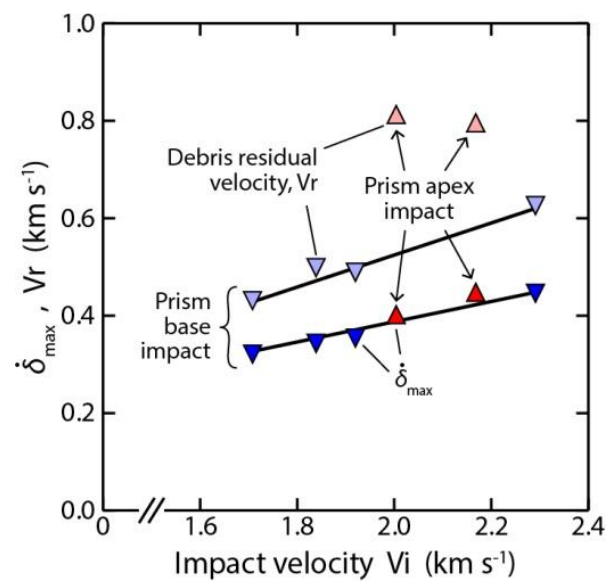

Figure 6.13. The debris residual velocity, $V_{r}$, and maximum out of plane velocity, $\dot{\delta}_{\text {max }}$, of rear face cutout targets versus impact velocity, $V_{i}$. Data for prism base impact is shown in dark $\left(\dot{\delta}_{\text {max }}\right)$ and light $\left(V_{r}\right)$ blue. Data for a prism apex impact is shown in red $\left(\dot{\delta}_{\text {max }}\right)$ and pink $\left(V_{r}\right)$. 


\subsubsection{Rear face cutout targets - ceramic prism apex impacts}

A high-speed video image sequence of the speckle coated rear face sheet of a rear face cutout target impact at a prism apex is shown in Figure 6.14. Two bulges were initially observed at $t=4 \mu \mathrm{s}$. The bulges occurred at the two corrugated web - face sheet nodes on either side of the base of the apex impacted ceramic prism. The peaks merged into one bulge with a $20 \mathrm{~mm}$ wide transverse front by $t=10 \mu \mathrm{s}$, Figure 6.15 . Two Yoriented cracks formed by $t=30 \mu$ s and allowed the portion of the rear face sheet under the impacted prism to be subsequently torn away, facilitating release of the debris, Figure 6.14. The out of plane velocity at the location of peak deflection, $\dot{\delta}_{\text {peak }}$, was shown as a function of time in Figure 6.12. The response was similar to those of the base impacts. However the residual velocity, $V_{r}=0.8 \mathrm{~km} \mathrm{~s}^{-1}$, of the debris in this case was substantially higher than the residual velocity for a prism base impact and about $0.4 \mathrm{~km} \mathrm{~s}^{-1}$ faster than the back face sheet maximum velocity prior to debris emission, Figure 6.13. Thus the rear face sheet of a sample impacted at a prism apex bulged at a similar out of plane velocity to a prism base impact, but the debris at face sheet failure travelled at more than twice the speed as that for a prism apex impact at similar incident velocity. 

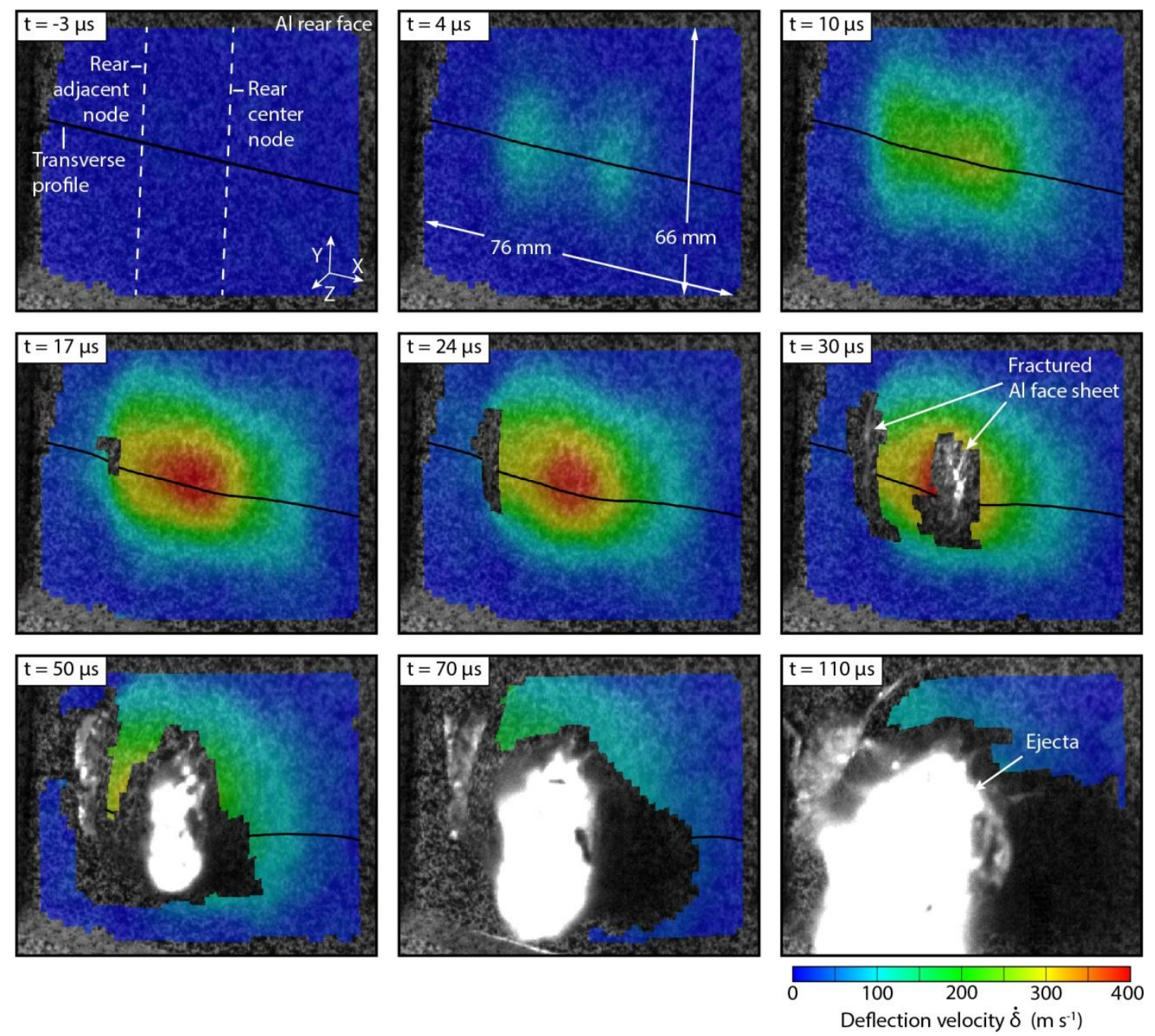

Figure 6.14. High-speed video image sequence of the aluminum surface of a rear face cutout target impacted at $V_{i}=2.00 \mathrm{~km} \mathrm{~s}^{-1}$ on a prism apex. A map of the DIC generated out of plane velocity, $\dot{\delta}$, component is superimposed. 


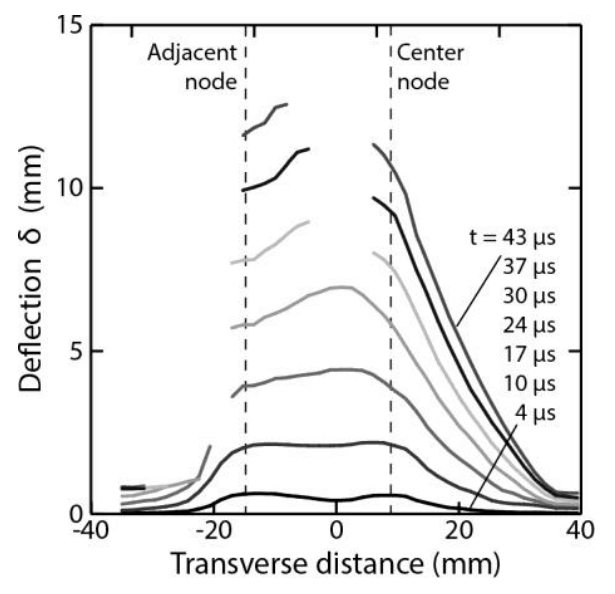

Figure 6.15. The temporal evolution of the deflection profile of a rear face cutout target impacted at a prism apex with a velocity of $V_{i}=2.00 \mathrm{~km} \mathrm{~s}^{-1}$.

\subsection{Discussion}

By impacting a model alumina filled hybrid sandwich panel at either a ceramic prism base or apex, it has been possible to modify the dynamic loading of a rear, $5.9 \mathrm{~mm}$ thick Dyneema ${ }^{\circledR}$ laminate. In Chapter 4, we show that the front (back supported) laminate of an encased aluminum plate impacted by the same, $12.7 \mathrm{~mm}$ diameter spherical steel projectile, was perforated once the impact velocity exceeded $0.2 \mathrm{~km} \mathrm{~s}^{-1}$, Table 6.3 . We therefore assume that the projectile velocity that penetrated the front laminate and struck the $1 \mathrm{~mm}$ thick front aluminum face sheet did so with an impact velocity reduced by $0.2 \mathrm{~km} \mathrm{~s}^{-1}$. This was always sufficient to perforate the aluminum face sheet and impact either the $22 \mathrm{~mm}$ wide base of the center ceramic prism or the apex of one of the adjacent prisms. The high hardness (14.1 GPa) and elastic stiffness (370 GPa) of the alumina resulted in sufficiently high contact pressures during impact to cause the projectile fragmentation at the prism interface. The low fracture toughness of the alumina (4-5 $\mathrm{MN} \mathrm{m}^{-3 / 2}$ ) resulted in the formation of a mescal zone of highly comminuted and pulverized ceramic under the projectile impact site. Cone and radial cracks readily form 
in alumina tiles [23], but these were disrupted here by the discontinuous nature of the prisms and the ductile aluminum webs. Nevertheless, as the impact velocity increased, the volume of damaged ceramic material increased. Since there was no ceramic disruption along the axis of the prisms, distance of damaged ceramic was greater along the corrugation direction (Y-axis) than transverse to it (X-axis).

The impulse applied to the rear laminate by the fragmented ceramic and projectile debris plume depended upon the site of impact. Impacts at a prism base resulted in a $50 \mathrm{~mm}$ wide square shaped region of the rear face sheet being displaced out of plane (Zdirection). After exceeding a displacement of $10 \mathrm{~mm}$, debris was ejected from the fractured rear aluminum face sheet as a more than $40 \mathrm{~mm}$ wide plume, with a velocity that increased from 0.43 to $0.62 \mathrm{~km} \mathrm{~s}^{-1}$ as the target incident velocity increased from 1.7 to $2.3 \mathrm{~km} \mathrm{~s}^{-1}$, Figure 6.13 . The greatly reduced debris velocity in comparison to the projectile incident velocity is attributed to the large volume (mass) of fragmented ceramic (approximately three prism cross sections), Figure 6.7, over which the projectile momentum was distributed, and the effectiveness, at lower impact velocities, of the front laminate at limiting reverse ejecta flow (and application of its reaction momentum). However, it is still remarkable that impacts at velocities as high as $2.7 \mathrm{~km} \mathrm{~s}^{-1}$ failed to rupture the rear laminate behind the location of debris impact.

A rationale for the response of the base impacted targets is shown schematically in Figure 6.16. The pressure applied by the debris was highest at the bottom (apex node) of the impacted cell causing the node and face sheet to fail in tension at the face sheet/node interface, Figure 6.16(a). The ductile aluminum face sheet then began to bend about plastic hinges that propagated to the left and right until arrest at the two adjacent 
nodes. Figure 6.6(b) and Figure 6.16(a) show that this accelerated a region of face sheet with a width given by $\sim 2 \mathrm{~L}_{\mathrm{c}}$ outwards reaching a maximum out of plane velocity that increased with impact velocity, Figure 6.13. This in turn accelerated the rear laminate with a force that was distributed over a similarly large area. Figure 6.12 shows that for the target impacted at $2.3 \mathrm{~km} \mathrm{~s}^{-1}$ the rear face sheet required approximately $10 \mu \mathrm{s}$ to reach a velocity of $0.45 \mathrm{~km} \mathrm{~s}^{-1}$. This was sufficient time for the $5.9 \mathrm{~mm}$ thick laminate (with a Z-direction longitudinal wave speed of slightly more than $2 \mathrm{~km} \mathrm{~s}^{-1}[10]$ and transverse hinge speed of about $0.3 \mathrm{~km} \mathrm{~s}^{-1}$ ) to transversely deflect, and maintain the impact pressure below the threshold needed to induce indirect tension mode of progressive ply failure. At higher target impact velocities, the faces of the fractured face sheet at the bottom apex node separated, and then released the debris plume to impact the inside of the rear laminate over a region with a width given of $\sim 1.5 \mathrm{~L}_{\mathrm{c}}$, Figure $6.16(\mathrm{~b})$. While the velocity of the debris over the test range could have been traveling in excess of $0.6 \mathrm{~km} \mathrm{~s}^{-1}$, the velocity difference between the debris and slower traveling aluminum face sheet was less than $0.2 \mathrm{~km} \mathrm{~s}^{-1}$, Figure 6.13. Since the Z-component motion of the face sheet provided a mechanism to reduce the velocity of the impacting debris in the laminate frame of reference, this limited the debris impact pressure, and ensured it not exceed the (typically 1.5 $\mathrm{GPa}$ ) pressure required to activate the indirect tension mode of failure. 

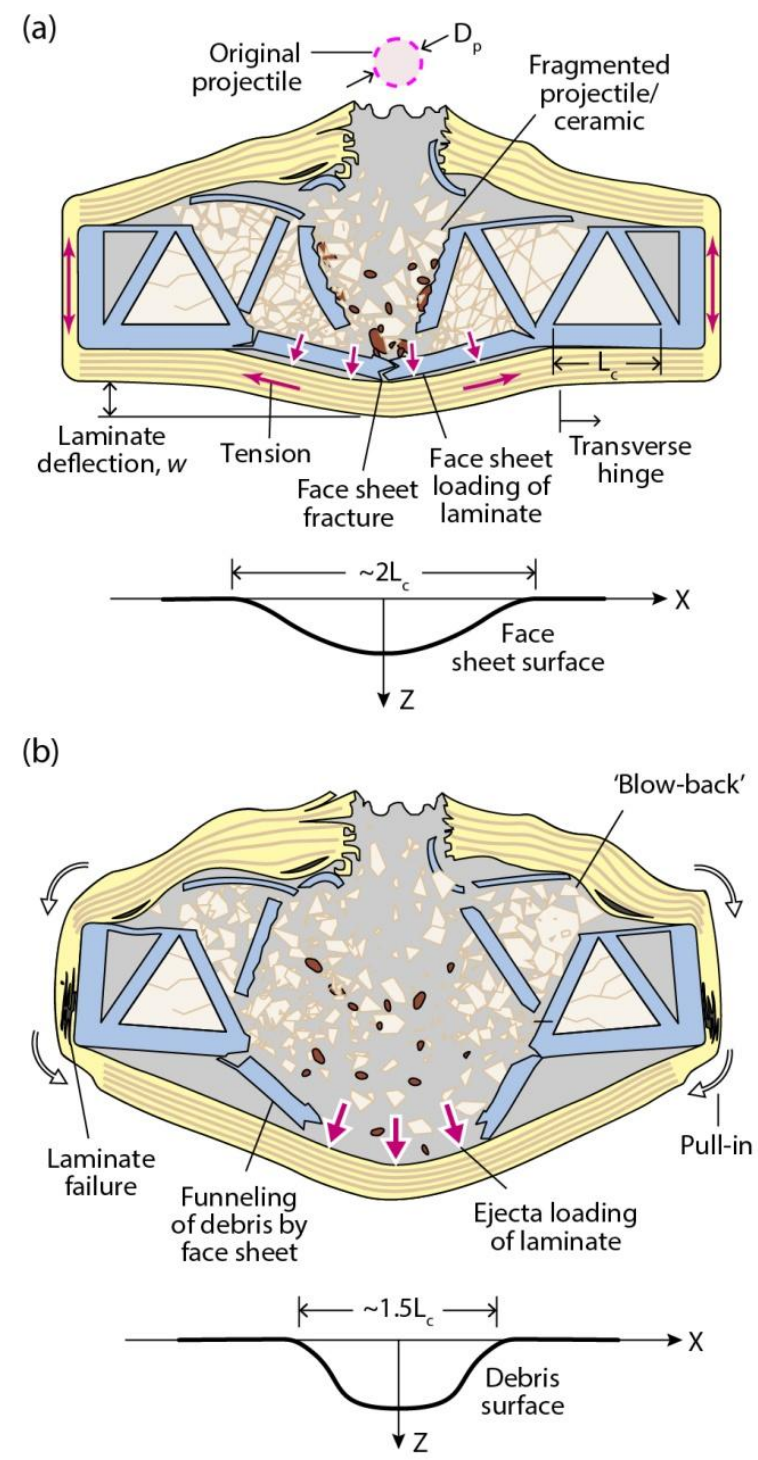

(c)

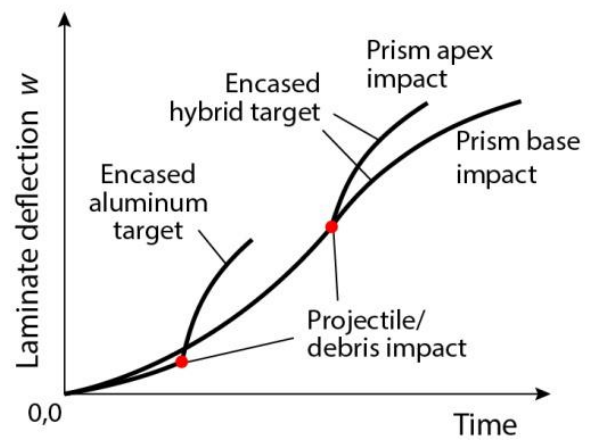

Figure 6.16. Schematic illustration showing the loading sequence of the rear laminate of the encased hybrid target impacted on a prism base. The rear laminate is (a) initially accelerated by the bulging rear face sheet and $(b)$ subsequently impacted by the released debris fragments. (c) Proposed defection history of the encased hybrid and encased aluminum targets both before and after impact by the debris/projectile. 
This observation is consistent with the behavior of the encased aluminum target, where an impact by the same spherical projectile caused the rear face of the aluminum plate to bulge (with an area limited to $\sim 2 \mathrm{x}$ the projectile diameter) prior to plate perforation, Figure 4.20. This resulted in a small pre-acceleration of the rear $5.9 \mathrm{~mm}$ thick HB26 laminate which was then impacted by an intact projectile that exited the rear of the aluminum plate with a velocity of $0.6 \mathrm{~km} \mathrm{~s}^{-1}$. This exceeded the ballistic limit of a $5.9 \mathrm{~mm}$ thick, stationary, edge clamped laminate impacted by this projectile, Table 6.3 . The indirect tension activation pressure (a property intrinsic to the laminate) remains the same in the pre-accelerated and stationary impact situations; only the impact velocity (and hence contact pressure) applied to the laminate in the pre-accelerated case was lower. Interestingly, impacts at a velocity of only $0.24 \mathrm{~km} \mathrm{~s}^{-1}$ are sufficient to start progressive indirect tensile failure, Table 6.3. The lack of any penetration in the encased hybrid target can be understood by considering the pressure applied to the laminate by a plume of debris particles scales as $\rho V_{i}^{2}$, where $\rho$ is the plume density. The steel projectile is many times denser than that of the plume of aluminum, alumina and fragmented debris. This, combined with the lower velocity of the debris plume and greater pre-acceleration applied to the laminate, resulted in $i$ ) an increase in penetration resistance of the rear laminate from $0.2 \mathrm{~km} \mathrm{~s}^{-1}$ to more than $0.6 \mathrm{~km} \mathrm{~s}^{-1}$, and ii) an increase in ballistic limit of the target from $1.4 \mathrm{~km} \mathrm{~s}^{-1}$ for the Dyneema ${ }^{\circledR}$ encased aluminum plate to more than $2.7 \mathrm{~km} \mathrm{~s}^{-1}$ for the prism base impacted samples studied here.

Debris penetration of the rear Dyneema ${ }^{\circledR}$ laminate after a prism apex impact can also be rationalized by a similar analysis. The impact again displaced the rear aluminum face sheet outward, at a velocity similar to that of a base prism impact, and therefore pre- 
accelerated the rear Dyneema ${ }^{\circledR}$ laminate to a similar Z-direction velocity, Figure 6.16(c). In an apex impact however, the mass of comminuted ceramic was significantly less, and the momentum transferred to it by the projectile therefore resulted in a higher debris velocity [17]. For a target impacted at $2 \mathrm{~km} \mathrm{~s}^{-1}$, the velocity difference between the debris and rear aluminum face sheet was approximately $0.4 \mathrm{~km} \mathrm{~s}^{-1}$ for a ceramic prism node but only $0.1 \mathrm{~km} \mathrm{~s}^{-1}$ for a base impact, Figure 6.13. It is expected that the debris impact velocity in the laminate frame of reference would have continued to increase with target impact velocity, and the higher pressure that was applied to the laminate resulted in perforation for the $2.5 \mathrm{~km} \mathrm{~s}^{-1}$ prism apex impact.

In cases where debris plume that exited the rear of the aluminum sandwich panel and was captured by the rear Dyneema ${ }^{\circledR}$ laminate, its kinetic energy could be dissipated by membrane stretching of the full thickness of the rear laminate. Pull-in from the side and front laminates, and straightening of excess consolidated Dyneema combined to a give larger Z-direction displacement than would have been possible if a side supported rear face laminate (i.e. a typical spall shield) had been impacted, and this presumably enabled kinetic energy dissipation at reduced the membrane stress. While no back laminate failure was observed for any of the prism base impacts, some tensile fracture at the sides of the samples impacted at the highest velocity was observed. This was not unexpected since the number of fibers aligned with the tensile load at the sides of the sample was only a half that of the back laminate. This mode of response, as well as deflection behavior, is similar to that observed when beams of the same laminate were impacted by low-density aluminum foam projectiles [13]. 
This study has identified mechanisms which when activated increase the ballistic resistance of a laminate. Placement of a suitable material between the projectile and laminate can allow temporal and spatial modification of the material that impacts a laminate at the rear of the target. The use of the hybrid core sandwich panel here provided an effective means of activating projectile defeat by controlling the area impacting the laminate backing. The study has not investigated the best hybrid sandwich structure design (e.g. face sheet thickness, web/face sheet nodal strength, cell topology and cell size), as well as material properties (e.g. density, yield strength, toughness and ductility), or the best ceramic type, dimensions, topology, etc. These would need to be considered in relation to the impactor (density, size, shape, velocity, obliquity of impact) as well as the type of laminate backing. The study has shown that laminate selection should address material indices that maximize both the usual membrane mode of action and the indirect tension mode of failure. It is finally noted that the laminate encasement method utilized here was of substantial benefit since it enabled the back, side and front portions of the laminate to all contribute to membrane stretching and eliminated stress concentrations at bolt hole attachments that would be otherwise necessary.

\subsection{Conclusions}

The impact performance of an UHMWPE fiber reinforced polymer matrix composite (HB26 grade Dyneema ${ }^{\circledR}$ ) has been studied using model test structures that enabled control of the spatial distribution and velocity of ejecta. The laminate encased an aluminum alloy sandwich panel whose hybrid corrugated cores contained alumina prisms. It has been found that: 
- $\quad$ Self-gripping, Dyneema ${ }^{\circledR}$ encased targets were not penetrated by impacts with a $12.7 \mathrm{~mm}$ diameter hardened chrome steel sphere at a velocity $V_{i} \geq 2.7 \mathrm{~km} \mathrm{~s}^{-1}$. This corresponded to a100\% increase in the ballistic limit compared to a target that had the same laminate encasement and areal density, but used a solid aluminum plate to interact with the projectile.

- Fragmenting, and spatially/temporally dispersing the momentum of the impact and pre-accelerating the rear Dyneema ${ }^{\circledR}$ laminate prior to debris impact combine to greatly increase the penetration resistance of the fiber-reinforced laminate.

- Distribution of the impact momentum by the hybrid core sandwich panel was controlled by ceramic fragmentation, locations of nodal failures and face sheet bending. Pre-acceleration of the rear laminate by bulging of the rear surface of the intervening panel reduced the debris impact velocity upon the rear laminate by at least $40 \%$. This reduced the incident pressure upon the laminate, and for prism base impacts was sufficient to avoid the indirect tension mechanism of failure.

- The kinetic energy of the debris plume exiting the hybrid core sandwich was dissipated by large deflections and membrane stretching of the full thickness laminate. The stress required for this has been reduced by engagement of Dyneema $^{\circledR}$ at the sides and front of the encasement. 


\subsection{References}

[1] O'Masta MR, Compton BG, Gamble EA, Zok FW, Deshpande VS, Wadley HNG. Ballistic impact response of an UHMWPE fiber reinforced laminate encasing an aluminum-alumina hybrid panel. Int J Impact Eng 2014:[Submitted for publication].

[2] Attwood JP, Khaderi S, Karthikeyan K, Fleck NA, O'Masta MR, Wadley HNG, et al. The out-of-plane compressive response of Dyneema composites. J Mech Phys Solids 2014:[Submitted for publication].

[3] Iremonger MJ. Polyethylene composites for protection against high velocity small arms bullets. In: Reinecke WG, editor. Proc. 18th Int. Symp. Ballist., San Antonio, TX: 1999, p. 946-54.

[4] Scott BR. The penetration of compliant laminates by compact projectiles. Proc. 18th Int. Symp. Ballist., San Antonio, TX: 1999, p. 1184-91.

[5] Zhang Z, Shen S, Huancheng S, Zhang D. Ballistic Penetration of Dyneema Fiber Laminate. J Mater Sci Technol 1998;14:265-8.

[6] Grujicic M, Arakere G, He T, Bell WC, Cheeseman BA, Yen C-F, et al. A ballistic material model for cross-plied unidirectional ultra-high molecular-weight polyethylene fiber-reinforced armor-grade composites. Mater Sci Eng A 2008;498:231-41.

[7] Greenhalgh ES, Bloodworth VM, Iannucci L, Pope D. Fractographic observations on Dyneema ${ }^{\circledR}$ composites under ballistic impact. Compos Part Appl Sci Manuf 2013;44:51-62.

[8] Karthikeyan K, Russell BP, Fleck NA, Wadley HNG, Deshpande VS. The effect of shear strength on the ballistic response of laminated composite plates. Eur J Mech ASolids 2013;42:35-53.

[9] Dehn JT. A unified theory of penetration. Aberdeen Proving Ground, MD: Ballistic Research Laboratory; 1986.

[10] Chapman DJ, Braithwaite CH, Proud WG. The Response of Dyneema to ShockLoading. AIP Conf Proc 2009;1195:1269-72.

[11] Hazell PJ, Appleby-Thomas GJ, Trinquant X, Chapman DJ. In-fiber shock propagation in Dyneema ${ }^{\circledR}$. J Appl Phys 2011;110:043504-1-043504-5.

[12] Cunniff PM. Dimensionless parameters for optimization of textile-based body armor systems. In: Reinecke WG, editor. Proc. 18th Int. Symp. Ballist., San Antonio, TX: Technomic Publishing Company, Inc; 1999, p. 1303-10.

[13] Karthikeyan K, Russell BP, Fleck NA, O'Masta MR, Wadley HNG, Deshpande VS. The soft impact response of composite laminate beams. Int J Impact Eng 2013;60:24-36.

[14] Yungwirth CJ, Radford DD, Aronson M, Wadley HNG. Experiment assessment of the ballistic response of composite pyramidal lattice truss structures. Compos Part B Eng 2008;39:556-69. 
[15] Yungwirth CJ, O’Connor J, Zakraysek A, Deshpande VS, Wadley HNG. Explorations of Hybrid Sandwich Panel Concepts for Projectile Impact Mitigation. J Am Ceram Soc 2011;94:s62-s75.

[16] Ni CY, Li YC, Xin FX, Jin F, Lu TJ. Ballistic resistance of hybrid-cored sandwich plates: Numerical and experimental assessment. Compos Part Appl Sci Manuf 2013;46:69-79.

[17] Wadley HNG, Dharmasena KP, O’Masta MR, Wetzel JJ. Impact response of aluminum corrugated core sandwich panels. Int J Impact Eng 2013;62:114-28.

[18] Wadley HNG, O'Masta MR, Dharmasena KP, Compton BG, Gamble EA, Zok FW. Effect of core topology on projectile penetration in hybrid aluminum/alumina sandwich structures. Int J Impact Eng 2013;62:99-113.

[19] O’Masta MR, Deshpande VS, Wadley HNG. Mechanisms of Projectile Penetration in Dyneema Encapsulated Aluminum Structures. Int J Impact Eng 2014; In Press.

[20] Schmidt TE, Tyson J, Galanulis K, Revilock DM, Melis ME. Full-field dynamic deformation and strain measurements using high-speed digital cameras. Proc SPIE, vol. 5580, Alexandria, VA: 2005, p. 174-85.

[21] Yu JH, Dehmer PG. Dynamic Impact Deformation Analysis Using High-speed Cameras and ARAMIS Photogrammetry Software. Aberdeen Proving Ground, MD: Army Research Laboratory; 2010.

[22] Hisley DM, Gurganus JC, Drysdale AW. Experimental Methodology Using Digital Image Correlation to Assess Ballistic Helmet Blunt Trauma. J Appl Mech 2011;78:051022-051022.

[23] Compton BG, Gamble EA, Zok FW. Failure initiation during impact of metal spheres onto ceramic targets. Int J Impact Eng 2013;55:11-23. 


\section{Chapter 7. Discussion}

\subsection{UHMWPE Composite Characterization}

The dissertation has characterized the structure and properties of six UHMWPE fiber reinforced $\left[0^{\circ} / 90^{\circ}\right]$ composites used for ballistic impact resistance applications and focused particular attention on the HB26 Dyneema ${ }^{\circledR}$ grade. This grade consists of gelspun Dyneema ${ }^{\circledR}$ grade SK76 UHMWPE fibers dispersed in a compliant polyurethane matrix, with an 83 wt.\% fiber fraction. The SK76 fiber has a highly oriented orthorhombic crystal structure, primarily in the chain extended conformation where the majority of the long molecular chain axis is aligned with the fiber axis. The $\mathrm{sp}^{3}$ bonded extended chain configuration leads to a fiber with a tensile strength of $3.6 \mathrm{GPa}$ and modulus of $110 \mathrm{GPa}$. The weak van der Waal bonds between the long molecules provide the fiber with bending flexibility but resulted in a material with extreme anisotropy.

The tensile response when the fiber is combined with the compliant matrix to form the $\left[0^{\circ} / 90^{\circ}\right] \mathrm{HB} 26$ laminate was approximately linear-elastic until its failure strength of $650 \mathrm{MPa}$ was approached. Fiber misalignment and waviness led to failure 
occurring in multiple discrete steps and reduced the tensile strength below rule of mixtures expectations, as has been also observed elsewhere [1-3]. It was shown in Section 3.2 that the laminate tensile response was dominated by the fiber reinforcement, with laminates using the same fiber but different matrices having similar responses, and those reinforced with stronger and stiffer fibers having stronger and stiffer laminate responses.

We then tested in interlaminar shear (Section 3.3) the HB26 laminate and measured a strength of only $1.8 \mathrm{MPa}\left(\sim 1 / 500^{\text {th }}\right.$ of the laminate tensile strength). This result is also consistent with measurements made elsewhere [2-4]. The interlaminar shear strength was determined to be a matrix controlled property, where laminates using polyurethane matrices (e.g. HB26) possess shear strengths of at least a factor of 3 greater than those of styrene based matrix laminates. A summary of the tensile and shear strength results is given in Table 2.2.

The interlaminar shear strength of the HB26 laminate was additionally tested as an out of plane (through thickness) compressive stress was applied to the gauge section. This pressure effect was investigated because a strong dependence of shear strength with transverse compression stress has been reported for bulk polymers [5], and is a key assumption in the indirect tension model of Attwood et al. [6]. The shear strength is shown in Section 3.4 to linearly increase with the applied pressure for pressures up to $13 \mathrm{MPa}$ (as assumed in the indirect tension model). Further assessment of the pressure dependence to the high compressive stresses ( 0.2 to $1 \mathrm{GPa})$ generated during impact is needed. 
The out of plane compression stress versus strain response of a unidirectional $\left[0^{\circ}\right]_{45} \mathrm{HB} 26$ laminate was presented in Section 3.5. The indirect tension model of Attwood et al. [6] is predicated on a highly anisotropic Poisson's ratio, in which a ply's lateral expansion is greater transverse to the fibers than parallel to the fibers during out of plane compressive loading. This anisotropy causes fibers in a $0^{\circ}$ ply to be loaded in tension due to the transverse expansion of the $90^{\circ}$ plies above and below. DIC analysis verified the Poisson's ratio was anisotropic, with a recorded Poisson ratio of near zero in the fiber direction, $v_{13}=0$, and $v_{23}=0.5$ in the transverse direction. Shear failure initiated at a stress of $\sim 20 \mathrm{MPa}$ and was followed by substantial plastic flow in the transverse direction. This is very different to the unstable failure observed in $\left[0^{\circ} / 90^{\circ}\right]$ laminates that failed when the tensile strength of the fibers was reached, and shows the importance of fiber architecture for activation of the indirect tension mechanism.

\subsection{Impact Response Mechanisms}

The dissertation also investigated the mechanisms that control penetration of UHMWPE fiber reinforced $\left[0^{\circ} / 90^{\circ}\right]$ composites during model ballistic impact events. For this purpose, targets consisting of an aluminum plate encased (wrapped) with a $\left[0^{\circ} / 90^{\circ}\right]$ Dyneema ${ }^{\circledR}$ grade HB26 laminate were impacted by $12.7 \mathrm{~mm}$ diameter, hardened steel, spherical projectiles at zero obliquity. The thickness of the aluminum plate was chosen so that impacts either above or below the plate/projectile shatter gap [7] could be utilized. This then allowed control of the degree of projectile fragmentation during penetration of the aluminum and impact with the rear Dyneema ${ }^{\circledR}$ laminate. A series of target designs allowed observation of the response of the laminate during three distinct loading 
conditions, Figure 7.1: (i) Impact by an intact projectile impacting a foundation supported laminate, (ii) impact by an intact projectile with an edge-clamped laminate, and (iii) impact by a fragmented projectile with an edge-clamped laminate.
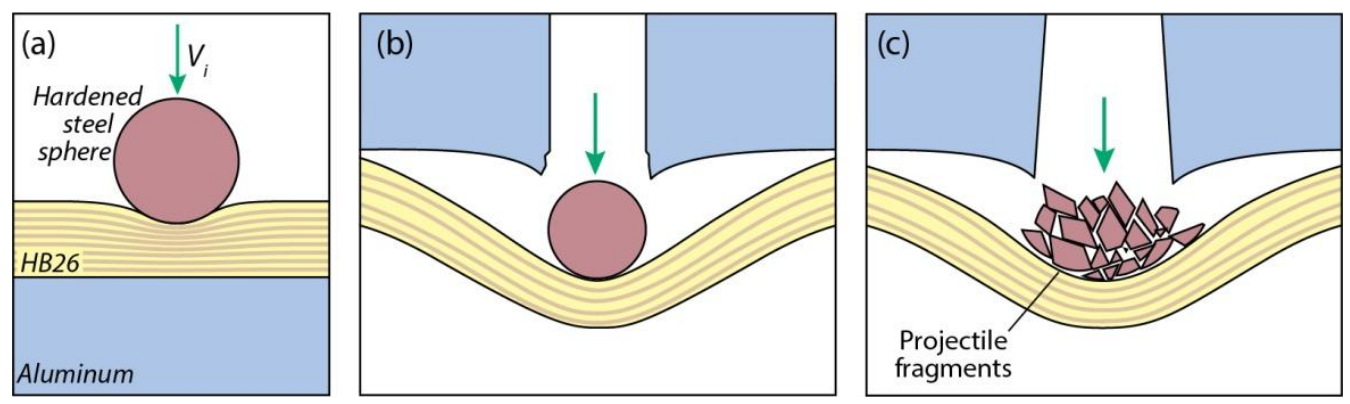

Figure 7.1. Three distinct loading scenarios investigated in the encased aluminum study used to elucidate penetration mechanisms. (a) An intact projectile impacting a foundation supported laminate. (b) An intact projectile impacting an edge-clamped laminate. (c) A fragmented projectile impacting an edge-clamped laminate.

When a laminate supported on a foundation was impacted, Figure 7.1(a), the laminate was penetrated by the intact projectile in a progressive manner, during which the depth of penetration increased with impact velocity. Chapter 4 clearly showed that the aluminum plate prevented the laminate from entering into a string-like, membrane stretching mode; the mechanism invoked by Phoenix and Porwal [8] to rationalize the dimensionless analysis of Cuniff [9]. Instead, the impact pressure applied by the projectile appeared to cause ply failure similar to the observations originally seen in references [10,11]. Woodward et al. [10] investigated the quasi-static and dynamic indentation of rigidly backed Kevlar and UHMWPE composite, and argued that indentation induced tensile stress in fibers just below the projectile was responsible for failure of fibers beneath the tip of the projectile. These papers did not provide a mechanism for the progressive penetration mode, but in a recently submitted paper with Attwood et al. [6], we show that the application of a compression stress to a $\left[0^{\circ} / 90^{\circ}\right]$ 
UHMWPE fiber reinforced laminate results in Poisson expansion of each ply transverse to its fiber direction. The transverse displacement results in shear lag loading of the adjacent ply's placing their fibers in tension. This provides a mechanism for conversion of an imposed compressive stress (like that under a projectile impact) to tension, and induces fiber tensile failure when the stress reaches the ply tensile strength. It is this indirect tension mechanism, due to the anisotropic Poisson expansion of the plies, that we argue is responsible for the penetration of these rear supported laminates.

When the laminate was allowed to deflect out of plane, in the edge clamped scenario, Figure 7.1(b), the ballistic limit (impact velocity for complete penetration of the laminate) was almost double that of the rear supported scenario. Again penetration occurred progressively, which was consistent with observations made elsewhere [3,1216]. However, upon comparing these results to those made by Karthikeyan et al. [3], who impacted the same edge clamped laminate with a similar projectile, we observed a smaller depth of penetration for a given impact velocity and a higher ballistic limit. The analysis in Chapters 4 and 6 shows that this unexpected result was determined to be a result of pre-acceleration of the Dyneema ${ }^{\circledR}$ laminate by the bulging rear face of the aluminum plate prior to its impact by the projectile, Figure 7.2(a). The out of plane displacement of the plate pushes upon the laminate, at a pressure insufficient to activate indirect tension fiber failure in the laminate, causing it to acquire an out of plane velocity component. As a result the contact pressure induced by the projectile, which depends upon the impact velocity in the laminate frame of reference, was reduced. An additional reduction in laminate depth of penetration (and increase in ballistic limit) was observed for edge-clamped laminates when the projectile was fragmented prior to laminate impact, 
Figure 7.2(c). In this case, we argue the laminate penetration resistance is enhanced by spatial and temporal dispersion of the impact impulse.

(a) Encased aluminum target

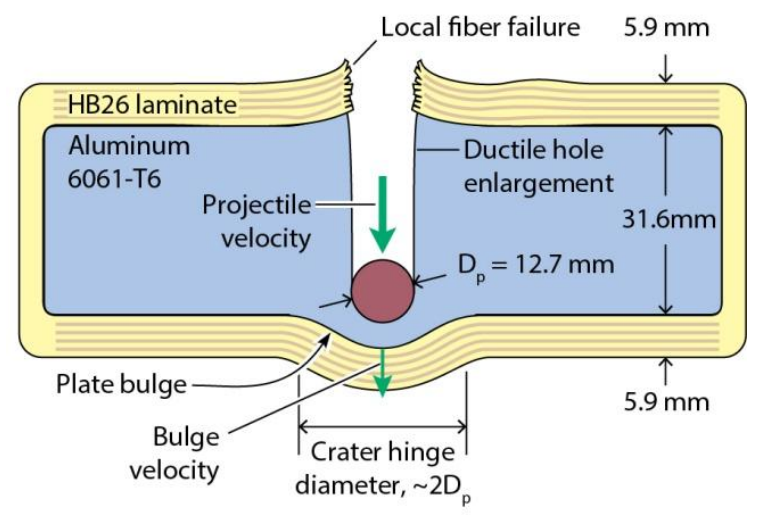

(b) Intact projectile impact

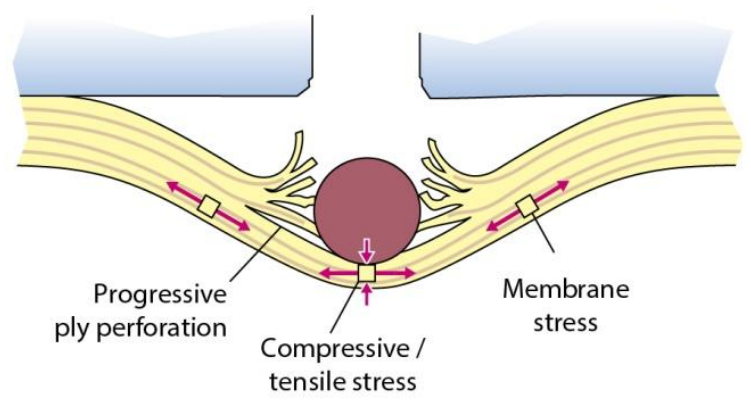

(c) Fragmented projectile impact

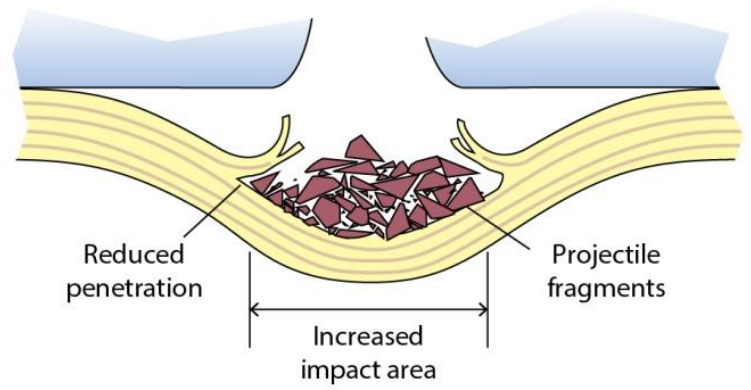

Figure 7.2. Schematic illustrations of an encased aluminum target after impact by a steel sphere. (a) Plate bulging prior to perforation pre-accelerates the edge-clamped rear laminate. The depth of penetration in the laminate upon impact by a projectile that penetrated the plate then depended upon whether the projectile (b) remained intact or (c) had fragmented.

It was not possible to directly observe the mechanism of penetration in the edge clamped experiments like that schematically illustrated in Figure 7.2(b). We can argue that the progressive penetration behavior is irreconcilable with the membrane stretching 
model as formulated in [8] which assumes all the ply's are similarly loaded and cannot therefore predict progressive penetration. Without a fully developed dynamic penetration model, it is difficult to conclusively prove penetration occurred by indirect tension. Although, preliminary results of a $2 \mathrm{D}$ impact experiment ${ }^{17}$, where a flat bottomed knifed edge impacts an edge clamped $\left[0^{\circ} / 90^{\circ}\right]$ composite beam, have captured high-speed footage of the progress failure of the composite. Furthermore, it is clear that test situations that reduced the pressure applied to the laminate by the projectile (such as unsupported rear face, pre-acceleration of the laminate, and distribution of fragmented projectile momentum over a large area) increased the impact performance of the laminate, and this is consistent with indirect tension penetration.

This paradox motivated the design of a Dyneema ${ }^{\circledR}$ HB26 laminate encased hybrid target in Chapter 6. In this model system, the high hardness of alumina ceramic prisms inserted into the triangular corrugation of a corrugated core sandwich panel were able to fragment an impacting projectile and distributed its impact momentum between the projectile fragments and ceramic particles over a very wide loading area. When such Dyneema ${ }^{\circledR}$ encased targets were impacted on a ceramic prism base, the interior surface of the rear Dyneema ${ }^{\circledR}$ laminate was loaded by a $\sim 50 \mathrm{~mm}$ wide square shaped region of debris with a velocity that increased with that of the projectile. However, even when the impact velocity was increased to $2,700 \mathrm{~m} \mathrm{~s}^{-1}$, and the debris impacted at more than $600 \mathrm{~m}$ $\mathrm{s}^{-1}$ (well above the laminate's ballistic limit of $450 \mathrm{~m} \mathrm{~s}^{-1}$ for the $12.7 \mathrm{~mm}$ diameter spherical projectile), the laminate was never penetrated.

\footnotetext{
${ }^{17}$ Performed by Vikram Deshpande's group at the University of Cambridge and shared by private communication.
} 
From these impact studies, a working, two mechanism, penetration hypothesis can be proposed. In an impact event, a region of compression develops in a $\left[0^{\circ} / 90^{\circ}\right]$ UHMWPE fiber reinforced laminate beneath the projectile. The magnitude of this stress scales with impact velocity, as well as the shape, density and sound speed of the projectile, Figure 7.3(b). If the impact induced compressive stress is sufficiently high, it is able to cause indirect tension and tensile fracture of a ply's fibers, Figure 7.3(c). The work done in failing the ply, thereby advancing the projectile a ply thickness, is supplied by a reduction of the kinetic energy of the projectile, and so as penetration continues, the projectile slows until the pressure it exerts on the laminate becomes insufficient to cause further indirect tension failure. The compressive stress exerted on the laminate by the projectile will also lessen with time if the laminate is allowed to transversely deflect, and thereby reduce the velocity difference between the laminate and projectile, Figure 7.3(c). Once below the critical pressure to activate indirect tension, if the intact remainder of the laminate is sufficiently thick, and if it is allowed to transversely deflect, the projectile is then brought to rest by out of plane panel displacement against the forces resisting membrane stretching, Figure 7.3(d). However if the residual intact laminate is insufficiently thick, the resultant membrane stresses can be sufficiently high, to activate a second, membrane mode stage of tensile fiber failure and laminate perforation. 


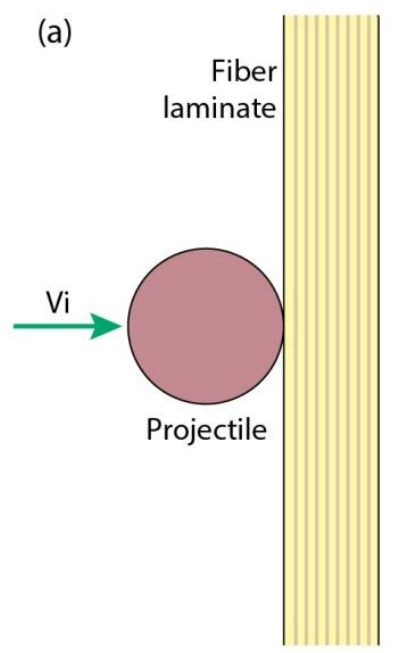

(c)

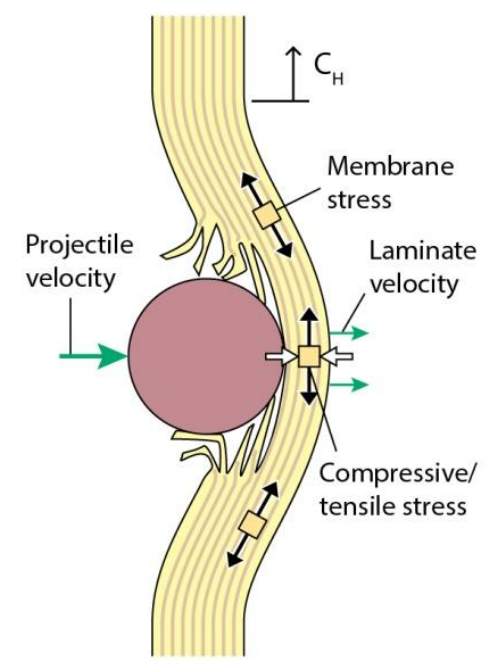

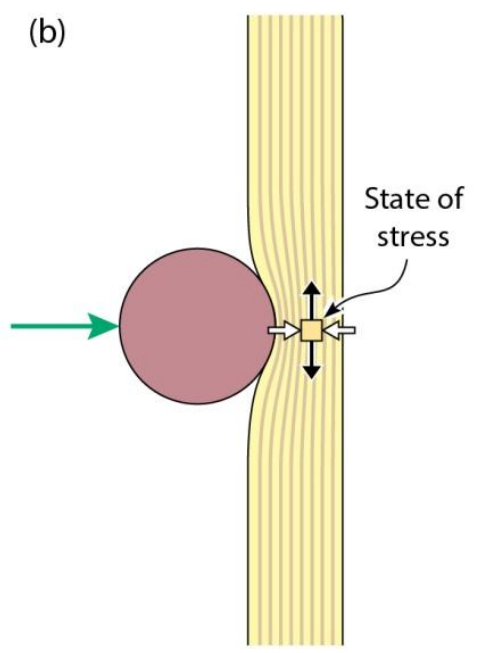

(d)

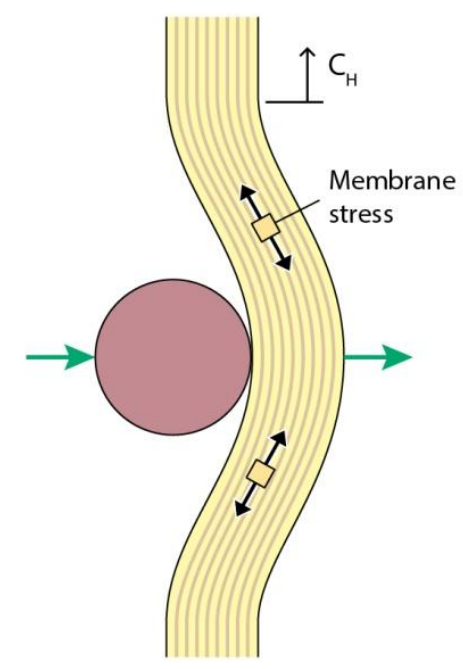

Figure 7.3. A proposed sequence of events for when (a) a spherical projectile impacts a stationary $\left[0^{\circ} / 90^{\circ}\right.$ ] polymer composite. (b) Inertial resistance prevents the laminate from immediately deflecting upon impact. The laminate underneath the projectile is placed in compression. This pressure is converted into a tensile stress in a ply's fiber direction by anisotropic expansion of adjacent $0^{\circ} / 90^{\circ}$ plies. (c) The velocity of the projectile is reduced by the work done in progressively failing the plies. If sufficient time has elapsed, the laminate may be simultaneously under membrane stretching and indirect tension. (d) If the laminate is sufficiently resistant to the penetration forces, it may enter into large deflections and resistant forces to stretching of the laminate will bring the projectile to rest.

The laminate encasement method utilized with these ballistic targets also enhanced the ballistic performance of the laminate once engaged in membrane stretching. This feature was particularly apparent in the encased hybrid test structure covered in Chapter 6 . The very high extensional wave speed $\left(\sim 10 \mathrm{~km} \mathrm{~s}^{-1}\right)$ enabled rapid 
communication of the membrane stretching force around the sides and to the front of the laminate. This enabled the displacement needed for out of plane deflection to be provided by extension of a laminate (gauge) length that was more than twice that of the sample width. In addition, extra displacement was provided by the small (but significant) excess

length of Dyneema ${ }^{\circledR}$ that was stored at the periphery of the sample. The combination of these two sources of material flow towards the site of debris loading would then reduce the deceleration, and associated forces of the laminate. It also resulted in increased alignment of the laminate's fibers with the debris loading axis which increased the efficiency with which the laminate could sustain the load. The overall effect was further delay in tensile failure and improved impact performance.

\subsection{Laminate Defect Effects}

Attwood et al. [6] showed that the out of plane (through thickness) uniform compression of a $\left[0^{\circ} / 90^{\circ}\right]$ polymer matrix UHMWPE fiber laminate is controlled by an indirect tension mechanism that creates a tensile stress in the fibers. When the tensile stress reaches the failure strength of the fibers, the sample catastrophically fails. Compressive strength results for six $\left[0^{\circ} / 90^{\circ}\right]$ UHMWPE reinforced laminates revealed a sample thickness effect unaddressed by Attwood et al. [6] . Thinner laminates had compressive strengths that were $30 \%$ to $40 \%$ below predictions, while the strength was restored by increasing the sample thickness. Detailed nondestructive characterization using micro-XCT methods, revealed the presence of both tunnel cracks and missing fiber defects. The former were very prominent, but were removed during the early stages of compression, and therefore had no effect upon the compressive strength. However, the 
missing fiber defects, which caused some regions of a laminate to fail before others (containing less fibers in the local load path), were shown to be responsible for the reductions in strength. Increasing the laminate thickness homogenized the defect distribution and improved the strength, but did not restore it to the level achievable if the defects had not been present.

Finally, the defects in laminates were traced to defects present in the pre-preg tape provided by the manufacture. Warm consolidating the pre-peg tapes into laminates at an elevated pressure did reduce the defect volume. However variability in ply thickness was also observed in the consolidated laminates and is believed to cause the observed variability in compressive strength. An effort by either the manufacture or the end user to reduce the missing fiber defects and ply variability would lead to significant improvements in the compressive strength.

\subsection{Suggestions for future work}

A summary of the average strength values measured for $H=1 \mathrm{~mm}$ thick samples of each material is shown in Figure 7.4. Also plotted is preliminary data for UHMWPE solid-state tapes. These tapes consist of ply's in which the UHMWPE is highly crystalline and highly oriented in one direction and have a similar thickness to those in the fiberreinforced laminates. These ply's are stacked in a $\left[0^{\circ} / 90^{\circ}\right]$ sequence and consolidated in the usual manner. These materials have $\sim 1 / 2$ the ply tensile strength of the strongest fiber reinforced laminates, X106 and X131, and approximately twice the shear strength of HB26. Unexpectedly, their compressive strength is equivalent or greater than all of the fiber-reinforced laminates. These materials appear to less efficiently convert compressive 
stress into tension and have a less prominent shear lag region, which allows them to sustain higher compressive stresses at a given length. This suggests that laminates reinforced with solid-state tapes would likely provide superior impact performance in the moments controlled by indirect tension to those reinforced with just fibers, even if the fiber laminates had modestly stronger tensile strengths than the solid-state tapes available today.

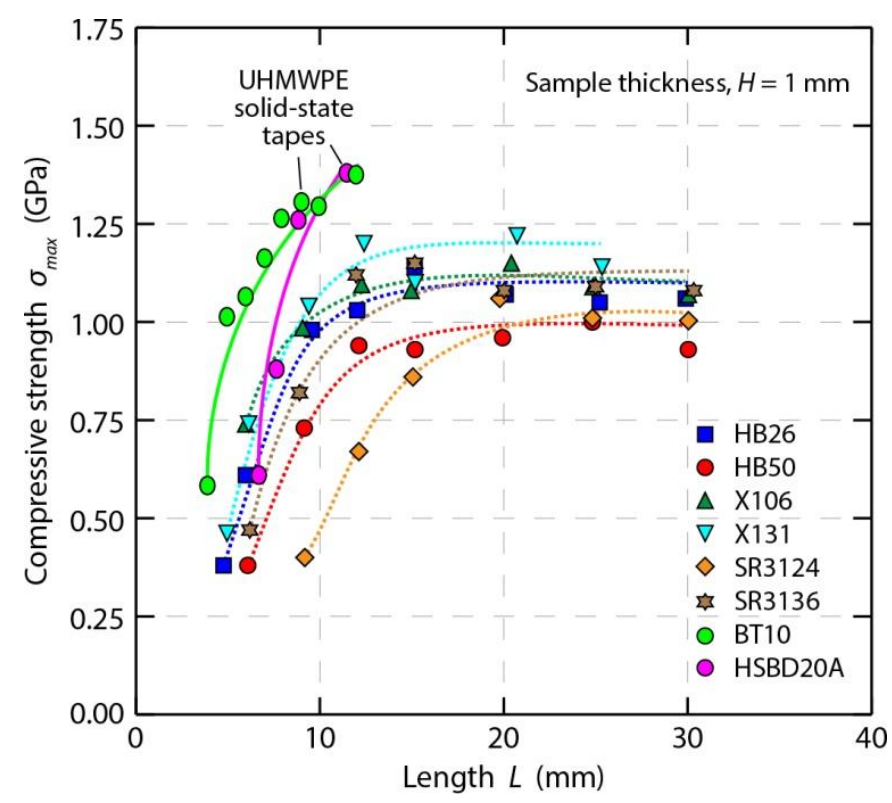

Figure 7.4. Average compression strength, $\sigma_{\max }$, plotted as a function of sample length, $L$, for all UHMWPE fiber laminate grades and two UHMWPE solid-state laminate grades, BT10 Dyneema $^{\circledR}$ and HSBD20A Tensylon ${ }^{\circledR}$ (DuPont, USA). BT10 data is from private communication with J.P. Attwood [17].

It also implies that a benefit may exist to a multi-grade composite lamination of solid-state tapes and fibers. Plies reinforced by solid-state tapes would be placed nearest the impact side, where the greatest resistance to indirect tension is needed to sustain the contact pressures by the projectile. Nearest the rear side of a laminate would be plies reinforced by the higher strength fibers that could sustain higher membrane stresses. The plies nearest the rear surface would also benefit from a fast transverse hinge velocity, 
facilitating out of plane deflection and precipitate the transition from a stage controlled by indirect tension to one controlled by membrane stretching, Figure 7.3(d). The optimal combination of material properties (tensile strength, shear strength, longitudinal wave speed, transverse wave speed, etc.) would then not only depend upon the expected impacting object (density, mass, velocity, etc.) but location within a laminate and the expected mechanism to be activated.

The following is a list of other possible ideas for continued work:

An improved understanding of quasi-static indentation is needed. This experiment presents a better approximation of the stress fields observed in a penetration event than the uniform compression experiment; although, the mathematic complexity of the problem increases.

Dynamic runs of quasi-static experiments should be tested. A potential envisioning would be a uniform compression or indentation test using a gas gun equipped with a split Hokinson pressure bar. This would be revealing of strain rate effects.

A continued effort into identifying the mechanisms present during impact of a polymer fiber reinforced composite is needed. A valuable experiment would be one that could clearly observe the penetration event and record the stresses, or strains, observed on either side of a laminate (even better if through the thickness) during penetration. Of particular interest are the 0.1-10 $\mu$ s after impact. However, projectile penetration of a laminate may be sufficiently complex to require a modeling effort to be able to completely capture the nuanced event. 
Ballistic impact tests on bi-layered laminations of solid-state reinforced plies backed by fiber reinforced plies could be performed. Documentation exists of an improved ballistic performance when layering plies reinforced with Tensylon ${ }^{\circledR}$ UHMWPE solid-state tape with plies reinforced with plies of Dyneema ${ }^{\circledR}$ UHMWPE fibers [18]. However, a more systematic study is needed to understand the source of the effect.

A systematic study of processing parameters on defect reduction could be useful. For instance, a study by Chiou et al. [19] has shown the consolidation pressure affects ultrasonic attenuation in the laminates. The appropriate consolidation parameters (temperature, pressure, vacuum, etc.) for minimizing defects within laminates assembled from various constitutive materials could be determined.

\subsection{References}

[1] Russell BP, Karthikeyan K, Deshpande VS, Fleck NA. The high strain rate response of Ultra High Molecular-weight Polyethylene: From fibre to laminate. Int J Impact Eng 2013;60:1-9.

[2] Karthikeyan K, Russell BP, Fleck NA, O’Masta MR, Wadley HNG, Deshpande VS. The soft impact response of composite laminate beams. Int J Impact Eng 2013;60:24-36.

[3] Karthikeyan K, Russell BP, Fleck NA, Wadley HNG, Deshpande VS. The effect of shear strength on the ballistic response of laminated composite plates. Eur J Mech ASolids 2013;42:35-53.

[4] Liu G, Thouless MD, Deshpande VS, Fleck NA. Collapse of a composite beam made from ultra high molecular-weight polyethylene fibres. J Mech Phys Solids 2014;63:320-35.

[5] Ward IM, Sweeney J. An Introduction to the Mechanical Properties of Solid Polymers. 2nd ed. Hoboken, NJ: John Wiley \& Sons, Inc.; 2004. 
[6] Attwood JP, Khaderi S, Karthikeyan K, Fleck NA, O’Masta MR, Wadley HNG, et al. The out-of-plane compressive response of Dyneema composites. J Mech Phys Solids 2014:[Submitted for publication].

[7] Dehn JT. A unified theory of penetration. Aberdeen Proving Ground, MD: Ballistic Research Laboratory; 1986.

[8] Phoenix SL, Porwal PK. A new membrane model for the ballistic impact response and V50 performance of multi-ply fibrous systems. Int J Solids Struct 2003;40:6723-65.

[9] Cunniff PM. Dimensionless parameters for optimization of textile-based body armor systems. In: Reinecke WG, editor. Proc. 18th Int. Symp. Ballist., San Antonio, TX: Technomic Publishing Company, Inc; 1999, p. 1303-10.

[10] Woodward RL, Egglestone GT, Baxter BJ, Challis K. Resistance to penetration and compression of fibre-reinforced composite materials. Compos Eng 1994;4:329-41.

[11] Scott BR, Cheeseman BA. The Mechanics of Projectile Arrest for Compliant Cross Plied Unidirectional Laminates. vol. Vol. 2, New Orleans, LA: 2008.

[12] Iremonger MJ. Polyethylene composites for protection against high velocity small arms bullets. In: Reinecke WG, editor. Proc. 18th Int. Symp. Ballist., San Antonio, TX: 1999, p. 946-54.

[13] Scott BR. The penetration of compliant laminates by compact projectiles. Proc. 18th Int. Symp. Ballist., San Antonio, TX: 1999, p. 1184-91.

[14] Zhang Z, Shen S, Huancheng S, Zhang D. Ballistic Penetration of Dyneema Fiber Laminate. J Mater Sci Technol 1998;14:265-8.

[15] Grujicic M, Arakere G, He T, Bell WC, Cheeseman BA, Yen C-F, et al. A ballistic material model for cross-plied unidirectional ultra-high molecular-weight polyethylene fiber-reinforced armor-grade composites. Mater Sci Eng A 2008;498:231-41.

[16] Greenhalgh ES, Bloodworth VM, Iannucci L, Pope D. Fractographic observations on Dyneema ${ }^{\circledR}$ composites under ballistic impact. Compos Part Appl Sci Manuf 2013;44:51-62.

[17] Attwood JP. BT10 tape model 2014.

[18] Lyons FS, Mears JA, Weedon GC, Owen L, Russell PA, Mitchell J, et al. Ballisticresistant article including one or more layers of cross-plies UHMWPE tape in combination with cross-plied fibers. US 7,972,679 B1, 2011.

[19] Chiou C-P, Margetan FJ, Barnard DJ, Hsu DK, Jensen T, Eisenmann D. Nondestructive characterization of UHMWPE armor materials. AIP Conf. Proc., vol. 1430, AIP Publishing; 2012, p. 1168-75. 


\section{Chapter 8. Conclusions}

The emergence of methods to make highly crystalline fibers from giant polyethylene molecules with molecular weights approaching $10^{7} \mathrm{Da}$ has resulted in the development of new fibrous materials with densities of $970 \mathrm{~kg} \mathrm{~m}^{-3}$, a tensile modulus approaching $200 \mathrm{GPa}$ and strengths in tension exceeding $3.5 \mathrm{GPa}$. This dissertation has investigated the structure, mechanical properties and mechanisms governing the penetration of polymer matrix composites reinforced with these UHMWPE fibers under impact loading scenarios. Emphasis was placed upon the identification and study of an indirect tension mode of penetration, where out of plane compressive loading of $\left[0^{\circ} / 90^{\circ}\right]$ laminates leads to loading of fiber in tension, and progressive projectile penetration of the laminate. The investigation was focused upon the Dyneema ${ }^{\circledR}$ grade HB26 system, but five other systems were used to investigate the generality of the findings. The five other $\left[0^{\circ} / 90^{\circ}\right]$ composite systems were assembled from matrices and UHMWPE fibers of differing strengths and produced by different manufactures. A summary of their measured properties, along with architecture and constituent material information, is provided in Table 2.2. HB26 consists of gel-spun Dyneema ${ }^{\circledR}$ grade SK76 UHMWPE 
fibers dispersed in a compliant polyurethane matrix, with an 83 wt.\% fiber fraction. This material was provided as a $\left[0^{\circ} / 90^{\circ}\right]_{2}$ preg tape, with a ply thickness of $\sim 67 \mu \mathrm{m}$. It was warm consolidated under confined compression to form $\left[0^{\circ} / 90^{\circ}\right]_{\mathrm{n}}$ laminates. The primary conclusions of the work can be grouped into those related to: $(i)$ material characterization, (ii) defect effects and (iii) impact resistance.

\section{Material characterization:}

- $\quad$ The $17 \mu \mathrm{m}$ diameter Dyneema ${ }^{\circledR}$ SK76 fiber was highly crystalline with $\sim 85$ vol. $\%$ in the orthorhombic structure with lattice parameters a $=7.43 \AA$, $\mathrm{b}=4.95 \AA$ and $\mathrm{c}=2.53 \AA$, while the remainder was amorphous [1]. The fibers were highly textured with the c-axis (molecular axis) is aligned along the fiber direction in an extended chain macro-conformation.

- The tensile response of the SK76 yarn (bundle of fibers) was linear-elastic, brittle and had a measured strength and modulus of $3.2 \mathrm{GPa}$ and $105 \mathrm{GPa}$. A $13 \mu \mathrm{m}$ diameter Dyneema ${ }^{\circledR} \mathrm{X} 131$ yarn had a measured yarn strength and modulus of $3.9 \mathrm{GPa}$ and $155 \mathrm{GPa}$, respectively. Both yarns were weaker than their reported fiber strengths, which was attributed to uneven loading of individual fibers in a yarn that contained wavy fibers.

- The tensile response of $\left[0^{\circ} / 90^{\circ}\right]$ laminates reinforced by UHMWPE fibers is dominated by the response of the reinforcing fiber. Waviness and misalignment of fibers within a laminate led to an uneven loading of individual fibers and a reduction of strength values below expectation.

- Using a double notch specimen, it was found that the interlaminar shear strength was matrix dominated; laminates with a polyurethane matrix had 
shear strengths of $\sim 1.5 \mathrm{MPa}\left(\sim 1 / 500^{\text {th }}\right.$ of the laminate's tensile strength) while the strength of styrene laminates was less than a $1 / 3^{\text {rd }}$ of this value.

- The shear strength of HB26 was shown to increase with out of plane (transverse) compression. This behavior has been incorporated into a model [2] of the out of plane compression behavior of $\left[0^{\circ} / 90^{\circ}\right]$ laminates controlled by an indirect tension mechanism.

- The Poisson expansion of a unidirectional $\left[0^{\circ}\right]_{45} \mathrm{HB} 26$ laminate under out of plane compression was shown to be anisotropic; another key assumption of the indirect tension model. The transverse Poisson's ratio is $v_{23}=0.5$, while the longitudinal Poisson's ratio is $v_{13}=0$. The unidirectional laminate failed in shear when the compressive stress reached $\sim 20 \mathrm{MPa}$ and was followed by significant plastic flow transverse to the fiber direction.

- $\quad$ Out of plane compression of six $\left[0^{\circ} / 90^{\circ}\right]$ cross ply UHMWPE reinforced laminates resulted in a catastrophic fracture, and at much higher compressive stresses $(>1 \mathrm{GPa})$ than the unidirectional HB26 laminate. These experiments indicated that the out of plane compressive strength of $\left[0^{\circ} / 90^{\circ}\right]$ laminates increased as: the ply thickness decreased, the ply strength increased, the laminate shear strength increased, and the coefficient of laminate shear hardening with compressive stress increased. All of these trends are consistent with indirect tension model predictions.

- A previously un-predicted sample thickness effect was observed. Thinner laminates had compressive strengths that were 30 to $40 \%$ below indirect tension model predictions. However, the strength was restored by increasing 
laminate thickness. As sample thickness increased, the out of plane compressive strength was found to approach that predicted by a recently proposed indirect tension model [2].

\section{Defect effects:}

- A combination of optical and ultrasonic C-scan imaging in conjunction with microXCT was used to identify two classes of defects in UHMWPE $\left[0^{\circ} / 90^{\circ}\right]$ polymeric composites; namely $i$ ) elongated void-like defects consisting of pockets of missing fibers and resin that spanned long distances $(>150 \mathrm{~mm})$ in the fiber direction of a ply, and ii) tunnel cracks arising from anisotropic thermal strains during the cooling after the consolidation process.

- The tunnel cracks are shown to be consistent with the negative coefficient of thermal expansion in the ( $\mathrm{sp}^{3}$ bonded) fiber direction and the large positive CTE in (van der Waals bonded) directions transverse to the fibers.

- Removal of tunnel cracks, by cold pressing the laminate, has no effect upon the transverse compressive strength of the laminate.

- The missing fiber defects are traced to defects present in the pre-preg tape. Warm consolidating the pre-peg tapes into laminates at an elevated pressure healed some of the defects and reduced the defect volume.

- A simple statistical model, that assumed defects to be randomly distributed and independent, successfully simulated optical images of bands of missing fiber defects stacked in the thickness direction of laminates.

- Using pressure sensitive film, regions containing stacks of missing fiber defects were shown to support a reduced compressive load. This uneven 
pressure distribution resulted in some regions of a laminate reaching the critical stress needed to activate indirect tensile failure before other regions. Thus, missing fiber defects degrade the out of plane compressive strength of a laminate.

- The reduction in strength due to these defects was captured by modeling of the load-shielding mechanism that incorporated the statistical prevalence of the defects. The model also captured the recovery of strength with increased sample thickness, and showed it to be due to homogenization of the defect distribution.

- A bimodal ply thickness test revealed that the compressive response was governed by the thickest ply (which fails at a lower strengths). Therefore, the ply thickness variability in these laminates is believed to cause the observed variability in compressive strength. This test also revealed the potential for strength degradation if thicker plies are introduced in a lay-up.

\section{Impact resistance:}

- Penetration of laminates either supported on a foundation or edge restrained occurs by the progressive fracture of fiber plies under the impactor, with the number of fractured plies increasing with impact velocity. The ballistic resistance of a laminate supported on a foundation is significantly lower (by nearly a factor of two) compared to that of edge restrained plates.

- The mechanism of penetration of laminates supported on a foundation by a blunt nose projectile or indenter was compression induced tensile fiber failure, 
identical to the indirect tension mechanism activated under uniform compression of a $\left[0^{\circ} / 90^{\circ}\right]$ architecture laminate.

- $\quad$ Since the compressive stress scales with the square of the impact speed, quite low velocity impacts can initiate failure of near surface plies. However, substantial dissipation of kinetic energy occurs during the penetration of a ply, and projectiles can be rapidly reduced in velocity until the pressure they apply is insufficient to cause rupture.

- In edge supported laminates, the residual kinetic energy at the cessation of indirect tension failure is dissipated by membrane stretching mode.

- If laminates are pre-accelerated, by low contact pressure inducing mechanisms, an initial velocity is imparted to the laminate prior to impact by a projectile, and the "apparent" ballistic limit of the laminate is enhanced. This results from a lowering of the projectile velocity in the laminate frame of reference (and thus reduction of the applied pressure) for of given projectile velocity in the laboratory reference frame.

- Fragmentation and concomitant spatial and temporal dispersion of the projectile by an intervening medium increases the ballistic limit of the laminate by distributing the interfacial forces between the projectile and laminate over a larger area, thereby reducing the contact pressure.

- Panels that are placed between the projectile and laminate that can both preaccelerate the laminate and spatially/temporally disperse the momentum of the impact are shown to greatly enhance the penetration resistance of the laminate. 
- If penetration is not activated, the kinetic energy of the impact is then dissipated by large deflections and membrane stretching of the full thickness the laminate. Failure will then occur where the stress is highest, which is often at a source of stress concentration such as the grips.

\subsection{References}

[1] O’Masta MR. Ultra-high molecular-weight polyethylene composites: structure, properties and ballistic response. Master's Thesis. University of Virginia, 2010.

[2] Attwood JP, Khaderi S, Karthikeyan K, Fleck NA, O’Masta MR, Wadley HNG, et al. The out-of-plane compressive response of Dyneema composites. J Mech Phys Solids 2014:[Submitted for publication]. 


\section{Appendix A. Tensile properties of high- performance fibers}

The data used to generate the material maps in Figure 1.3 is listed in Table A1 along with the details of the sources of the information. 
Table A-4. The data for the mechanical properties high performance fibers used to generate Fig. 1. The sources of information are included in the table for each case.

\begin{tabular}{|c|c|c|c|c|c|c|c|}
\hline Fiber & $\begin{array}{c}\rho \\
\left(\mathrm{Mg} \mathrm{m}^{-3}\right) \\
\end{array}$ & $\begin{array}{c}E \\
(\mathrm{GPa}) \\
\end{array}$ & $\begin{array}{c}E / \rho \\
\left(\mathrm{kJ} \mathrm{g}^{-1}\right) \\
\end{array}$ & $\begin{array}{c}\sigma_{f} \\
(\mathrm{GPa})\end{array}$ & $\begin{array}{c}\sigma_{f} / \rho \\
\left(\mathrm{kJ} \mathrm{g}^{-1}\right) \\
\end{array}$ & $\begin{array}{c}\varepsilon_{f} \\
(\%)\end{array}$ & $\begin{array}{c}c^{*} \\
\left(\mathrm{~m} \mathrm{~s}^{-1}\right) \\
\end{array}$ \\
\hline \multicolumn{8}{|l|}{ Alumina } \\
\hline Nextel 312 [1] & 2.7 & 150 & 56 & 1.7 & 0.6 & 1.1 & 298 \\
\hline Nextel 440 [1] & 3.05 & 190 & 62 & 2.0 & 0.7 & 1.1 & 301 \\
\hline Nextel 610 [1] & 3.90 & 380 & 97 & 3.1 & 0.8 & 0.8 & 317 \\
\hline Nextel 720 [1] & 3.4 & 260 & 76 & 2.1 & 0.6 & 0.8 & 279 \\
\hline \multicolumn{8}{|l|}{ Aramid } \\
\hline Kevlar $29[2,3]$ & 1.44 & $70-91$ & $49-63$ & $2.9-3.0$ & $2.0-2.1$ & $3.0-4.2$ & $621-671$ \\
\hline Kevlar $49[2,3]$ & 1.44 & $113-120$ & $78-83$ & 3.0 & 2.1 & $1.2-2.6$ & $487-619$ \\
\hline Kevlar $129[2,3]$ & 1.44 & $96-99$ & $67-69$ & $2.9-3.4$ & $2.0-2.4$ & $3.3-3.5$ & $648-695$ \\
\hline Kevlar 149 [4] & 1.47 & 185 & 126 & 3.4 & 2.3 & 2.0 & 638 \\
\hline Kevlar KM2 [2,3,5] & 1.44 & $70-85$ & $49-59$ & $3.3-3.9$ & $2.3-2.7$ & $3.8-4.5$ & $681-776$ \\
\hline Basalt [6] & $2.56-2.66$ & $53-62$ & $20-24$ & $1.6-2.0$ & $0.6-0.8$ & $2.7-3.6$ & $336-400$ \\
\hline \multicolumn{8}{|l|}{ Boron Carbide } \\
\hline 4-mil [7] & 2.54 & 400 & 157 & 3.6 & 1.4 & 0.9 & 431 \\
\hline 5.6-mil [7] & 2.38 & 400 & 168 & 4.0 & 1.7 & 1.0 & 478 \\
\hline \multicolumn{8}{|l|}{ Carbon } \\
\hline Hexcel AS4 [8] & 1.79 & 231 & 129 & 4.4 & 2.5 & 1.8 & 643 \\
\hline Hexcel IM7 [9] & 1.78 & 276 & 155 & 5.6 & 3.1 & 1.8 & 733 \\
\hline Hexcel IM9 [10] & 1.80 & 304 & 169 & 6.1 & 3.4 & 1.9 & 765 \\
\hline Toray M35J [11] & 1.75 & 343 & 196 & 4.7 & 2.7 & 1.4 & 636 \\
\hline Toray M45J [12] & 1.84 & 436 & 237 & 4.2 & 2.3 & 1.0 & 554 \\
\hline Toray M60J [13] & 1.93 & 588 & 305 & 3.9 & 2.0 & 0.7 & 491 \\
\hline Toray T300 [14] & 1.76 & 230 & 131 & 3.5 & 2.0 & 1.5 & 560 \\
\hline Toray T700S [15] & 1.80 & 230 & 128 & 4.9 & 2.7 & 2.1 & 689 \\
\hline Toray T800S [16] & 1.80 & 294 & 163 & 5.9 & 3.3 & 2.0 & 747 \\
\hline Toray T1000G [17] & 1.80 & 294 & 163 & 6.4 & 3.5 & 2.2 & 788 \\
\hline
\end{tabular}




\begin{tabular}{|c|c|c|c|c|c|c|c|}
\hline Fiber & $\begin{array}{c}\rho \\
\left(\mathrm{Mg} \mathrm{m}^{-3}\right) \\
\end{array}$ & $\begin{array}{c}E \\
(\mathrm{GPa}) \\
\end{array}$ & $\begin{array}{c}E / \rho \\
\left(\mathrm{kJ} \mathrm{g}^{-1}\right) \\
\end{array}$ & $\begin{array}{c}\sigma_{f} \\
(\mathrm{GPa})\end{array}$ & $\begin{array}{c}\sigma_{f} / \rho \\
\left(\mathrm{kJ} \mathrm{g}^{-1}\right)\end{array}$ & $\begin{array}{c}\varepsilon_{f} \\
(\%)\end{array}$ & $\begin{array}{c}c^{*} \\
\left(\mathrm{~m} \mathrm{~s}^{-1}\right) \\
\end{array}$ \\
\hline \multicolumn{8}{|l|}{ Glass } \\
\hline $\mathrm{E}[3,18,19]$ & $2.54-2.58$ & $69-74$ & $27-29$ & $3.4-3.8$ & $1.3-1.5$ & $4-4.8$ & $525-570$ \\
\hline $\mathrm{S} 2[18]$ & 2.49 & 87 & 35 & 4.8 & 1.9 & 5.7 & 689 \\
\hline \multicolumn{8}{|l|}{ LPC polyester } \\
\hline Vectran HS [20] & 1.4 & 80 & 57 & 2.5 & 1.8 & 2.7 & 570 \\
\hline Vectran HT [21] & 1.4 & 75 & 54 & 3.2 & 2.1 & 4.3 & 686 \\
\hline Vectran M [20] & 1.4 & 79 & 56 & 1.2 & 0.8 & 1.7 & 374 \\
\hline Vectran NT [21] & 1.4 & 52 & 37 & 1.1 & 0.7 & 2.1 & 357 \\
\hline Vectran UM [21] & 1.4 & 103 & 74 & 3.0 & 1.8 & 2.9 & 604 \\
\hline \multicolumn{8}{|l|}{$\mathrm{PBO}$} \\
\hline Zylon AS $[3,4]$ & 1.54 & 180 & 117 & 5.8 & 3.5 & 3.5 & 893 \\
\hline Zylon HM [3,4] & 1.56 & 270 & 173 & 5.8 & 2.5 & 2.5 & 849 \\
\hline $\begin{array}{l}\text { PIPD } \\
\quad \text { M5 }[2,4]\end{array}$ & 1.70 & $300-330$ & $176-194$ & $3.5-8.5$ & $2.1-5.0$ & $2.5-4.5$ & $864-940$ \\
\hline \multicolumn{7}{|l|}{ Polyamide } & $480-690$ \\
\hline \multicolumn{7}{|l|}{ Polypropylene } & 431 \\
\hline \multicolumn{8}{|l|}{ Silicon Carbide } \\
\hline Nicalon NL-200 [23] & 2.55 & 220 & 86 & 3.0 & 1.2 & 1.4 & 421 \\
\hline SCS-6 [24] & 3.08 & 380 & 123 & 3.9 & 1.3 & 1.0 & 416 \\
\hline SCS-9A [24] & 2.8 & 307 & 110 & 3.6 & 1.3 & 1.2 & 429 \\
\hline SCS-Ultra [24] & 3.08 & 415 & 135 & 5.9 & 1.9 & 1.4 & 541 \\
\hline \multicolumn{8}{|l|}{ UHMWPE } \\
\hline Dyneema $^{\circledR}$ SK60 [4] & 0.97 & 89 & 92 & 2.7 & 2.8 & 3.5 & 776 \\
\hline Dyneema $^{\circledR}$ SK65 [4] & 0.97 & 95 & 98 & 3.0 & 3.1 & 3.6 & 820 \\
\hline Dyneema ${ }^{\circledR}$ SK75 [4] & 0.97 & 107 & 110 & 3.4 & 3.5 & 3.8 & 888 \\
\hline Dyneema $^{\circledR}$ SK76 [4] & 0.97 & 116 & 120 & 3.6 & 3.7 & 3.8 & 917 \\
\hline Spectra $900[3,25]$ & 0.97 & $73-79$ & $75-81$ & $2.3-2.6$ & $2.4-2.7$ & $2.8-3.9$ & $670-758$ \\
\hline Spectra $1000[2,3,26]$ & 0.97 & 97- 120 & $100-124$ & $2.6-3.3$ & $2.7-3.4$ & $2.8-3.5$ & $749-807$ \\
\hline Spectra $2000[3,27]$ & 0.97 & $116-124$ & $120-128$ & $3.2-3.3$ & $3.3-3.4$ & $2.9-3.0$ & $810-836$ \\
\hline Spectra 3000 [28] & 0.97 & $115-122$ & $118-126$ & $3.2-3.4$ & $3.4-3.5$ & 3.3 & $844-867$ \\
\hline
\end{tabular}




\section{References}

[1] 3M Nextel. 3M Nextel Ceramic Fiber Typical Properties 2012.

[2] Cunniff PM, Auerbach MA, Vetter E, Sikkema DJ. High performance "M5" fiber for ballistics/structural composites. Proc. 23rd Army Sci. Conf., Orlando, FL: 2002.

[3] Lane R. High Performance Fibers for Personnel and Vehicle Armor Systems: Putting a Stop to Current and Future Threats. AMPTIAC Q 2005;9:1-10.

[4] Afshari M, Sikkema DJ, Lee K, Bogle M. High Performance Fibers Based on Rigid and Flexible Polymers. Polym Rev 2008;48:230-74.

[5] Cheng M, Chen W, Weerasooriya T. Mechanical Properties of Kevlar KM2 Single Fiber. J Eng Mater Technol 2005;127:197-203.

[6] Deák T, Czigány T. Chemical Composition and Mechanical Properties of Basalt and Glass Fibers: A Comparison. Text Res J 2009;79:645-51.

[7] Specialty Materials, Inc. Boron Fiber Properties n.d.

[8] Hexcel Corp. HexTow AS4 Carbon Fiber: Product Data 2010.

[9] Hexcel Corp. HexTow IM7 Carbon Fiber: Product Data 2010.

[10] Hexcel Corp. HexTow IM9 Carbon Fiber: Product Data 2010.

[11] Toray Carbon Fibers America, Inc. M35J Data Sheet. n.d.

[12] Toray Carbon Fibers America, Inc. M46J Data Sheet. n.d.

[13] Toray Carbon Fibers America, Inc. M60J Data Sheet. n.d.

[14] Toray Carbon Fibers America, Inc. T300 Data Sheet. n.d.

[15] Toray Carbon Fibers America, Inc. T700S Data Sheet. n.d.

[16] Toray Carbon Fibers America, Inc. T800S Data Sheet. n.d.

[17] Toray Carbon Fibers America, Inc. T1000G Data Sheet. n.d.

[18] Gibson RF. Principles of composite material mechanics. 2nd ed. Boca Raton: CRC Press; 2007.

[19] Owens-Corning. OCV Reinforcements Properties Summary 2012.

[20] Pegoretti A, Zanolli A, Migliaresi C. Preparation and tensile mechanical properties of unidirectional liquid crystalline single-polymer composites. Compos Sci Technol 2006;66:1970-9.

[21] Vectran Fiber, Inc. Vectran Fiber Strength Tensile Properties n.d.

[22] Bolduc M, Lazaris A. Spider Silk-Based Advanced Performance Fiber for Improved Personnel Ballistic Protection Systems. Defence R\&D Canada Valcartier; 2002.

[23] Ishikawa T. Recent developments of the SiC fiber Nicalon and its composites, including properties of the $\mathrm{SiC}$ fiber Hi-Nicalon for ultra-high temperature. Compos Sci Technol 1994;51:135-44.

[24] Specialty Materials, Inc. Silicon Carbide Fiber Properties n.d.

[25] Honeywell International Inc. Honeywell Spectra fiber 900: Product Information Sheet 2010. 
[26] Honeywell International Inc. Honeywell Spectra fiber 1000: Product Information Sheet 2010.

[27] Honeywell International Inc. Honeywell Spectra fiber 2000: Product Information Sheet 2010.

[28] Honeywell International Inc. Honeywell Spectra fiber 3000: Product Information Sheet 2013. 


\section{Appendix B. Measurement length changes from teh high speed images}

The pull-in, $\ell$, and stretched length, $L_{i}$, of the Dyneema ${ }^{\circledR}$ rear face are measured from the oblique and profile high speed images, such as those in Figure 4.12. The profile images are directly used to measure the arc-length, $L_{i}$, between the fiducial markers using the "string length" function within the Phantom camera software. However, measurement of the pull-in, $\ell$, from the oblique images requires a parallax correction to be performed using the image processing toolbox in the software MATLAB as follows.

Consider the square grid as shown in Figure B.1. When this square grid is observed at an angle a distorted image is obtained on the plane of view. We use the projective transformation in the "maketform" function in MATLAB to construct the transformation by providing the actual and observed 2D co-ordinates of the four corners of one of the grid squares. This transformation is then applied to the oblique images using the "imtransform" function in MATLAB so as to correct the image for the parallax. The 
measurements of $\ell$ are made by counting pixels to measure the movement of the fiducial markers between consecutive transformed high-speed images.

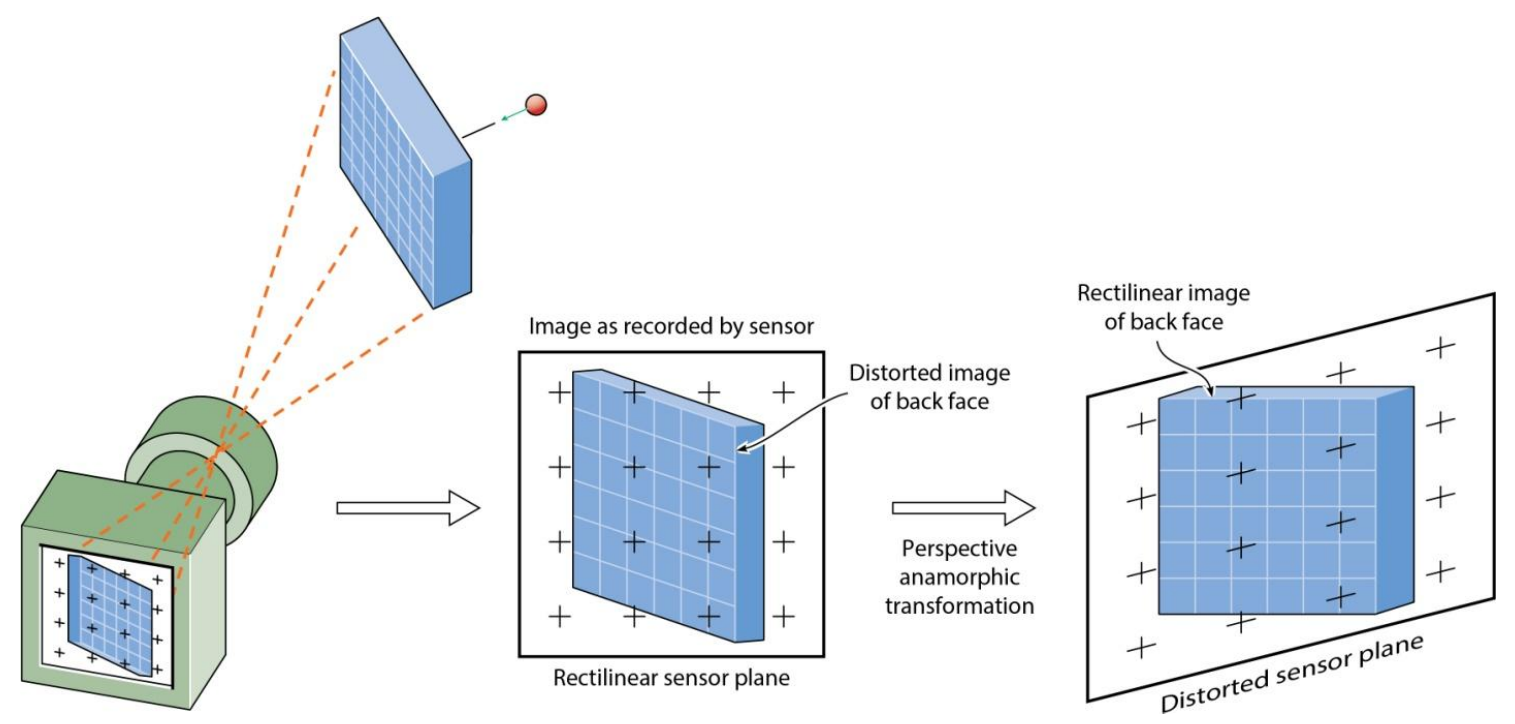

Figure B.1. Sketch showing the oblique visualization of the grid on the Dyneema ${ }^{\circledR}$ encasing and the transformation used to correct for the parallax error. 


\section{Appendix C. X-ray tomogram analysis procedures}

Void segmentation: The intensity value of each voxel within the reconstructed volume scales with the density of the material represented. Therefore, voxels representing air, having a low-intensity, were isolated with Avizo's "threshold" function. The variants of "closing" and "opening" functions refined the selection. The result was output to the software MATLAB, which calculated the total thickness of all voids in the Z-direction as a function of in-plane (X-Y) position and the porosity.

Laminate edge detection: The border of a solid material, i.e. fiber or resin, with air is marked by a high intensity scanning artifact, known as phase contrast. The voxels exhibiting phase contrast, in the regions within one ply thickness of the laminate surface, were selected using the "threshold" function. The variants of "closing" and "opening" functions refined the selection, and the result was output to MATLAB to calculate the thickness of the laminate as a function of in-plane (X-Y) position. 